322
$10-17-\left.7\right|_{\text {Manual }} ^{\text {MA-565 }}$

$\mathcal{O}_{2} \cdot 1000$
UC-15

Reporting Date: June 1974

Issued: September 1974

\title{
Fundamentals of Passive Nondestructive Assay of Fissionable Material
}

\author{
by
}

R. H. Augustson and T. D. Reilly

Nuclear Analysis Research Group, A-1 
Prinled in the Unitod Staten of Amorica. Available from National Technical Inlormation Service

U.S. Department of Commerce

5285 Port hoyal Road

Springfiold, Virginic 2215

Price: Printed Copy 55.45 Microtiche \$1.45

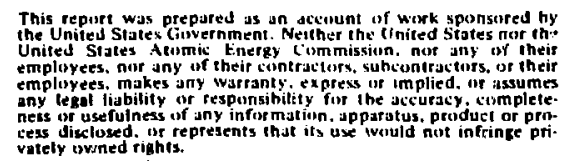




\section{FOREWORD}

The U.S. Atomic Energy Commission's

Nondestructive Assay Training Program in Perspective

Since 1966 the Atomic Energy Commission and the Los Alamos Scientific Laboratory have pioneered the development of new technology for inspection, accountability, and surveillance of special nuclear materials (SNM) to meet foreseen requirements for stringent control of these materials on a time scale consistent with the projected growth of the nuclear in. dustry.

Today's urgent need for tight SNM controls, long foreseen by safeguards people, has recently been underscored by widespread publicity on the problems of nuclear materials theft, diversion, terrorism, etc. The key to an effective balanced national system of stringent safeguards lies in the new nondestructive assay (NDA) techniques and instrumentation being developed and now beginning to be applied throughout the nuclear industry. This now instrumentation, coupled with advances in low"cost computers will provide automated on-line accountability and control of nuclear materials on an essentially real time basis. This automated in-plant control of SNM promises not only stringent, economic safeguards, but also other important economic benefits through upgraded process and quality control, criticality eafoty, and the reduction or elimination of costly shutdown and cleanout procedures at inventory time.

Experience to date in the deveiopment, evaluation, and inplant deployment of NDA equipment has shown that an array of specialized NDA instrumentation is required to measure the many different forms, compositions, and containments in which SlJM appears in the nuclear fuel cycle. The task of successfully implementing and transterring this new technology to the nuclear industry is indeed prodigious.

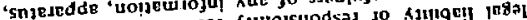

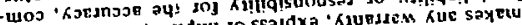

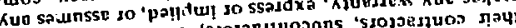

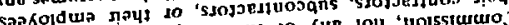

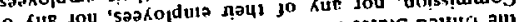

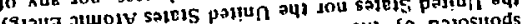
אמנה

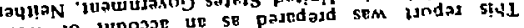


Clecrly the most important single factor i:n the successful trangfer of NDA technology is the training of personnel in practical use of instruments. To that end, the U.S. Atomic Energy Commission establisted the Nondestructiva Assay Training Program at Los Alamos in the fall of 1973. Although enrollment: was initially limited to AEC inspectcrs, future course offerings (on a semiannual basis) wili be available to the growing rumber of users of NDA equipment in both govornment and private sectors of the nuclear community. Courses are designed to cover both the underlying principles and the vise of NDA instruments with emphasis on practical assay and verification measurements of specific interest to attendees. A formal training manual is prepared and used extensively with each course. This first manual provides $\alpha$ general reference on the use of portable NDA instrumentation togethex with a summary of measurement principles basic to the understanding of other more advanced NDA techniques, both passive and active.

The U.S. Atomic Energy Commission Nondestructive Assay Training Program provides an excellent opportunity for direct exchenge of ideas, discussion of special probleris, etc., betiveen the developers and users of NDA instrumentation. Such direst person-to-person interaction has proved by far the most effective method of NDA technology transfer and provides one of the maior practical benefits of the AEC's ongoing NDA training program.

\section{G. Robert Koepin, Group Leader}

Nuclear Analysis Resenrch Group, A-1 


\section{CONTENTS}

Introduction $\ldots \ldots \ldots \ldots \ldots \ldots \ldots \ldots \ldots \ldots \ldots \ldots \ldots \ldots \ldots \ldots$ vii

Abstract $\ldots \ldots \ldots \ldots \ldots \ldots \ldots \ldots \ldots \ldots \ldots \ldots \ldots \ldots \ldots \ldots$

1. Gamma-Ray Production and interaction with Matier $\ldots \ldots \ldots \ldots \ldots \ldots \ldots$

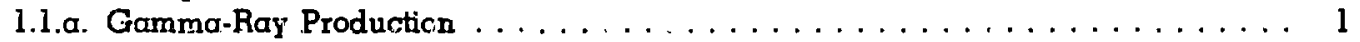

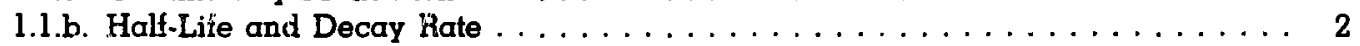

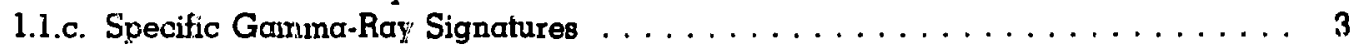

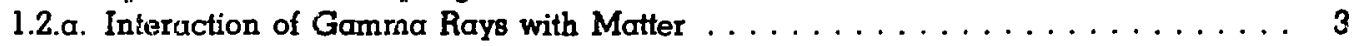

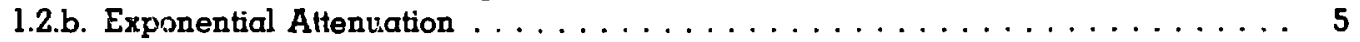

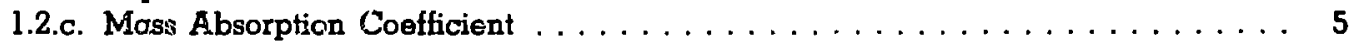

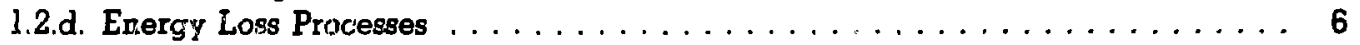

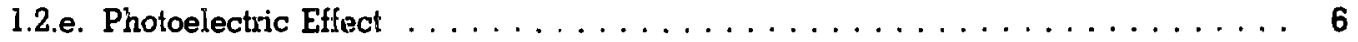

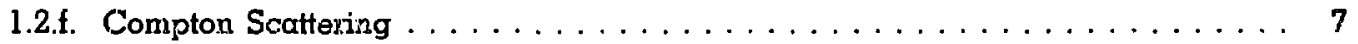

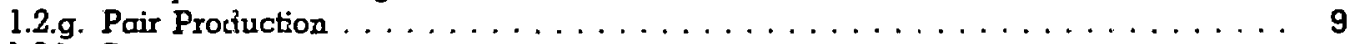

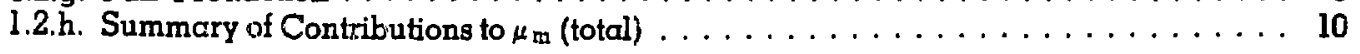

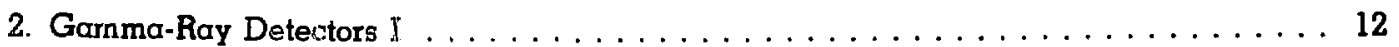

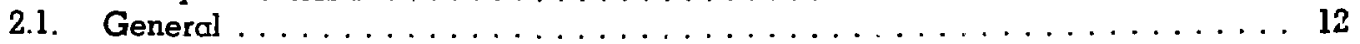

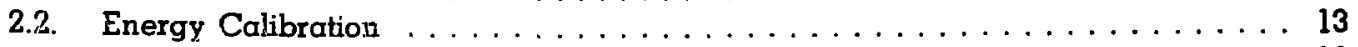

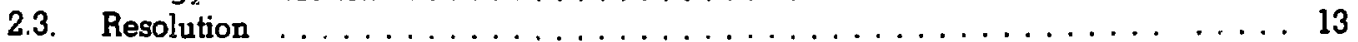

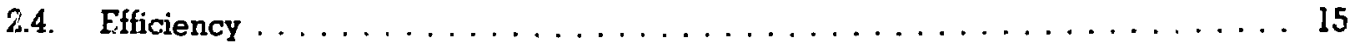

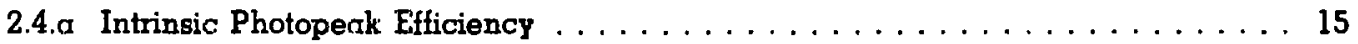

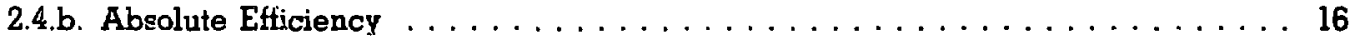

2.4.c. Relative Efficiency . . . . . . . . . . . . . . . . . . . 17

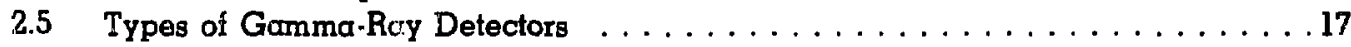

2.5.a. Scintillation Detectors $\ldots \ldots \ldots \ldots \ldots \ldots \ldots \ldots \ldots \ldots \ldots \ldots \ldots$

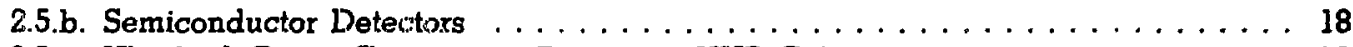

2.5.c. Ultrahigh Purity Germonium Detectors (UHP Ge) . . . . . . . . . . 19

3. Gamma-Ray Detectors II . . . . . . . . . . . . . . . . . . 20

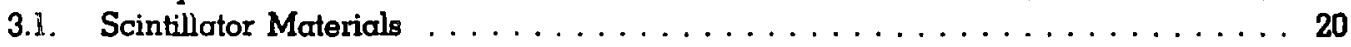

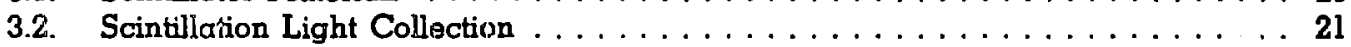

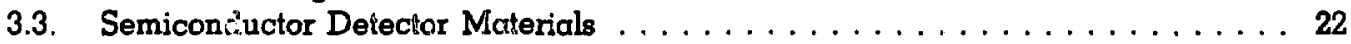

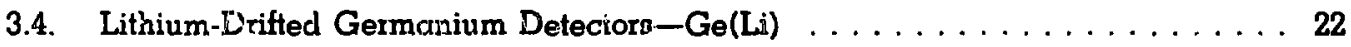

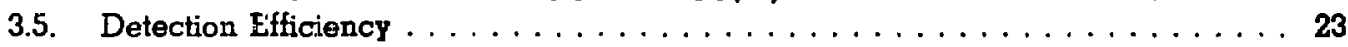

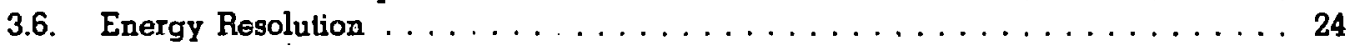

4. Analysis of Gamma-Ray Pulse-Height Spectra $\ldots \ldots \ldots \ldots \ldots \ldots \ldots$

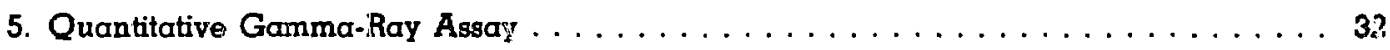

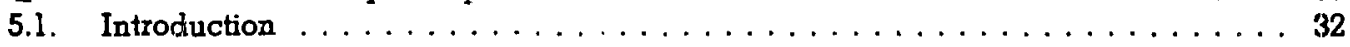

5.2. Gamma-Roy Assay and the Inverse Square Low . . . . . . . . . . . . 33

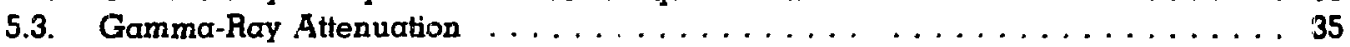

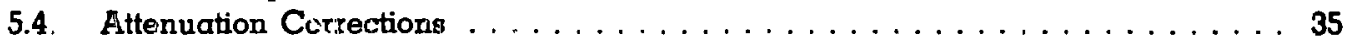

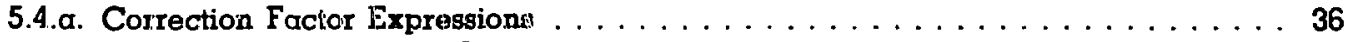

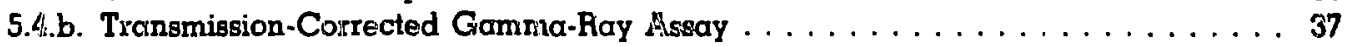

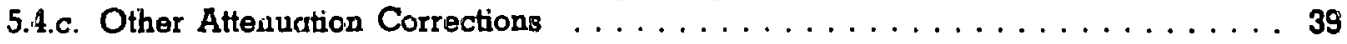


5.5. Example 1: Small Cans of Plutonium Incirerator Ash . . . . . . . . . . . 41

5.6. Example 2: Uranium-Contarninated Air Fïlters . . . . . . . . . . . . . 43

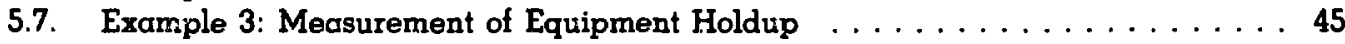

5.8. Instrument Dead Time . . . . . . . . . . . . . . . . . . . 47

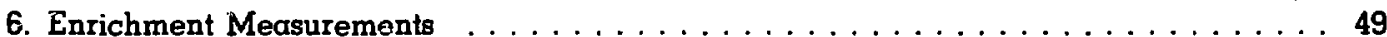

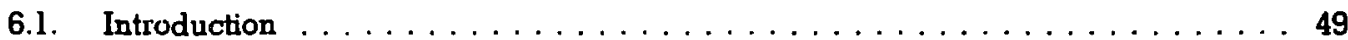

6.2. The Fundamentai Relation . . . . . . . . . . . . . . . . . 49

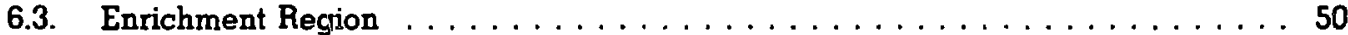

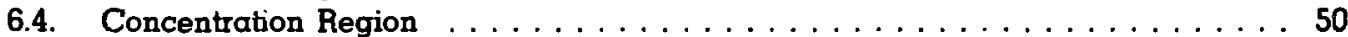

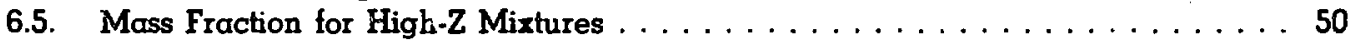

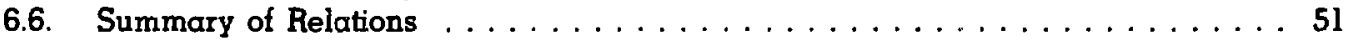

6.7. Example 1 : Measurement of Highly Enriched UF ${ }_{6}$ Cylinders $\ldots \ldots \ldots \ldots \ldots$

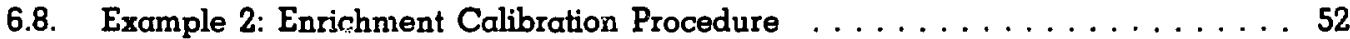

7. Neutron Production and Applicable Signatures $\ldots \ldots \ldots \ldots \ldots \ldots$

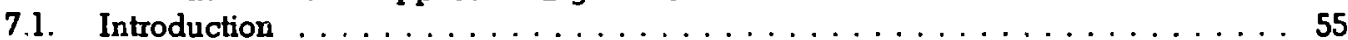

7.2. Alpha-Induced Neitron Emission $\ldots \ldots \ldots \ldots \ldots \ldots \ldots \ldots \ldots \ldots$

7.3. Neutrons from Spoataneous Fission $\ldots \ldots \ldots \ldots \ldots \ldots \ldots \ldots \ldots$

7.4. Interaction of Neutrons with Matter $\ldots \ldots \ldots \ldots \ldots \ldots \ldots \ldots$

7.5.a. Interaction Processes $\ldots \ldots \ldots \ldots \ldots \ldots \ldots \ldots \ldots \ldots \ldots$

7.5.b. Interactions in Which the Neutron Is Absorbed . . . . . . . . . . . . 59

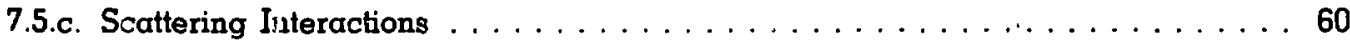

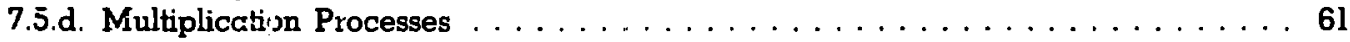

8. Neutron Detectors $\ldots \ldots \ldots \ldots \ldots \ldots \ldots \ldots \ldots \ldots \ldots \ldots \ldots \ldots$

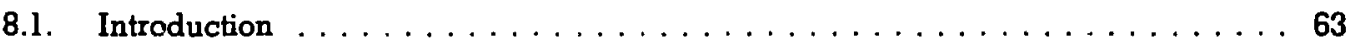

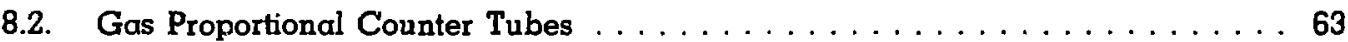

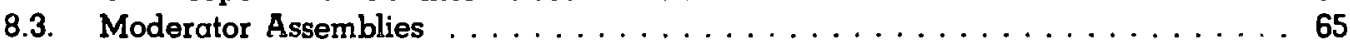

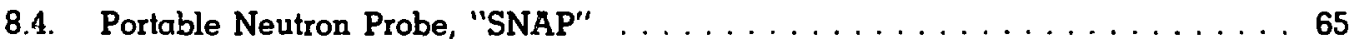

8.5. Other Neutron Detector Types $\ldots \ldots \ldots \ldots \ldots \ldots \ldots \ldots \ldots \ldots \ldots \ldots \ldots$

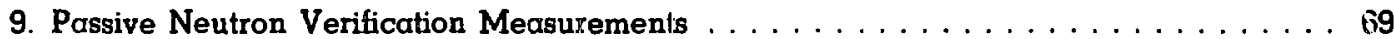

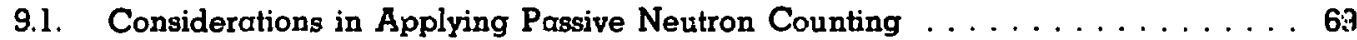

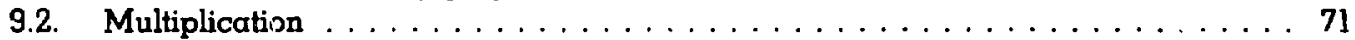

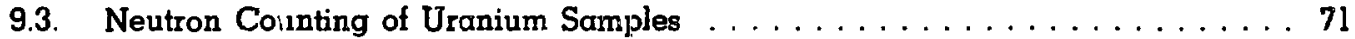

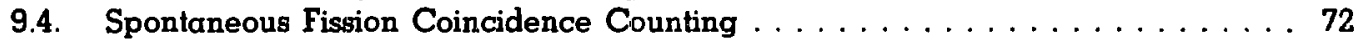

Appendix A: Gamma-Ray Signatures $\ldots \ldots \ldots \ldots \ldots \ldots \ldots \ldots \ldots$

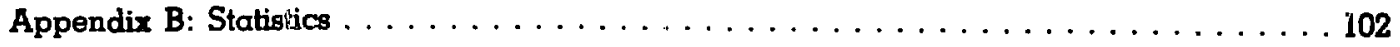

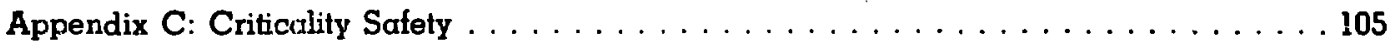




\section{INTRODUCTION}

This manual is written as a general reference to the basic principles of nondestructive assay of fissionable material. It is the foundation for a course on the use of portable instrumentation conducted at the Los Alamos Scientific Laboratory. The manual is written at an introductory level and requires minimal background in rondestructive assay. The philosophy is to explain the basic principles and then use specific examples to illustrate the application of these principles to practical assay problems. It is impossible to discuss all of the mecrsurements required in the nuclear industry; however, by emphasizing the basic concepts the assayist is loetter prepared to handle a wide range of measurement problems. The first six chapters deal with gamma-ray assay including gamma-ray production and interaction, detection principles, quantitative assay, and enrichment measurements. The last three chapters discuss passive neutron measurements including basic signatures and interactions, neutron detectors, and verification measurements. Although the mauual emphasizes portable instrumentation, it includes material on more advanced, nonportable equipment. Lithium-drifted germanium detectors are discussed in detail in Chap. 3. Spontaneous fission neutron detectors are described briefly in Chap. 9. Although this is not directly applicable to portable instrument assay, it provides valuable background material on advanced assay procedures.

Many people have helped to generate this manual and set up the in. itial course session. Larry East was largely responsible for writing Chaps. 3 and 4. Albert Evans, Jack Parker, and Darryl Smith also contributed to the writing. The course instructors, Thomas Atwell, John Foley, Jack Parker, and Roddy Walton, helped decide the content of the manual and were responsible for eetting up the laboratory sessions, which were the bulk of the course. The belp and support of all these people and of Joan Rosul is gratefully acknowledged. 


\title{
FUNDAMENTALS OF PASSIVE NONDESTRUCTIVE ASSAY OF FISSIONABLE MATERIAL \\ by
}

\author{
R. H. Augusteon and T. D. Reilly
}

\begin{abstract}
This manual is an introduction to the fundamental principles of passive gamma-ray and noutron assay for fissionable material accountability.
\end{abstract}

\section{GAMMA-RAY PRODUCTION AND INTERACTION WITH MATTER}

\section{1.a. Gamma-Ray Production}

This section discusses the processes that enable fissionable material to be measured using its naturally occurring camma-ray activity. Gamma rays are a form of high-energy electromagnetic radiation. The brood electromagnetic spectrum shown in Fig. 1.1 ranges from radiowaves to visible light to $x$ rays and gamma rays. Radiowaves and light usually are described in terms of frequency or wavelength, but $x$ rays and gamma rays are characterized by their energy in keV or MeV. Euergy (E) and trequency (v) are directly related by $E=h v$ (Planck's constant, $h$, equals $\left.4.14 \times 10^{-15} \mathrm{eV}-\mathrm{s}\right)$. The relation between energy and wavelength $(\lambda)$ is $E=h c / \lambda$, where $c$ is the speed of light (hc $=1.24 \times 10^{-6} \mathrm{eV}-\mathrm{m}$ ). Gamma rays have very high frequencies and very short

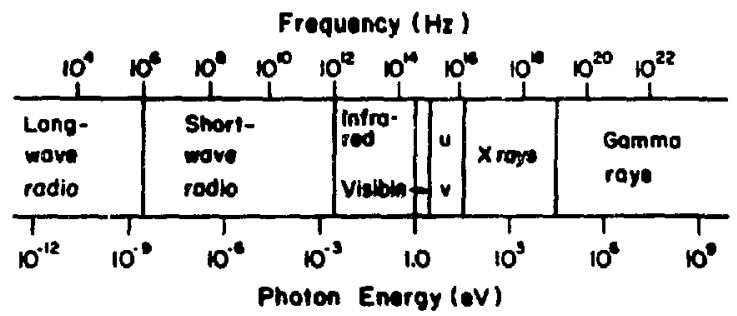

Fig. 1.1 .

The electromagnetic spectrum. wavelengths. Gcmma rays arise from excitations within the nucleus, whereas $x$ rays come from excitations of the atomic electrons.

Many radioactive isotopes emit gamma rays during decay. The energy and intensity of these gamma rays are uniquely characteristic of the isotope. Figure 1.2 shows $a$ portion of the characteristic gamma-ray spectrum of $235 \mathrm{U}$. There is little probability that gamma rays from different isotopes will exactly overlap in energy, therefore, gainina rays can be used to identify uniquely the presence of particular isotopes. All fissionable material is unstable, emitting some form of radiation by alpha, beta, or spon. taneous fission decay. In this decay process, some fiseionable isotopes emit gamma radiation that can be quantitatively and uniquely related to the original isotope. Such a gamma ray can be called a signature of that isotope. For example, ${ }^{235} \mathrm{U}$ alpha decays to ${ }^{231} \mathrm{Th}$, leaving the ${ }^{231} \mathrm{Th}$ in excited states which, in turn, decay and emit gamma rays. The alpha particle is accompanied by a $186-k e V$ gamma ray $54 \%$ of the time. This production rate (186-keV gamma rays/s-g $g^{235} U$ ) is a constant of nature. In an unknown sample, the production rate of $186-\mathrm{keV}$ gamma rays is directly proportional to the number of atoms (or grams) of ${ }^{235} \mathrm{U}$ in that sample. The existence of these unique signatures provides the basis for gammr-rary assay of nuclear material. The measurement of real samples is complicated by many factors; for exam. ple, (1) not all of the gamma rays escape from the 


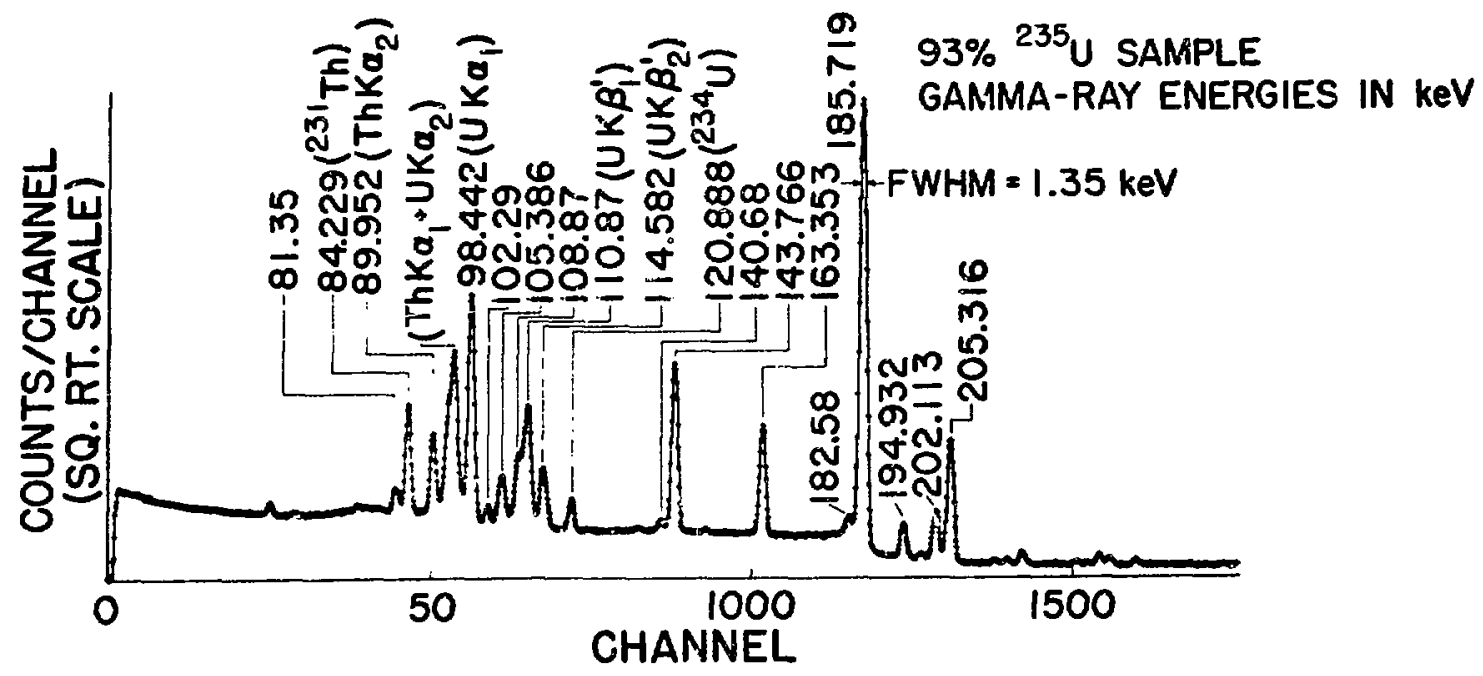

Fig. 1.2.

Characteristic gamma rays of ${ }^{235} U$. This spectrum was measured with a high-resolution Ge(Li) detector.

sample, (2) only a fraction of those that do are detected, and (3) other gamma rays may interfere with the detection of the signature of interest. This is the subject for the first six chapters of this manual.

\section{1.i.b. Half-Life and Decay Rate}

The rate at which an isotope decays is commonly given in terms of its half-life. Half-lifo is defined as the time it takes for one-half of the atoms in a sample to decay. The expression for the number of atoms left in a sample after time $t$ is

$$
N(t)=N(0) e^{-\lambda t}
$$

where

$$
\begin{aligned}
N(0)= & \text { number of atoms at initial time } \\
& 0 . \text { This time is arbitrary; for } \\
& \text { example, it might be the begin- } \\
& \text { ning of sample observation. } \\
N(t)= & \text { number of atoms remaining at } \\
& \text { time } t .
\end{aligned}
$$

where $\lambda$ is given the name "digintegration constant." Half-life and disintegration constant can be used interchangeably, and the relation between them can be derived as follows.

$$
\begin{aligned}
N\left(t_{\frac{1}{2}}\right) & =N(0)=N(0) e^{-\lambda \frac{1}{2}} \frac{1}{2} . \\
e^{\lambda t_{\frac{1}{2}}} & =2, \text { and } \\
t_{\frac{1}{2}} & =\ln 2 / \lambda=0.693 / \lambda .
\end{aligned}
$$

The decay rate, $R$, in disintegrations per second, is given by

$$
R=\frac{d N(t)}{d t}=-\lambda N(t)
$$

$$
=-\lambda\left[N(0) e^{-\lambda t}\right] \text {. }
$$

where the minus sign indicates that the number of atoms is decreasing with time. The decay rate is equal to the product of the disintegration constant and the number of atoms in the sample. The number of atoms in $\mathrm{l} \mathrm{g}$ of a sample with atomic weight $A$ is equal to Avogadro's number $\left(6.023 \times 10^{23}\right)$ divided by the atomic weight. This relation and Eq. (1.2) combine to give a ureful expression for $\mathbf{R}$. 


$$
\begin{aligned}
& N=6.023 \times 10^{23} / \mathrm{A}, \\
& \lambda=0.693 / \mathrm{t}_{\frac{1}{2}} \\
& \mathrm{lyr}=3.156 \times 10^{7} \mathrm{~s}, \text { and } \\
& \mathrm{R}=\lambda \mathrm{N}=\frac{(0.693)\left(6.023 \times 10^{23}\right)}{\mathrm{A} \mathrm{t}_{\frac{7}{2}}\left(3.156 \times 10^{7}\right)} \\
& \mathrm{R}=\frac{1.32 \times 10^{16}}{\mathrm{At}},
\end{aligned}
$$

Where $\mathrm{A}=$ atomic weight.

$$
\begin{aligned}
& t_{1 / 2}=\text { half-life in years, and } \\
& R=\text { decay rate in disintegrations per gram per second. }
\end{aligned}
$$

For example, ${ }^{235} \mathrm{U}$ with $\alpha$ half-life of $7.13 \times 10^{8} \mathrm{yr}$ has a decay rate of

$$
R \frac{\left(1.32 \times 10^{16}\right)}{(235)\left(7.13 \times 10^{8}\right)} \quad 7.88 \times 10^{4} / \mathrm{g}-\mathrm{s} .
$$

Approximately $54 \%$ of the ${ }^{235} \mathrm{U}$ decays lead to a 186 $\mathrm{keV}$ gamma ray, therefore the specific activity of this signature is $\left(0.54 \times 7.88 \times 10^{4}\right)=4.3 \times 10^{4} / \mathrm{g}-\mathrm{s}$. The count rate in a detector would be equal to the product of the specific activity and the overall counting efficiency. If the sample had no self-absorption and were positioned $10 \mathrm{~cm}$ from a $5 . \mathrm{cm}$-diam by $1.3-$ $\mathrm{cm}$-thick NaI detector, the cbserved count rate would be approximately 500 counts $/ 3-8$. Detector efficiency will be discussed in later sections.

\section{1.c. Specific Garnma-Ray Signaturss}

The major gamma-ray signatures for the fissionable isotopes are listed in Table 1.1 . References 1.1 - 1.4 at the end of this chapter contain more complete tabulations of gamma-ray data. As indicated in Table 1.1, some signatures involve gamma rays from a daughter product of the fissionable isolope of interest. Such signatures are usually useful only when the daughter product and parent isotope are in equilibrium. If the rnaterial has recently undergone a physical or chemical process that can separate the isotopes, quantitative measurements are usually reliable cnly after equilibrium has besen reestablished. The time to equilibrium is determined by the longest haif-life in the decay chain between the parent and the duughter (this includes the balflife of the daughter). If four half-lives have elapsed since the separation time, the concentration of the daughter product will be within $6.25 \%$ of the equilibrium concentration. Consider the following example.

$$
{ }^{241} \mathrm{Pu} \stackrel{a}{\longrightarrow}{ }^{237} \mathrm{U} \frac{\mathrm{\theta}^{-}+208 \mathrm{keV} \gamma}{6.75 \text { days }}{ }^{237} \mathrm{~Np} .
$$

Following a chemical separation of uranium and plutonium, the rate of the 208-keV gamma rays will increase with time as equilibrium is restablished between ${ }^{24} \mathrm{Pu}$ and ${ }^{237} \mathrm{U}$. Within 4 to 5 wh, the 208keV activity will stcbilige and will be proportional to ${ }^{241} \mathrm{Pu}$. Consider another example.

$$
\begin{aligned}
& { }^{238} \mathrm{U} \stackrel{a}{\longrightarrow}{ }^{234} \mathrm{Th} \frac{\beta^{-}}{24.1 \mathrm{~d}} \\
& { }^{234 \mathrm{~m} \mathrm{~Pa}} \frac{\beta^{-}+1001 \mathrm{keV} \gamma}{1.18 \mathrm{~min}}{ }^{234} \mathrm{U} .
\end{aligned}
$$

The thorium and protactinium can be separated from uranium by a veriety of chemical and physic m processes: (1) diffusion of $\mathrm{UF}_{6}$ through a porous barrier, (2) melting or vaporization of solid $\mathrm{UF}_{6}$, and (3) various oxicie ccnversion processes. After such a separation, 3 to 4 months are required before the $1001-\mathrm{keV}$ gamma ray is a reliable signature for ${ }^{238} \mathrm{U}$.

\section{2.a. Interaction of Gamma Rays with Matter}

The interaction of gamma rays with matter influences assay measursments in two ways. First, as the gamma rays travel out of the sample they interact with the material in that sample, including the fissionable material emitting the gamma raye. These intercctions reduce the number of gammas that leave the sample with their original energy. This is called sample attenuation. Second, all nuclear detectors require a moving charged particle to initiate the detection process. To be detected, a gamma ray, which is electricaily neutral, must transfer some of its 
TABLE 1.1

MAJOR GAMMA-RAY SIGNATURES FOR THE FISSIONABLE ISOTOPES

\section{Isotope}

$235 \mathrm{U}$

${ }^{238} \mathrm{U}$

${ }^{238} \mathrm{Pu}$

${ }^{239} \mathrm{Pu}$

239pu

$240 \mathrm{pu}$

${ }^{241} \mathrm{Pu}$

${ }^{241} \mathrm{Am}$

${ }^{242} \mathrm{Pu}$
1001.10

766.40

766.40

152.77

413.69

129.28

$1.4 \times 10^{5}$

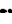

207.98

164.59

148.60

59.54

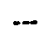
$3.9 \times 10^{1}$

$1.5 \times 10^{5}$

$6.5 \times 10^{6}$

$3.4 \times 10^{4}$ $\frac{\begin{array}{c}\text { Intensity } \\ (\mathrm{g}-\mathrm{s})^{-1}\end{array}}{4.3 \times 10^{4}}$

$1.0 \times 10^{2}$ Plutonium-239 has over 100 gamma rays, some of which are useful for careful work with $\mathrm{Ge}(\mathrm{Li})$.

Several weak gamma rays but all suffer bad interference from gammas of other isolopes. Requires very careful work with high-resolution detectoz to make use of any of them.

Actually from ${ }^{237} \mathrm{~J}$ daughter and requires about 25 days after chemical separation to come into equilibrium at stated value. May also have a few percent interference from ${ }^{241} \mathrm{Am}$ which emits same gamma. Nevertheless a good clean strong gamma useful with both $\mathrm{NaI}$ and $\mathrm{Ge}(\mathrm{Li})$.

Useiul with $\mathrm{Ge}(\mathrm{Li})$. Also from ${ }^{237} \mathrm{U}$.

Useful with $\mathrm{G}_{\Theta}(\mathrm{Li})$. Direct from ${ }^{241} \mathrm{Pu}$.

Very strong gamma but attenuation problems. Useful with $\mathrm{Ge}(\mathrm{Li})$ or $\mathrm{NaI}$. Has several other much less intense garnmas sometimes useful for $\mathrm{Ge}(\mathrm{Li})$ work.

No useful gamma rays at all. Nature failed us at this point. 
energy to electront in the detector. Understanding the interaction proceseses in therefore of fundamental importance to the assayist. For more complete information the book by Evane 1.5 is recommended.

\section{2.b. Exponential Attenuation}

The intencity of $\alpha$ beam of $x$ roys or yamma rays is reduced on paseage through mattor. This attenuation is proportional to the incident intensity and the thickness of the material. Figure 1.3 illustrates the absorption experienced by a beam of gamma rays of initial intensity $\mathrm{I}_{\sigma}$ Equation (1.5) is the fundamental law of gamma-ray attenuation.

$$
I=I_{0} e^{-\mu_{l} L}
$$

The proportionality constant $\mu_{Q}$ is called the linear absorption coefficient. Its magnitude depends on the absorbing material and the gamna-ray energy. Figure 1.4 illustrates the attenuation of gamma rays as they pass througn various thicknesses of lead. Note that $400-k e V$ gamma rays are significantly more penetrating than those at $200 \mathrm{keV}$. Due to the exponential nature of their absorption, gamma rays do not have a definite range as do alpha or beta particles. The quantity $1 / \mu_{Q}$ is often called $\alpha$ mean tree path; it is the thickness of the absorber which reduces the gamma-ray intensity to $1 / e(0.37)$ of its initial value.

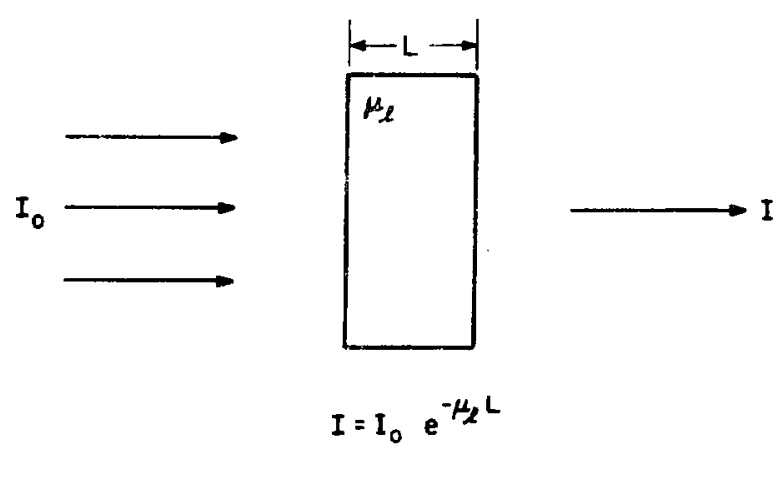

Fig. 1.3.

Fundamental law of gamma-ray attenuation.

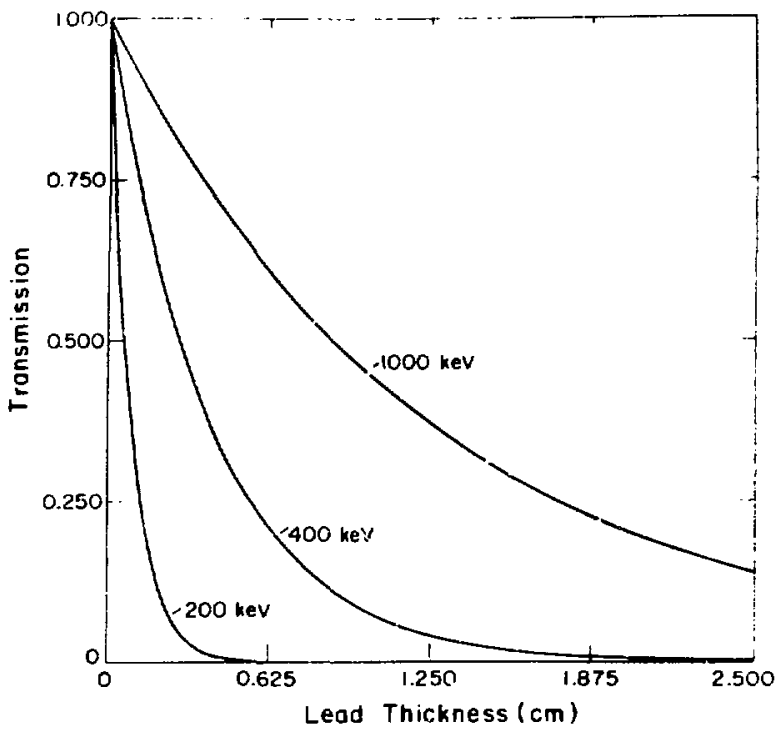

Fig. 1.4.

Exponential attenuation curves for lead.

\section{2.c. Mass Absorption Coofficiont}

In addition to the linear absorption coefficient $\mu_{\Omega}$, it is convenient to define a mass absorption coefficient $\mu_{m}$ where

$$
\mu_{\mathrm{m}}=\frac{\mu_{l}}{\rho}
$$

and $\rho$ is the density of the material. The mass absorption coefficient is more fundamental than the linear absorption coefficient because it is independent of the density and physical state (gas, liquid, or solid) of the absorber. This is important in the nuclear fuel cycle where material can be found in various chemical forms $\left(\mathrm{UF}_{6}, \mathrm{UC}_{\bar{i}}\right.$, etc.), and in combination with other matrix material such as carbon powder or rubber gloves. Tables of absorption coefficients 1.6 will usually list mass absorption coefficients. Equation (1.5) can now be written as

$$
\text { I- } I_{0} e^{-\mu} m^{x} \text {. }
$$

where $x=\rho \mathrm{L}\left(\mathrm{g} / \mathrm{cm}^{2}\right)$. For a sample with more than one element, you can calculate $\mu_{\mathrm{m}}$ total by 


$$
\left(\mu_{\mathrm{m}}\right)_{\text {total }}=\left(\mu_{\mathrm{m}}\right)_{1} \mathrm{w}_{1}+\left(\mu_{\mathrm{m}}\right)_{2} \mathrm{w}_{2}+\ldots .
$$

where w's are the weight tractions of each element in the sample and the $x$ in Eq. (1.7) is equal to the product of sample thickness and bulk density. For example, $\mu_{m}$ for a sample of solid $\mathrm{UF}_{6}, \rho=5.1 \mathrm{~g} / \mathrm{cm}^{3}$ $\left(318 \mathrm{lbo} / \mathrm{ft}^{3}\right)$ at $200 \mathrm{keV}$, is

$$
\begin{aligned}
& \mu_{m}=\left(\mu_{m}\right)_{1} \frac{238}{238+6(19)}+\left(\mu_{m}\right)_{2} \frac{6 \times 19}{238+6(19)}, \\
& \mu_{m}=(1.23)(0.68)+(0.123)(0.32)=0.88 \mathrm{~cm}^{2} / \mathrm{g}, \text { and } \\
& \mu_{l}=\left(0.88 \mathrm{~cm}^{2} / \mathrm{g}\right)\left(5.1 \mathrm{~g} / \mathrm{cm}^{3}\right)=4.5 \mathrm{~cm}^{-1}, \text { where }
\end{aligned}
$$$$
\left(\mu_{\mathrm{m}}\right)_{1}=\begin{aligned}
& \text { mass absorption coefficient for uranium at } \\
& 200 \mathrm{keV}=1.23 \mathrm{~cm}^{2} / \mathrm{g} \text {, and }
\end{aligned}
$$

$$
\left(\mu_{m}\right)_{2} \equiv \begin{aligned}
& \text { mass absorption coefficient for fluorine at } \\
& 200 \mathrm{keV}=0.123 \mathrm{~cm}^{2} / \mathrm{g} .
\end{aligned}
$$

As an additional example, consider a 5-liter scrap conteiner weighing $5 \mathrm{~kg}$. The material in the container consists of the oxides of low-Z elements and $250 \mathrm{~g}$ of uranium spread uniformly throughout the container. Only a small error is made if the matrix material is assumed to attenuate like oxygen. Making this asnumption, $\mu_{m}$ can be calculated as follows.

$$
\begin{aligned}
\mu_{\mathrm{m}}(200 \mathrm{keV}) & =(1.23)\left(\frac{250 \mathrm{~g}}{5000 \mathrm{~g}}\right)+(0.123)\left(\frac{4750 \mathrm{~g}}{5000 \mathrm{~g}}\right), \\
\mu_{\mathrm{m}}(200 \mathrm{keV}) & =\frac{0.061}{U \text { contribution }}+\frac{0.117}{0 \text { contribution }} \\
& =0.178 \mathrm{~cm}^{2} / \mathrm{g}, \\
\rho_{\mathrm{bulk}} & =\frac{5000 \mathrm{~g}}{5000 \mathrm{~cm}^{3}}=1 \mathrm{~g} / \mathrm{cm}^{3}, \text { and } \\
t_{2} & =0.178 \mathrm{~cm}^{2} / \mathrm{g} \mathrm{x} 1 \mathrm{~g} / \mathrm{cm}^{3} \\
& =0.178 \mathrm{~cm}^{-1} .
\end{aligned}
$$

\section{2.d. Enorgy Inese Proconsen}

There are numerous processes by which gamma rays interact with matter and lose energy. These processes contribute in differing degrees for different energy gammas. The gamma rays emitted in nuclear decay usually have energies ranging from $100 \mathrm{keV}$ to $a$ few $\mathrm{MeV}$. In this range the three main processes by which gammas interact with matter are: photoelectric effect, Compton scattering, and pair production. These are schematically represented in Fig. 1.5.

\section{2.e. Photooloctric Effoct}

For $\alpha$ photoelectric interaction the incident gamma ray is totally absorbed by one of the atomic electrons, causing that electron to move through the material with an energy

$$
E_{e}=E_{Y}-B \text {. }
$$

where $E_{\gamma}$ is the energy of the incident $\gamma$, and $B$ is the binding energy of the electron. The energy represented by $B$ appecrs as characteristic $x$ rays of the atom whose electron absorbed the incident $\gamma$. These $x$ rays are in turn absorbed by the material, ejecting further lower energy electrons. The photoslectric process is important for the detection of gammas in that it converts all the gamma-ray energy into the motion of charged partivles. This electron moticn is transformed into an electrical pulse ot the output of the detector. For $\mathrm{Nal}$ and semiconductor detectors, the size of a pulse produced by a photoelectric event is proportional to the energy of the gamma that caused the event.

Table 1.2 illustrates the dependence of the photoelectric effect on atomic number $\mathrm{Z}$ and gamma energy. As the $Z$ of the material increases, $\mu_{m}$ (photoelectric) increases rapidly. Not only does $\mu_{\mathrm{m}}$ (photoelectric) increase, bul also

$$
\frac{\mu_{\mathrm{m}} \text { (photoelectric) }}{\mu_{\mathrm{m}}(\text { total })}
$$

gets larger. At $Z=92$ and $E_{\gamma}=200 \mathrm{keV}$, the dominant interaction process is, by far, the photoelectric effect. For higher energies the effect drops off rapidly as seen when comparing the $\mu_{m}$ 's at $200 \mathrm{keV}$ and $400 \mathrm{keV}$. The $Z$ and $E_{\gamma}$ dependences go roughly as $\mathrm{Z}^{3} / \mathrm{E}_{\gamma}{ }^{3}$. 


\section{Photoelectric Effect}

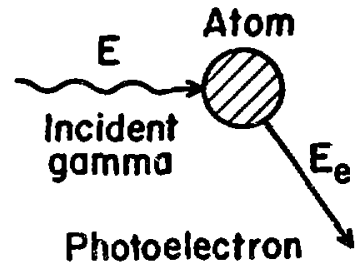

Compton Scottering

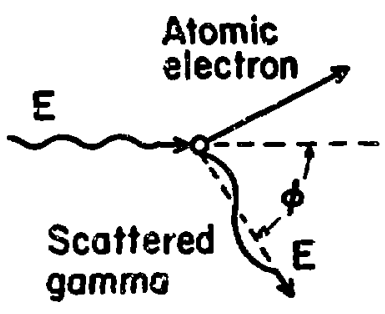

\section{Poir \\ Production}

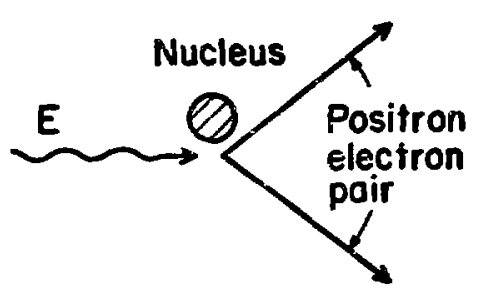

Fig. 1.5.

Representation of najor gamma-ray' interaction processes.

TABLE 1.2

$Z$ DEPENDENCE OF PHOTOELECTRIC MASS ABSORPTION COEFFICIENTS FOR $E_{\gamma}=200$ and $400 \mathrm{keV}$

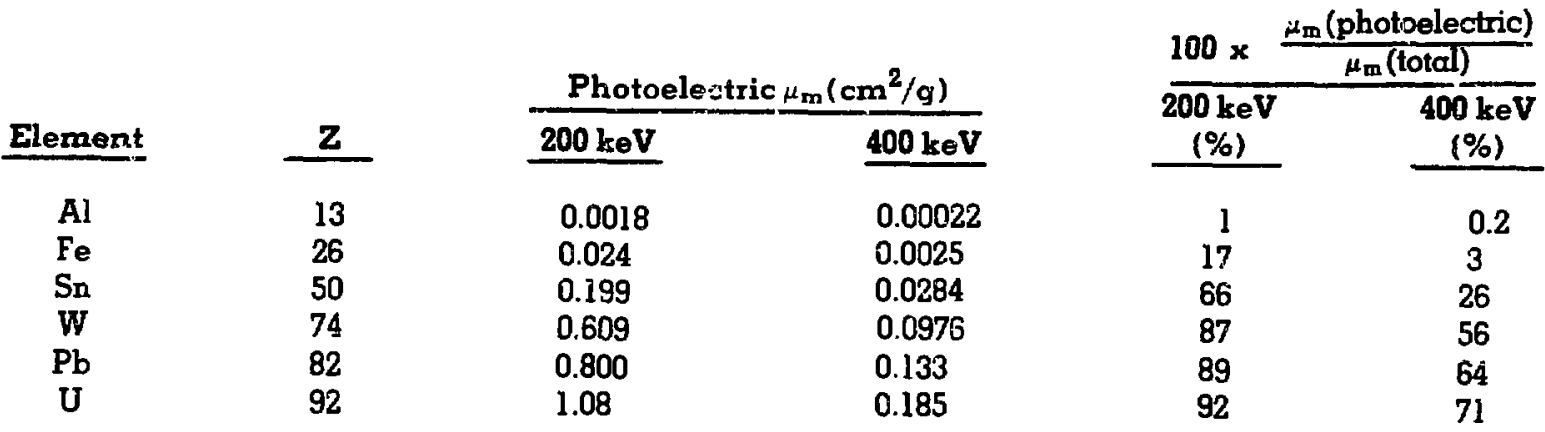

An interesting feature of $\mu_{\mathrm{m}}$ (photoelectric) is the presence of absorption edges. Figure 1.6 siows $\mu_{\mathrm{m}}$ (PE) vs $E_{y}$ for lead. At approximately $90 \mathrm{keV}$ there is a large step increase in $\mu_{\mathrm{m}}(\mathrm{PE})$ labeled the " $\mathrm{K}$ absorption edge." At the $\mathrm{K}$ edge a gamma ray has just enough energy to start removing the most tightly bound $\mathrm{K}$ electrons from the atom. Because there are more possibilities for interaction, $\mu_{\mathrm{m}_{\mathrm{t}}}$ increases rapidly. For larger values of $Z$, the $K$ edge occurs at higher energies. For example, the $\mathrm{K}$ edge of uranium is $116 \mathrm{keV}$. The small steps around $15 \mathrm{keV}$ are called the "L absorption edges." In this region, a gamma ray has just enough energy to remove the next most tightly bound $\mathrm{L}$ electrons from the atom.

\section{2.f. Compton Scattering}

Compton scattering is $\alpha$ process by which the incident gamma ray interacts with a free electron and is scattered with a lower energy, the rest of the energy being taken up by the recoiling electron. Because the outer electrons in an atom are loosely bound and the energies of the incident gamma rays are comparatively high, we can treat these as free electrons. When an incident photon of energy $E_{\gamma}$ strikes a free electron with $\alpha$ rest mass $\mathrm{in}_{3}$, the interaction results in a scattered gamma ray of energy $E_{\gamma}{ }^{\prime}\left(<E_{\gamma}\right)$ and a recoiling electron with energy $E_{0}$, where

$$
E_{e}=E_{Y}-E_{Y}^{*} .
$$




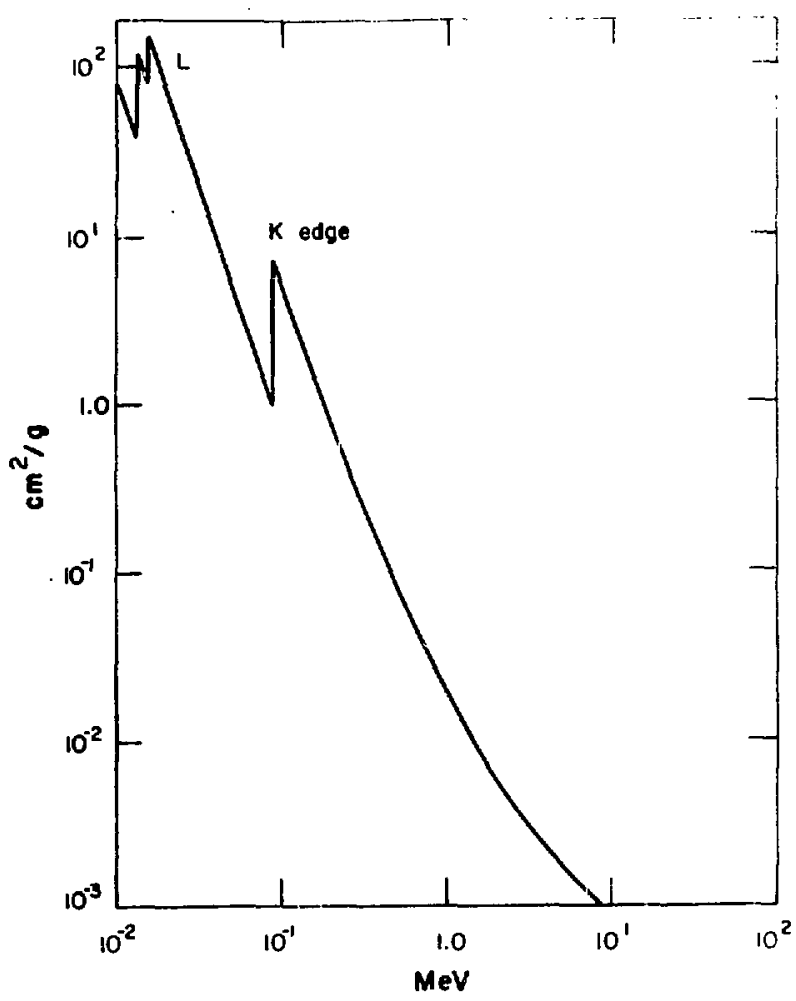

Fig. 1.6.

Photoelectric mass absorption coefficient for iead.

The energy of the scatlered gamma ray is given by Eq. (1.9).

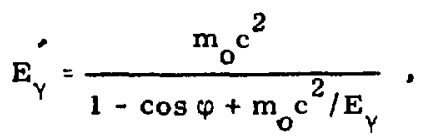

where

$m_{o} c^{2}=$ rest mass of electron $=511 \mathrm{kev}$, and

$\varphi$ - angle between incide:It gamma ray and scattered gamma ray.

$E_{\gamma}$ decreases as the scattering angle increases.

$$
\begin{aligned}
\text { For } \varphi & =0^{\circ}(\cos \varphi=1), \\
E_{Y}^{*} & =E_{Y} .
\end{aligned}
$$

Small-angle scatters result in very little energy loss.

$$
\begin{aligned}
& \text { For } \varphi=180^{\circ}(\cos \varphi=-1), \\
& E_{Y}^{\circ}=\frac{m_{o} c^{2}}{2+m_{0} c^{2} / E_{Y}} \\
& \text { if } E_{Y} \gg m_{o} c^{2}, \\
& E_{Y}^{\circ} \sim m_{0} c^{2} / 2 \sim 256 \mathrm{keV} .
\end{aligned}
$$

The maximum energy transfer occurs when the scattered gamma ray goes in the back direction and the recoil electron goes in the forward direction. These equations show there is a maximum energy that can be given to the electron in a Compton collision. This maximum onergy is given by

$$
E_{\max }=E_{\gamma}\left(I+m_{0} c^{2} / 2 E_{\gamma}\right)^{-1}
$$

For example, if $E_{\gamma}=200 \mathrm{keV}$, then $E_{\max }=88 \mathrm{keV}$, which is the maximum energy that can be deposited in a detector in a single Compton irteraction. Figure 1.7 is a graph of Eq. (1.10). The scattered gamma ray can escape from the detector or can be absorbed in fuxther interactions. Figure 1.8 shows $\propto \mathrm{Ge}(\mathrm{Li})$ detec tor spectrum of ${ }^{137} \mathrm{Cs}_{3}$ a monoenergetic gamma-ray source. The shoulder below the full energy photopeak is caused by single Compton interactions in the detector. The fecture labeled Compton edge represents the maximum energy that can be deposited in the detector in a single Compton scattering.

Table 1.3 illustrates an interesting feature which makes it easier to calculate Compton scattering contributions. Note that the Compton effect has only small dependence on $Z$. Therefore at a given $E_{\gamma}$, for low. $\mathrm{Z}$ material where the photoelectric effect is small. the $\mu_{m}$ (Compton) dominates and is approximately constant. So the assumptions made in the example in Sec. 1.2.c were justified. The energy dependence of 


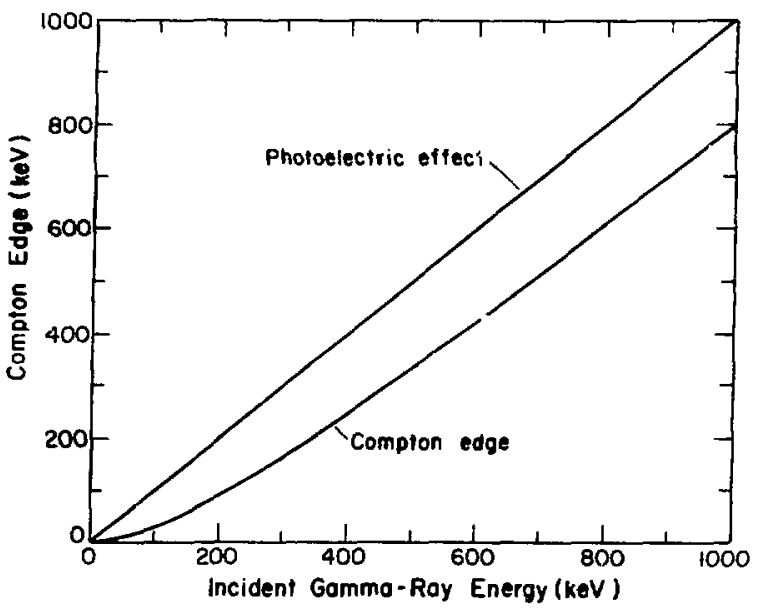

Fig. 1.7 .

Compton edge $\left(E_{\text {max }}\right)$ is incident gamma-ray enterg.:

Compton scattering is much smaller than that of the photoelectric effect. Between 100 and $1000 \mathrm{keV}$, the $\mu_{\mathrm{m}}(\mathrm{PE})$ will decrease by factors of 100 and greater, whereas $\mu_{\mathrm{m}}$ (Compton) will decrease by 2 to 3 .

There are assay situations in which the attenuation effect due to Compton scattering can be overpredicied. Consider the case in which the dominant interaction process in the sample to be measured is Compton scattering, as would be the case for low-Z matrix material (combustible waste) or for higher energy gamma rays such as the $\sim 1-\mathrm{MeV}$ lines used as $238 \mathrm{U}$ signatures. If the detector is large (a $12.7-\mathrm{cm}$-diam $\mathrm{NaI}$ ), and is relatively close to the sample, then some fraction of those gammas scattered through small angles can be counted in the energy window of the data collection electronics. Remember that the gamma-ray's energy loss depends on the angle through which it is scattered. Small-angle scattering corresponds to small energy loss. Therefore even though a garnma was scattered, it can be counted in the full-energy peak as an unattenuated event. This effect causes the observed attenuation to be lower than predicted by $\mu_{\Omega}$ and Eq. (1.5). It is particularly pronounced for low-resolution detectors, such as NaI, which require wide count rindows. For high-resolution detectors, such as $\mathrm{Ge}(\mathrm{Li})$, even a small energy loss reinoves the scattered gamma ray from the full-energy peak. This points up a major advantage of $\mathrm{Ge}(\mathrm{L}$ i): it is easier to interpret the spectral information and estimate sample attenuation.

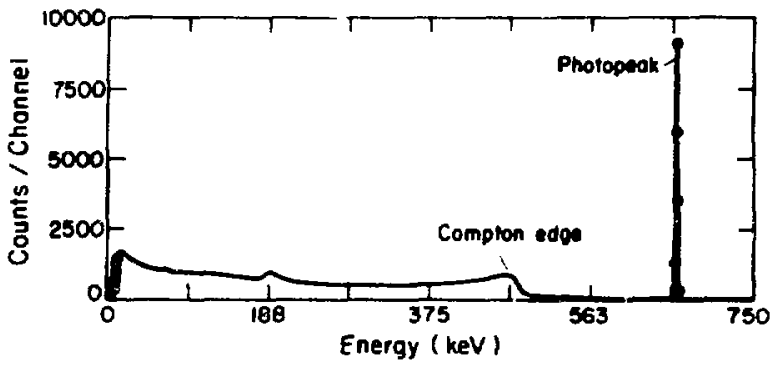

Fig. 1.8.

$\mathrm{Ge}(\mathrm{Li})$ detector spectrum of ${ }^{137} \mathrm{Cs}$ showing full energy photopeak and Compton edge from 662keV gamma ray.

TABLE 1.3

$\mu_{\mathrm{m}}$ FOR COMPTON SCATTERING

\begin{tabular}{|c|c|c|c|}
\hline \multirow[b]{2}{*}{ Eloment } & \multirow[b]{2}{*}{$\mathbf{z}$} & \multicolumn{2}{|c|}{$\mu_{m}$ (Compton) } \\
\hline & & $200 \mathrm{keV}$ & $400 \mathrm{keV}$ \\
\hline $\begin{array}{l}O \\
\mathrm{Al} \\
\mathrm{Fe} \\
\mathrm{Sn} \\
\mathrm{W} \\
\mathrm{Pb} \\
\mathrm{U}\end{array}$ & $\begin{array}{r}8 \\
13 \\
25 \\
50 \\
74 \\
82 \\
92\end{array}$ & $\begin{array}{l}0.122 \\
0.118 \\
0.114 \\
0.103 \\
0.099 \\
0.097 \\
0.095\end{array}$ & $\begin{array}{l}0.095 \\
0.092 \\
0.089 \\
0.080 \\
0.077 \\
0.076 \\
0.074\end{array}$ \\
\hline
\end{tabular}

\section{2.q. Pair Production}

The third process by which photons lose their energy is electron-positron pair production. The threshold energy for this process is $1.02 \mathrm{MeV}$. Because most fissionable material signatures are below this threshold, only a brief description of this process rill be given. If a gamma of energy greater than $1.02 \mathrm{MeV}$ strikes a foil of high-Z material, the gamma can disappear, and an electron-positron pair is formed in its place. The probability of this process increases with increasing gamma energy. The conservation of energy requires

$$
E_{Y}=2 m_{0} c^{2}+E_{+}+E_{-} \cdot
$$

where $E_{\gamma}$ is the energy of the incident photon, $2 m_{\circ} c^{2}$ 
is the energy equivalent to the rest mass of the electron and the positron, and $E_{+}, E_{-}$are the resulting energies of the positron and electron, respectively.

\section{2.h. Sunumary of Contributions to $\mu_{\mathrm{m}}$ (total)}

Figure 1.9 shows the relative coniribution of the three interaction processes to the linear absorption coefficient $\mu_{\mathrm{m}} \rho$ for NaI. The photoelectric effect is the largest contributor at lower energies, falling very rapidly with $E_{\gamma}$. Note the $K$ absorption edge of iodine at $33 \mathrm{keV}$. At about $260 \mathrm{keV}$ the Compton and photoelectric effects cross, with the Compton scattering becoming dominant at higher energies. Pair production is not significant until gamma energies reach several $\mathrm{MeV}$. Figure 1.10 illustrates the $\mathrm{Z}$ dependence of $\mu_{m}$ (total) on the material shown. The differences in $\mu_{m}$ (total) from element to element become greater at lower gamma energies. This is a consequence of the $Z^{3} / E_{\gamma}{ }^{3}$ dependence for the photoelectric effect.

Even though two elements have the same mass absorption coefficients, they may hove very different attenuations. For example, at $E_{\gamma}=400 \mathrm{keV}$ what is the attenuation through $2.54 \mathrm{~cm}$ of iron metal compared to aluminum metal?

$$
\begin{aligned}
\mu_{\mathrm{m}} & =0.092 \mathrm{~cm}^{2} / \mathrm{g} \text { for both aluminum and iron, } \\
{ }^{\rho} \mathrm{Al}^{\mathrm{L}} & =2.70 \mathrm{~g} / \mathrm{cm}^{3} \times 2.54 \mathrm{~cm}=6.86 \mathrm{~g} / \mathrm{cm}^{2}, \\
{ }^{\rho} \mathrm{Fe}^{\mathrm{L}} & =7.86 \mathrm{~g} / \mathrm{cm}^{3} \times 2.54 \mathrm{~cm}=19.96 \mathrm{~g} / \mathrm{cm}^{2} \text {, and } \\
\mathrm{e}^{-\mu \rho L} & =0.53 \text { for Al } \\
& =0.16 \text { for } \mathrm{Fe} .
\end{aligned}
$$

Therefore, the attenuation through $2.54 \mathrm{~cm}$ of iron is much greater than that for a similar thickness of aluminum. The mass absorption coefficient is independent of density, but the attenuation (or conversely the transmission) is not.

\section{REFERENCES}

1.1. C. M. Lederer, J. M. Hollander, I. Perlman, "Table of Isotopes," 6th Ed., (John Wiley and Sons, Inc., NY, 1967). This is a tabulation of gamma rays from all radioactive isotopes. It includes nuclear energy level diagrams, x-rcy information, and much more. It is a very useful reference for gamma-ray measurements.

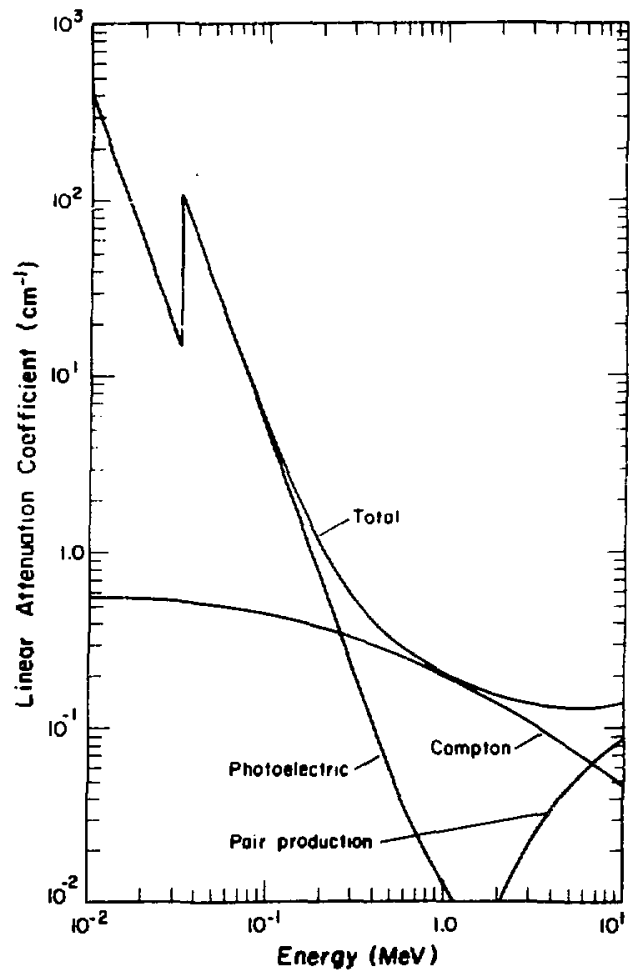

Fig. 1.9.

Linear absorption coefficient for $\mathrm{Nal}$ showing conrributions from photoelectric effect. Compton scattering. and pair production. Note the use of log-log scales. (Data from NBS Circular 583 after "Harshaw Scintillation Phosphors," Harshaw Chemical Company.)

1.2. J. E. Cline, "Gamma Rays Emitted by the Fissionable Nuclides and Associated Isotopes," Idaho Nuclear Corporation report IN-1448 (January 1971) and supplement. "Gamma Rays Emitted by the Fissionable Nuclides and Aseociated Isotopes," Aerojet Nuclear Company report ANCR-1069 (July 1972). These list all gamma rays from the fissionable isotopes and related isotopes. They include typical $\mathrm{Ge}(\mathrm{Li})$ spectra of the materials of interest. There cre some aignificant discrepancies between this reference and Ref. 1.3 regarding line intensities.

1.3. R. Gunnink and R. J. Morrow, "Gamma-Ray Energies and Absolute Branching Intensities for 238, 239. $240,{ }^{241} \mathrm{Pu}$ and ${ }^{241} \mathrm{Am}$," Lawrence Livermore Laboratory report UCRL-51087 (July 1971). This list gamma rays from the plutonium isotopes and show: typical $\mathrm{Ge}(\mathrm{Li})$ spectra. It probably contains the best available data on plutonium gamma-ray intensities. 


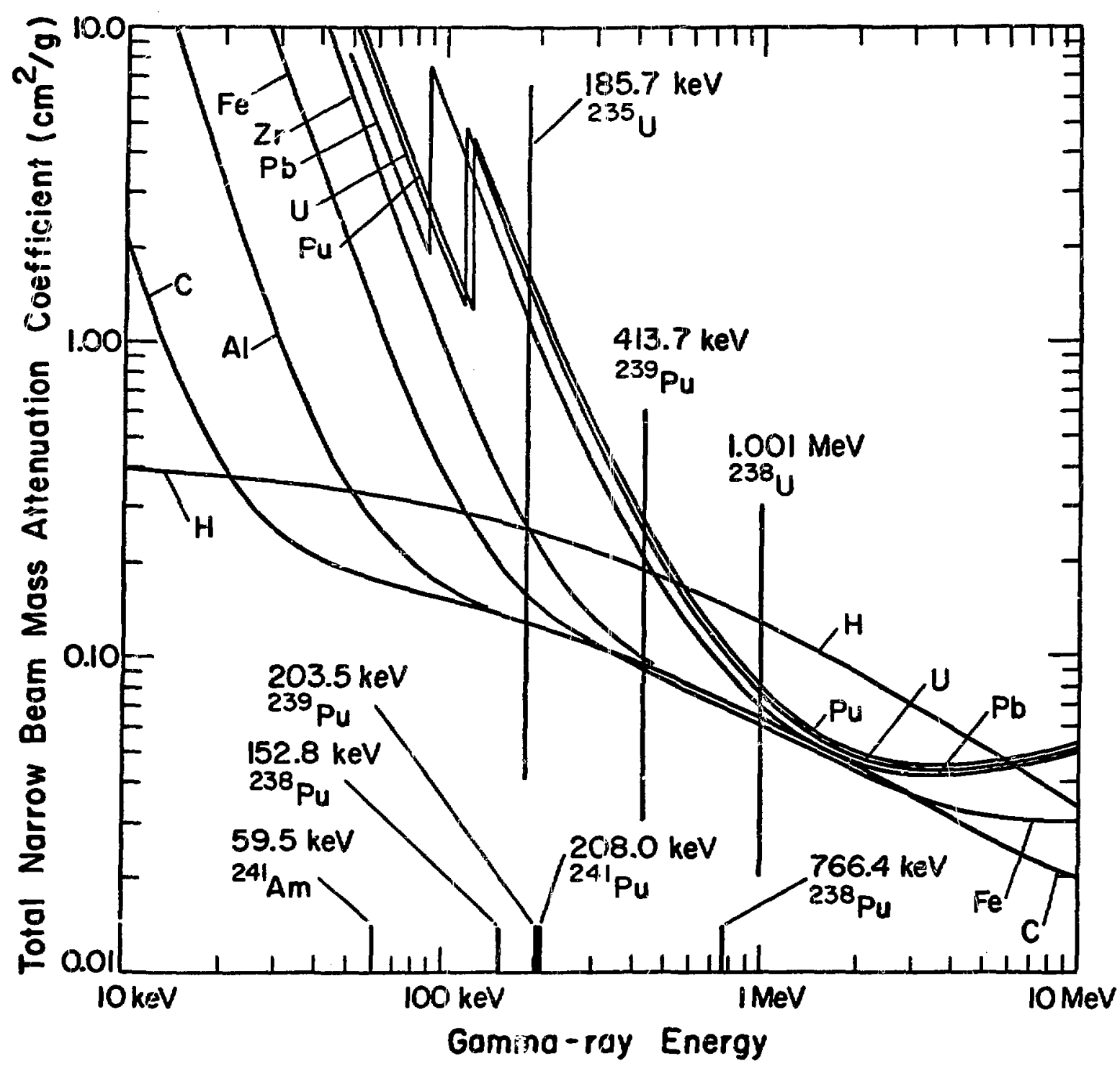

Fig. 1.10 .

Mass absorption coefficients for selected elements.

1.4. R. Gurnink and I. F. Tinney, "Analysis of Fuel Rods by Gamma-Ray Spectroscopy," Lciwrence Livermore Laboratory report UCRL 51086 (Avgust 1971). Appendix C conteins a linting of gamma rays from the fissionable isotopes (similar to Ref. 1.2), which is included in this manual as Appendix A.

1.5. R. D. Evans, The Alomic Nucleus, (McGraw-Hill Book Company, Inc., 1955). Though old, this is one of the best texts on nuclear phyoiz. Recommencind chapters include: 15-Hadioantive Decay, 23-
25-Interaction of Electromagnetic Radiation with Matter, and 26-Statisiical Fluctuations in Nuclear Processes.

1.6. J. H. Hubbell, "Photon Cross Sections, Attenuation Coefficients and Energy Absorption Coefficients from $10 \mathrm{keV}$ to $100 \mathrm{GeV}, "$ NSRDS-NBS-29, Superintendent of Documents, U. S. Government Printing Office, August 1969. This is one of the most recent and most complete listings of gamma-ray attenuation coefficients. 


\section{GAMMA-RAY DETECTOAS I}

\subsection{Goneral}

Nuclear radiation detection is accomplished through the interaction of the radiation with matter. All detection methods generally are kased on the process of excitation or iosization of atome in the detector by energetic charged particles. For gamma radiation, the energetic cliarged particles are electrons produced by one or nore of the three processes discussed in Secs. 1.2.e, 1.2.t, and 1.2.g, namel't photoelectric effect, Compton scattering, and porir production. In gamma-ray spectrometry, only those interactions that result in the total absorption of the gamma-ray photon in the detector are : poitunt. Tharefore, interactions by the Compton eisect alone and the broad energy range of the recoil electrons they produce are a drawback in gamma-ray spectrometry. Consequently, detectors made of high-Z material are preferred, all other factors being equal, to enhance photoelectric (and to a lesser extent pair production) over Compton interactions. Increased detector volume not only increases the detection efficiency due to the higher probability of an interaction occurring within the detector, but also enhances its usefulness in spectrometry because the total absorption of gamma rays becomes more probable due to multiple interactions. After one or more Compton interactions, the scattered gamma ray will have a lower energy thereby increasing the probability of $a$ photoelectric intercction. Through the principle of energy conservation, the same amount of energy will be deposited in the detector regardless of whether total absorption occurred by a single interaction or multiple interactions."

The major features of the detector spectrum are illustrated in Fig. 2.1. The photopeak is formed by those interactions where the entire gamma-ray energy is absorbed in the detector. As explained

\footnotetext{
"Loss of energy due to electrons escaping from the detector volume is ignored. This effect will also be reduced by going to a larger detector volume.
}

above this can be oither a single photoelectric interaction or a multiple interaction involving both the Compton and photoelectric processes. The importance of multiple interactions can be emphasized by noting that, at $662 \mathrm{keV}$, Compton scattering is the dominant interaction process for both $\mathrm{NaI}$ and $\mathrm{Ge}$ (Fig. 1.9). The number of counts under the photopeak is the parameter of interest for nuclear material assay because it is proportional to the mass of the isotope emitting the gamma signature. This parameter is shown as the shaded arec of the $\mathrm{NaI}$ photopeak in Fig. 2.1. The major difference between the two spectra is in the width of the photopeaks. The intrinsic energy widths of the gamma-ray transitions of interest are very small $(0.01-1.0 \mathrm{eV})$. However, the detection process is a statistical one, and the deposition of a fired amount of energy in a detector results in a distribution of electrical pulse heights at the destector output. The peak of this distribution corresponds to the gamma-ray enersy, and the width of the distribution is a property of the particular detectror and its associated electronics. This width is usually charucterized by a parameter called "resolution" and is a function of gamma-ray energy.

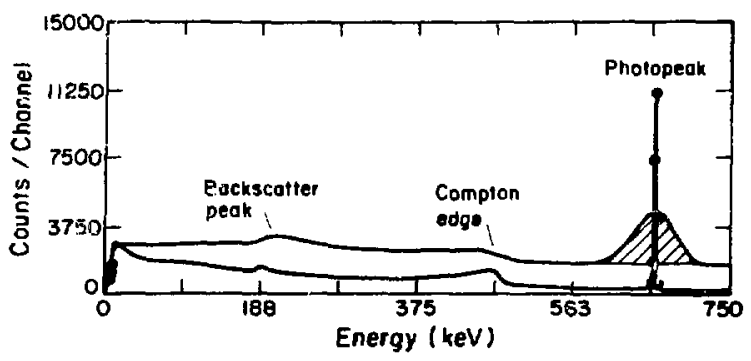

Fig. 2.1.

Nol and Ge( Li) spectra of ${ }^{137} \mathrm{Cr}$ (66) keV). The shaded area represents the net coums in the photopeak and is the parameter of interest for nuclear material assay. The Nal spectrum represents a longer count time: there are approximately (1) limes more counts in the Nal peat than in the Ge(Li). 
The shoulder below the photopeak (beginning at about $475 \mathrm{keV}$ ) is caused by single Compton interactions in the detector. The Compton edge (here at 475 $\mathrm{keV})$ corresponds to the rnaximum energy that can be deposited in a single Compton scattering (Fig. 1.7). The theoretical Complon edge is a sharp cutoff, which is broadened in the measured spectrum because of the finite detector resolution. The region between the photopeak and the Compton edge is filled by events from three major sources: (1) multiple Compton scatters in the detector which deposit less than the initial gamma-ray energy, (2) small-angle Compton scatters in the sample which produce a scattered gamma ray whose ezergy is less than the initial gamma-ray energy, and (3) Compton interactions of higher energy gamma rays (>662 keV) in the detector. The last effect can be due to higher energy gamma rays in the source or in the general background radiation. Common background gamma rays are ${ }^{40 \mathrm{~K}}(1461 \mathrm{keV}),{ }^{200} \mathrm{Tl}(2615 \mathrm{keV}$, a thorium daughtirr), and those from the plutonium and uranium stored around nuclear facilities. These higher energy gamma rays also cause the background continuum above the photopeak. The number of counts in the photopeak is represented by the shaded area in Fig. 2.1. A correction must usually be made to subtract the Compton background continuum from under the photopeak of interest. This is particularly important for the gamma-ray spectra of uranium and plutonium where some of the peaks of interest are always superimposed on very large Compton backgrounds caused by higher energy gamma rays from the sample.

The sroall backscatter peak is caused by a Compton interaction with an electron at the surface of the detector where the recoil elsctron goes out of the crystal, depositing little or no energy, and the scattered gamma ray is absorbed in the detector through other interactions. This is the inverse of the event which leads to the Compton edge, that is, the gamma ray transfers the maximum energy to the electron, but the electron escepes and the backscattered gamma ray is absorbed. The equation for the energy of the backscatter peak is

$$
E_{b s} \cdot \frac{m_{o} c^{2}}{2+m_{o} c^{2} / E_{Y}}
$$

This has a maximum value of $\mathrm{m}_{\circ} \mathrm{c}^{2} / 2=256 \mathrm{keV}$. Because this is basically a surface effect, it is more prominent in small detectors.

\subsection{Energy Calibration}

As described above the pulse-beight spectrum from a NaI or $\mathrm{Cra}(\mathrm{Li})$ detector is usually a series of photopeaks superinposed on $n$ more or less smoothly varying background. The peak locations indicate gamma-ray enercry. To determine the energy, the detector system must firat be calibrated. For $\mathrm{NaI}$ and $\mathrm{Ge}(\mathrm{Li})$ detectors, the relation between gamma energy and output pulse height is nearly linear. Therefore, two known peaks of eufficiently different energy will serve to establish the energy calibration. Let $E_{1}$ and $E_{2}$ be the known energies of the peaks, and let $X_{1}$ and $X_{2}$ be the peal locations as measured in the pulse-height apectrum. The pulse height (X) can be a channel number from a multichannel andyzer spectrum, or it can be a threshold setting for a single-channel analyzer spectrum. The energy $(E)$ associated with a pulse height $(X)$ is then given by

$$
\left.\begin{array}{l}
E=m x+b \\
m=\left(E_{2}-E_{1} / /\left(x_{2}-x_{1}\right)\right. \\
b=E_{1}-m x_{1}
\end{array}\right\} .
$$

Figure 2.2 shows a $\mathrm{Nal}$ spestrum containing the 662$\mathrm{keV}$ peak from ${ }^{137} \mathrm{Cs}$ and the $122-\mathrm{keV}$ peak from ${ }^{57} \mathrm{Co}$. This spectrum can be used to illustrate a sample energy calibration. This procedure can be used for $\mathrm{NaI}$ or $\mathrm{Ge}\left(\mathrm{Li}_{\mathrm{i}}\right)$, multichannel or single-channel electronics. Table 2.1 gives a short list of useful calibration sources and their energies.

\subsection{Resolution}

As mentioned above, the detector resolution characterizes the width of gamma-ray peaks in a measured spectrum. It is usually specitied as the full width at half maximum (FWHM) for a particular gamma-ruy peak. By convention, $\mathrm{Ge}(\mathrm{Li})$ detectors are usually characterized by the FWHM in $\mathrm{keV}$ at the $1332-\mathrm{keV}$ line of ${ }^{60} \mathrm{Co}$. Sodium indide detectors are characterized by the relative width, in percent, of the $662-\mathrm{keV}$ line of ${ }^{137} \mathrm{Cs}$ (i.e., resolution $=100$ - (FWHM in $\mathrm{keV}$ )/662). Figure 2.3 shows an expanded view of the ${ }^{137} \mathrm{Cs}$ peak from Fig. 2.2 and illustrates a sample computation of $\mathrm{NaI}$ rosolution. The indicated resolution of $662 \mathrm{keV}$ is upproximately $12 \%$; the corresponding resolution at $122 \mathrm{keV}$ is approximately $16 \%$. 


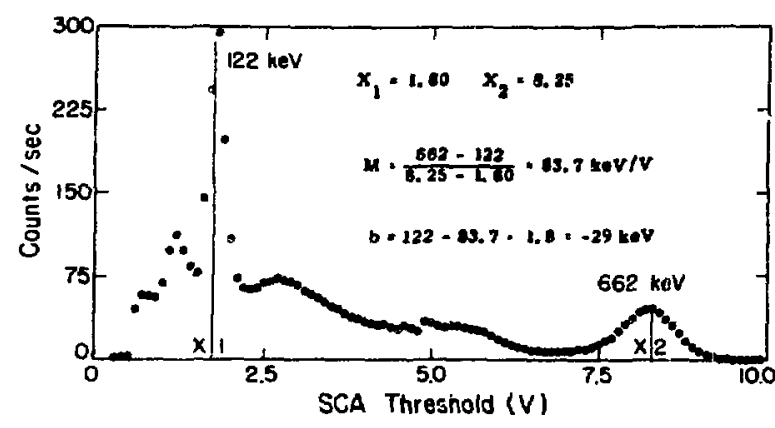

Fig. 2.2 .

Lirersw calibration of a $\mathrm{Nal}$ detector sistem using ${ }^{57} \mathrm{Col}(122 \mathrm{keV})$ and ${ }^{137} \mathrm{Cs}(662 \mathrm{keV})$. The system is a $5.1-\mathrm{cm}$-diam by $1.3-\mathrm{cm}-\mathrm{thick} \mathrm{Nal}$ and an Eherline S.AM-II. The spectrum was acquired with the single-channel aralyzer in the SAM-II by selling a marrow windon and laking short coums at different threshold settings.

This crystal ( $5-\mathrm{cm}$-diam by $1.3-\mathrm{cm}$-thick) is chosen to optimize performance at $185 \mathrm{keV}\left({ }^{235} \mathrm{U}\right)$, and its resolution at $662 \mathrm{keV}$ is only fair. A thicker crystal (5$7 \mathrm{~cm}$ ) should be used for higher energy gamina rays $(5 . \mathrm{cm}$-diam by $5 . \mathrm{cm}$-thick is about right for portable plutonium analysis). A good quality 7.6-cm-diam by $7.6-\mathrm{cm}$-thick detector will have a resolution of about $7 \%$ at $662 \mathrm{keV}$. Figure 2.4 shows the variation of resolution with energy for three different $\mathrm{NaI}$ detectors.

Resolution is a measure of a detector's ability to separate two gamma rays of similar energy. Two

TABLE 2.1

\section{GAMMA-RAY ENERGY CALIBRATION SOURCES}

\begin{tabular}{|c|c|}
\hline Source & $\begin{array}{c}\text { Enexgy } \\
(\mathrm{keV})\end{array}$ \\
\hline${ }^{241} \mathrm{Am}$ & 59.5 \\
\hline${ }^{57} \mathrm{Co}$ & 122.0 \\
\hline${ }^{235} \mathrm{U}$ & 185.7 \\
\hline${ }^{203} \mathrm{Hg}$ & 279.2 \\
\hline${ }^{22} \mathrm{Na}$ & $\begin{array}{r}511.0 \\
1274.5\end{array}$ \\
\hline${ }^{137} \mathrm{Cs}$ & 661.6 \\
\hline${ }^{54} \mathrm{Mn}$ & 834.9 \\
\hline${ }^{88} \mathrm{Y}$ & $\begin{array}{r}898.0 \\
1836.1\end{array}$ \\
\hline${ }^{60} \mathrm{Co}$ & $\begin{array}{l}1173.2 \\
1332.5\end{array}$ \\
\hline
\end{tabular}

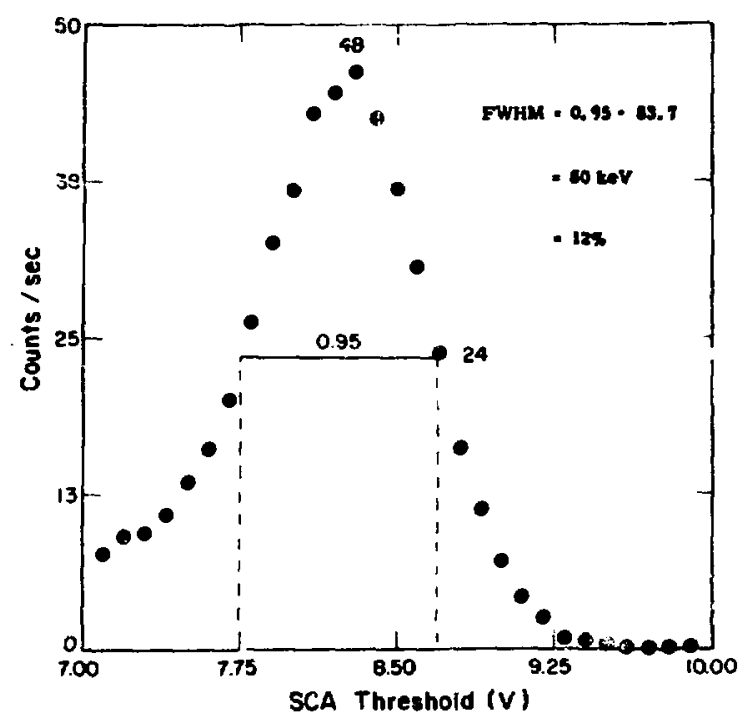

Fig. 2.3.

Expanded view of ${ }^{137} \mathrm{Cs}$ peak from Fig. 2.2 showing colculation of resolution for a $5.1-\mathrm{cm}$ by 1.3-cm-thick Nal deterior.

gamma rays separated by less than two resolution widths (FWHM) are usually not resolved. Under similar counting situations, a high-resolution detector will also have a better signal-to-background ratio.

The resolution of $\mathrm{Ge}(\mathrm{Li})$ detectors will be discussed in detail in Secs. 2.5 and 3.6. The following is a bries

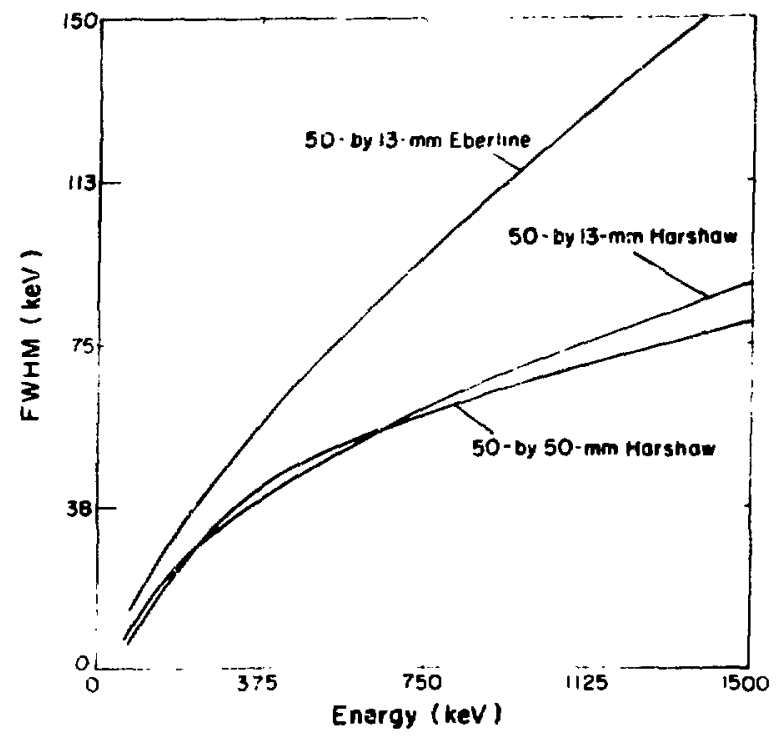

Fig. 2.4.

Rexolution is encergy for fliree Nal detecrors. Same electromics (S.AM-II) used wilh each detcistor. 
description of a convenient proceduse for measuring the resolution of $L \cdot G e(L i)$ spectrometer system af $1332 \mathrm{keV}$. Once the system is operating properly, collect a pectrum from ${ }^{60} \mathrm{Co}$. Adjurt the multichannel analyzer conversion gain and digital offset so that about 800 channels separate the 1173 . and 1332-keV lines from ${ }^{60} \mathrm{Co}$. Now measure the positions of tha two peaks $\left(X_{1}, X_{2}\right)$ and the number of channels, FWHM $=\mathrm{N}_{\mathrm{r}}$, in the 1332-keV peok. The resolution (in keV) of the detector system is then $\mathbf{N}_{I}$. $159 /\left(X_{2}-X_{1}\right)$. This procedure is illustroted in Fig. 2.5.

\subsection{Efficiency}

The efficiency of a counting system depends on a number of factors such as detector size and type and sample-to-detector distance. Several types of efsiency are commonly detined and care must be exercised to determine which type is being used in cay specific instance. Three types of efficiency will be discussed here; the reader should soncentrate on the definitions and not on the names because the names may vary from one writer to another.

\section{4.a. Intrinsic Fhotopeak Efiicioncy}

The intriasic photopeak efficiency is the probability that a gamma ray which strikes the detector will produce a count in the corresponding photopeak, that is, the probability that it will deposit its total energy in the detector. Photopeak efficiency depends on the size, shape, and material of the detector in a complicated way. In general, it increases with detecior size and decreases with gamma-ray energy. Figure 2.6 shows the variation of photopeak efficiency with energy for three different Nal detectors. A very rough estimate of photopeak

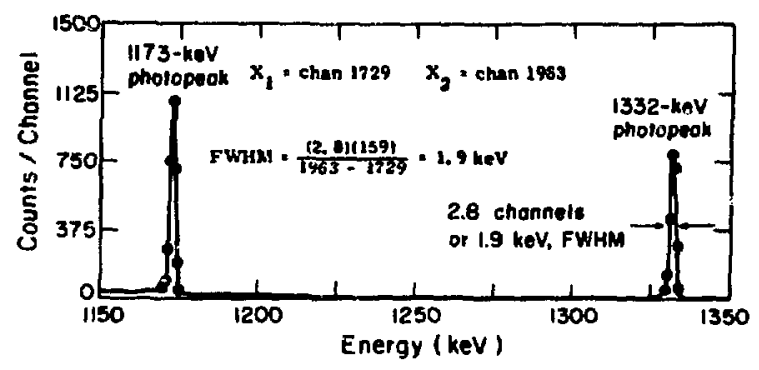

Fig. 2.5.

Measureme'nt of $\mathrm{Ge}(\mathrm{Li}$ detector sustem resolution using " ${ }^{\prime \prime}$ Co.

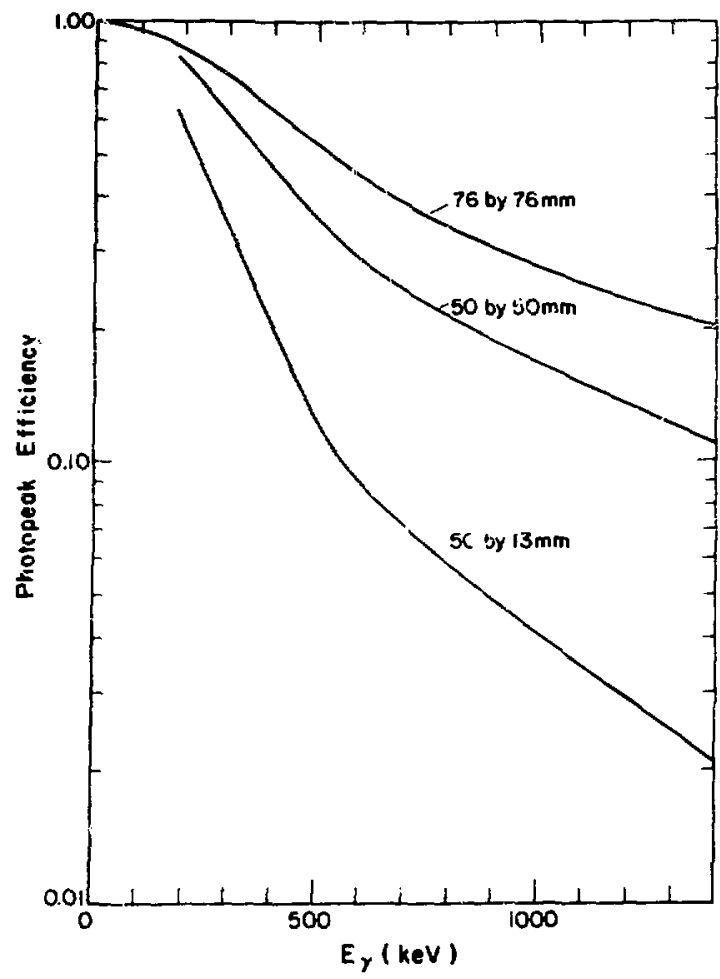

Fig. 2.6.

Photopeak efficiency is energy for three different size Nal detectors.

efficiency can be obtained by using the following expression.

$$
\begin{aligned}
& \varepsilon_{p}-1-e^{-\mu_{l} t} \\
& \varepsilon_{p}-\text { photopeak efficiency } \\
& \mu_{\ell}=\text { linear attenuation coefficient of } \\
& \text { detector at energy of interest, and } \\
& t=\text { thickness of detector. }
\end{aligned}
$$

This expression gives the fraction of incident gamma rays that interact in the detector. It will always give a number larger than the actual photopeak efficiency because all interactions do not result in full-energy deposition. When the photoelectric effect is a lurge part of $\mu_{\mathrm{Q}}$ (e.g., NaI at $186 \mathrm{keV}$ ). Eq. (2.3) is reasonably accurate. It demonstrates the 
dependenc of $\epsilon_{p}$. on gamma-ray energy and detector size. The photopeak efficiency of $\mathrm{Ge}(\mathrm{Li})$ detectors is aiso discussed in Sec. 3.5.

\section{4.b. Absolute Efficiency}

The absolute (or total) efficiency $\epsilon \mathrm{T}$ of a counting system is the probability that a gamma ray emitted from a point source at a particular source-to-detector distance will produce a count in the corresponding photopeak [Eq. (2.4)].

$$
\epsilon_{T}=\frac{\text { phctopeak count rate }}{\text { Y-ray emission rate }} .
$$

The absolute efficiency is the product of the probability that a gamma ray will strike the detector, and the probability that it will interact and produce an event in the photopeak. In general, it depends on the source-to-detector distance, the arad of the detector visible to the source, and the intrinsic photopeak efficiency of the detector. Equation (2.4) ccn be restated as

$$
\begin{aligned}
\epsilon_{\mathrm{T}}= & \epsilon_{\mathrm{g}} \cdot \varepsilon_{\mathrm{p}} \\
\epsilon_{\mathrm{g}}= & \begin{array}{l}
\text { probability of gamma ray striking } \\
\text { detector, sometimes called the } \\
\text { geometric efficiency. }
\end{array}
\end{aligned}
$$

\section{The following is a discussion of $\epsilon_{g}$.}

Consider a point gamma-ray source of intensity ! $(\gamma / \mathrm{s})$ at the center of a sphere of radius $\mathrm{r}(\mathrm{cm})$. The number of gamma rays per second that pass through a unit area of the sphere is called the flux, F. Flux has units of gamma rays per unit area per unit time $\left(\mathrm{r} / \mathrm{cm}^{2}-\mathrm{s}\right)$. Because the surface area of the sphere is $4 \pi \mathrm{r}^{2}, \mathrm{~F}$ can be expressed as

$$
F=\mathbf{r} / 4 \pi \mathbf{r}^{2}
$$

which says that the flux is proportional to $1 / \mathrm{r}^{2}$. The Bux at a distance $2 r$ from the source is one-fourth the flux at $r$ at $3 r$ it is one-ninth the flux at $r$. This is called the inverse square law and il applies to all elec. tromagnetic radiation, including gamma radiation.

Consider a detec lor area $A$ positioned a distance $r$ from a point gamma-ray source. The artifice of a point source (and sometimes a point detector) is conveniently used so that the source-to-detector distance can be specified as a single number. Real sources and detectors have finite extent, and in an exact treatment require an integration of these simple pressions over the extent of the source and detectcr. If the largest dimensions of the source and the detector are much smaller than the average source-todetector disiance, the expressions given here are very good approximations. If $r$ is several times the detector diameter, the fraction of gamma rays striking the detector $\left(\epsilon_{g}\right)$ is given by

$$
\begin{aligned}
\varepsilon_{g}= & \frac{A}{4 \pi r^{2}}, \text { where } \\
A= & \text { area of detector visible at the point } \\
& \text { source. This will be different from } \\
& \text { the frontal area of the detector if } \\
& \text { the detector is collimated or if the } \\
& \text { point source is located off the axis } \\
& \text { of the detector. }
\end{aligned}
$$

From Eq. (2.5), the absolute counting efficiency can now be expressed as

$$
\begin{aligned}
& \varepsilon_{T} \frac{A \cdot \varepsilon_{p}}{4 \pi r^{2}} \text {, where } \\
& \varepsilon_{T}=\text { absolute (or total) efficiency, } \\
& \varepsilon_{p}=\text { photopeak efficiency, } \\
& A=\text { visible area of detector, and } \\
& r=\text { source-to-detector distance. }
\end{aligned}
$$

The absolute efficiency is a function of counting geometry and intrinsic detector efficiency. The absolute efficiency determines the actual measured count rate for a given situation. The measured count rate $(C R)$ is given by

$$
\begin{aligned}
& C R \quad R \cdot \epsilon_{T} \frac{R \cdot A \cdot \epsilon_{p}}{4 \pi r^{2}} ; \\
& R=\text { emission rate of source. }
\end{aligned}
$$


Consider the example given in Se : 1.1.b ${ }^{235} \mathrm{U}$ positioned $10 \mathrm{~cm}$ from a $5-\mathrm{cm}$-diam by $1.3-\mathrm{cm}$-thick Nal crystal).

$$
\begin{aligned}
& R=4.3 \times 10^{4} \mathrm{\gamma} / \mathrm{g}-\mathrm{s} \\
& A=\pi(2.5)^{2}=19.6 \mathrm{~cm}^{2} . \\
& r=10 \mathrm{~cm} .
\end{aligned}
$$

The phoiopeak efficiency $\epsilon_{p}$ can be taken from Fig. $2.6\left(\epsilon_{p} \approx 0.7\right)$, or it can be computed from Eq. (2.3).

$$
\begin{aligned}
& \mu_{l}(186 \mathrm{keV})=1.3 \mathrm{~cm}^{-1}, \\
& t=1.3 \mathrm{~cm}, \\
& \varepsilon_{p}=1-\mathrm{e}^{-1.3 \times 1.3}=0.32 .
\end{aligned}
$$

Substituting these values into Eq. (2.9).

$$
\begin{aligned}
& C R \approx \frac{\left(4.3 \times 10^{4}\right)(19.6)(0.82)}{4 \pi(10)^{2}} \text { and } \\
& C R \approx 550 \text { counts } / g-s .
\end{aligned}
$$

The $(\approx)$ is used because of the many approximations in this discussion. (1) Equation (2.3) for $\epsilon_{p}$ is only a rough approximation. (2) A real sample will not be a point source and the detector diameter here is a significant fraction of the source-to-detector distance (r) so that Eq. (2.8) is only an approximation (reasonably good here). (3) No account has been taken of sample self-attenuation or gamma-ray attenuation in the can surrounding the detector. These considerations would lead to a count rate slightly $k$.ver than computed above. However, if applied with some understanding, these arguments and the associated expressions can yield useful estimates of expected count rate, often within $20 \%$ of the measured rate. For the more complex sample geometries usually oncountered in nuclear material assay, Eq. (2.8) can, in principle, be integrated over the actual sample and detector to yield the absolute efficiency. This procedure is complicated and is sub. ject to considerable error. For accurate work, it should not be used as a substitute for calibzation with known standards. For measurements of odd geometries where a standard is not available for even feasible), such as many process equipment holdup measurements, this $t_{2}$,e of computation may be the only calibration available.

\section{4.c. Relative Efficiency}

Relative efficiency is a term used mainly by the manufacturers of $\mathrm{Ge}(\mathrm{Li})$ detectors to specify their products. In prizciple it is the ratio (in percent) of the absolute efficiency $\left(\epsilon_{T}\right)$ of the $\mathrm{Ge}(\mathrm{Li})$ detector for counting the $1332-\mathrm{keV}$ gamma ray from ${ }^{60} \mathrm{Co}$ at $\mathrm{r}=$ $25 \mathrm{~cm}$ to the absolute efficiency of a 7.6-cm-diam by 7.6. $\mathrm{cm}$-thick NaI at the same source-to-detector distance. In actuality the comparison is usually to the calculated absolute efficiency of a standard $\mathrm{NaI}$ rather than a comparison with a real detector. This is discussed more fully in Sec. 3.5.

\subsection{Types of Gamma-Ray Detectors}

The two types of gcmma-ray detectors present.y in greatest use in fissionable material assay are sodium iodide or cesium iodide scintillation defectors and solid-state germanium detectors. The low detection efficiency of gas proportional detectors makes their use impractical except in high-flux environments. Similarly, the low detection efficiency of silicon solid. state detectors limits their usefulness primarily to $x$ ray detection. Although the relatively new $\mathrm{CdTe}$ semiconductor detectors may prove useful in nuclear safeguards applications in the near future, they are not in general use at this time. Chapter 3 contains more delailed information on the properties of semiconductor and scintillation detectors. For more information, the book Applied Gamma-Ray Spectrometry ${ }^{2.1}$ is highly recommended. Several detector manufacturers have also published booklets on detector theory and operation. ${ }^{2.2}$

\section{5.a Scintillation Detectors}

A scintillation detector consists of a luminescent material, called a phosphor, optically coupled to a photomultiplier tiabe. The elecirons produced by the interactions of gumma rays in the phosphor ercite the atoms and molecules of the phosphor; the subsequent deexcitution results in the envission of fluorescent ultraviolet and visible ligiti radiation. 
The directions of the emitted light photons are random, therefore the phosphor is usually surrounded by a reflector to maximize the number of photons collected by the photosensitive cathode of the photomultiplier tube. The electrons emitted by the ohotosensitive cathorle produce additional electrons by secondary emission within the photomultiplier and are eventuaily collected on the photomultiplier anode to produce an electrical pulse. This pulse generally undergoes amplification and shaping by some type of external electronic amplifier. If the final pulse height is proportional to the incident gammaray energy, the system can be used as a scintillation spectrometer.

Figure 2.7 shows a drawing of a typical scintillation detecto: The most common scintillation detector in use for nuclear material assay is $\mathrm{Nal}$. A complete scintillation spectrometer generally consists of a scintillation crystal-photomultiplier tube assembly (the detector), some type of electronic amplifier to amplify the photomultiplier pulses, a high-voltage source for the photomultiplier (usually 1000 to $2000 \mathrm{~V}$ is required), and some type of pulse-height analyzing circuitry. The complexity of the pulse-height analyzing part of the system ranges all the way from a simple scaler, that counts all of the pulsos from the detector above some threshold level, up to a cc nputercontrolled multichannel pulse-height anaiyzer. Portable systems useful for safeguards applications are now available commercially. The simplest of these systems has a detector and a small box containing a high-voltage power supply, amplifier, single-channel pulse-height analyzer, scaler, and timer. The unit may be powered from normal $110-\mathrm{V}$ ac or a rechargeable battery pack. A slightly larger unit is also available that contains a second single-channel analyzer for background measurement, and stabilization circuitry to minimize the effects of temperature changes. (The light output of scin-

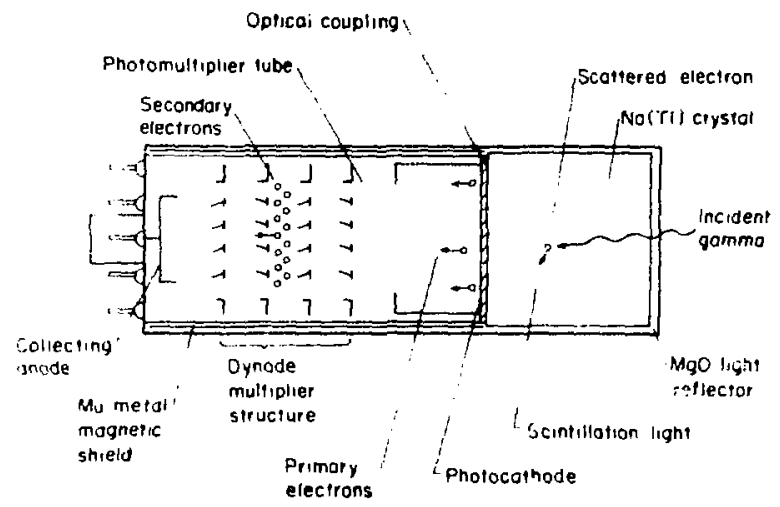

Fig. 2.7.

The scintillation detector. tillators and the gain of photomultiplier tubes are both temperature sensitive.) The use of these instruments in practical assay applications will be discussed in a later section.

\section{5.b Semiconductor Detectors}

The operating orinciple of semiconductor radiation cotectors is completely different from that of scintillaticn detectors, although the end result is the acrne; nomely, the production of an electrical pulse in response to interactions of nuclear radiation withisi the detector volume. A semiconductor detector can be thought of as the solid-state equivalent of a gas ionization detector. Interactions of nuclsar radiation with the detector material give rise to free-charge carriers that are swept through the material by an applied electric field producing an electrical pulse.

The attractiveness of semiconductor detectors results from their improved energy resolution compared to scintillation detectors and from the increased stopping power for radiation of $\alpha$ solid compared to a gas. Basically, the reason for the improved energy resolution lies in the fact that less energy is required to produce $\alpha$ given number of free-charge carriers.

For simplicity, we assume that the energy resolution of $\alpha$ detector is determined solely by the statistical fluctuations in the number of charge carriers produced for a given energy deposition. In a semiconducior defector made from silicon or germonium, about $3 \mathrm{eV}$ is required to produce one freecharge carrier (called an electron-hole pair); for a gat ionization detector, $30 \mathrm{eV}$ is required to produce one electron-Fnsitive ion pair. Scintillation detectors suffer from the inefficient multistep scintillation process, and about $30 \mathrm{eV}$ is required to emit one electron from the photocathode.

The total amount of charge liberated, $Q$, bi the deposition of a given amount of energy, $E$, in a delector is given by

$$
Q=(E / \epsilon) q \text {. }
$$

where $t$ is the energy required to produce a singlecharge carrier (electron-hole pair, electron-positive ion pair, or photoelectron), and $q$ is the charge of on electron. If the creation of charge carriers is purely random, then the standard deviation in the number of carriers produced, $\sigma$, is given by

$$
\sigma=\sqrt{\mathrm{E} / \epsilon}
$$


A measure of the energy resolution obtainable is given by

$$
\sigma / Q=\sqrt{\epsilon / E} \cdot(1 / q)
$$

According to this simple theory, the resolution of a semiconductor detector should be about 10 times better than that obtainable from a scintillation detec. tor. In fact, the improvement in resolution obtainable from semiconductor oves scintillation detectors can be greater than suggested by the above argument because the fluctuation in the number of electronhole pairs is less than given by Eq. (2.11). An additional degradation in the energy resolution of a scintillation detector is produced by small statistical fluctuations within the photomultiplier tube. Other factors affecting the energy resolution of germanium gamma-ray detectors are discussed in Chap. 3.

The semiconductor detector most commonly used today is the lithium-drifted germanium or $\mathrm{Ge}(\mathrm{Li})$ detector. These are available with resolutions of 1 $\mathrm{keV}$ or less for $186-\mathrm{keV}$ gamma rays compared with $22 \mathrm{keV}$ for the best NaI detector. The major disadvantage is that they must be maintained and operated at cryogenic temperatures and they require vacuurn containment and liquid nitrogen cooling.

A complete germaniun spectrometer consists of the same basic components as a scintillation spectrometer: detector, high-voltage power supply, pulse amplifier, and pulse-height analyzer. However, the pulse amplifying and pulse analysis equipment tend to be more complex to take advantage of the resolintion capabilities of the detector. In particular, more effort goes into designing components of the system for high stability and low noise than is required for a scintillation spectrometer. A multichannel pulseheight analyzer is normally required for spectra analysis, rather than a simple single-channel analyzer, although in some safeguards applications such complexity is not required. Although the components of a germanium spectrometer are more complex, the interpretation of the data obtained by such a system is often simpler than from a scintillation spectrometer because ite greater resolution considerably reduces problems caused by background radiation.

\section{5.c. Ultrahigh Purity Germanium Detectors. (UHP Go)}

If germanium crystals could be grown with sufficiently low impurity levels, the lithium-drift process would not be required to produce good detectors.
Such material has become available within the last few years, and small planar detectors fabricated without using the lithium ion drift process are now available from at least three commercial sources. These detectors are available with sensitive volumes up to about $1 \mathrm{~cm}^{3}$, and are used primarily for $\mathbf{x}$-ray and low-energy gamma-ray detection. The utrahigh purity germanium required for such defectors is still very expensive, which is the main reason that laxger detectors are not presently being produced commercially. However, reports show that UHP Cie detectors have been fabricated in various laboratories with volumes in the 10 to $30-\mathrm{cm}^{3}$ range.

UHP Ge detectors need not be stored at low temperatures as do $\mathrm{Ge}(\mathrm{Li})$ detectors, which is a distinct advantage in many situations. However, UHP $\mathrm{Ge}$ spectrometers must be operated at low temperatures (usually $L_{2}$ ) to sufficiently reduce leakage currents. They are therefore packaged in the same manner as $\mathrm{Ge}(\mathrm{Li})$ detectors, and their operation trom the user's point of view is the same except for one important point-forgetting to fill the $\mathrm{LN}_{2}$ dewar will not result in damage to a detector worth several thousand dollars! A note of caution, however-avoid applying bias voltage to a UHP Ge detector at room temperature because it may damage the preamplifier.

\section{REFERENCES}

2.1. F. Adams and R. Darns, Applied Gamma-Ray Spectrometry, (Pergamon Press, Inc., NY, 1970). This book contains a wealth of information on gamma spectrometry. The first three chapters cover gammaray emission and interaction, and the properties and characteristics of scintillation and semiconductor detectors. Chapter 5 deals with associated detector instrumentution. The appendires include many usetul tables, graphs, and spectra [e.g., x-ray absorption edges and emission energies (table); NaI gamma-ray spectra (over 250 actual pulse-height spectra); $\mathrm{Ge}(\mathrm{Li}$ ) spectra (over 200); intrinsic efficien. cies of NaI detectors (tables); and tables of gammaray energies]. This is a useful reference for the gam. ma assayist.

2.2. "Harshaw Scintillation Phosphors," (Harshaw Chemical Company, Solon, Ohio, 1962); and "Ge(Li) Handbook," (Princeton Gamma-Tech, Inc., Princeton, NJ, 1970), 3rd Ed. These are semipromotional monographs published by two commercial detector manufacturers. However, they contain very readable, simple descriptions of detector properties, sample spectra, resolution, efficiency, and operation. 


\section{GAMMA-RAY DETECTORS II}

Chapter 2 discussed general characteristics of gamma-ray detectors. This chapter discusses in detail the specific properties of the alkali-halide scintillation detectors and germanium semiconductor detectors. The subjects discussed include detector materials, construction, and operation. This is information for the nuclear assayist who desires more background material about the detectors in use. Most of this material will not be covered in the formal course, but can be used as a source of reference.

\subsection{Scintillator Metozials}

The phosphor used in a scintillation detector may be organic ur inorganic. Organic phosphors may be crystals, such as anthracene, plastics, or liquids. Inorganic phosphors are usually alkali-halide crystals, such as $\mathrm{NaI}$ and $\mathrm{CaI}$, in which luminescent centers have been ac ivated by impurities, such as Tl, introduced into the crystal lattice. Other inorganic materials, such as $\mathrm{ZnS}$ and certain types of glass, are also useful scintillator phosphors.

Details of the actual fluorescent processes occurring in the phosphor will not be given here. However, one property of the fluoreecent process worth noting is that the intensity of light emission decays exponentially as a function of time. The intensity of the light from a single scintillation event therefore decays from an initial value $I_{0}$ according to
$I=I_{0} \exp (--t / \tau)$. The time constant $\tau$ is a specific property vi each scintillator, with typical vaiues ranging from $10^{-9}$ to $10^{-6}$ s. The wavelength spectrum of the emitted light also varies between phosphors. To obtain efficient collection of the emitted light, it is important that the phosphor be transparent to its emitted light. Zinc sulphide does not satisfy this condition; for this reason, $\mathrm{ZnS}$ scintillators are usually very thin, or consist of $\mathrm{ZnS}$ powder dispersed in ci transparent medium, such as plastic. The emitted spectrum should also correspond to the sensitive wavelength range of the photomultiplier photocathode, which is normally in the near ultranolet-to-blue range of the spectrum.

Table 3.1 lists the properties of some of the more common scintillator phosphors. Organic scintillators are not commonly used for gamma-ray detection because of their small photoelectric absorption coefficients. Sodium and cesium iodide activated with thallium are particularly well suited for gamma-ray detection. The photoelectric absorption coefficients are reasonably large, and their scintillation efficiencies are at least as good as the best organic scintillator, anthracene. $A$ disadvantage of these scintillators is their long time constants, which are highly temperature-dependent.

Thallium-activated sodium iodide is the most widely used scintillator for gamma-ray spectrometry. Sodium iodide is a clear cubic crystal that can be grown to large sizes. Because the material is quite

T'ABLE 3.1

\section{PROPERTIFS OF SOME COMMON SCINTILLATOR PHOSPHORS}

\begin{tabular}{|c|c|c|c|c|}
\hline Scintillator & $\begin{array}{c}\text { Emisgion } \\
\text { Spectrum } \\
\text { Maximum } \\
\text { (A) }\end{array}$ & $\begin{array}{c}\text { Time Constant } \\
(\mu \mathrm{s}) \\
\end{array}$ & $\begin{array}{l}\text { Density } \\
\left(\mathrm{g} / \mathrm{cm}^{3}\right)\end{array}$ & $\begin{array}{c}\text { Scintillation } \\
\text { Efficiency } \\
\text { Relative to } \\
\text { Anthracene } \\
\end{array}$ \\
\hline $\begin{array}{l}\text { NaI(Tl) } \\
\text { CsI(Tl) } \\
\text { CsI(Na) }\left(\mathrm{NaF}_{2}\left(E_{1}\right)\right. \\
\text { Anthracene } \\
\text { Plastics } \\
\text { Liquids }\end{array}$ & $\begin{array}{c}4200 \\
5650 \\
5650 \\
4350 \\
4400 \\
3500-4500 \\
3500-4500\end{array}$ & $\begin{array}{c}0.25 \\
1.1 \\
1.0 \\
0.9 \\
0.032 \\
0.002-0.005 \\
0.001-0.01\end{array}$ & $\begin{array}{l}5.67 \\
4.51 \\
4.51 \\
3.18 \\
1.25 \\
1.06 \\
0.86\end{array}$ & $\begin{array}{c}2.1 \\
1.0 \\
1 . \because-1.8 \\
0.7-1.1 \\
1.0 \\
0.2-0.5 \\
0.2-0.5\end{array}$ \\
\hline
\end{tabular}


hygroscopic, all machining and polishing must be done in a ciry atmosphere, and must be sealed in air. tight containers. Sodium iodide (thallium) crystals may be obtained from several manufacturers in sealed sans with glass windows for coupling to photomultiplier tubes, or the crystal face may be coupled directly to the photomultiplier tube. Such crystals may be obtained in virtually any configuration desired. Although single crystals with linear dimensions larger than about $25 \mathrm{~cm}$ are very expen. sive, cylindrical crystals $60 \mathrm{~cm}$ in diameter and larger have been produced.

Next to NaI(Tl), CsI(Tl) is probably the most used inorganic scintillator for gamma-ray detection. Owing to its higher $Z$ and slightly higher densily, the gamma-ray attenuation of this material is about $\overline{1} . \overline{5}$ times greater than an equivalent size $\mathrm{NaI}(\mathrm{T} T)$ scintillator. Cesium iodide is not hygroscopic, and can therefore be more easily machined and packaged than NaI. It is also softer and more plastic than $\mathrm{NaI}$, and can withstand severe mechanical shocks and large temperature gradients. Fesium iodide (thallium) is considerably more expensive than $\mathrm{NaI}(\mathrm{T})$ ), so that its general use is not justified economically. However, in instances where higher detection efficiency for a fixed size or resistance to thermal or mechanical shock is an important consideration, this material may be preferable to $\mathrm{NaI}$ (TI) in spite of its increased cost.

A promising scintillator made commercially available within the last few years is sodiumactivated cesium iodide. It has good light output, is much less hygroscopic, and is usable at higher temperatures than $\mathrm{NaI}(\mathrm{Tl})$. However, it is also more expensive.

Europium-activated calcium fluoride scintillators are also used to some extent. These crystals have many properties that provide advartages over other commonly used scintillators for X-ray and chargedparticle detection, but the material's lower $Z$ is a distinct disadvantage for gamma-ray detection. Other scintillator materials, such as zinc sulphide, lithium iodide, glass, and boron compounds, are utilized for the detection of alpha particles and neutrons but are not generally useful for gamma-ray detection.

\subsection{Scintillation Light Collection}

The light collection on the photomultiplier tube photocathode should be as efficient as possible. For this reason, the scintillator is usually surrounded by a reflector, and one side is optically coupled to the glass of the photomultiplier. In some cases a light guide between the scintillator and photomultiplier, usually consisting of polished plastic rods or strips, may be required. The photocathode must be sensitive in the wavelengtin region emitted by the scintillator, and should be uniformly sensitive over its area. Within the photomultiplier tube, the electrons emitted from the photocathode must be collected onto the multiplying elements, called dynodes. The photomultiplier tuhe should be encased in a mugnetic shield to reduce the effects of stray magnetic fields upon the electrons emitted from the photocathode.

The most commonly used reflecting rnaterials are aluminum metal, magnesium oxide, and alumina. The particular material used depends upon the particular scintillator configuration, and is usually a compromise between light collection efficiency and convenience.

The design of the can surrounding the scintillator depends upon the particular application and to some extent upon the scintillator material. Sodium iodicie (thallium), for example, requires a moisture-proof can whereas CsI(Tl) does not. For gamma-ray spectrometry, it is generally desiroble to minimize the mass of the can to reduce distortions in the pulseheight spectrum caused by gamma-ray interactions in the can material. Aluminum is usually used for the cans around $\mathrm{NaI}(\mathrm{Tl})$ and $\mathrm{Cs}(\mathrm{Tl})$, although stcinless steel is sometimes used in applications requiring more rugged construction. Cans with thin beryllium entrance windows are frequently used in applications involving the detection of beta particles or low-energy $x$ rays. Another major consideration in the choice of can material is the prublem of radioactive contamination, particularly in very low-level counting applications. In applications requiring very low backgrounds, aluminum should be avoided because it invariubly contains trace amounts of rodium and uranium. However, backgrounds induced by the can material are generally not a problem in safeguards applications.

The purpose of the optical coupling between the scintillator and photocathode is to reduce the light loss that would normally occur by reflection from the scintillator-glass interface. To be effective in this respect, the material used to join the scintillator to the phototube should have a lower refractive inder than the window of the phototube. Transparent fluids such as mineral or silicone oils are frequently used as the coupling medium. A clear silicone grease, such as Dow Corning C-2.0057, is preferred because it does not flow out of the interface. In some applications, a clear epoxy is used to join the scintillator to the phototube; however, the resulting rigid interface leads to possible thermal expansion problems. 
The photomultiplier tube is the component of scintillction detectors that has undergone the graatest im. provement in the last decade or so. Prusent-day photomultiplier tubes have highly sensitive and uniform photocathodes and relatively low thermionic noise.

\subsection{Semicunductor Detector Matoriale}

For a material to be useful as a solid-state ionization detector, it must be possible to collect the charge produced by the interacting radiation. Furthermore, there must be no large leakage currents in the material as a result of applying the electrical field required to collect the charge. The collection of the charge carriers requires that they not interact with atoms in the crystal during the time required for them to be swept to the collecting electrodes. Consequent$l_{y}$, the collection time must be small compared to the lifetime of the charge carriers. This condition is met only in nearly perfect nonmetalic crystals.

The above requirement places severe limitations on the materials that can be used to fabricate solid. state ionization detectors. Table 3.2 lists the properties of some successfully used materials. Silicon and germanium single crystals are the only available materials with the required high purity for routine fabrication of high-quality detectors. Because of its higher atomic number, germanium is preferred over silicon for gamma-ray detection. Research for using higher $\mathrm{Z}$ materials for gamma-ray detectors is being pursued actively. Cadmium telluride is presently the most promising material, although only relatively small detectors can be successfully fabricated now because of charge carrier trapping problems. However, such detectors have found applications in nuclear medicine because of the high gamma-ray sensitivity obtainable in a small volume. The possible

\section{TABLE 3.2}

\section{PROPERTIES OF SOME SEMICONDUCTOR DETECTOR MATERIALS}

\begin{tabular}{|c|c|c|c|c|}
\hline Property & $\mathrm{Si}$ & Ge & GaAs & CdTe \\
\hline $\begin{array}{l}\text { Atomic number } \\
\text { Density }\end{array}$ & $\begin{array}{l}14 \\
2.33\end{array}$ & $\begin{array}{l}32 \\
5.33\end{array}$ & $\begin{array}{l}32^{a} \\
5.32\end{array}$ & $\begin{array}{l}50^{\alpha} \\
5.86\end{array}$ \\
\hline $\begin{array}{l}\text { Dielectric constant } \\
\text { Energy per hole- } \\
\text { electron pair }(e \mathrm{~V})\end{array}$ & $\begin{array}{l}12 \\
3.6\end{array}$ & $\begin{array}{l}16 \\
2.9\end{array}$ & $\ddot{4.5}$ & $\ddot{4.6}$ \\
\hline
\end{tabular}

affective Z. use of CdTe in some safeguards applications is also being investigated.

The following discussion will be limited to germanium gamma-ray spectrometers constructed from lithium-drifted germenium, $\mathrm{Ge}(\mathrm{L}, \mathrm{i})$, or ultrahigh purity (intrinsic) germanium.

\subsection{Iithium-Drifted Germanium Detec- tors-Ge(Li)}

To understand why the lithium ion drift process is used, it is necessary to explore briefly the effects of impurities present in the germanium crystal. For the present discussion, impurities may be classified as being eithex "donors" or "acceptors," depanding upon whether they have more or fewer valence electrons than germanium. When a valence electron of germanium is excited into the conduction band of the crystal, it leaves behind a "hole" in the valence band. Both the electron and the hole propagate through the crystal until they are neutralized in some manner. In a solid-state detector, such neutralization occurs by collecting electrons on a positively charg. ed electrode and injecting electrons into the holes from a negatively charged electrode. The effect of donor impurities is the trapping of holes, and the effect of acceptors is the trapping of electrons. A major requirement for a material to be useful as a solidstate detector is its ability to collect the charge carriers, therefore the concentration of "uncompen. scted" donor or acceptor impurities must be extremely low.

Germanium (and silicon) crystals cen be grown that are very pure excepic for a low concentration of uniformly distributed acceptor impurities. The trapping centers that result from these acceptor impurities may be compeneated for by using a mobile donor impurity that migrates to these trapping centers, thereby neutralizing them by donating their own free electrons. Lithium, a very fant diffuser in silicon and germanium, is used for this puxpose. An almost exact compensation of the semicunductor material is achieved by using the lithium ion drift process.

The lithium-drift process will not be discussed here because it is an involved process, and in many respects is still more of an art than a science.

One disadvantage of $\mathrm{Ge}(\mathrm{Li})$ detectors is that they must be stored at low temperatures $\left(-20^{\circ} \mathrm{C}\right.$ or below) to prevent the lithium ions from thermally diffusing thereby producing uncompensated regions in the crystal. Germanium (lithium) detectors left at room temperature for any appreciable time will no longer function. In moat inutances, if a detector is warmed 
up for a short time, repairs can be made by lithium redrifting. Redrifting exn be done by the detector manufacturer for about $\mathbf{2 5 \%}$, or less, of the price of a new detector. The usual procedure is to store and operate $\mathrm{Ge}(\mathrm{Li})$ detectors at the boiling temperature of liquid nitrogen $\left(-196^{\circ} \mathrm{C}\right.$ at sea level, and approximately $-206^{\circ} \mathrm{C}$ at Los Alamos), which has the advantage of drastically reducing leakage currente in the detector.

Lithium-drifted germanium detectors are normally housed in a vacuum-insulated stainless steel cryostat that resides in a liquid nitrogen $\left(\mathrm{LN}_{2}\right)$ storage dewar. Occasionally, the detector cryostat is an integral part of the dewar. An aluminum cap usually covers the germanium crystal. The crystal is affixed to $\alpha$ copper "cold finger" that extends from the end of the cryostat into the liquid ritsogen. Several different crystal geometries are available for ditferent applications.

Detectors are commercially available with volumes ranging from $100 \mathrm{~mm}^{3}$ or less up to abcut $100 \mathrm{~cm}^{3}$. Good quality detectors with volumes greater than about $50 \mathrm{~cm}^{3}$ are still difficult to produce and are therefore expensive. The factors that presently limit detector size are the availability of large germanium crystals sufficiently pure and defect-free, and problems associated with drifting lithium ions to depths greater than 1 to $1.5 \mathrm{~cm}$ (drift depths of $\sim 2 \mathrm{~cm}$ are achievable, however).

Detectors are described as being planar, true coaxial, or wrap-around coarial, depending upon how the lithium-drift process is carried out. In planartype detectors, lithium is diffused into one face of a germanium alab, unually cut from a cylindrical cryvial, and cirifted into the material from that face. The face into which lithium was diffused is used as one electrode (positive) and the other face as the other (negative) electrode. In coaxial geometries, lithium is diffused into the sides of the cryotal, usually a right circular cylinder. Wrap-around, or closed end, coaxial detectors are made bv also diffusing lithium into one crystal face. After the lithium-drift process, a small core of uncompensated germanium protrudes through one or both faces of the crystal, and is used as the negative electrode of the detector. Only the compeneated part of the cryatal is useful for detecting radiation (referred to as the sensitive volume), therefore it is desirable to minimize the size of the uncompensated core present in coarial crystals, and the amount of uncompensated material left at the rear face of a plana: detector. At present, detectors with volumes greater than 1 to $5 \mathrm{~cm}^{3}$ are coaxial, with the larger ones usually being the wraparound type. In eafeguards applications, wrap- around coaxial detectors with volumes of the order of 25 to $50 \mathrm{~cm}^{3}$ are generally appropriats. Using larger detectors in such applications is not justified economically.

\subsection{Detection Efficiency}

Although early $\mathrm{Ge}(\mathrm{Li})$ detectors suffered from low detection efficiency (as do presently available UHP Ge detectors) because of their small size, the larger detectors presently available compare favorably with the smaller sodium iodide detectors. For example, a $5-$ by $5-\mathrm{cm} \mathrm{Ge}(\mathrm{Li})$ detector with an active volume of $90 \mathrm{~cm}^{3}$ has about two-thirds the intrinsic full-energy peak detection efficiency of a 5- by $5-\mathrm{cm} \mathrm{NaI(T)}$ detector at $1.33 \mathrm{MeV}$. Manufacturers normally specify detection efficiency in terms of the absolute efficiency of a 7.6- by 7.6.cm NaI(TI) detector for the 1.33-MeV gamma ray from a point ${ }^{60} \mathrm{Co}$ source located $25 \mathrm{~cm}$ from the detector face. In actual practice, no such comparison is made, but rather the $a b$. solute efficiency of the $\mathrm{Ge}(\mathrm{Li})$ detector in question is measured for a ${ }^{60} \mathrm{Co}$ source of known strength located $25 \mathrm{~cm}$ from the face of the germanium crystal, and the result is multiplied by $8.33 \times 10^{4}$ to obtain the percent efficiency relative to a 7.6- by 7.6$\mathrm{cm} \mathrm{NaI(T)}$. Such a relative efficiency value for the $90-\mathrm{cm}^{3} \mathrm{Ge}(\mathrm{Li})$ described above would be about $20 \%$; tor a $30-\mathrm{cm}^{3}$ detector, it would be around $5 \%$.

For high-efficiency germanium spectrometry, it is not only necessary that the sensitive volume be large, but an optimum shape is also required. A thick detector with a small frontal area will be less efficient than a thinner detector of the same volume having a greater area. In safeguards applications, one usually is interested in detecting the $186-\mathrm{keV}$ gamma rays from ${ }^{235} \mathrm{U}$ or the $414 . \mathrm{keV}$ gamma rays from ${ }^{239} \mathrm{Pu}$, which does not require a detector thickness greater than 2 to $3 \mathrm{~cm}$. The lose in total absolute detection efficiency that would result from using a $30-\mathrm{cm}^{3} \mathrm{Ge}(\mathrm{Li})$ detector (2-cm-thick) rather than a $50-\mathrm{cm}^{3}$ detector (4-cm-thick) is about $17 \%$ for $186-k e V$ gamma rays and about $37 \%$ for $414-k e V$ gamma rays, but the sav. ing in cost is almost a factor of two. The use of a thinner detector also reduces the brakground from high-energy guinma rays, as, for example, from the 1. $\mathrm{MeV}$ gamma ray occurring in ${ }^{238} \mathrm{U}$; the total detection efficiency of a $2-\mathrm{cm}$-thick $\mathrm{Ge}(\mathrm{Li})$ detector is about one-half of that of a $4-\mathrm{cm}$-thick detector at 1 $\mathrm{MeV}$.

Figure 3.1 showe the absolute efficiency of a typical large-area $30-\mathrm{cm}^{3} \mathrm{Ge}(\mathrm{Li})$ defector as a function of energy. The sharp drop-off in efficiency 


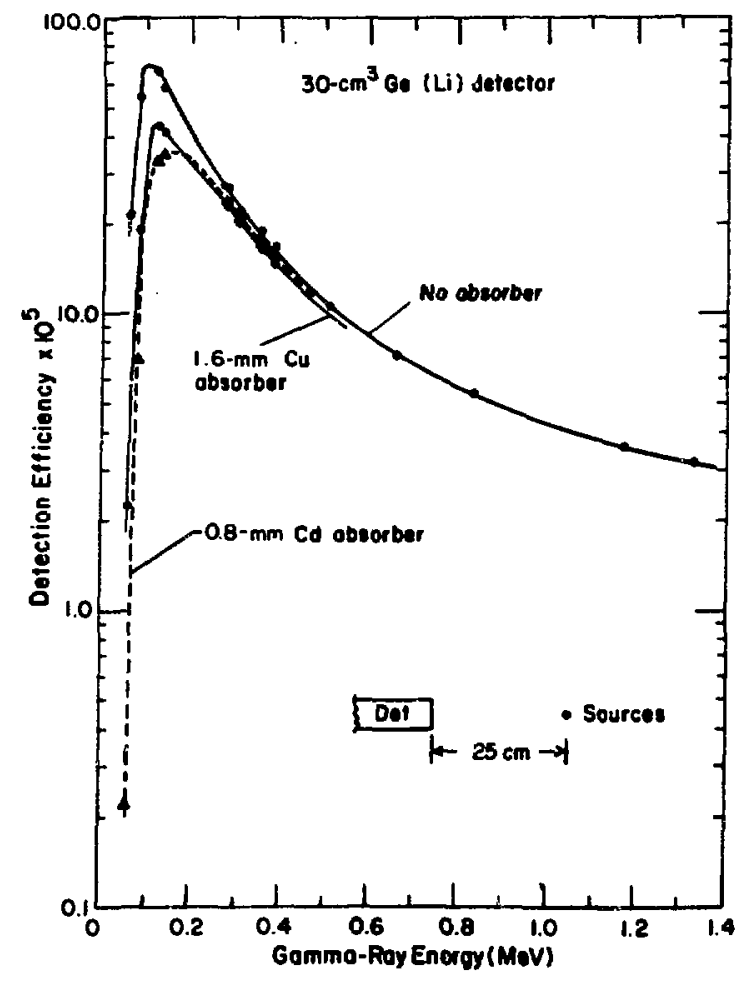

Fig. 3.I.

Absolute efficiency vs energy for $30-\mathrm{cm}^{3} \mathrm{Ge}(\mathrm{Li})$ detecior.

below $100 \mathrm{keV}$ result from absorption in a thin (0.3to $0.5 . \mathrm{mm}$ ) "derd layer" on the detector aurface that result from the lithium-drift process. In mall planar detectors derigned for use at energies below $100 \mathrm{keV}$, this deais layer is removed. In plutonium meanurement, a this cadmium absorber is unually placed in front of the detector to atienuate the 59.keV gamma rays from $241 \mathrm{Am}$. The effect of auch an abworber in also shown in Fig. 3.1; a $0.8-\mathrm{mm}$ cadmium absorber reduces the 59-keV detection efficioncy by a factor of 100 , but only reduces the cletection efficiensy at 414 keV by about $3 \%$.

\subsection{Energy Resolution}

The energy resolution obtainable with semiconductor detectors depends not only on the statistical variation in the number of electron-hole pairs collected, but also on the noise generated in the detector and by subsequent pulse amplitiers. $\mathrm{Ob}$. viously these noise sources must be reduced to a minimum. For the detector, noise reduction is mainly a matter of reducing the surface leakage current by proper surface treatment during the manufacturing process. Amplitier noise is produced primarily by leakage currents and capacitance at the input of the firet amplifier stage (the preamplifier), which may be reduced by proper design of the rystem. All presently available preamplifiers dexigned for use with solidstate detectors use tiold-effect transintors (FET's) as the first amplifying element; these devices introduce lese noise into the system than standard transistors or vacuum tubes. In very high-resolution rystems, the FET used in the preamplifier is mounted inside the detector cryontat and cooled to near $\mathbf{L N}_{2}$ temperature. This not only roduces leakage currents within the FET, but also reduces stroy capecitance. Another, often overlooked factor affecting energy resolution is gain instabilities in the pulse cmplifying or processing equipment. The resolution obtainable from a dotocior-amplifier artem thould be considered as a whole, becaves a noiny preamplifiar will severely degrade the performance of a good detector, chd vice verea.

The energy resolution attainable when all noise sources are eliminated is important becanse it provides the theoretical limit of the resolving power of germanium detectors. For this limit to be ap. proached, it is aleo necescary that there be negligible low of charge carriers in the detector due to trapping and recombination. The "theoretical limit" of detector resolution has become less, over the last fow years, as bettes quality germanium crystals have become available-deiector syutems are now available commercially that have resolutions better than the theoretical limit of 5 or $6 \mathrm{yr}$ ago!

The effects that various noise sources have on system resolution are determined largely by pulse shaping within the amplifier. For scintillation detectors, the main need for pules shaping in the amplifier is to reduce pile-up effecte. For solid-atate detectors, an additional consideration is the effect of pulese shaping on the overall system resolution. The pulse shaping network act as frequency filters, and because different noise sources have different trequency spectra, a retwork can be chosen, in principle, that will give optimum resolution for a particular oystom. In practice, the type of pulse shaping uetwork used is a compromise between such factors as noise reduction, pile-up effects, ease of implementation, overload recovery, and in some cases time resolution. The type of shaping network now used in most good quality linear ampiifiers designed for solid-state detectors is referred to as Gaussian or modified Gaussian. This type of network produces a fairly symmetrical pulse, has good overload recovery characteristics, and is close to on ideal filter for the types of neise spectro associated with most solid-state detector-preamplifier combinations. The shaping 
time constants used range from 1 to $10 \mu \mathrm{B}$, with the shorter time constants generally used with the larger coaxial dotectore and the longer time constants used with imall planar detectors at low count rates. As the counting rate increases, it is uvually advantogeous to decrease the pulse-shaping time constant to reduce pile-up offects. With mont linear amplifiors, from three to tive different time constants can be selected by a front panol switch.

Mont present-day linear amplifiere incorporate what are called "pole-zero" cancsllation networks and bose line restoration networks. These notworks are adjustable, to some extent, oither isy front panel or internal controls, and their adjustmeni procedures are described in the manual supplied with the amplifier. The proper adjuntment of these networks can greatly improve system resolution at high count rates.

Energy resolution of a detector system is normally expresed in terms of the full width at half height (or hall maximum) of a full onergy peak, and is ab. breviated FWHM. The unit of meature may be eithir electron volt (eV) or kiloelectron volts (keV). The FWHM of a full onergy peak from an ideal (noiselese) detector is directly related to the standard deviation in the number of charge carriers produced. Equation (2.11) shows that this quantity is proportional to ihe square root of the incident gamma-ray energy. One would thesefore expect, for an ideal detector, that a fourfold increase in energy would result in a twofold increase in the full-energy peak FWHM. In proctice, the FWHM will increces more slowly at low energies due to the presence of noive that set a lowor limit on the attainable FWHM, and may increase more rapidly at unorgies above $\sim 500$ keV due to charge carrior trapping.

Trapping of charge carriors is readily apparent from the dotector's pulse-hoight rpectrum. If trapping is presont, the tull-energy peaks become arymmetric on the low-energy side. This peak arymmetry, or "tailing," tends to docrease and the poak positions shift upward in the spectrum at the detector bias is increased. Complete charge collection tabilizes the peak shape and pocition. As the detector bien is increaned beyond the point required for complote charge collection, the peaks will eventually begin to broaden due to increased leakage currents. A prerequiaite for a good detector in that leakage currents be negligible at the bias level required for complete charge collection. Good detectore generally exhibit constant resolution over an operating voltage range of eeveral hundred volts. Germanium detectorg are normally operated at bias voltages that produce fields in the detector of 150 to $200 \mathrm{~V} / \mathrm{mm}$, at which point the charge carrier velocities saturate at about $2 \times 10^{7} \mathrm{~cm} / \mathrm{s}$.
Figure 3.2 shows the FWIHM of two good-quality $\mathrm{Ge}(\mathrm{Li})$ detectors, one an $80 . \mathrm{cm}^{3}$ coaxial and the other a l- $\mathrm{cm}^{3}$ planar, plotted against the square root of the energy of the full-energy peak. The FET used in the preamplifiers associated with both detectors was cooled to near $\mathrm{LN}_{2}$ temperature. The higher capacitance and surface leakage currents ansociated with the large detector limit its minimum attainable FWrMM to about $800 \mathrm{eV}$, compared to about $300 \mathrm{oV}$ for the anall detector.

Detector manufacturere normally specify the FWHM attainable with particular detectorpreamplifier combinations at one or two energies. For amall planar detector, the FWHM is unually opecified for the 122-keV ${ }^{57} \mathrm{Co}$ gamma ray, and for the 5.9-keV ${ }^{5 F}$ Fe $x$ ray if the detector is intended for uee at low encrgies. The FWHM of coarial detectore is specitied for the $1.33-\mathrm{MeV}{ }^{60} \mathrm{Co}$ gamma ray, and usually for the $122-\mathrm{keV}^{57} \mathrm{Co}$ gamma ray. The FWHM mecoured at $122 \mathrm{keV}$ normally ranges from about 500 -V for high-quality planar detectors up to $1.5 \mathrm{keV}$ or more for largo coaxial detectors with roomtemperature preamplitiers. The FWHM at $1.33 \mathrm{MeV}$ moy range from about $1.6 \mathrm{keV}$ for very good detectore up to $3 \mathrm{keV}$ or more for poor detectors. For most nucleur eafoguards applications, a FWHM at 122 $\mathrm{koV}$ of $1.2 \mathrm{koV}$ or les and a FWHM at $1.33 \mathrm{MoV}$ of $2.3 \mathrm{koV}$ or lese is unually sufficient. Note that coaxial detectors with resolutions les than $2.0 \mathrm{keV}$ at 1.33 $\mathrm{MoV}$ generally coet about $15.20 \%$ more than vimilar detectors with resolutione in the vicinity of 2.2 to 2.5 koV.

Many detector manufacturers aleo specify the full width at one-tenth maximum (FWO.lM) resolution

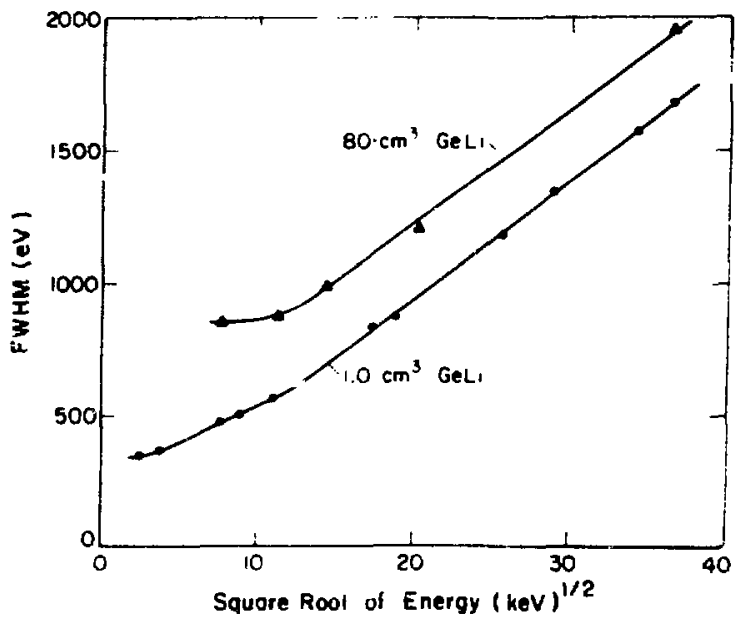

Fig. 3.2.

Resolution vs energ! for two Ge(Li) detectors. 
obtained at $1.33 \mathrm{MeV}$ as a measure of any peak tail. ing present caused by incomplete charge collection. The value of the FW0.1M/FWHM ratio for a detector with minimal charge collection problems will be 1.85 to 1.9.; ration greater than 2.0 indicate noticeable tailing of full-energy peaks, which should generally be avoided. Some detector expert advocate uning the FW0.05M or oven FW0.01M resolution as a more sensitive indicator of peak tailing, but these quan. tities are more difficult to measure. For an ideal detector, the value of FW0.05M/FWHM is 2.079; values in the range 2.1 to 2.3 are obtainable with good detectors. 


\section{ANALYSIS OF GAMMA-RAY PULSE-HEIGHT SPECTRA}

As previously discussed, the electrical pulses produced by scintillation and solid-state spectrometers are proportional to the energy deposited in the detector by ionixing radiation. A multichannel pulse-height analyzer is generally used to digitize the pulses from such spectrometers. The pulse-height analyzer (PHA) typically contains between 100 and 4100 channels, each channel corresponding to a small range of pulse heights. For example, in a 2000 . channel PHA having an input pulse-height range of 0 to $10 \mathrm{~V}$, each channel would correspond to a $10 / 2000-\mathrm{V}$ or $5-\mathrm{mV}$ range of input pulses. The number of pulses that occur with heights falling within the range of each channel is stored in an electronic memory within the PHA. A plot of the number of events, or counts, stored in each channel vs the channel number produces what is termed a pulseheight spectrum.

A PHA is typically an expensive, nonportable in. strument. An alternative method for measuring gamma-ray pulse-height spectra invclves the use of a single-channel analyzer (SCA). This is less expensive and is crailable in portable instruments; however, it is a tedious and time-consuming procedure and can be used only with scintillation detectors (Nal). An SCA accepts the amplified detector pulse and determines if it is within a preset pulse-height range. This range (or window) corresponds to a single channel of a PHA or multichannel analyzer. When a detector pulse is within the selected energy window, the SCA issues an output pulse which is usually fed to a scaler. A pulseheight spectrum can be measured in the following way: set the energy window for a small $\Delta \mathrm{E}(e . g$. , if amplifier range is 0 to $1 \mathrm{~V}$, set $\Delta E=0.01 \mathrm{~V}$ ), set the threshold (E) at 0 and take $a$ short count, record the resulting scaler value, increase the threshold setting and take another count, continue until the upper limit of the threshold control is reached, and, finally, plot the scais counts vg threshold setting. The resulting plot will be identical to that which would be obtained with a 100-channel analyzer; however, it will have taken at least 100 times as long to collect.

The pulse-height spectra obtained from gammaray spectrometers generally contain a series of peaks superimposed on a smoothly varying background. The peaks zepresent gamma rays that have deposited all of their energy in the detector; their positions are a measure of the gamma-ray energies, and the areas under the peaks (above background) are a measure of the gamma-ray intensities. The peak widths are a measure of the system energy resolution, as described previously.

Pulse-height spectra obtained from a $30-\mathrm{cm}^{3}$ $\mathrm{Ge}_{\theta}(\mathrm{Li})$ detector and a 7.6- by 7.6-cm NaI(T) detector exposed to plutonium gamma rays are shown in Fig. 4.1. The superior energy resolution obtainable with a $\mathrm{Ge}(\mathrm{Li})$ spectrometer is clearly evident in Fig. 4.1 ; the $\mathrm{Ge}(\mathrm{L}$ i) pulse-height spectrum is a much better representation of the incident gamma-ray energy spectrum than the $\mathrm{NaI}(\mathrm{TI})$ spectrum. A Ge(Li) pulseheight spectrum from an enriched uranium sample is shown in Fig. 4.2. For comparison, Fig. 4.3 is a NalSCA spectrum of enriched uranium.

In generd, the andysis of such pulse-height spectra consists of determining the peak positions for energy meaurement and the peak areas for intensity measurement. The gamma-ray intensities, and thezefore the peak areas, are directly proportional to the anount of material producing the gamma rays (ignoring, for the moment, absorption within the sample). Only the areas of selected strong peaks are usually of interest in assay measurements. For ${ }^{235} \mathrm{U}$ assay the area under the $185.7-\mathrm{keV}$ peak is normally used. The 413.7-keV peak (and sometimes the 37j$\mathrm{keV}$ peak) is used for ${ }^{239} \mathrm{Pu}$ determination. The $186-$ $\mathrm{keV}$ peak from ${ }^{235} \mathrm{U}$ is reasonably well resolved in a NaI(Tl) pulse-height spectrum. Plutonium gamma rays are clearly not well resolved by a NaI(TI) spectrometer, as shown in Fig. 4.1. Although Nal(Tl) detectors can be (and indeed are) used for plutonium assay, background determination is more difficult than with $\mathrm{Ge}(\mathrm{Li})$, particularly if varying comounts of ${ }^{241} \mathrm{Pu}$ are present.

A simple, yet fairly general, method of determining photopeak areas will now be described. The method is applicable to PHA data, or to data obtained from scalers connected to single-channel analyzers. Figure 4.4 shows a portion of a pulseheight spectrum containing a single photopeak. The area under the peak, P, may be obtained by summing the contents of the channels as shown in the figure. The background under the peak can be approximated by a straight line, shown dashed in the figure. Groups of channels lying on each side of the peak may be averaged to estimate the background. If $n_{1}$ channels are used on the low side of the peak, $n_{2}$ channels are used on the high side, and $n$ 


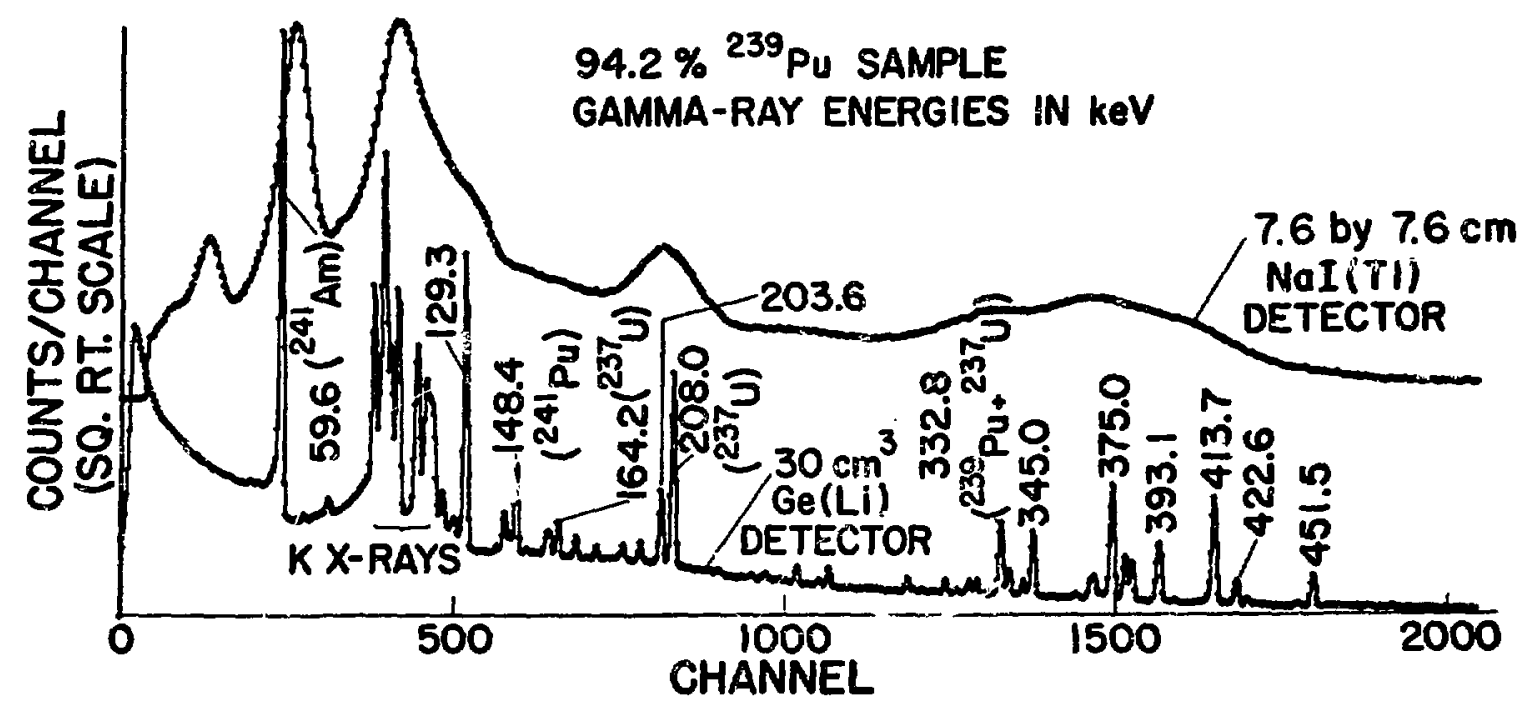

Fig. 4.I.

Cimpurison of plutonium gamma-ray spectra from $30-\mathrm{cm}^{3} \mathrm{Ge}(\mathrm{Li})$ detector and 7.6- by 7.6-cm NaI detector. Note the square root scale.

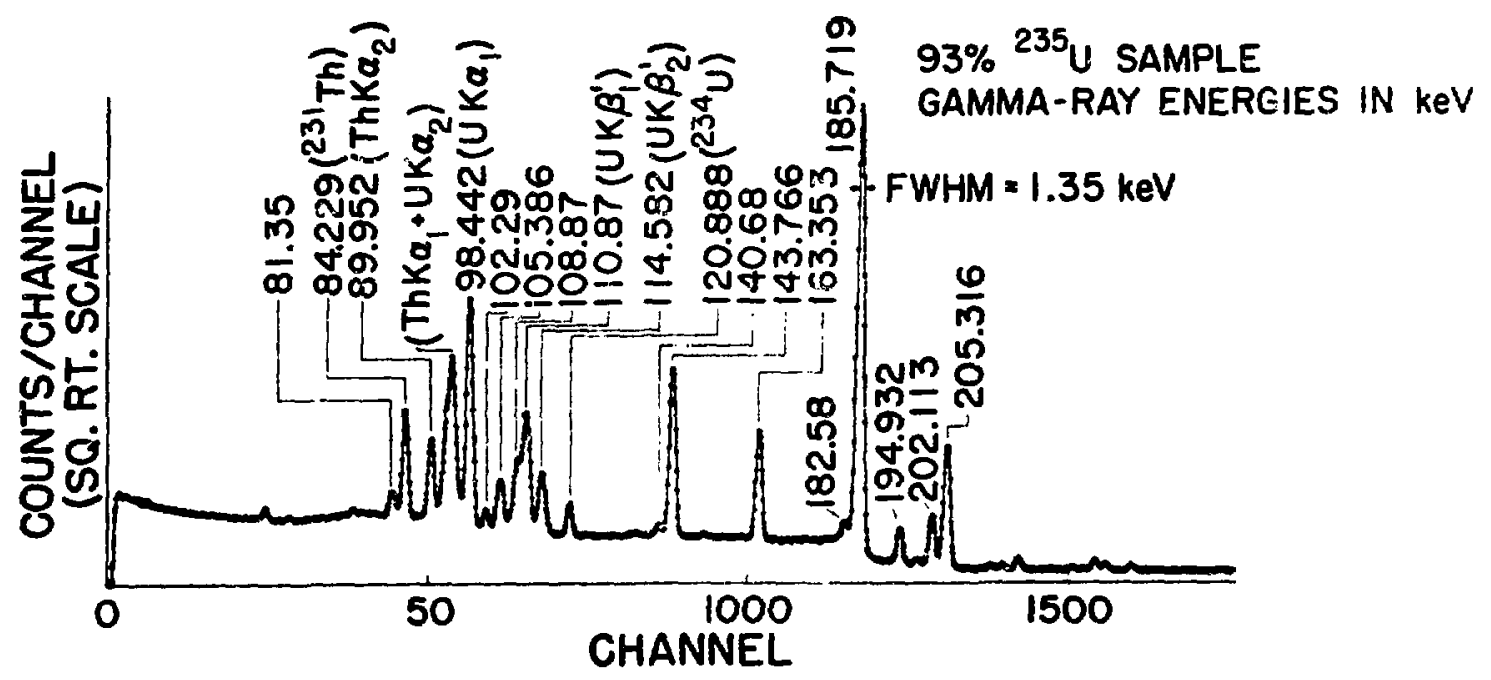

Fig. 4.2.

Enriched uranium gumma-ray spectrum as measured with a high-resolution Ge(Li) detector. 


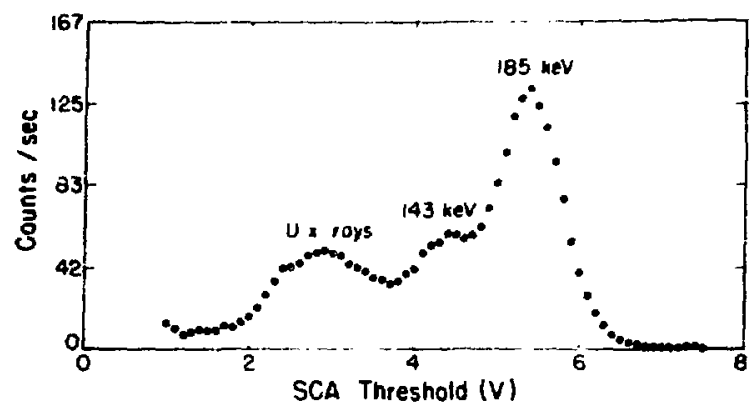

Fig. 4.3.

Enriched uranium gamma-ray spectrum as measured with a $\mathrm{Nal}$ detector. Data were taken with SCA in SAM-II.

channels are ued in the peak, then the beckground is given by

$$
B=(n / 2) \cdot\left(B_{1} / n_{1}+B_{2} / n_{2}\right)
$$

The peak area corrected for background is then given by
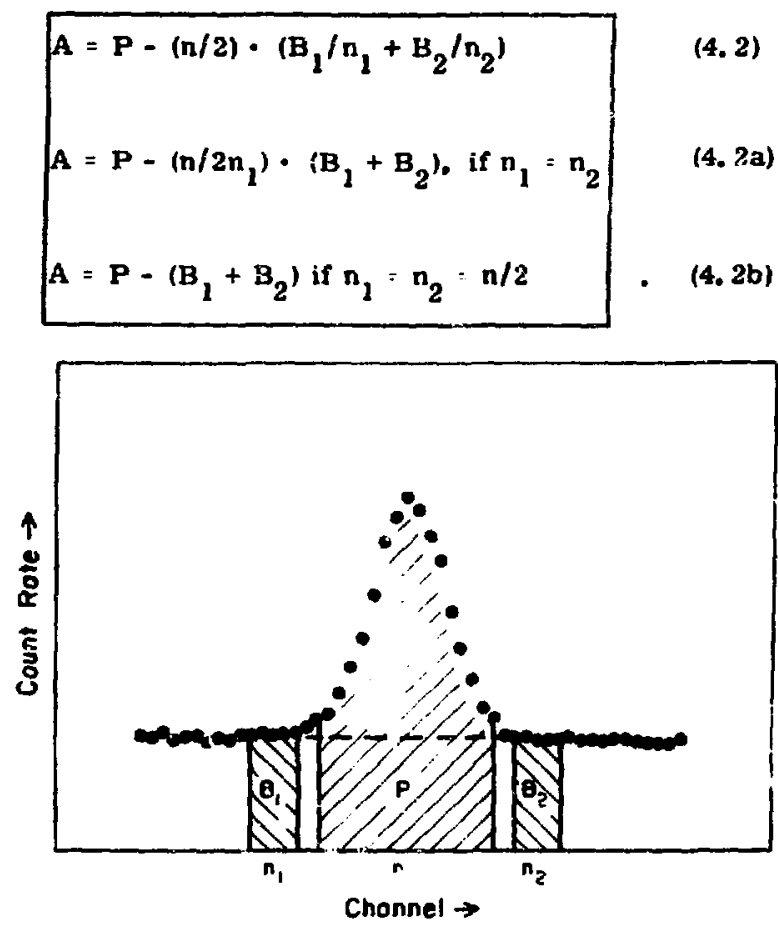

Fig. 4.4.

Pulse-height spectrum of single photopeak itlustrating general procedure for determining photopeak area. The area of interest is above the dashed line which is determined from $B_{1}$ and $B_{2}$.
If the slope of the straight liae background is esseatially constant for a particular set of measurements, then channels on one side of the peak only can be used for the background determination. The peak area would then be given by

$$
A=P-\left(n / n_{1}\right) \cdot(k \cdot B) \text {. }
$$

where $\mathbf{k}$ is a factor that corrects for the background slope ( $k=1$ for a "level" background) determined from an appropriate calibration. This situation is illustrated in Fig. 4.5.

This "two-window" procedure is commonly used with Nal-SCA instrumentation. For this, one SCA window is set over the peak and the other is set alightIy higher in energy. Scalers attached to the SCA's measure $P$ and $B$, respectively. If only one SCA. scaler combination is available, two separate counts can be made ai different threshold settings. The net peaik area then becomes

$$
A=P-(k \cdot B) \text {. }
$$

where $\mathrm{k}$ is determined during the system calibrction.

When the photopeak area has been determined, the stutistical uncertointy of the measured area is also of interest because this gives an estimate of the measurement precision. The standard deviation in the number of counts $N$ registered by a nuclear radiation detector is given by $\sqrt{\mathbb{N}}$. Therefore the standard deviation in $P$, the peak area uncorrected

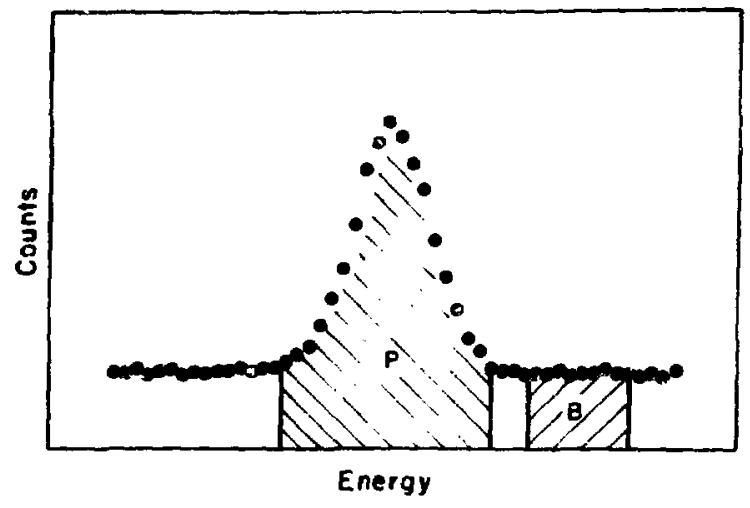

Fig. 4.5.

Pulse-height spectrum of single photopeak iflustrating iwo-window method for determining photopeak area. This is the most common procedure for Nal-SCA measurements. 
for background, is given by $\sqrt{P}$. Without proof, the standard deviation of the measured background is given by

$$
\sigma(B)=\left(n_{1} / 2\right) \cdot \sqrt{B_{1} /\left(n_{1}\right)^{2}+B_{2} /\left(n_{2}\right)^{2}},
$$

and the standard deviation in A [calculated from Eq. $(4.2)]$ is given by

$$
\begin{aligned}
\sigma(A) & =\sqrt{\sigma^{2}(P)+\sigma^{2}(B)} \\
p(A) & =\sqrt{P+(n / 2)^{2} \cdot\left[B_{1} /\left(n_{1}\right)^{2}+B_{2} /\left(n_{2}\right)^{2}\right]} \\
& =\sqrt{P+\left(n / 2 n_{1}\right)^{2}\left(B_{1}+B_{2}\right)} \text { if } n_{1}=n_{2} \\
& =\sqrt{P+B_{1}+B_{2}} \text { if } n_{1} \quad n_{2} \quad n_{2}
\end{aligned}
$$

The standard deviation associated with the twochannel method for SCA's [Eq. (4.3a)] is

$$
\sigma(A)=\sqrt{P+k^{2} B} \text {. }
$$

Two things should be noted from Eq. (4.5).

1. The precision of a measurement can be improved by using more PHA channels or langer SCA windows to determine the background,

2. The precision in the measurement of a small peak situated on a large background will be poor, because $a(A) / A$ will be large. In 1 , the number of channels used tor background measurement should be limited to the insmediate vicinily of the photopeak of interest so that other peaks are not included. As an example of 2 , assume that the following values have been measured.

$$
\begin{array}{ll}
P & 15000, \\
B_{1} & 7500, \\
B_{2} & 6000, \text { with } \\
n & 10, \\
n_{1} n_{2} 5 .
\end{array}
$$

Then from Eq. (4.2b) the peak area is

$$
A=15000-(7500+6000)=1500 .
$$

The standard deviaticn of $A$ is given by

$$
\sigma(A)=\sqrt{P+B_{1}+B_{2}}=168.8 .
$$

The estimate of the measurement precision of $A$ is $\sigma(A) / A$, or about $11 \%$. However, the quantity $P$, the peak area uncorrected for background, was measured with an estimated precision of about $1 \%$ ! $[\sigma(P) / P=\sqrt{15000} / 15000=0.008$. $]$ The precision of the measurement in this case could have been improved only slightly by using twice as many channels to determine the background. For example, if

$$
\begin{aligned}
& B_{1}=15000, \\
& B_{2}-12000 \text {, and } \\
& n_{1}-n_{2} 10 \text {, }
\end{aligned}
$$

then

$$
\begin{gathered}
\sigma(A) / A \sqrt{\mathrm{P}+(1 / 2)^{2} \cdot\left(B_{1}+B_{2}\right) / A} \\
147.5 / 1500=0.098 .
\end{gathered}
$$

oz about $10 \%$. A further discussion of counting statistics is given in Appendix B.

The foregoing method of photopeak analyais assumes that the photopeak of interest is well resolv. ed from neighboring peaks. However, for poorly resolved multiple peaks due to gamma rays arising from the decay of the same isotops, this technique could be used to determine the total area of the peaks for an assay measurement. For unresolved peaks arising trom different isotopes, an assay could be performed on the combined isotopes using this methad, but not on the individual isotopes unless additional information was available from another measuremer:. 
More general methods of andysis have been developed to analyze poorly resolved spectro. Such methods are usually not required in scifeguards ansay of plutonium and uranirsm, and will not be discussed here. Whon mose oxtenuve anclysin is required, it is vaually performed by an on-line computer system. 


\section{QUANTITATIVE GAMMA-RAY ASSAY}

\subsection{Introduction}

The general procedure for gamma-ray assay is outlined in Eq. (5.1).

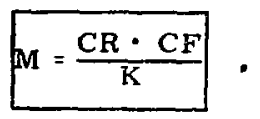

where

$$
\begin{aligned}
& M= \text { mass of isotope of interest } \\
& C R= \text { measured count rate from } \\
& \text { signature of isotope } \\
& C R= \text { correction factor for sample } \\
& \text { attenuation and } \\
& K \quad \text { - calibration factor (corrected } \\
& \text { counts per gram) }
\end{aligned}
$$

The calibration factor $(\mathrm{K})$ is determined by measuring a knowu standard. This can be represented by inverting Eq. (5.1).

$$
\mathrm{k}=\frac{\mathrm{CR}_{\mathrm{s}} \cdot \mathrm{CF}}{\mathrm{M}_{\mathrm{s}}} \text {. }
$$

where

$$
\begin{aligned}
& M_{s}=\text { known mass of emitting } \\
& \mathrm{CR}_{\mathbf{S}}=\text { measured coint rate from } \\
& \text { standard, and } \\
& C F_{\mathbf{S}}=\text { correction factor for attenuation } \\
& \text { in standard. }
\end{aligned}
$$

The standard essentially provides a measurement of the detector efficiency, the specific activity of the signature of interest, and the effects of sample geometry (size, shape, and sample-to-detector distance). These equations assume that the standard is the same shape and is measured in the same position as the unknowns. If not, corrections must be applied for the effect of different sample-to-detector distances. The above ideas may be stated simply, but the assayist needs a good understanding of the many factors involved to apply them correctly. The following is a list of important factors to be considered when attempting gamma-ray assay of fissionable material.

1. Gamma-Ray Signatures. The assarist must know the energies and intensities of the relevant gamma rays. These place fundamental restrictions on the sensitivity, precision, and accuracy of any assay. This information is listed in Table 1.1 and Appendix A. The important formulas are

$$
N(t)=N(0) e^{-\lambda t}
$$

where

$$
\begin{aligned}
& \lambda=0.693 / \mathrm{t}_{\frac{1}{2}} \text { (half-life) ; } \\
& R=\frac{1.32 \times 10^{16}}{\mathrm{At}_{\frac{1}{2}}}(\mathrm{y} / \mathrm{g}-\mathrm{s}), \mathrm{t}_{\frac{1}{3}} \text { in years }(1.4)
\end{aligned}
$$

2. Detecturs and Gamma-Ray Spectra. A qualitative understanding of detector properties and the general appearance of pulse-height spectra are necessary to interpret the output data. This is discussed in Chaps. 1 and 2. The material in Sec. 2.1 (especially Fig. 2.1) is of particular importance.

\section{Spectral Peak Stripping and Backgro:ind Subtrac-} tion. These are the basic prccedures for extracing information from the measured spectrum. The assayist must be very familiar with the material in Chap. 4, especially the cpplication of Eqs. (4.2)-(4.6), which include the two-window SCA procedure.

$$
\text { A } P-(k \cdot 13) \text {, }
$$


where

$$
\begin{aligned}
A & =\text { net counts } \\
P & =\text { counts in peak SCA } \\
B & =\text { counts in background SCA; } \\
\sigma(A) & =\sqrt{P+k^{2} B} .
\end{aligned}
$$

4. Detector Efficiency and the Inverse Square Law. This is covered in Sec. 2.4. Section 5.2 contains further discussion of how $1 / x^{2}$ affects gammaray assays. The important formulas here are

$$
\epsilon_{p} \approx 1-e^{-\mu_{l} t} \quad \text { photopeak efficiency, (2.3) }
$$

where

$$
\text { t : detector thickness, and }
$$

$\mu_{l}=$ detector attenuation coefficient.

$$
\epsilon_{T}=\frac{A \cdot \epsilon_{p}}{4 \pi r^{2}} \quad \text { absolute efficiency } \cdot(2.8)
$$

where

$$
A=\text { visible detector area. }
$$

5. Attenuation of Gamma Rays. The discussion in Chap. 1 is reempharized and extended in Sec. 5.3. The assayist should be able to make simple com. putations of attenuation and should know the values of some important mass attenuation coefficients. The important formulas are

$$
1=1_{0} e^{-\mu_{l} L} .
$$

where

$$
\begin{aligned}
& \mu_{\ell}\left(\mathrm{cm}^{-1}\right)=\text { linear attenuation coefficient : } \\
& \mu_{\mathrm{m}}=\frac{\mu_{l}}{\rho},
\end{aligned}
$$

where

$$
\mu_{m}\left(\mathrm{~cm}^{2} / g\right)=\text { mass attenuation coefficient } .
$$

6. Altenuation Correction Factors. The attenuation correction is probably the most important factor in Eq. (5.1), and will be discussed in delail in Sec. 5.4.
5.2. Gamma-Ray Assay and the Inverse Square Law

The inverse square law of gamma-ray flux was discussed in Sec. 2.1. The basic formula for absolute efficiency for a point source is given as

$$
\varepsilon_{T}=\frac{A \cdot \varepsilon_{p}}{4 \pi r^{2}},
$$

where

$$
\begin{aligned}
& A=\text { visible detector area }, \\
& \boldsymbol{E}_{\mathbf{p}}=\text { detector photopeak efficiency }, \text { and } \\
& \mathbf{r}=\text { source-to-detector distance } .
\end{aligned}
$$

Most samples for fissionable material assay are extended sources, so that $r$ and $\epsilon_{T}$ vary from one point to another on the sample. This means $\mathrm{l} g$ of uranium may yield different count rates depending on its location within the sample. Consider the cross section of a $55 . \mathrm{gal}$ drum (diam $=60 \mathrm{~cm}$ ) illustrated in Fig. 5.1 . One gram of material in position 2 counts 4 times as much as in position 4. If all somples were uniform, this variation of response (count rate) with position would be uximportant. Because many samples are not uniform (particularly the waste stored in 55-gal drums), this represents a potential source of error that can be minimized. This can be accomplished by increasing the sample-to-detector distance, but only at the expense of count rate. If the detector in Fig. 5.1 were $120 \mathrm{~cm}$ from the drum edge, the ratio of count rates between position 2 and position 1 would be $(150 / 120)^{2}=1.56$, but the overall count rate would

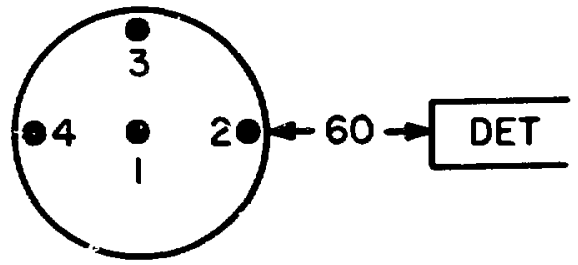

$$
\begin{array}{lll}
\mathbf{r}_{1}=90 \mathrm{~cm} & C R_{1} / C R_{1}(9 \mathrm{C} / 90)^{2} & 1 \\
\mathbf{r}_{2}=60 & C R_{2} / C R_{1}(90 / 60)^{2} & 2.25 \\
r_{3}=95 & C R_{3} / C R_{1}-(90 / 95)^{2} & 0.90 \\
r_{4}=120 & C R_{4} / C R_{1}=(90 / 120)^{2}=0.56
\end{array}
$$

Fig. 5.1.

Illustration of count rate variation with position in 55-gal drum. 
have dropped to nearly one third that at $60 \mathrm{~cm}$. A better procedure is to rotate the sample. Consider the following diagram.

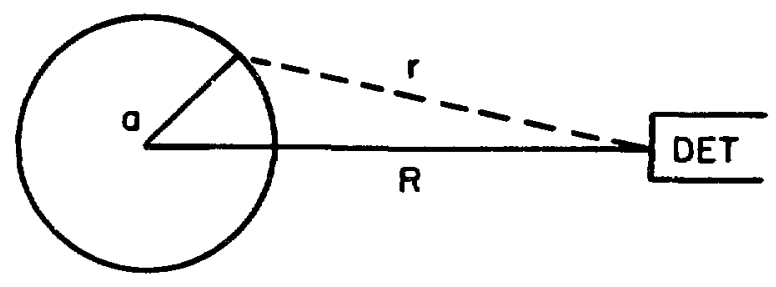

The ratio of the average response of a source rotating on the radius $(a)$ to the response at the center is

$$
\frac{C R(a)}{C R(0)}=\frac{1}{1-(a / R)^{2}} .
$$

Toble 5.1 lists this function for severcl values of $a / R$. By ratating the sample, the maximum count rate variation in Fig. 5.1, due to position, is reduced from 2.25 to 1.125 with no loss in overall count rate. Therefore, whenever possible, the sample should be rotated to minimize the potential error caused by nonuniform distributions of material within the sample. Rotation minimizes the effect of radial variations. If the sample is taller than it is wide, the vertical variation must be considered also. This is illustrated in Fig. 5.2 where $L=1 / 2$ height of container and $\mathbf{n} \cdot \mathrm{L}=$ distance from detector to center of container. The maximum variation is reduced to $10 \%$ with a sample-to-detector distance of $3 \mathrm{~L}$.

In general, the choice of sample-to-detector distance is a compronise between minimizing the response variation and maintaining an adequate count sate. A general guideline can be given as follows. The maximum count rate variation with position is less than $\pm 10 \%$, if the distance between the
TABLE 5.1

\section{THE EFFECT OF SAMPLE ROTATION ON COUNT RATE VARIATION}

\begin{tabular}{|c|c|c|}
\hline$\alpha / \mathbf{R}$ & $\begin{array}{c}\text { CR(a)/CR(0) } \\
\text { Rotating } \\
\end{array}$ & $\begin{array}{c}\text { CR(R-a)/CR(R) } \\
\text { Not Rotating }\end{array}$ \\
\hline $1 / 2$ & 1.33 & 4 \\
\hline $1 / 3^{a}$ & 1.125 & 2.25 \\
\hline $1 / 4$ & 1.067 & 1.78 \\
\hline $1 / 5$ & 1.042 & 1.56 \\
\hline $1 / 6$ & 1.029 & 1.44 \\
\hline $1 / 7$ & 1.021 & 1.36 \\
\hline
\end{tabular}

aThis is the case illustrated in Fig. 5.1.

center of the sample and the detector is equal to or greater than three times the larger of the dimensions $a$ or $L$ (radius or $1 / 2$ height) and if the sample is rotated.

$$
\begin{aligned}
& \frac{\Delta \mathrm{CR}}{\mathrm{CR}} \leq 10 \% \text { if } \mathrm{R} \geq 3 \mathrm{a} \text { or } 3 \mathrm{~L}, \text { whichever is } \\
& \text { larger, where a radius of sample and } \mathrm{L} \\
& =\frac{1}{2} \text { height and the sample is rotated. This } \\
& \text { applies to } 1 / \mathrm{r}^{2} \text { variations only. }
\end{aligned}
$$

If the sample cannot be rotated, it should at least be counted in two orientations $180^{\circ}$ apart. Unually there will be little need to increase the sample-to-detector distance beyond this beccuse of considerations of sample attenuation which will usually be the largest source of count rate variation. A source at the center of the sample will experience a larger attenuation than will a similar source near the edge. This nffect cannot be minimized by increaning the sample-todetector distance. In most samples it will be the domi-
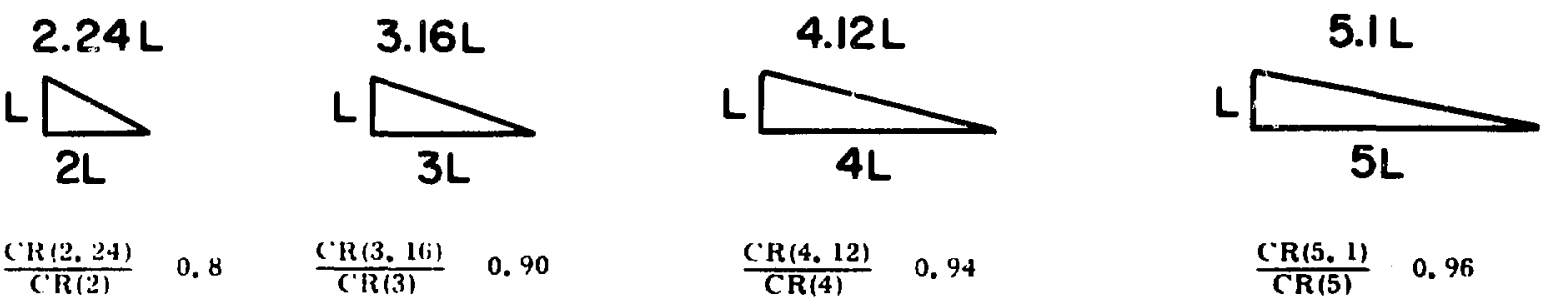

$\frac{(R ! 2.24)}{(R(2)} \quad 0.8 \quad \frac{(R(3,1(i)}{C(R(3)} \quad 0.90$

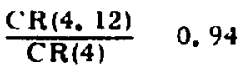

$\frac{C R(5,1)}{C R(5)} \cdot 0.96$

Fig. 5.2.

Maximum vertical count rate variation as function of sample-to-detestor distance. 
nant effect, so sample-to-detector distances larger than indicated by the above discussion and Eq. (5.4) are usually of little value.

\subsection{Gamma-Ray Attenuation}

Figure 5.3 is a graph of mass attenuation coefficient vs energy for a selected range of elements. It contains nearly all the qualitative attenuation information necessary for performing gamma-ray assay. Several important features should be noted. Between 1 and $3 \mathrm{MeV}$, the mass attenuation coefficients of all elements are equal within $~ \pm 20 \%$; the average value is about $0.05 \mathrm{~cm}^{2} / \mathrm{g}$. In this region, Compton scattering is the dominant process. If nature had equipped the isotopes of interest with an intense gamma ray in this range, gamma assay would be much easier. Unfortunately only ${ }^{238} U$ is so equipped. At lower energies the coefficients of the high-Z elements of interest become much larger than those of the lower $\mathrm{Z}$ materials, reaching values about 20 times higher near the K-absorption edge of uranium. These large differences make quantitative

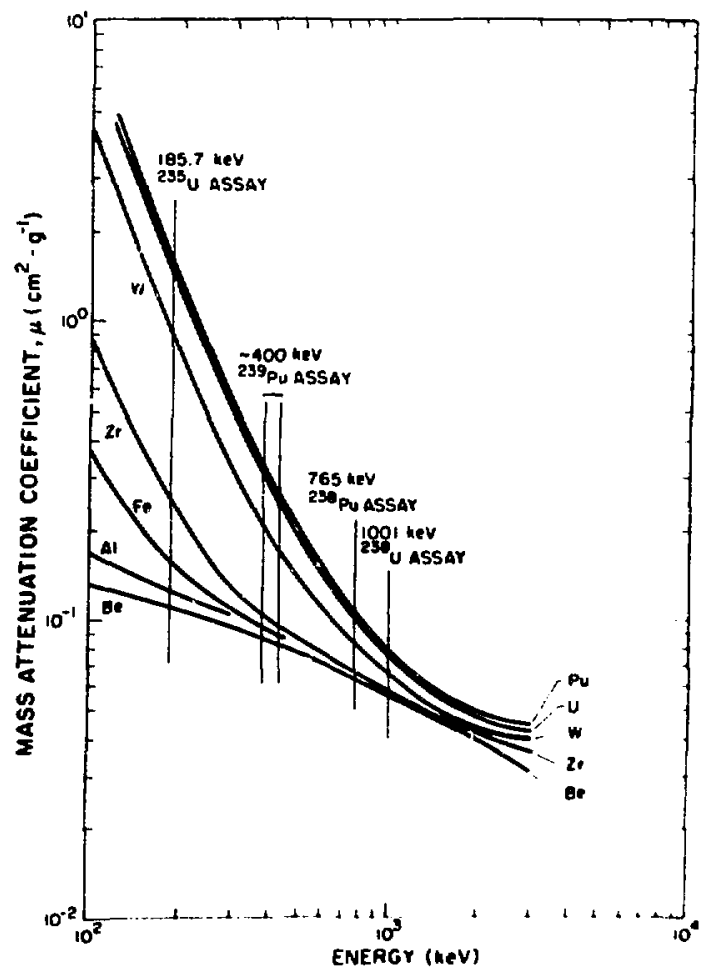

Fig. 5.3.

Mass attenuation coefficiom is energy for selected clenenss. The energies of several imporsans assay lines are indicated on the graph. assay by low-energy gamma rays difficult or even impossible. The mass absorption coefficients of uranium and plutonium are nearly sis times larger at $186 \mathrm{keV}$ than at $414 \mathrm{keV}$. This means the gamma assay of ${ }^{235} \mathrm{U}$ is subject to a greater potential error than the assay of ${ }^{239} \mathrm{Pu}$ due to the higher selfabsorption of the 186-keV gamma ray. The region between 80 and $120 \mathrm{keV}$ is usually not useful for assay measurements because of the $K \times$ ruys of uranium and plutonium. Below $80 \mathrm{keV}$ most attenuation ccefficients increase rapidl $y$, making attenuation problems unmanageably severe for most cases. Nearly all uranium and plutonium assay is done with gamma rays between 100 and $1000 \mathrm{keV}$.

\subsection{Attenuation Corrections}

The correcion for sample attenuation is probably the most important factor in gamma-ray assay. For this text the attenuation correction factor (CF) is defined by the following expression.

$$
C F=\frac{C R(\mu=0, \text { no attenuation })}{C R \text { (actual observed count rate) }}
$$

This expression is symbolic. CF cannot be computed from Eq. (5.5) because $\mathrm{CR}(\mu=0)$ cannot be measured directly. The product $\mathrm{CR} \cdot \mathrm{CF}$ [see Eq. (5.1)] is sometimes called the "corrected count," that is, the count rate which would be measured in the absence of attenuation. As defined, the correction factor has a minimum value of one. Experience has shown that the maximum value that can be determined with reasonable accuracy $( \pm 5 \%)$ is about tive. It should be empharized that $\mathbf{C F}=\mathbf{5}$ is a large corzection implying that only $20 \%$ of the gamma rays of interest escape from the sample. Large values of CF imply kigh potential for orror. Sample nonuniformities become more troublesome as CF increasen.

The bavic aseumption for all gamma asady is that the mixure' of uranium or phutonium and matrix maserial (everything other than uranium or plutonium in the samplo) is reusonably uniform, and the uranium or plulonium particles are smail enough to ignore self-attenuation within the omitting particles. It is difficult to define "reasonably uniform," but some rough guidelives can be discuwed. If the individual particles of uranium or plutonium have rignificant self-attenuation, the andy results will be low unlees opecial procedures are used to correct for this. (These procedures are beyond the scope of this text; 
they are also imperfect and impractical for moat portable instrumantation.) The self-attenuation of the individual particles can be extimated from the following formula.

$$
\begin{aligned}
& C F=\frac{\mu x}{1-e^{-\mu x}} \\
& C F \approx 1+\mu x / 2 \quad \text { if } \mu x \leq 1, \\
& C F \approx \mu x \quad \text { if } \mu x \geq 3,
\end{aligned}
$$

where

$$
\begin{aligned}
& \mu=\text { linear aftenuation coefficient of emiting } \\
& \text { material, } \\
& x=\text { mean linear dimension of particle and } \\
& \text { the approximations }(a, b) \text { are good to } 5 \% \text {. }
\end{aligned}
$$

The actual particles are irregular shapes and their size is not usually woll known, $s 0$ it is difficult to compute the self-attenuation exactly. This formula should only be ueed to extimate the order of magnitude of the particle self-attonuation. Figure 5.4 illuntrates the variation of self-attenuation with particle vire for uranium and plutonium. Smull particles can have

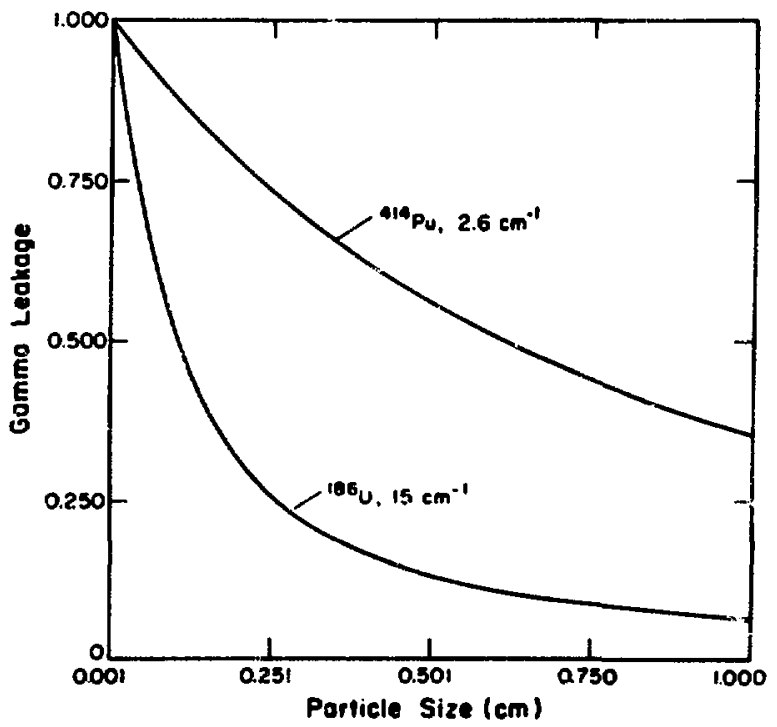

Fig. 5.4.

Self-attenuation vs particle size. significant self-attenuation, particularly uranium. A $130-\mu$ particle of uranium oxide will abworb $10 \%$ of the 186.keV gamma rays emitted by ${ }^{235} \mathrm{U}$ within the particle. Larger particles, wuch as fuel polletw, are ovon wores. A $1 . \mathrm{cm}$ pellot of $\mathrm{UO}_{2}$ requires a correction factor of about $15\left[\mu x \approx\left(1.5 \mathrm{~cm}^{2} / \mathrm{g}\right)(10\right.$ $\left.\mathrm{g} / \mathrm{cm}^{3}\right)(1 \mathrm{~cm})=15$ ] for the $186-\mathrm{keV}$ gamma ray. $A$ similar plutonium recycle pollet would require $\alpha$ correction factor of about 2.5 for the 414-keV gamma ray. If auch pellots ware in a contriner of low-density combutible waste (rags, gloves, Kimwipes, otc.), they would not meet the requirement of reasonable uniformity. HTGR-coated particles come close to meeting the requiremont, but ancy renult will still be $5.10 \%$ low if corrections are not made for particle size. Pure powdere $\left(\mathrm{PuO}_{2}, \mathrm{UO}_{2}, \mathrm{U}_{3} \mathrm{O}_{\mathrm{g}}\right.$, etc.) do meet the requirement as do certain well-mired powder corap materials such as most incinerator ash. Small quantities of powder mixed with ccnbustibles may meet the requirement if the powder is distributed uniforialy in the matrix and is not in lumps.

The above discussion illustrates some of the basic problems of gamma-ray assay. There are some techniques that allow less stringent uniformity conditions, but these are beyond the scope of this text and the capability of portable instrumentation. To perform gamma-ray assay with any assurance of accuracy, the assayist must know that the samples ineet the basic assumption of uniformity. In favorable cases, accuracies of $\pm 5 \%$ (one sigma) are readily obtainable; however, for samples that depart far from uniformity, measurements can be low by a factor of two or more. The remainder of this chapter will discuss common ways of computing the attenuation correction factor and will give several examples of

\begin{tabular}{|c|c|c|}
\hline CF & $\frac{\mu D}{1-e^{-\mu D}}$ & slab \\
\hline$C F$ & $\frac{\left.n / 4_{\mu} \mid\right)}{1-e^{-n / 4 \mu \eta}}$ & cylinder \\
\hline$C F$ & $e^{+\mu L}$ & alssorber \\
\hline
\end{tabular}
specific assay problems.

\section{4.a. Correction Factor Expresuione}

Equations (5.7) list several common exprescions for the correction factor. 


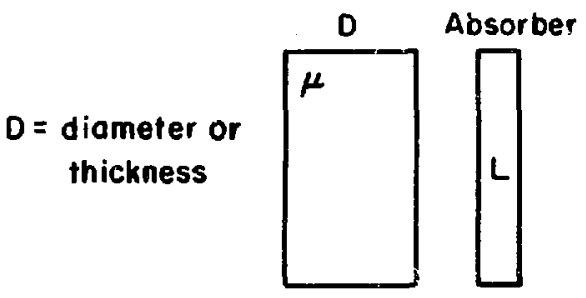

DET

The slab formula [Eq. (5.7a)] is an exact expression $f(x)$ the case where the sample-to-detector distance is very large compared with the dimensions of the source and the detector (this is sometimes called the far-field approximation). It is used for rectangular sarnples (plates, boxes, air filters, etc.) viewed parailel to a side (usually through the thin dimension). The expression works well even for fairly small sample-to-detecior distances (one or two times the sample thickness). Equation (5.7b) is a nearly exact expression for cylindrical samples. It has the same form as Eq. (5.7a) with $\mu \mathrm{D}$ replaced by $\pi / 4 \mu \mathrm{D}(\pi / 4$ $=0.785$ ). This expression works well even when the detector is only 1 diam from the edge of the sample. Both Eq. (5.7a) and Eq. (5.7b) are plotted in Fig. 5.5. Equation (5.7c) is merely the fundamental law of gamma attenuation and is used for absorbers placed between the sample and the detector. This expression would be applied to the walls of the sample container. For this case, the total correction factor would be the product of Eq. (5.7a) or Eq. (5.7b) times Eq. (5.7c).

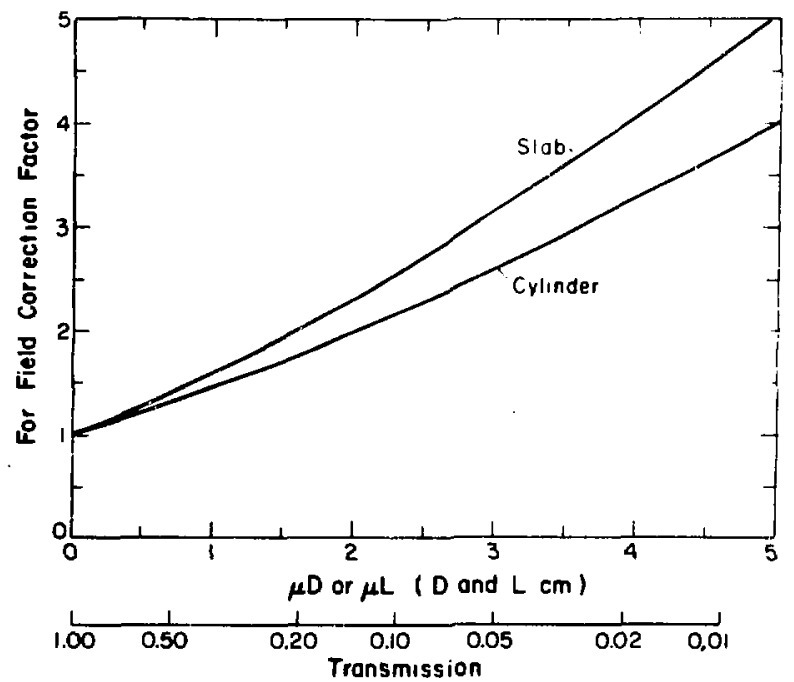

Fig. 5.5. Alremantion correction factor is $\mu D, T$ for slab and crlinder.
In general, these expressions are approximate but accurate, particularly for ues with high-resolution detectors. For NaI they usually overestimate $\mathrm{CF}$ due to the effects of amall-angle scattsre in the sample, as discussed in Chap. 1. In mang saves, $\mu \mathrm{D}$ can be determined by an external cource transmintion measurement as described in the next section. At other times (for equipment boldup and large wasto containers) $\mu \mathrm{D}$ is estimated from knowledge of the sample and CF is computed directly from the appropriate expression above.

\section{4.b. Transmission-Corrected Gamma-Ray Assay}

Consider the situation pictured in Fig. 5.6. The sample is placed between the detector and an external gamma-ray source. $I_{0}$ is the mearured intensity of the source with no sample, and I is the intensity with the sample in place. The transmission, $T$, is defined as

$$
T=I / I_{0}
$$

that is, the traction of gomma rays from the source which penetrates the sample with no change in energy. From the fundamental attenuation relationship [Eq. (1.5)],

$$
\mathbf{T}=\mathbf{e}^{-\mu D} \text {. }
$$

The correction factor equations [Eqs. (5.7)] can be rewritten in terms of $\mathrm{T}$.

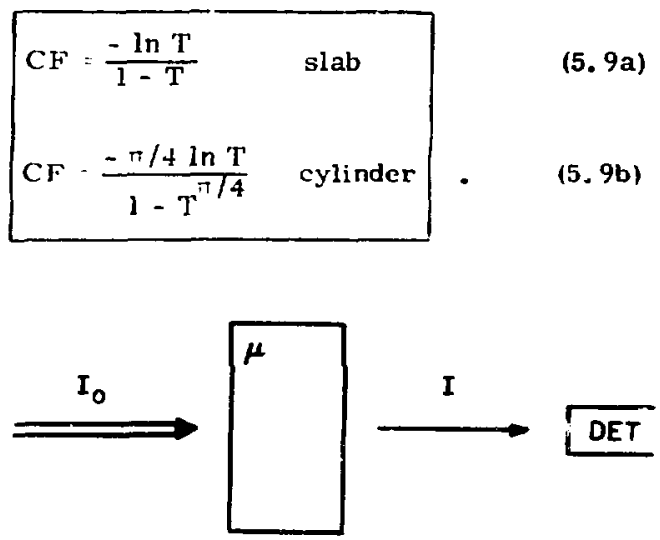

Fig. 5.6.

Diagram of a transmission measuremen. 
Figure 5.5 skows a plot of CF vs T. For T greater than $0.2, C F$ is less than 2.0 and doesn't change rapidly. This is a favorable range for assay work. For $T$ less than 0.2, CF rises rapidly and the possibility of error increases. Measured transmissions become less accurate when $\mathrm{T}$ is less than $10 \%$. For careful work with high-resolution detectors, transmissions as low as $\sim 0.5 \%$ (CF $\sim 5.3$ ) can be measured with confidence. Caution is advised when measuring transmissions below $10 \%$ with NaI.

Table 5.2 lists some of the cummon transmission sources. Equations (5.9a) and (5.9b) assume that the transmission is measured at the same energy as the asscy gamma ray. As indicated in Table 5.2, transmission and assay energies may be different, in which case a correction must be applied for the difference in attenuation between the two energies. This correction usually involves some knowledge of the composition of the sample. The relation between the transmissions at the two energies is given by

$$
\mathrm{T}_{\mathrm{a}} \mathrm{T}_{\mathrm{t}}^{\alpha}
$$

where

$$
\begin{aligned}
& \text { a refers to assay energy, } \\
& t \text { refers to transmission energy, and } \\
& \alpha=\mu_{a} / \mu_{t} .
\end{aligned}
$$

The measured transmission is raised to the $\mu_{a} / \mu_{t}$ powrer before substitution into Erģ. (5.9). Consider the assay of ${ }^{239} \mathrm{Pu}(414 \mathrm{keV})$ contaminated incinerator ash using ${ }^{137} \mathrm{Cs}(662 \mathrm{keV})$ as a transmission source. As in Chap. l, this mixture can be treated as two components, one having the attenuation properties of oxygen and the other having those of plutonium. Equation (1.8) is then used to calculate the composite attenuation. Table $5.3 \mathrm{ij}$ lustrntes the change in $\alpha\left(\mu_{\alpha} / \mu_{1}\right)$ with the plutonium weight fraction. Most incinerator ash will be less than $10 \%$ plutonium by weight, so $\alpha=1.27$ might be picked as an average value for the measurersents. If a wider range of weight fractions is encountered, it may be necessary to perform an iteration (that is, pick a trial $F_{\mathrm{Pu}}$, calculate the mass of plutonium, calculate $\boldsymbol{F}_{\mathbf{P u}}$ from this value and the sample net weight, recompute the mass of plutonium, etc.).

The choice of a transmission source is frequently limited by the equipment and sources available. Uranium or plutonium is usually available, and neither of them requires any energy correction as above. There are, however, three bad aspects of their use: they require a double measurement (with and without source) to compensate for the activity in the sample; at low transmissions this requires the subtraction of two large and nearly equal numbers, the result of which usually has $\alpha$ low statistical precision; and, finally, the high self-attenuations of uranium and plutonium make it difficult to get high-intensity sources. Sources such as ${ }^{169} \mathrm{Yb}$ and ${ }^{75}$ Se are usually chosen for use with high-resolution detectors. These are near enough to the assay energy to consider $\alpha=1$. NaI requires the use of uranium or plutonium or sources such as ${ }^{22} \mathrm{Na}$ and ${ }^{137} \mathrm{Cs}$ which are of sufficiently different energy as to reduce the in-

\begin{tabular}{|c|c|c|c|}
\hline $\begin{array}{c}\text { Assay } \\
\text { Isotope }\end{array}$ & $\begin{array}{c}\text { Assay } \\
\text { Energy } \\
\text { (keV) } \\
\end{array}$ & $\begin{array}{c}\begin{array}{c}\text { Transmission } \\
\text { Source }\end{array} \\
\end{array}$ & $\begin{array}{c}\text { Asscry } \\
\text { Energy } \\
\text { (keV) }\end{array}$ \\
\hline${ }^{235} \mathrm{U}$ & 186 & $\left\{\begin{array}{l}169 \mathrm{Yb} \\
235 \mathrm{U}\end{array}\right.$ & $\begin{array}{c}177,198 \\
186\end{array}$ \\
\hline${ }^{238} \mathrm{U}$ & 1001 & $\left\{\begin{array}{l}137 \mathrm{Cs} \\
{ }_{54} \mathrm{Mn} \\
22 \mathrm{Na}\end{array}\right.$ & $\begin{array}{r}662 \\
834 \\
1275\end{array}$ \\
\hline${ }^{239} \mathbf{P u}_{\mathbf{u}}$ & 414 & $\left\{\begin{array}{l}75 \mathrm{Se} \\
22 \mathrm{Na} \\
137 \mathrm{Cs} \\
239 \mathrm{Pu}\end{array}\right.$ & $\begin{array}{l}401 \\
511 \\
662 \\
414\end{array}$ \\
\hline
\end{tabular}
terference with the plutonium gamma rajs.

TABLE 5.2

COMMON TRANSMISSION SOURCES 
THE VARIATION IN $\mu(414) / \mu(662)$ WITH

PLUTONIUM WEIGHT FRACTION

\begin{tabular}{lc}
$\mathbf{F}_{\mathrm{P}_{u}}$ & $\alpha=\mu(414) / \mu(662)$ \\
\cline { 2 - 2 } 0 & 1.21 \\
0.1 & 1.33 \\
0.3 & 1.54 \\
0.5 & 1.71 \\
0.7 & 1.84 \\
0.9 & 1.95
\end{tabular}

If the sample is uniform, one transmission measurement will adequately define $\mu \mathrm{D}$. In more advanced procedures, the sample is scanned to measure $T$ as a function of position. ${ }^{5.1}$ This is usually not practical with portable equipment; however, measurements of transmission at more than one position may provide a crude check of sample uniformity and lend confidence to the assay.

\section{4.c. Other Attenuation Corrections}

Equation (5.11) gives an approximate correction factor which may be used for transmissions over $\sim 0.2$. This assumes that the average

$$
\begin{aligned}
& C F \approx 1 / \sqrt{T} \quad \text { slab } \quad \text { (5. 11a) } \\
& C F \approx U / \sqrt{T^{\pi / 4}} \text { cylinder }, \quad \text { (5.11b) }
\end{aligned}
$$

path length within the sample is one-half the slab thickness or one $\pi / 8$ th of the cylinder diameter. Table 5.4 gives a comparison of Eqs. (5.11) with the exact expressions of Eqg. (5.9). For T greater than 0.2 the approximate alab expression is less than $12 \%$ high and the cylinder expression is less than $7 \%$ high.

Cline ${ }^{5.2}$ has described a method for assaying piutonium waste by basing the atienuation correction on the differential absorption of two different plutonium gamma rays (usually $129 \mathrm{keV}$ and 414 keV). This procedure can also be combined with transmission measurements to provide information on possible lumps of emitting material. Due to the plutonium spectrum complerity, a bigh-resolution

$\begin{array}{ll}414 \mathrm{keV} & \frac{662 \mathrm{keV}}{\mu_{\mathrm{Pu}}=0.26 \mathrm{~cm}^{-2} / \mathrm{g}} \\ \mu_{0}=0.093 & \begin{array}{l}0.13 \\ 0.077\end{array}\end{array}$

detector is required to use this technique; therefore it will not be described here. The reader should consult the references. $5.2,5.3$

In some cases, useful estimates of CF can be made by having some knowledge of the sample weight and composition. If the snmple is fuil and if its weight and composition are known, the attenuation may often be calculated with sufficient accuracy so that no experimental measurement is required. Consider the example of a 55.gal drum of plutonium. contaminated combustible waste. The waste material must be well segregated (no lathe beds, ball mills, etc., mixed with the combustibles) and the plutonium concentration must be low so that it is a minor part of the total attenuation. Combustible waste will have attenuation properties similar to water.

TABLE 5.4

\section{COMPARISON OF APPROXIMATE AND EXACT EXPRESSIONS FOR CF}

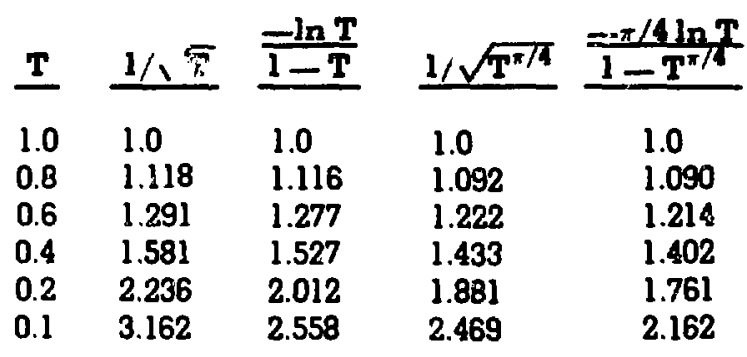


Known:

net weight $=32-\mathrm{kg}$; size : $56-\mathrm{cm}-$ diam by $89-\mathrm{cm}-\mathrm{high}$; walls $=0.1-\mathrm{cm}$; volume $=$ $0.22-m^{3}$.

Computations:

$$
\begin{aligned}
& \text { density }=\frac{32 \mathrm{~kg}}{0.22 \mathrm{~m}^{3}}=145 \mathrm{~kg} / \mathrm{m}^{3} \\
&=0.145 \mathrm{~g} / \mathrm{cm}^{3} \\
& \mathrm{H}_{2} \mathrm{O}: \mu(414)=0.104 \mathrm{~cm}^{2} / \mathrm{g} \\
& \mu_{\ell}=(0.104)(0.145\}=0.015 \mathrm{~cm}^{-1} \\
& D=56 \mathrm{~cm} \\
& \mu_{\ell} \mathrm{D} \quad(0.015)(56) \quad 0.84 .
\end{aligned}
$$

Using Eq. (5. 7b),

$$
\text { CF } \frac{(0.785)(0.84)}{1-e^{-(0.785)(0.84)}} \quad 1.37 \text {. }
$$

Correcting for absorption in the wall of the drum,

$$
\text { Fe: } \begin{aligned}
\mu(414) & =0.09 \mathrm{~cm}^{2} / \mathrm{g} \\
\mu_{l}= & (0.09)\left(7.9 \mathrm{~g} / \mathrm{cm}^{3}\right)=0.71 \mathrm{~cm}^{-1} \\
\mu_{l} J= & (0.71)(0.1 \mathrm{~cm})=0.071 .
\end{aligned}
$$

Using Eq. (5. 7c),

$$
C F=e^{0.071}-1.07 \text {, }
$$

So that the total correction factor is

$$
C F-(1.07)(1.37) \quad 1.47 \text {. }
$$

Consider the effect of $100 \mathrm{~g}$ of plutonium distributed uniformly in the drum.

$$
\begin{aligned}
\text { Pu density } & =\frac{0.1 \mathrm{~kg}}{0.22 \mathrm{~m}^{3}}=0.45 \mathrm{~kg} / \mathrm{m}^{3} \\
& =4.5 \times 10^{-4} \mathrm{~g} / \mathrm{cm}^{3}
\end{aligned}
$$

$$
\mathrm{Pu}: \mu(414)=0.26 \mathrm{~cm}^{2} / \mathrm{g}
$$

$$
\mathrm{He}_{l}(0.26)\left(4.5 \times 10^{-4}\right)
$$

$$
1.2 \times 10^{-4} \mathrm{~cm}^{-1}
$$

$$
\mu_{l} \mathrm{D}\left(1.2 \times 10^{-4}\right)(56) \quad 0.7 \times 10^{-3} .
$$

Using Eq. (5, 7b),

$$
\text { C. } 1.003 .
$$

This says that $100 \mathrm{~g}$ of plutonium will have $\alpha$ negligible effect on the attenuation in the drum if it is distributed so that there are no self-attenuating lumps.

The important factor in this attenuation correction is that the samples must be filled or the fill volume must be known. The attenuation correction is based on the density of the sample. Consider a group of samples filled to different heighto with material of approximately constant density. Because the density is constant, the correction factor should be constant. However, if only the weight is known and the samples are assumed to be full, different correction factors will be applied to each sample.

The final procedure to be discussed involves the use of standards to cover the range of mnterial to be measured. In this care, no explicit computation or measurement is made to correct for attenuation. The unknowns are assumed to have the same attenuation properties as do the standards. This procedure is acceptable where there is a class of samples very vearly identical in size, shape, and composition, varying only in concentration of uranium or 
plutonium. In such cases, the attenuation of the matrix will be nearly constant from one cample to another and the obeerved count rate will be uniquely related to the tissionable material concentration. This selationship can be determined by preparing a series of stcindards covering the expected concentration range and counting them in the same geometry as the unknowns. The resulting calibration curve may be somewhat nonlinear if the uranium or plutonium concentration is high enough so that it begins to contribute to the attenuation in the standard. This procedure is the simplest to use, and, where applicable, can yield acceptable results. At present, it is probably the most widely used gamma assay procedure. ${ }^{5.4}$ For example, this would be used for product control or quality assurance where deviations from a mean (the standard) are to be measured. However, the standards procedure is susceptible to arzor and must be applied with caution. It should only be used on very well-controlled material because there is no check to prove that the unknowns actually do resemble the standards. The standar to must have the same matrix attenuation as the unknowns, otherwise there will be a constant bias on all measurements. Some users have constructed standards by placing small amounts of uranium or plutonium in vials and distributing these vials throughout a standard matrix. This can be a particularly poor procedure because the probability of self-attenuation in lumps or uranium or plutonium is very high, as discussed earlier. Consider $0.5 \mathrm{~g}$ of uremitim es $\mathrm{UO}_{2}$ (bulk $\mathrm{U}$ density $=2 \mathrm{~g} / \mathrm{cm}^{3}$, ignore attenuation in $\mathrm{O}$ ).

If contained in a cube, the dimenrion of that cube is

$$
\begin{aligned}
\left(0.5 \mathrm{~g} / 2 \mathrm{~g} / \mathrm{cm}^{3}\right)^{\frac{3}{3}} & =0.63 \mathrm{~cm} \\
U: \mu(186) & =1.5 \mathrm{~cm}^{2} / \mathrm{g} .
\end{aligned}
$$

Uaing Eq. (5.6),

$$
\begin{aligned}
& \mu x=\left(1.5 \mathrm{~cm}^{2} / g\right)\left(2 \mathrm{~g} / \mathrm{cm}^{3}\right)(0.63 \mathrm{~cm})=1.9 \\
& C F=\frac{1.9}{1-e^{-1.9}}=2.2 .
\end{aligned}
$$

That in, leas than half of the 186-keV gamma raye will get out of this volume unattenuated. For $\mathrm{PuO}_{2}$ $\mu(414)=0.26 \mathrm{~cm}^{2} / \mathrm{g}$, wo this becomes

$$
\begin{aligned}
& \mu x=(0.26)(2)(0.63)=0.33 \\
& C F=1.17 .
\end{aligned}
$$

Though the self-attenuation is smaller, it still represents a nonnegligible error that will whow up as a bias (aesay will bo high) on all mocurarenents. This problem must be considered when contructing standards for any gamma amay procedure; distributed vials of fissionable material are bad. news. Other factors to consider include settlement and compaction in the standards (and the unknowns) and soparation of the fissionable oxide from the matrix. The bulk density of most powders can be changed over a considerable range by agitation and vibration. These procedures can aleo cause the timionable oxide to settle out of the matrix if there are gromly different particle sire distributions. All of this indicater that care must be used when applying a gamma asay procedure that relies on standards only.

\subsection{Example 1: Sraall Cane of Plutonium In- cinerator Ash}

This problem is the verification of the plutonium content of small cans of incinerator wh. The cans are $30-\mathrm{cm}$ high by $15-\mathrm{cm}$ o.d. and are doubly bagged. The contents have been blended, therefore it is safe to assume that the mixture of plutonium and matrix is uniform. Up to $100 \mathrm{~g}$ of plutonium will be encountered, all of contant isoiopic, 85\% ${ }^{23 \%} \mathrm{Pu}$. The equipment cruilable is a 5-by 5-cm Nal detector writh a portable electronice package containing two single-channel analyzers. There is aleo a simple rotator and a $400-\mu \mathrm{Ci}{ }^{22} \mathrm{Na}$ source for tranmiscion measurement. There is a single 50-g plutonium standard available in the same type of container. The alope of the Compton background around $400-600$ koV can be aroumed lovel so that $k=1$ in Eq. (4.3a). One hundred groms of plutonium is lew than $2 \%$ plutonium by weight, and the plutonium attenuation is mall ( $<10 \%)$. The matrix is asoumed to attenuate like oxygen, $\mu(414) / \mu(511) \approx 1.1$. The assay procedure is outlined bolow for correful study. 


\section{A. Setup}

1. Sample-to-detector distance $=45 \mathrm{~cm}$ (use 5.2 , $1 / 2$ height $=15 \mathrm{~cm}$ )

2. Detecior to transmission source $=75 \mathrm{~cm}$. Position the source to look through the center of the sample.

3. When the energy calibration is determined, set the windows as follows.

\section{P: $375.450 \mathrm{keV} \quad 414-\mathrm{keV}$ peak from ${ }^{239} \mathrm{Pu}$.}

B,T: $460.560 \mathrm{keV} \quad 414 \cdot \mathrm{keV}$ background window and $511-k e V$ peak window. The background window for the transmission gamma ray is omitted here to eliminate changing SCA threshold between measurements.

4. Put a cadmium absorber $(0.08-\mathrm{cm}$ to $0.15-\mathrm{cm})$ over the face of the detector to reduce the intensity of the $60-\mathrm{keV}$ gamma ray from ${ }^{241} \mathrm{Am}$.

\section{B. Measurement Procedure}

1. Using the high-energy window, measure the transmission source to determine the "straightthrough" count rate, $\mathrm{CR}_{0}$. Measure several times and take the average.

2. Calibrate the system by measuring the standard, then measlire the unknowns. Recheck the instrument calibration occasionally by repeating the standard measurement.

3. Cover the trarsmisaion source with lead brick (or remove the source). Place the sainple on the rotator and measure the activity in both windows, $\mathrm{CR}_{\mathrm{p}}$ and $\mathrm{CH}_{\mathrm{b}}$

4. Uncover the transmission source and measure the transmission throigh the sample (use the higher energy window) $\mathrm{CR}_{\text {r }}$

\section{Results and Sample Computation:}

1. All counts are $60 \mathrm{~s}$.

2. $\mathrm{CR}_{0}=252000$.

3. Calibration, using the $50-\mathrm{g}$ standard.

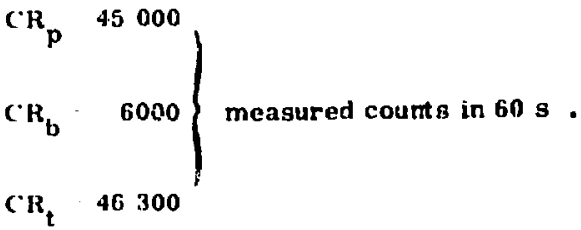

Using Eq. (5. 8),

$$
T_{t}=\frac{C R_{t}-C R_{b}}{C R_{0}}=\frac{46300-6000}{252000}=0.16 .
$$

Uaing Eq. (5. 10),

$$
T_{a}=(0.16)^{1.1}=0.133
$$

Using Eq. (5, 7b),

$$
\begin{aligned}
& C F_{s}=\frac{-(0.785) \ln (0.133)}{1-0.1330 .785}=1.99 \\
& C R_{s}=\frac{C R_{p}-C R_{b}}{60}=\frac{45000-6000}{60}=650 / \mathrm{s} .
\end{aligned}
$$

(It is not necessary to divide by the count time; this just keeps the numbers smaller.)

Using Eq. (5. 2),

$$
K=\frac{C R_{g} \cdot C F_{B}}{M_{g}}=\frac{650 \times 1.99}{50}=25.9 / g-8 \text {. }
$$

4. Uncertainty in calibration.

The standard is measured four more times yielding values of 25.5, 26.2, 25.8, and 26.1. The mean of these measurements is $25.9 / \mathrm{g}-\mathrm{s}$, and the standard deviction in the Imesull is $0.1 / \mathrm{g}$-s.

5. Measurement of unknown.

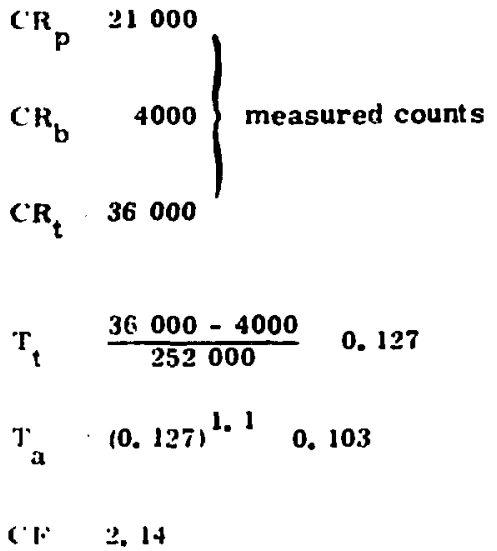




$$
\text { CR }=\frac{21000-4000}{60} 283 .
$$

Using Eq. (5. 1),

$$
M=\frac{C R \cdot C F}{K}=\frac{(283)(2.14)}{25.9}=23.4 \mathrm{~g} \mathrm{Pu} .
$$

6. Uncertainty in measuring the unknown.

A complete assay should include an estimate of the uncertainty in the measurement. This will be discussed more fully in Appendix B. For now, the formula below can be used to estimate the unceriainty in the measurement due to counting statistics. (This formula is approximate, and its derivation is not obvious.)

$$
\begin{aligned}
& \frac{\sigma M}{M} \approx \\
& \sqrt{\frac{C R_{p}+C R_{b}}{\left(C R_{p}-C R_{b}\right)^{2}}+(0.25)\left(\frac{C R_{t}+C R_{b}}{\left(C R_{t}-C R_{b}\right)^{2}}\right)+\left(\frac{\sigma K}{K}\right)^{2}}
\end{aligned}
$$

Equation (5.12) gives $\sigma \mathrm{M} / \mathrm{M} \approx 0.01$. It should be emphasized that this is the uncertainty due to coun. ting statistics only. The total uncertainis on a measurement such as this would be at lerint $10 \%$ itwo sigma).

\subsection{Example 2: Uranium-Contaminated Mir Filtere}

This problem involven measuring HEPA oir filtore (60. by 60 . by $30 . \mathrm{cm}$ ) for uranium sontent. The uranium is of constant enrichment, $93 \% 235 \mathrm{~J}$. The name detector and electronic equipment are avcilable $\boldsymbol{w}$ in the previoun example. Thore is no transmission source available, and the only standard is a andl foil of uranium motal: 2.5 . by 2.5. by 0.005 cm-thick, density $=19 \mathrm{~g} / \mathrm{cm}^{3}, 0.59-\mathrm{g}$ uranium $(93 \%$ 235 U). The filter woigh about $9.1 \mathrm{~kg}$ excluding the wood frame. The filter medium ahould attenuate similar to wator. Une $k=1.3$ for Eq. (4.3a).

\section{A. Sotup}

1. Sample-to-dotector distance $=90 \mathrm{~cm}$ (uve 5.2 , $1 / 2$ hoight $=30 \mathrm{~cm}$ ).

\section{Set the window as follown.}
P: $160 \cdot 210 \mathrm{keV}$
$186 \mathrm{keV}$ peak from ${ }^{235} \mathrm{U}$
B: $220 \cdot 270 \mathrm{keV}$
Background from 186.keV peak

3. Put a $0.08-\mathrm{cm}$ cadmium abuorber over the detector to reduce the intensity of uranium $x$ rays. Shield the detector with lead to reduce background radiation.

\section{B. Meanurement Proceduxe}

1. Mecrenre the foil etandard for calibration. Be sure to consider possible attenuation in the foil. The foil must be measured close to the detector $(30 \mathrm{~cm}$ or lesw) to get sufficient count rate. This necessitates applying a $1 / \mathrm{x}^{2}$ correction to the calibration.

2. Estinate the correction factor for the unknown filter.

3. Eeccuse of their thape, the filters should not be rotated, but should be counted on each side. Use the average of the two sides to compute the uranium cavay. If these two count differ greatly, the filter is not loaded unifornly. Another check on thin last point would involve holding the detector close to the filter and looking for hot spots. If these considerations indicate groes nonuniformities, a large uncertainty should be astigned to the meaturement.

4. Compute the uranium contont of the filter and estimate the uranium attenuation assuming a un. iform distribution. Sir filters hove been measured with $1 \mathrm{~kg}$, or more, of enriched uranium in a nonuniform distribution. For such as case, the uranium self-attenuation is considerable and the simple ccmputational procedure outlined here is not adequate. If lese than about $100 \mathrm{~g}$ are found, this procedure is probably accurate.

\section{Results and Sample Computations}

1. Culibration foil attenuation.

$$
\begin{aligned}
\mu(186) & 1.5 \mathrm{~cm}^{2} / \mathrm{g} . \\
\mu_{l} & =\left(1.5 \mathrm{~cm}^{2} / \mathrm{g}\right)\left(19 \mathrm{~g} / \mathrm{cm}^{2}\right) \\
& =28.5 \mathrm{~cm}^{-1}, \text { and }
\end{aligned}
$$




$$
\left.\mu D=.28 .5 \mathrm{~cm}^{-1}\right)(0.005 \mathrm{~cm})
$$

0.143 .

Using Eq. (5. 7a).

$$
\text { CF } \frac{0.143}{1-e^{-0.143}} \cdot 1.073 .
$$

2. Calibration. Mecrsure foil at $30 \mathrm{~cm}$. All count times $=60 \mathrm{~s}$.

$$
\begin{aligned}
& C R_{p}=3370, \\
& C R_{b}=600, \text { and } \\
& C R_{s}=\frac{3370-(1.3)(600)}{60}=43.2 / \mathrm{s} .
\end{aligned}
$$

Using Eq. (5, 2),

$$
\begin{aligned}
& \mathrm{K}=\frac{(43.2)(1.073)}{(0.59)(9)}=8.73 / \mathrm{g}-\mathrm{s} \\
& \text { (The } 1 / \mathrm{r}^{2} \text { correction for the dif- } \\
& \text { ferent positions is } \left.9 .(90 / 30)^{2} .\right)
\end{aligned}
$$

The standard is measured four more times yielding values of $8.47,8.98,8.76$, and 8.87 . The mean of these measurements is 8.76 and the standard deviation in the mean is 0.09 . Considering the assumptions made and the computed correction factor, this should be rounded off to two significant figures.

$$
K=8.8 \pm 0.1
$$

\section{Filter attenuation correction.}

$$
\begin{aligned}
\text { volume } & =(60 \times 60 \times 30) \\
& =1.08 \times 10^{5} \mathrm{~cm}^{3} \\
\text { density } & =(9100 \mathrm{~g}) /\left(1.08 \times 10^{5} \mathrm{~cm}^{3} ;\right. \\
& =0.084 \mathrm{~g} / \mathrm{cm}^{3}, \text { and }
\end{aligned}
$$

$$
\text { for water: } \mu(186)-c .14 \mathrm{~cm}^{2} / E \text {. }
$$

Linear attenuation coefficient of filter medium:

$$
\begin{aligned}
& \mu_{\ell}=(0.14)(0.084) 0.012 \mathrm{~cm}^{-1} \\
& \mu_{\ell} D=\left(0.012 \mathrm{~cm}^{-1}\right)(30 \mathrm{~cm}) 0.36 .
\end{aligned}
$$

Using Eq. (5, 7a),

$$
C F=\frac{0.36}{1-e^{-0.36}}=1.19 .
$$

(This correction factor considers only the filter medium. There is no correction for self-absorption in the uraniun. )

4. Measurement of unknown filter.

$$
\begin{aligned}
& C R_{p} 21600,21000, \\
& C R_{b} 1200,1300, \\
& C 11 \quad 20040,19310, \\
& C R \quad \frac{20040+14310}{(2)(f i 0)} 328 / 5 .
\end{aligned}
$$

Using Vq. (5. 1).

$$
M \frac{(328)(1.19)}{8.8} \quad 44 \mathrm{~g} \text { uraniım. }
$$

There is a $4 \%$ difference between the couni rates from the two sides. The relative precirion of each count is about $0.7 \%(1 \sqrt{ } 20000)$, therefore there is a alight difference in uraniurn activity between the two sides. This difference is amall and should not affect the assay cedversely.

5. Estimate of uranium attenuation.

$$
\begin{aligned}
\text { uraniun denatry }= & (14 \mathrm{c}) /\left(2.08 \times 10^{5} \mathrm{~cm}^{3}\right) \\
& 4.1 \times 10^{-4} \mathrm{~g} / \mathrm{cm}^{3} .
\end{aligned}
$$




$$
\begin{aligned}
& \mu(186) 1.5 \mathrm{~cm}^{2} / \mathrm{g} . \\
& \mu_{\ell} \cdot(1.5)\left(4.1 \times 10^{-4}\right) \\
& 6.1 \times 10^{-4}, \text { and } \\
& \mu D=\left(6.1 \times 10^{-4}\right)(30 \mathrm{~cm}) \\
&= 0.018 .
\end{aligned}
$$

Using Eq. (5. 7a),

$$
\mathbf{C F}=1.01 .
$$

The uranium attenuation is indeed small, and the ass umption to neglect if was justified. If the uranium content becomes much larger than this, its contribution to the attenuation must be considered. One kilogram of uranium uniformly distributed in the filter would require a correction factor of 1.22 (a total correction of $1.22 \times 1.19=1.45$ ). The assumption of $\alpha$ uniform distribution would probably not be justified for this case.

\subsection{Example 3: Measurement of Equipment Foldup}

The problem is to measure highly onriched $(93 \%$ 235U) uranium holdup in a low-velocity air duct used to carry machining residues away from various machines in a urcinium fabrication shop. The shop has been shut down, but a small amount of uranium residue is left on the walls of the duct. The equipment includes a $5.1 \mathrm{~cm}$-diam by $2.5 \mathrm{~cm}$-thick $\mathrm{NaI}$ detector in a lead shield and sollimater. The dintance $d$ from the detector face to the frort of the cylindrical colimator is adjutable from 0 to $10 \mathrm{~cm}$. $\mathrm{A} 0.08-\mathrm{cm}$ thick cadmium filter is toped to the detector face to attenuate low-energy $x$ rays. The electronics is a portable unit containing a high-voltage eupply, an amplifier, one SCA, a scxuler, and a timer, all mounted on a mall cart (Fiy. 5.7). Figure 5.7 shows an actual measurement in progress. An enriched 19 . g uranium foil (effective inass) is available for calibration.

The duct ( $41-\mathrm{cm}$-diam by $36-\mathrm{m}$-long) must be moasured in sections. Phyaical obstructions limit the ecrmple-to-detecior distance to about $1.4 \mathrm{~m}$ or more. It is neceseary to adjust the collinnator for a convenient

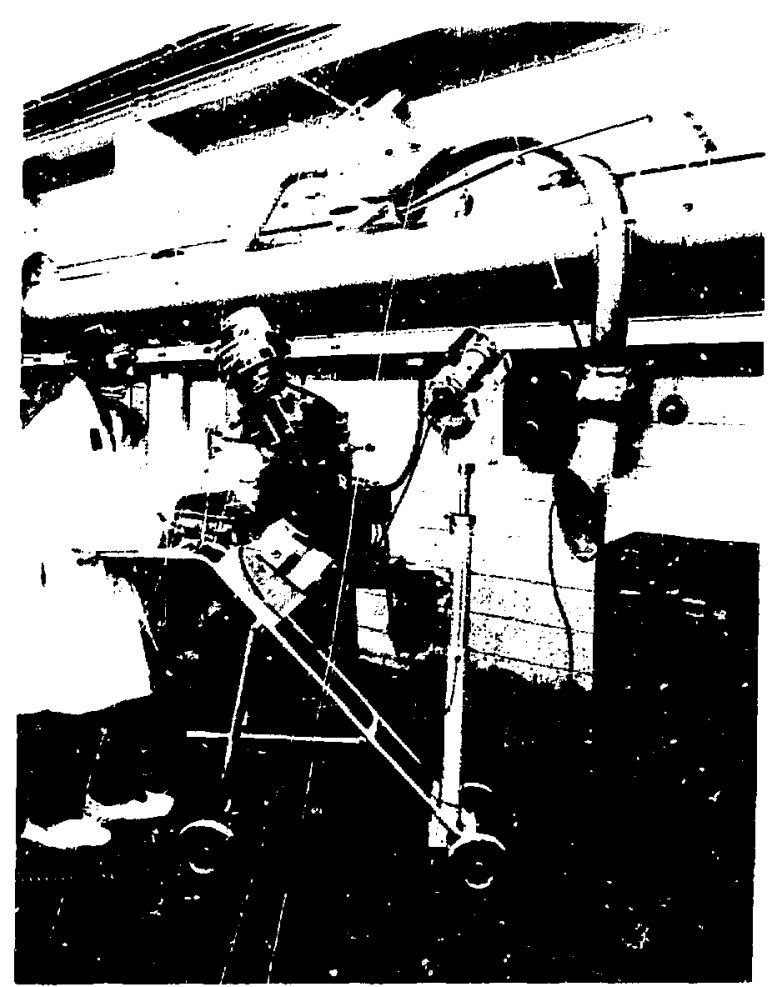

Fig. 5.7.

Measurement of uranium holdup in a duct using carl-moumled $\mathrm{NaI}$ and $\mathrm{S} . \mathrm{M} M-!$.

viewing cngle. The geometry of the situation is illustrated in Fig. 5.8. The detection efficiency $\&(R, r)$ is a function of $R_{,} r$. At a given $R$ it is maximum on the detectot centerline and decreases off the centerline. The distance $\mathbf{L}$ defines the effective extent of the collimator's view. Figure 5.9 shows a typical efficiency curve obtained by counting the calibration source at a fixed $R(1.4 \mathrm{~m})$ and varying $\mathrm{r}$. The collimator depth d is set at $6 \mathrm{~cm} . \mathrm{L} / 2$ is defined as the width of a step function of height $c(\mathrm{R}, 0)$, which has the same area as the actual efficiency curve (Fig. 5.9). This definition is equivalent to assuming that the efficiency is

$$
\begin{array}{rlrl}
\epsilon(R, r) & =\epsilon(R, 0) & \text { if } & -L / 2 \leq r \leq L / 2 \\
& =0 & \text { if } \quad|r|>L / 2 .
\end{array}
$$

This simplifies the calibration procedure because only a single measurement is required to determine $\epsilon(R, 0)$. As shown in Fig. 5.9, the efficiency drops to 


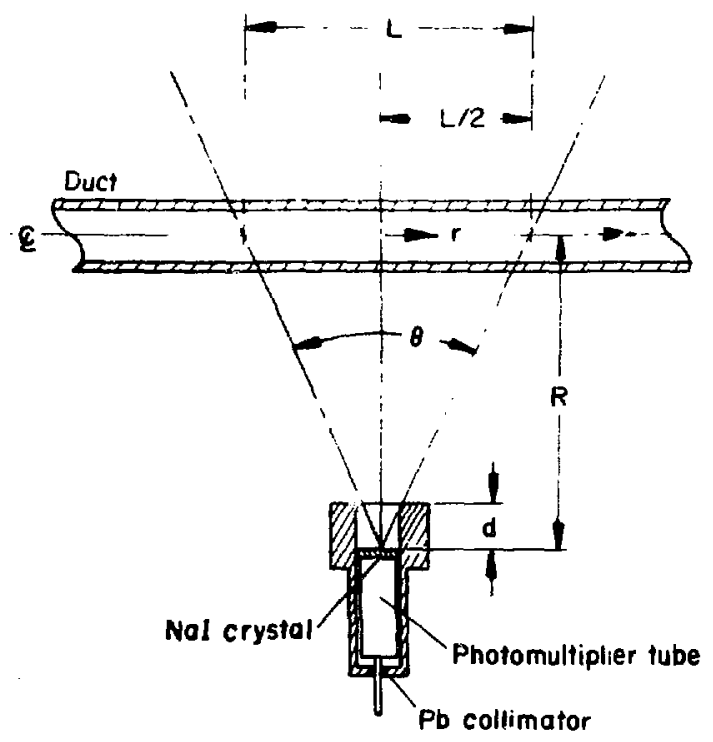

Fig. 5.8.

Diugram of duct measurement geometry.

$\sim 1 / 2 t(R, 0)$ at $L / 2$. This is a good rule of thumb. $L / 2$ is usually determined with sufficient accuracy by finding the point where the count rate from the calibration source drops to one-half the rate on the centerline.

The viewing angle, or collimation angle, $\theta$ can be defined as

$$
\theta=2 \tan ^{-1}\left(\frac{L(R)}{2 R}\right) .
$$

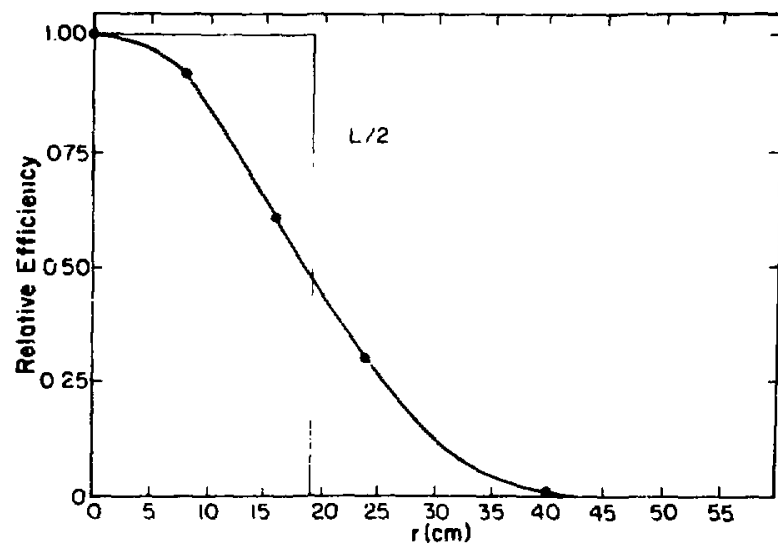

Fig. 5.9.

Relative detection efficiency vs lateral distance from detector centerline.
This is constant for $R \gg d$; $\theta$ is basically a function of $d$ only. Having determined $L$ for one distance $R$, it can be calculated for any other distance $R_{1}$ using

$$
L\left(R_{1}\right)=\frac{R_{1}}{R} L(R) .
$$

At the different spacing the detection efficiency changes by the inverse square of the distance.

$$
\epsilon\left(R_{1}, 0\right)=\left(\frac{R}{R_{1}}\right)^{2} \epsilon(R, 0) .
$$

Physical obstructions may prohibit making all measurements at a fixed distance so that Eqs. (5.15) and (5.16) are used to make the appropriate corrections for different sample-to-detector distances. When setting up to make holdup measurements on any type of equipment, the first step is to select the proper collimation. For the situation described here $(\mathrm{R}=1.4 \mathrm{~m}, \mathrm{~d}=6 \mathrm{~cm}), \mathrm{L} \approx \mathrm{lm}$, which is convenient for the duct measurement.

The next step is to calibrate the system. The desired sample-to-detector distance is $1.4 \mathrm{~m}$, as measured from the face of the detector to the center of the duct. Adjust the collimator to the desired depth, $d=6 \mathrm{~cm}$. Tape the calibration source to $a$ wall and aim the detector at the source. The separation must be $1.4 \mathrm{~m}$. The SCA is set 130 to $210 \mathrm{keV}$. Because this is highly enriched uranium, the Compton background from the source is small, therefore the upper background window is not necessary. It is necessary to subtract the ambient background in the peak window. Take several 60-s counts with the detector aimed at the source; these average 3226. Now aim the detector away from the source (e.g., at the floor) and make several 60-s counts to measure the ambient background. Be sure that the detector is not pointing at any suspected uranium holdup area. The average of these measuremenis is 120 . The calibration factor $\mathrm{k}$ is then

$$
k=\frac{3226-120}{19}=163 \text { count s/g }
$$

for $\alpha$ 60-8 count.

The final step is to evaluate the styryation. One method is to calculate the wall attenuation and assume that the uranium attenuation is mall. The 
wall thickness is $0.08 \mathrm{~cm}$ of steel $\left(\mu \approx 1.2 \mathrm{~cm}^{-1}\right)$. Using Eq. (5.7c), the appropriate correction factor is

$$
C F=e^{(1.2)(0.08)}=1.10 .
$$

All measured counts are multiplied by CF. After the uranium is mecrsured, it is possible to estimate the uranium attenuation by making reasonable assumptions about the distribution in the equipment. In some cases it may be reasonable to assume that the uranium is distributed in a uniform layer over the in. ner surface. This assumption allows computation of the layer thickness and the uranium attenuation correction. Suppose the mecisurement gives $200 \mathrm{~g}$ in a $\mathrm{l}$ $\mathrm{m}$ section. The thickness $\mathrm{x}$ of this layer is given by the masa divided by the surface area.

$$
x=\frac{200}{\pi(41 \mathrm{~cm})(100 \mathrm{~cm})}=0.015 \mathrm{~g} / \mathrm{cm}^{2}
$$

Using Eq. (5.7a), the appropriate correction factor would be $\left(\mu=1.5 \mathrm{~cm}^{2} / \mathrm{g}\right)$.

$$
C F=\frac{(1.5)(0.015)}{1-e^{-(1.5)(0.015)}}=1.01 \text {. }
$$

This is cextainly small and can be neglected. It may seem more reasonable to assume the material is a layer spread more or less uniformly over the bottom of the duct (assume that $1 / 8$ of the total surface area is covered). In this case, $x=0.12 \mathrm{~g} / \mathrm{cm}^{2}$, and $C F=$ 1.09. This is a larger correction and illustrates the biggest problem with equipment holdup measurements. The material distribution will uevally be uncertain or unknown and large errors can be in. troduced because of the uncertainty in the attenua. tion correction. If the $200 \mathrm{~g}$ is in one emall lump in the bottom of the duct, an even larger error will result if the usual uniform distribution is aseumed. In some cases careful transmission measurements may aid in evaluating the attenuation. However, a transmission measurement is only useful if the material approximater a uniform distribution. However, if the measurement shows less than $200 \mathrm{~g}$ in $1 \mathrm{~m}$, there is $\alpha$ reasonable assurance from the above estimates that the answer is not of by more than $20 \%$.

The actual measurements are then very straightforward. Make a 60-8 count every $1 \mathrm{~m}$ of the duct (A) and the Iocal background (B) (point detector away from duct). The uranium content of erich rection is computed from

$$
\begin{aligned}
\mathrm{gU} & =C F \cdot(A-B) / k=\frac{1.1}{163}(A-B) \\
& =6.7 \times 10^{-3}(A-B) .
\end{aligned}
$$

Every so often the calibration foil should be counted to see that the instrument hase not drifted. Some typical data are presented in Table 5.5, where the numbers give some confidence in the asenimption of small uranium attenuation because they are all con. siderably below $200 \mathrm{~g}$. Under favorable circumstances (i.e., small attenuation) as presented in this example, equipment holdup measurements can be accurate to $\sim 20 \%$. For equipment and uranium quantities where the attenuations are large, the associated measurement error will be large and probably will not be of a random nature. The gamma measurement of holdup usually will be biased low unless very conservative attenuation assumptions are made.

\subsection{Instrument Dead Time}

When a gamma ray interacts in a detector, there is a finite time before the counting system (detector and electronics) recovers and can accept another gamma.ray event. The time required for this recovery is called the "dead time," $t_{d}$. If the total count rate is high, the system may actually respond to only a fraction of the total gamma rays interacting in

\begin{tabular}{|c|c|c|c|}
\hline $\begin{array}{l}\text { Position } \\
\text { (m) }\end{array}$ & $\mathbf{A}$ & $\mathbf{B}$ & qU \\
\hline $\begin{array}{l}1 \\
2 \\
3 \\
4 \\
5\end{array}$ & $\begin{array}{l}3480 \\
7618 \\
4906 \\
9230 \\
5121\end{array}$ & $\begin{array}{l}110 \\
92 \\
77 \\
140 \\
123\end{array}$ & $\begin{array}{l}23 \\
50 \\
32 \\
61 \\
33\end{array}$ \\
\hline
\end{tabular}
the detector, that is, the actual counting period is shorter than the "real time" indicated by a clock.

TABLE 5.5

\section{URANIUM HOLDUP MEASUFEMENT}


The observed count must be corrected for the frac. tion of time the system is decd. If $R$ is the total count rate, this correction is given approximately by the following expression.

$$
C T=\frac{1}{I-R \cdot t_{d}} .
$$

For the systerns considered here, $t_{d}$ is the order of $2-5$

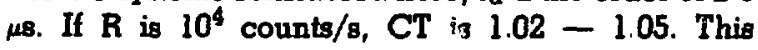
correction is small and usually can be neglected if the total count rate is below $10^{4}$ counts/s. It is advisable to operate portable instrumentation within this limit. It should be emphasized that the preceding discussion is a very simplified and incomplete treatment of instrument dead time; it is meant as a warning against high count rates.

\section{REFERENCES}

5.1. J. L. Parker, T. D. Reilly, J. E. Foley, R. B. Walton, and L. V. East, "Passive Assay-Innovations and Applications," Proceedings of the 12th Annual Meeting of the INMM, 1971, p. $514 \mathrm{ft}$. This paper discusses some of the scan techniques of gamma assay. There is a discussion of enrichment measurements and neutron counters which relates to other parts of this text.
5.2. J. E. Cline, "A Relatively Simple and Precise Technique for the Assay of Plutonium Waste," Aerojet Nuclear Company report ANCR-1055 (February 1972). This report describes the use of differential attenuation of different energy plutonium gamma rays to correct for sample attenuation. It also includes a good discussion of the operation of $\mathrm{Ge}(\mathrm{Li})$ detectors and their associated electronics. This report should be read in conjunction with the next report which describes some of the assumptions and limitations of the "contrast method" and reports some experimental results on plutonium waste standards.

5.3. T. D. Reilly and J. L. Parker, Los Alamos Scientific Laboratory A-1 Progress Roport LA-5197-PR (1972), pp. 15-18. The title of this article is "The Contrast Method-Gamma Attenuation Correction Based on Differential Absorption."

5.4. "Proceedings of the Symposium on Passive Gamma Ray Assay," W. A. Higinbotham, Ed. This conference was held in Germantown, MD, November 19-20, 1970. This includes short reports from 12 nuclear facilities on their use of gamma assay equipment. Though somewhat outdated, it gives useful information on the gamma assay techniques in use at United States nuclear facilities. 


\section{BNRICEMIXIT MIASURAMTNTS}

\subsection{Introduction}

The provious chaptor showe that the anay of $235 \mathrm{y}$ is very difficult becauwe of the high ealf-attonuation of uranium at $186 \mathrm{koV}$. The mas attonuation confticiont of uranium at $186 \mathrm{koV}$ is about $1.5 \mathrm{~cm}^{2} / \mathrm{g}$, which is nearly 12 times greater than the average for all eloment bolow atomic numbur $\mathrm{Z}=30$ (this is about $0.13 \mathrm{~cm}^{2} / \mathrm{g}$ ). This very circumatonce makes the mocurrement of uranium oxrichnent relatively cary for many practical situations. The fundamental relationahip is thin: The intennity of 186-keV gamma raye omitted from a fixed area of a thick cample of uranium is proportional to the enrichment of the samplo. "Thick" in this context means several mean free pathe at $186 \mathrm{koV}$. This is unally thin phycically; $2 \mathrm{~mm}$ of uronium motal is almost infinitoly thick to these gamma rays. Numerous syetems have mecenfully applied this concept with a varioty of electronice and detectors to a wide range of problems. Thase include enrichment moarurements of tuel pins, fuel plates, contrinere of $\mathrm{UO}_{2}$, cylinders of $U_{F_{6}}$, and in-line monitoring of liquid $U F_{6}$.

A detailed discuscion of gamma-ray enrichment measurements is omitted here because the rubject is well covered in the linted references. ${ }^{6.1-6.4}$ The Rull report ${ }^{6.1}$ should be read by anyone interented in enrichment measurements. This chaplor will be confined to a simple derivation of the fundamenta! rolationship and a description of how it may be applied to measuring uranium concentration and blending ratios as well as to enrichment measurements. A few comments will be made about dotermining plutonium isotopic distributions.

\subsection{The Fundamental Relation}

The detector illustrated in Fig. 6.1 viewe a fired area of a uranium sample through an appropriate collimator. The sample is infinitely thick for $186-k 0 V$ gamma rays (transmistion $\leq 0.01$ ) and consists of a uniform mixture of uranium and matrix (everything eleo). The density of uranium is $\rho_{\mathrm{u}}$, and matrix $\rho_{\mathrm{m}}$. The mass attenuation coefficients at this energy are $\mu_{u}$ and $\mu_{m}$. The count rate can be expresed by the integral relation

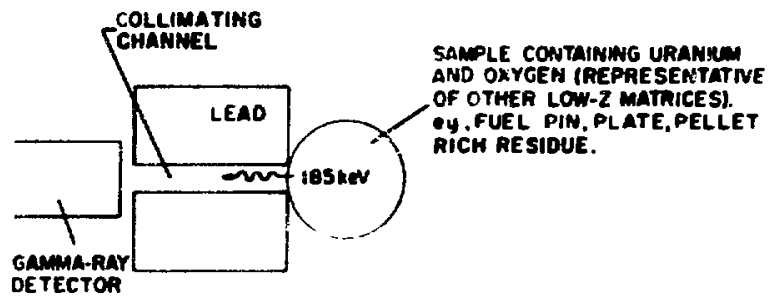

Fig. 6.1.

Silhematic illustration of uranium enrichmem mowuremem.

$C R=I \Gamma \in A \rho_{u} \int_{0}^{\infty} \exp \left[-\left(p_{u} \mu_{u}+p_{m^{\mu}} m^{\prime x}\right] d x,(6.1)\right.$

where $I={ }^{235} \mathrm{U}$ enrichment,

E - total efficiency of counting system,

5 specific activity of ${ }^{235} \mathrm{U}\left(4.3 \times 10^{4} / \mathrm{g}-\mathrm{s}\right)$,

A area of sample viewed, and

$x$ distance (within sample) to front edge of sample.

This relationehip assumew that the variation in sample-to-dotector distance from different part of the sample may be ignored ( $c=$ conotant, independent of $x$ ). It aleo ignores any attenuation by eample packaging. For many situations, this relationship gives an adequate description of the problem. The integral is savily evaluated and yielda the exprewion

$$
C R=\frac{K \cdot I}{1+\frac{\mu_{m}}{\mu_{u}} \frac{\rho_{m}}{\rho_{u}}} \text {. }
$$

where $R=\Gamma e A / \mu_{u}, \alpha$ constent which is determined by calibration with a known standard. 
A thorough understanding of Eq. (6.2) is esential to measure onrichmont. There are other considerations of equipment, procedure, wall corractions, unusual geometries, etc., which are not represented in Eq. (6.2). Many of these are ccrered in Ref. 6.1. However, Eq. (6.2) is the fundamental relation that contains all the basic information for measuring enrichment. Three areas of application exist as defined by differeni values of the ratios $\mu_{\mathrm{m}} / \mu_{\mathrm{u}}$ and $\rho_{\mathrm{m}} / \rho_{\mathrm{u}}$, which will be discussed separately.

\subsection{Enrichment Region}

The important parameter is the value of $\mu_{\mathrm{m}} \rho_{\mathrm{m}} / \mu_{\mathrm{u}} \rho_{\mathrm{u}}$. If $\mu_{\mathrm{m}} p_{\mathrm{m}} / \mu_{\mathrm{v}} \rho_{\mathrm{u}} \leq 0.1$, the observed count rate (CR) is proportional to the enrichment with an error of $10 \%$ or less. For matrix material with $\mathrm{Z} \leq 30$, $\mu_{\mathrm{m}} / \mu_{\mathrm{v}} \sim 0.08$. Thus, $\mu_{\mathrm{m}} \rho_{\mathrm{m}} / \mu_{\mathrm{w}} \rho_{\mathrm{u}} \leq 0.1$ if $\rho_{\mathrm{m}} / \rho_{\mathrm{u}} \leq 1$. A half-and-half mixture of uranium and low- $Z$ material would give less than $10 \%$ fewer counts than a slab of uranium metal having the same enrichment. A wide range of compounds and mixtures may be measured by comparison to a single standard with only small corrections for the variation in composition. If a standard and a series of unknown samples are known to be the same compound or mixture, the enrichments will be directly proportional to the observed rates. For example, if both standard and samples are pure $\mathrm{UF}_{6}$, the term $\left(1+\mu_{\mathrm{m}} \mu_{\mathrm{m}} / \mu_{\mathrm{u}} \rho_{\mathrm{u}}\right)$ can be neglected. If the standard were $\mathrm{UO}_{2}$ and the unknowns $\mathrm{UF}_{6}$, a small correction might be applied to the calibration to occount for the denominator of Eq. (6.2). If the uranium concentration is known, the denominator can be evaluated directly and the range of the enrichment measurement can be extended to lower concentrations. In favorable circumstances, accurate enrichment measurements he ye been made on residues with less than $10 \%$ uranium by weight

\subsection{Concentration Region}

Now consider the case where $\mu_{\mathrm{m}} \rho_{\mathrm{m}} / \mu_{\mathrm{u}} \rho_{\mathrm{u}} \geq 10$. With an error of $10 \%$ or less, Eq. (6.2) becomes

$$
\mathrm{CR} \approx \mathrm{K}^{\circ} \mathrm{I} \rho_{\mathrm{u}} / \rho_{\mathrm{m}}
$$

Thus, if the enrichment ( $I$ ) is known, the count rate is directly proportional to the relative concentration of uranium and matrix. Because $\mu_{\mathrm{m}} / \mu_{\mathrm{u}} \sim 0.08(\mathrm{Z} \leq 30)$, $\mu_{m} \rho_{m} / \mu_{u} \rho_{u} \geq 10$ if $\rho_{m} / \rho_{u} \gtrsim 100$. Consider a dilute uranium solution (lese than $1 \%$ uranium by weight). If the enrichment is known, the uranium concentration can be computed directly from the 186-keV count rate. The total uranium can then be computed from the net weight of the solution. For dilute mixtures, Eq. (6.3) might be said to define a "concentration meter." It is important to remember that Eq. (6.2) is based on the assumption that the sample is infinitely attenuating to the gamma rays of interest (186 $\mathrm{keV}$ ). The concentration limil occurs when the matrix is the dominant part of the attenuation. The mean tree path of 186-keV gamma rays in low-Z material can be quite long $\left(\sim 7 \mathrm{~cm}\right.$ in $\left.\mathrm{H}_{2} \mathrm{O}\right)$ so that more material is required to define a thick sample. Concentration measurements should be attempted with caution in cases where matrix density or sample size varies.

\subsection{Mass Fraction for High-Z Mixtuxes}

Sometimes the matrix material is another high-Z element, e.g., thorium or plutonium. For hoth of these cases, $\mu_{\mathrm{m}}$ differs from $\mu_{\mathrm{u}}$ by $5 \%$ or less. Thus, with $a$ small error, $\mu_{\mathrm{m}} / \mu_{\mathrm{u}} \approx 1$, and Eq. (6.2) becomes

$$
\mathrm{CR} \approx \frac{\mathrm{K} \cdot \mathrm{I}}{\mathrm{I}+\rho_{\mathrm{m}} / \rho_{\mathrm{u}}} \quad \mathrm{K} \cdot \mathrm{I} \cdot \frac{\rho_{\mathrm{u}}}{\rho_{\mathrm{u}}+f_{\mathrm{m}}} \cdot(6.4)
$$

If the enrichment is known, the count becomes a measure of the uranium mass fraction $f$. This can be rewritten as

$$
C R \approx K \cdot I \cdot f \text {, }
$$

where

$$
f=\frac{\rho_{u}}{\rho_{u}+\rho_{m}}
$$

to emphasize the fact that the measured count rate is proportional to the product of the uranium enrichment and the uranium mass fraction. If either quantity is known, the other can be measured. This relation applies equally well to the measurement of plutonium isotopic ratios and to plutonium blending ratios in mixed oxide fuel. Note, however, that most work with uraniunn-plutonium mixtures will require a 
high resolution detector. Reference 6.2 discusses this case in more detail and accounts for the fact that $\mu_{\mathrm{m}} / \mu_{\mathrm{u}}=1$ is not strictly true.

\subsection{Summary of Relations}

( $\approx$ implies an error less than $10 \%$ )

Fundamental Relation

$$
\mathrm{CR}=\frac{K \cdot I}{1+\frac{{ }_{\mathrm{m}} \rho_{\mathrm{m}}}{\mu_{\mathrm{u}} \rho_{\mathrm{u}}}}
$$

\section{Enxichment Region}

$$
\begin{aligned}
C R \approx K . I & \text { if } \frac{\mu^{\prime} \rho_{m}}{\mu_{u} \rho_{u}} \leq 0.1 \\
& \text { for } Z \leq 30 \frac{\mu_{m}}{\mu_{u}} \sim 0.1 .
\end{aligned}
$$

the condition is met

$$
\text { if } \frac{\rho_{\mathrm{m}}}{\rho_{\mathrm{u}}} \leq 1 \text {. }
$$

\section{Concentration Region}

$$
\begin{gathered}
C R \approx K^{\prime} \cdot I \cdot \rho_{u} / \rho_{m} \\
\text { if } \frac{\mu_{m} \rho_{m}}{\mu_{u} \rho_{u}}=10
\end{gathered}
$$

$$
\text { for } \mathrm{Z} \leq 30 \text {, this holds if } \frac{\rho_{\mathrm{m}}}{\rho_{\mathrm{u}}} \gtrsim 100 \text {. }
$$

Mass Fraction for Hïigh-Z Mixturen

$$
\mathrm{CR} \approx \mathrm{K} \cdot \mathrm{I} \cdot \frac{\rho_{\mathrm{u}}}{\rho_{\mathrm{u}}+\rho_{\mathrm{m}}}
$$

$$
\text { if } \frac{\left|\mu_{m}-\mu_{u}\right|}{\mu_{u}} \leq 0.1 \text {. }
$$

This condition is met for $88 \leq \mathrm{Z} \leq \mathbf{9 6}$.

\subsection{Example 1. Measurement of Highiy Enrich- ed UF 6 Cylinders}

This problem is to verify the stated enrichment volues of three UF 6 cylinders (TYpe 5A, 13-cm-diam by 90-crn-high). The available equipment includes a $5.1-\mathrm{cm}$-diam by $2.5 \mathrm{~cm}$-thick $\mathrm{NaI}$ detector with a lead shield and collimator; c portable electronics package including a high-voltage supply, amplifier, two single-channel analyzers, and digital zate multipliers (DRM) for automatic background subtraction and $a$ portable ultrasonic thickness gauge. This equipment is mounted on a small cart as shown in Fig. 6.2.

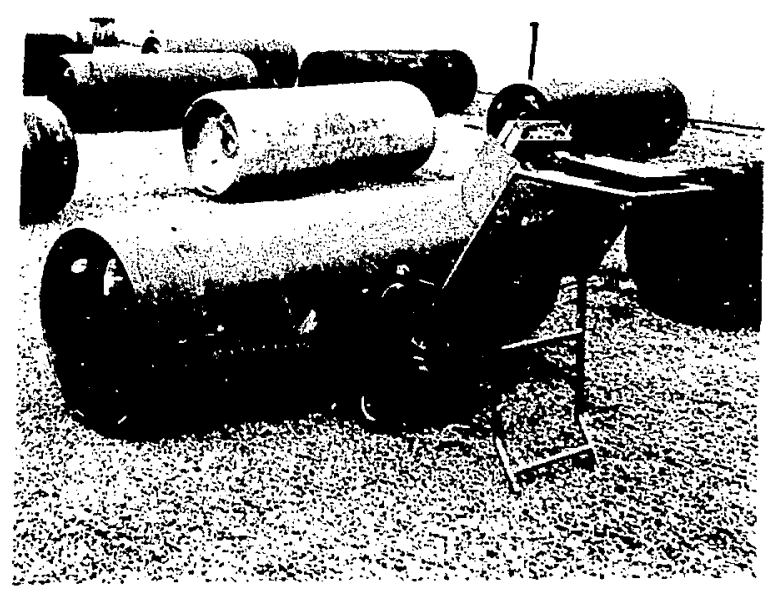

Fig. 6.2.

Gamma-ray enrichment measurement with Eberline SAM-II gamma unit and Branson ultrasonic thickness gauge. 
The verification procedure is to use one of the cylinders as a standard and measure the others relative to this. The detector is positioned $7.6 \mathrm{~cm}$ back from the front of the lead collimator which is held against the side of the cylinder. A $0.08-\mathrm{cm}$ cadmium foil is taped to the detector face to attenuate lowenergy $x$ rays. The two SCA's are set to count events in the 130 - to $210-\mathrm{keV}$ energy region (channel $A$ ) and the 230- to $310-\mathrm{keV}$ energy region (channel B). The net count rate is computed as

$$
R=A-1.5 B .
$$

The DRM's are set at 1.0 and 1.3 , so that $R$ is displayed directly. Several 6-s counts are made on each cylinder. The cylinder wall thickness at the gamma measurement point is measured with an ultrasonic thickness gauge. The wall thickness of $5 \mathrm{~A}$ cylinders is not uniform and errors of $5 \%$ or more can result if the variation is not taken into account. If a thickness gauge is not available, several gamma measurements should be made around the circumference of the cylinder and then averaged. The cylinder enrichment is computed from

$$
I-K R e^{1.17 x} \text {, }
$$

where

$$
\begin{aligned}
I \text { - cylinder enrichment }(\% & 235 \mathrm{~J}), \\
\mathrm{K}= & \text { calibration determined from standard, } \\
\mathrm{R}= & \text { observed net count }[\mathrm{Eq} .(6.5)] \text {, and } \\
\mathrm{X}= & \text { measured wali thickness (the effective } \\
& \text { absorption coefficient is } 1.17 \mathrm{~cm}^{-1} \text { for. } \\
& \text { Monel and } 0.95 \mathrm{~cm}^{-1} \text { for steel). }
\end{aligned}
$$

The measured results are presented in Table 6.1, which gives actual data and shows the accuracy that can be obtained for such measurements. If the wall thickness correction had been omitted, the deviations would be $+2.0 \%$ for Cylinder 2 and $-3.2 \%$ for Cylinder 3. In some cases, this correction will be larger. Recent measurements using the procedure and equipment described in this example show that highly enriched $\mathrm{UF}_{6}$ cylinders (type 5A) can be measured with a relative accuracy of better than $2 \%{ }^{6.5}$ The calibration procedure described in the next example can also be used for this problem. The measurement of larger, thick-walled, lowenrichment cylinders would be by the same procedure but would be subject to larger error due to the increasea absorbtion and poorer signal to background.

\subsection{Example 2: Enrichment Calibration Procedure}

This problem is to determine an independent calibration for ${ }^{235} \mathrm{U}$ enrichment measurements. The equipment is the same as for Example 1. Two uranium oxide standards of known enrichment ( 10.0 and $0.72 \%{ }^{235} \mathrm{U}$ ) are available for calibration. The material is contained in small cans, $10-\mathrm{cm}$-diam by $13-\mathrm{cm}$-high. The detector is positioned $7.6 \mathrm{~cm}$ from the front of the collimator, which is now supported in the vertical position. The can is positioned on top of the collimator so that the detecior does not view the edge of the can. The SCA windows are sel as before $(A=130-210 \mathrm{keV}, B=230-310 \mathrm{keV})$. Several 60-s counts are taken on each can. The average count is presented in Table 6.2.

The enrichment is assumed to obey the equation

$$
I=a A-b B .
$$

where $a, b$ ase constants determined by solving the following simultaneous equations.

$$
\begin{aligned}
& l_{1} \cdot \mathrm{a} A\left(1_{1}\right)-\mathrm{b} I B\left(I_{1}\right) \\
& l_{2} \cdot \text { a } A\left(1_{2}\right)-b 13\left(1_{2}\right),
\end{aligned}
$$

and $I_{1}, I_{2}$ are the known enrichments $10.0,0.72$ $\%{ }^{235} \mathrm{U}$. The solution of this is given as

$$
\begin{aligned}
& \text { a : } \frac{I_{1} \cdot B\left(I_{2}\right)-1_{2} \cdot B\left(I_{1}\right)}{D} \\
& b=\frac{I_{1} \cdot A\left(I_{2}\right)-I_{2} \cdot A\left(I_{1}\right)}{D} \\
& D=A\left(I_{1}\right) \cdot B\left(I_{2}\right)-A\left(I_{2}\right) \cdot B\left(I_{1}\right) .
\end{aligned}
$$


TABLE 6.1

\section{MEASUREMEAT OF UF CYLINDERS}

\begin{tabular}{|c|c|c|c|c|c|}
\hline Cyl & $I_{\text {tar }}(\%)$ & $\boldsymbol{R}(60)$ & $X(\mathrm{~cm})$ & $I_{m}(\%)$ & {$\left[\left(I_{m}-I_{\text {taq }}\right)\right] \times 100 \%$} \\
\hline $\begin{array}{l}1^{a} \\
2 \\
3\end{array}$ & $\begin{array}{l}97.6 \\
46.8 \\
19.9\end{array}$ & $\begin{array}{c}29030 \\
14210 \\
5732\end{array}$ & $\begin{array}{l}0.645 \\
0.622 \\
0.660\end{array}$ & $\begin{array}{l}97.6 \\
46.5 \\
19.6\end{array}$ & $\begin{array}{c}0 \\
-0.6 \\
-1.5\end{array}$ \\
\hline
\end{tabular}

Substituting the values from Table 6.2,

$$
\begin{aligned}
& D=(80125)(10902)-(18146)(9790)=6.96 \times 10^{8} \\
& a=\frac{(10)(10902)-(0.72)(9790)}{6.96 \times 10^{8}}=1.47 \times 10^{-4} \\
& b=\frac{(10)(18146)-(0.72)(80125)}{6.96 \times 10^{8}}-1.78 \times 10^{-4} .
\end{aligned}
$$

With the constants determined, other cans of uranium oxide can now be measured directly. An unknown can is measured and counts $A=45090$, $B$ $=10100$. The measured enrichment of this can is given by Eq. (6.8).

$$
\begin{aligned}
J & =\left(1.47 \times 10^{-4}\right)(45090)-\left(1.78 \times 10^{-4}\right)(10100) \\
& =4.8 \% .
\end{aligned}
$$

\begin{tabular}{|c|c|c|}
\hline $\begin{array}{c}\text { Enxichment } \\
\left(\% \%^{235} U\right)\end{array}$ & $A$ (counts) & B(counts) \\
\hline $\begin{array}{c}10.0 \\
0.72\end{array}$ & $\begin{array}{l}80125 \\
18146\end{array}$ & $\begin{array}{r}9790 \\
10902\end{array}$ \\
\hline
\end{tabular}

TABLE 6.2

\section{MEASUREMENT OF URANIUM OXIDE ENRICHMENT STANDARDS}

For the specified equipment, the constants can be set on the DRM and the solution of Eq. (6.8) will be displayed directly in $\%{ }^{235} \mathrm{U}$.

This procedure can be adapted to calibrate the instrument for $\mathrm{UF}_{6}$ cylinder measurements. The collimator restricts the deteitor view to a fixed area whether the sample is a small can or a large cylinder. It is advisable to perform the calibration with an iron or nickel plate of the approximate cylinder wall thickness under the can. This more closely approximates the cylinder measurement so that relatively small corrections are required for variations in wall thickness.

\section{REFERENCES}

6.1. L. A. Kull and A. O. Ginaven, "Guidelines for Gamma-Ray Spectroscopy Measurements of ${ }^{235} \mathrm{U}$ Enrichment," Brookhaven National Laboratory report BNL-56414 (1974). This was prepared for the USAEC Directorate of Regulatory Standards and was used as the hasis for Regulatory Guide 5.21. It is a rather complete treatment of the subject of uranium enrichment measurements.

6.2. T. D. Reilly, R. B. Walton, and J. L. Parker, Los Alamos Scientific Laboratory A-1 Progress Report LA-4605-MS (1970), pp. 19-21. The title of this article is "The 'Enrichment Meter'-A Simple Method for Measuring Isotopic Enrichment." This is the original reference to the "enrichment meter" principle and the fundamental relation [Eq. (6.2)]. It includes a discussion of the measurement of $\mathrm{Pu}-\mathrm{U}$ blending ratios. Other related progress report references on concentration measurements are Refs. 6.3 and 6.4. 
6.3. M. M. Thorpe, R. B. Walton, and L. V. East, Los Alamos Scientific Laboratory A-1 Progress Report LA-4794-MS (1971), pp. 14-16. The title of this article is "Assay of Uranium Solution Storage Tanks."

6.4. J. L. Purker and T. D. Reilly, Los Alanios Scientific Laboratory A.I Progress Peport LA-5197-PR (1972), Fp. 11-12. The title of this article is "The Enrichment Meter as a Concentration Meter."
6.5. R. B. Walton, T. D. Reilly, J. L. Parker, J. H. Menzel, E. D. Marshall, and L. W. Fields, "Measurements of $\mathrm{UH}_{6}$ Cylinders with Portable Instrumente," Nucl. Technol. 21, 133-148 (1974). This reparts measurements of high and low enriched $\mathrm{UF}_{6}$ product cylindere using gamma enrichment measurements ( $\mathrm{Nal}+$ ultrasonic measurement of cylinder wall thickness) and several neutron measurement techniques. 


\section{NEUTRON PRODUCTION AND APPLICABLE SIGNATURES}

\subsection{Introduction}

There are some measurement problems for which the counting of passive neutrons is an cttractive method of assay. These situations uaually involve one or more of the following circumstances.

1. Well-characterized material, both isotopic and chemical composition, e.g., pure $\mathrm{PuO}_{2}$ or enriched $\mathrm{UF}_{6}$

2. Large quantities of relatively dense material, e.g., plutonium buttons.

3. Material with high gamma backgrounds, e.g., slightly irradiated plutonium fuel.

Care must be exercised in using total neutron counting as an assay tool; but for appropriate cases, it provides a simple assay method.

Neutrons from fissionable material, other than those produced by induced fission, are emitted as a result of two processes: spontaneous fission and the interaction of alpha radiation with light elements. Under proper circumstances, neutrons from either process can be a useful assay signature.

\subsection{Alpha-Induced Neutron Emission}

Historically, the first free neutrons observed by man were produced by the interaction of alpha particles from the decay of polonium on beryllium. The nuclear reaction involved is

$$
{ }^{9} \mathrm{Be}+{ }^{4} \mathrm{He} \rightarrow{ }^{12} \mathrm{C}+\mathrm{n}+5.708 \mathrm{MeV} .
$$

The energy of the alpha particle (5.3 $\mathrm{MeV}$ for ${ }^{210} \mathrm{Po}$ ) is added to the indicated energy released in the reaction. Most of this total energy is released as the kinetic energy of the neutron, resulting in neutrons with energies up to about $10 \mathrm{MeV}$. Neutrons of lesser energy are produced when the residual ${ }^{12} \mathrm{C}$ nucleus is left in an excited state of 4.43 or $7.65 \mathrm{MeV}$, which subsequently decays by gamma radiation. The emitted neutron energy is also reduced if the alpha particle is slowed down by atomic collisions before entering into the reaction with the beryllium nucleus. The result of all these processes is a fairly continuous spectrum of neutron with a maximum intensity around $4.5 \mathrm{MeV}$, tailing off to zero intensity at mazimum energy and at vory low energies. ${ }^{7.1}$ The maximum alpha energy of various fiasioncible nuclides is indicated in Table 7.2, column 4. Depen. ding on the light element combined with the fissionable nuclide, the maximum neutron ezergy can vary from approximately I $\mathrm{MeV}(\mathrm{Li})$ up to 10 $\mathrm{MeV}(\mathrm{Be})^{7.2}$

Thus, the neutron eignature does not consist of narrow, well-defined lines as do the gamma signaturez. As a consequence, it is not possible to measure uniquely the amount of a given isotope in a sample without additional information.

Many light elements have isotopes that yield neutrons from the $(\alpha, n)$ reaction when bombarded by energetic alpho particles. A partiai list of such elements, and the yield of neutrons emitted per $10^{6}$ alpha particles, is given in Table 7.1, assuming polonium alpha particles strike $\alpha$ thick target of the element. The actual yield of neutrons is very dependent upon the energy of the alpha particles. ${ }^{7.3}$ Table 7.2 lists known heavy isotopes of interest to the nuclear fuel cycle, their decay rates and alpha energies, and expected neutron yields from the ${ }^{9} \mathrm{Be}(\alpha, n)^{12} \mathrm{C}$ reaction. Note that because tissionable fuel materials are often found in the manufacturing cycle as pure oxides or fluorides, the reactions with these two light elements are particularly interesting from an assay standpoint. Table 7.3 lists the data relevant to these compounds.

The maximum yield of neutrons to be expected from any combination of tissionable material and light elements can be obtained by taking the neutron yield for alphas on berylium from the last column of Table 7.2 and multiplying it by the third (yield relative to beryllium) column of Table 7.1. In all cases the yield actually obtained will be less because of the slowing down effect of alpha particles between the time they leave their parent nuclei and the time that they react with light-element nuclei. The amount of this slowing down depends upon the ratio of the alpha-emitting isotope to the particular light element, the presence of nonproductive or lesser productive heavy isotopes, and the physical mixing of material in the sample.

The counting of $(\alpha, n)$ neutrons as a quantitative assay signature is restricted to those cases where the material to be assayed is homogeneous and of known 
TABLE 7.1

$(x, n)$ NEUTRON YIELDS FOR

VARIOUS LIGHT ELEMENTS

\begin{tabular}{|c|c|c|}
\hline Element X & $\begin{array}{c}\text { Noutron Yiold } \\
\text { per } 10^{8} \alpha^{\prime} s^{\alpha} \\
\end{array}$ & $\begin{array}{c}\text { Yield Rolative to } \\
\text { Boryllium }\end{array}$ \\
\hline $\begin{array}{l}\mathrm{Be} \\
\mathrm{B} \\
\mathrm{F} \\
\mathrm{Li} \\
\mathrm{Na} \\
\mathrm{Mg} \\
\mathrm{Al} \\
\mathrm{As} \\
\mathrm{Si} \\
\mathrm{Cl} \\
\mathrm{C} \\
\mathrm{O} \\
\mathrm{N}\end{array}$ & $\begin{array}{l}80 \\
24 \\
12 \\
2.6 \\
1.5 \\
1.4 \\
0.74 \\
0.38 \\
0.16 \\
0.11 \\
0.11 \\
0.07 \\
0.01\end{array}$ & $\begin{array}{l}1.00 \\
3.0 \times 10^{-1} \\
1.5 \times 10^{-1} \\
3.6 \times 10^{-2} \\
1.9 \times 10^{-2} \\
1.8 \times 10^{-2} \\
9.3 \times 10^{-3} \\
4.8 \times 10^{-3} \\
2.0 \times 10^{-3} \\
1.4 \times 10^{-3} \\
1.4 \times 10^{-3} \\
8.8 \times 10^{-4} \\
1.25 \times 10^{-4}\end{array}$ \\
\hline
\end{tabular}

व. H. Roberts, "Neutron Yields of Several Light Elements Bombarded with Polonium Alpha Particles," Los Alamos Scientific Laboratory internal documen: (1944).

chemical composition. Becausa numerous isotopic combinations could be made up to give the same neutron output, counting $(\alpha, n)$ neutrons must be considered as a verification or comparison check only and must not be relied upon for the quantitative assay of parcels with totally unknown contents.

\subsection{Neutzons from Spontaneous Fission}

Spontaneous fission is a form of breakdown of unstable heavy nuclides which competes with alpha, beta, and gamma radiation as a means for these nuclides to decay into more stable species. All fissionable isotopes undergo spontaneous fission to some degree, although in only of few cases is the rate of fission great enough to yield a quantity of neutrons adequate for assay purposes.

As is the case for the more familiar neutroninduced fission, when a nucleus undergoes spontoneous fission, it breaks up into two smaller nuclei plus a number of free neutrons. The breakup is a statistical process in which the two fission fragments can have a broad range of nuclear masses; therefore, the number of leftover free neutrons released is indeterminate for a single fission event.
Averaged over many fissions of a single isotope, however, the number of neutrons $\bar{v}$ released by spontaneous fission of that isotope is well determined by the nature of the fission process. Because a spontaneously fissioning nucleus has one less neutron than a nucleus of the same isotope which is undergoing fission after absorption of a neutron, $\bar{v}$ for spontaneous fission of a given isotope is always somewhat lower than $\bar{v}$ for neutron-induced fission. Figure 7.1 shows a typical energy spectrum of fission neutrons, where the neutrons have $a$ broad range of energies peaking below $1 \mathrm{MeV}$ with an average energy between 1 and $2 \mathrm{MeV}$.

Table 7.4 lists spontaneous fission half-lives, prompt-neutron yields per fission, and spontaneous fission rates per gram of fissionable isotopes found in the fuel cycle. Note that the fertile species ${ }^{238} U$ and ${ }^{240} \mathrm{Pu}$ have higher spontaneous fission rates than the fissile isotopes ${ }^{235} \mathrm{U}$ and ${ }^{239} \mathrm{Pu}$.

$$
\frac{\left.{ }_{S F}{ }^{238} U\right)}{\left.\lambda_{S F} r^{235} U\right)} 18
$$

and 


\section{ATPLA IND $(a, n)$ NEUTRON EMISSION}

FROM FISSIONABLE ISOTOPES

\begin{tabular}{|c|c|c|c|c|c|}
\hline Isotope & $\underset{\text { (yr) }}{\alpha \text { Hollf-Lito }}$ & $\alpha / s / g$ & $\begin{array}{l}\text { Approximate } \\
\text { a Energy }\end{array}$ & $\begin{array}{l}\text { Noutzons/a/ } \\
10^{6} \alpha^{\prime}=\text { on } B_{0}^{b}\end{array}$ & $\begin{array}{c}\text { Neutrons/s/g } \\
\text { on } \mathrm{Bo}^{\mathrm{C}}\end{array}$ \\
\hline $\begin{array}{l}{ }^{233} \mathrm{U} \\
234 \mathrm{U} \\
235 \mathrm{U} \\
236 \mathrm{U} \\
238 \mathrm{U} \\
238_{\mathrm{Pu}} \\
239_{\mathrm{Pu}} \\
240 \mathrm{Pu} \\
211_{\mathrm{Pu}} \\
241_{\mathrm{Am}} \\
242_{\mathrm{Pu}}\end{array}$ & $\begin{array}{c}1.62 \times 10^{5} \\
2.47 \times 10^{5} \\
7.1 \times 10^{8} \\
2.4 \times 10^{7} \\
4.5 \times 10^{9} \\
88 \\
2.4 \times 10^{4} \\
6.6 \times 10^{3} \\
14.4^{d} \\
432 \\
3.79 \times 10^{5}\end{array}$ & $\begin{array}{l}3.5 \times 10^{8} \\
2.3 \times 10^{8} \\
7.9 \times 10^{4} \\
2.3 \times 10^{6} \\
12 \times 10^{4} \\
6.4 \times 10^{11} \\
2.3 \times 10^{9} \\
8.4 \times 10^{9} \\
9.4 \times 10^{7} \\
1.3 \times 10^{11} \\
1.4 \times 10^{8}\end{array}$ & $\begin{array}{l}4.8 \\
4.7 \\
4.3 \text { to } 4.4 \\
4.5 \\
4.2 \\
5.5 \\
5.1 \text { to } 5.2 \\
5.1 \text { to } 5.2 \\
4.9 \\
5.5 \\
4.9\end{array}$ & $\begin{array}{l}48 \\
45 \\
30 \\
35 \\
28 \\
72 \\
60 \\
60 \\
50 \\
72 \\
50\end{array}$ & $\begin{array}{l}1.7 \times 10^{4} \\
1.04 \times 10^{4} \\
2.4 \\
81 \\
0.3 \\
4.5 \times 10^{7} \\
1.38 \times 10^{5} \\
5.0 \times 10^{5} \\
4700 \\
9.4 \times 10^{E} \\
7000\end{array}$ \\
\hline
\end{tabular}

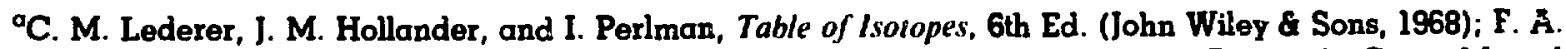
$\mathrm{O}^{\prime}$ Hara et al., "Determination of $\mathrm{Pu}$ in Reactor Feed Materials," Monsanto Research Corp, Mound Laboratory report MLM-1798 (1972).

${ }^{b}$ Calculated from A. O. Hanson, Chapter 1A, in Fast Neutron Physics, J. B. Marion and J. L. Fowler, Eds. (Interscience, 1960), Vol. 1, p. 11, Fig. 7.

' $[$ Column (5) x Column (3) $] \div 10^{6}$.

do.00246\% a branching ratio: most of the time it bela decays to ${ }^{241} \mathrm{Am}$.

TABLE 7.3

\section{$(\alpha, n)$ YIELDS FROM OXIDES AND FLUORIDES}

\begin{tabular}{|c|c|}
\hline Material & $\begin{array}{c}\text { Yield } \\
\text { (neutrons } / \mathrm{g} / \mathrm{g} \text { ) }\end{array}$ \\
\hline${ }^{234} \mathrm{UO}_{2}$ & $\sim 14$ \\
\hline${ }_{234}^{234} \mathrm{UF}_{6}$ & $5.8 \times 10^{2}$ \\
\hline${ }^{238} \mathrm{UF}_{6}$ & $\begin{array}{l}12.2 \times 10^{-2} \\
12.9 \times 10^{-3}\end{array}$ \\
\hline${ }^{238} \mathrm{PuO}_{2}$ & $1.4 \times 10^{4}$ \\
\hline $\begin{array}{l}{ }^{238} \mathrm{PuF}_{4} \\
{ }^{239} \mathrm{PuO}_{2}\end{array}$ & $\begin{array}{c}2.1 \times 10^{6} \\
45\end{array}$ \\
\hline${ }^{239} \mathrm{PuF}_{4}$ & 4300 \\
\hline${ }^{240} \mathrm{PuO}_{2}$ & 170 \\
\hline${ }^{240} \mathrm{PuF}_{4}$ & $\begin{array}{c}1.6 \times 10^{4} \\
\sim 10\end{array}$ \\
\hline${ }^{241} \mathrm{AmO}_{2}$ & 3754 \\
\hline${ }^{242} \mathrm{PuO}_{2}$ & $\sim 10$ \\
\hline
\end{tabular}

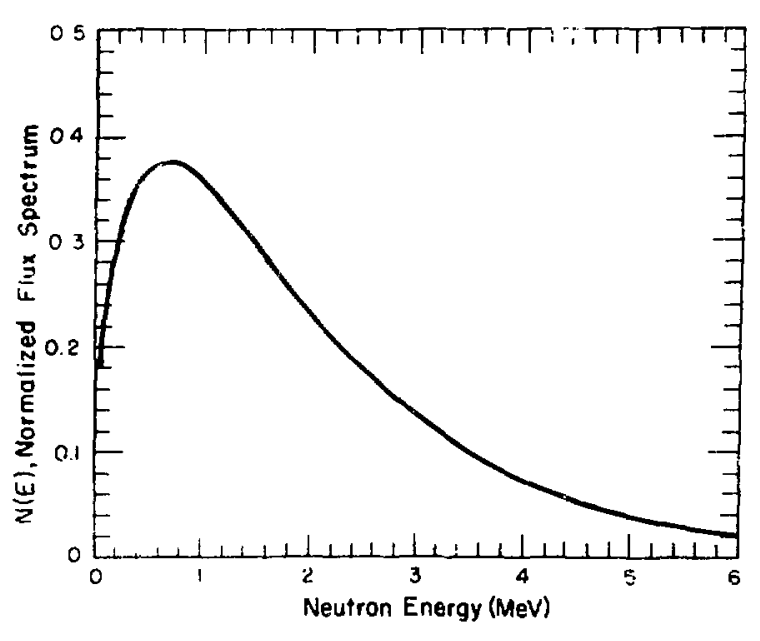

Fig. 7.1.

Tipical energ! spe:trum of fission neutrons. 


$$
\frac{{ }^{\lambda} \mathrm{SF}^{\left({ }^{240} \mathrm{Pu}\right)}}{{ }_{\left.{ }_{\mathrm{SF}}{ }^{239} \mathrm{Pu}\right)}}=4.7 \times 10^{4} .
$$

where $\lambda$ is the disintegration constant, that is, number of fissions per unit time.

Spontaneous fision is a proces that occurs entireIy within one nucleus. The $(\alpha, n)$ reaction, however, involves traneport of the alpha particles through the material being invertigated. Whereas the rate of emission of $(a, n)$ neutrons is highly dependent upon the nature of the matrix containing the fircionable material boing asscyed, the rate of neutron emission from the spontaneous fission process depends only upon the nuclear properties and the quantities of the fissioning isptopes present. Although the number of neutrons detected from a sample will be influenced by neutron absorption and scattering within the sample, these effects are in general far less severe than the alpha-attenuation effects that influence the yield from $(\alpha, n)$ reactions. Therefore, for accurate assay by passive neutron counting, one usually selects the spontaneous-fission neutrons, discriminating against $(\alpha, n)$ neutrons by requiring that detected neutrons be in coincident pairs. Even then it is necessary to know the isotopic composition to arrive at a measure of the tissile components.

\subsection{Interaction of Neutrons with Matter}

The interaction probability of neutrons passing through a slab of material is expressed by an equation identical in form to Eq. (1.7), namely,

$$
N=N_{0} e^{-\mu \rho t}
$$

However, the practice is to express $\mu_{m}$ in the following form.

$$
\mu_{\mathrm{m}} \frac{A_{o}}{A} \sigma\left(E_{n} / \mathrm{cm}^{2} / \mathrm{g},\right.
$$

where

$$
A_{0} \equiv \text { Avogadro's number }=0.6023 \times 10^{24} .
$$

$$
A \equiv \text { atomic weight, and }
$$

\begin{tabular}{|c|c|c|c|}
\hline Isotope & $\begin{array}{l}\text { SF Falf- } \\
\text { Life }\left(\mathrm{y}^{\mathrm{x}}\right)^{\mathrm{a}}\end{array}$ & $\begin{array}{c}\bar{v} \\
(\mathrm{SF}) \\
\end{array}$ & $S F / g-s$ \\
\hline $\begin{array}{l}{ }^{232} \mathrm{Th} \\
{ }^{234} \mathrm{U} \\
{ }^{235} \mathrm{U} \\
{ }^{236} \mathrm{U} \\
{ }^{238} \mathrm{U} \\
{ }^{238} \mathrm{Pu} \\
{ }^{239} \mathrm{Pu} \\
240 \mathrm{Pu} \\
241 \mathrm{Pu} \\
{ }^{242} \mathrm{Pu} \\
{ }^{241} \mathrm{Am} \\
{ }^{252} \mathrm{Cf}\end{array}$ & $\begin{array}{l}1.4 \times 10^{18} \\
2.0 \times 10^{16} \\
1.9 \times 10^{17} \\
2 \times 10^{16} \\
9.86 \times 10^{15^{b}} \\
4.9 \times 10^{10} \\
5.5 \times 10^{15} \\
1.17 \times 10^{11^{\mathrm{c}}} \\
5.0 \times 10^{15^{\mathrm{e}}} \\
6.8 \times 10^{10^{\mathrm{e}}} \\
2 \times 10^{14^{\mathrm{f}}} \\
86^{\mathrm{h}}\end{array}$ & $\begin{array}{l}\sim 2 \\
\sim 2 \\
\sim 2 \\
1.95^{d} \\
2.26^{d} \\
2.2^{1} \\
2.17^{c} \\
2.2 \\
2.16 \\
2.3^{9} \\
3.8\end{array}$ & $\begin{array}{l}4.1 \times 10^{-5} \\
2.8 \times 10^{-3} \\
2.56 \times 10^{-4} \\
2.8 \times 10^{-3} \\
5.64 \times 10^{-3} \\
1.1 \times 10^{3} \\
1.0 \times 10^{-2} \\
4.71 \times 10^{2} \\
1.1 \times 10^{-2} \\
8.0 \times 10^{2} \\
0.27 \\
6.14 \times 10^{11}\end{array}$ \\
\hline
\end{tabular}

$\sigma \equiv$ cross section with units of $\mathrm{cm}^{2} /$ atom.
TABLE 7.4

\section{SPOONTANEOUS FISSION OF FISSIONABLE ISOTOPES}

${ }^{a}$ C. M. Lederer, I. M. Hollander, and I. Perlman, Table of Isotopes, 6th Ed., (John Wiley \& Sons, 1968); see also A. Ghiorso, "Spontaneous Fission Correlations," Proceedings of the International Conference on the Peaceful Uses of Atomic Energy-Geneva, 1955, 7, p. 15.

bJ. H. Roberts, R. Gold, and R. J. Armani, "Spontaneous Fission Decay Constant of 238प," Phys. Rev. 174, 1482 (1968).

'P. Fieldhouse, D. S. Mather, and E. R. Cilliford, "The Spontaneous Fission Half-Life of ${ }^{240} \mathrm{Pu}^{\text {," J. }}$ Nucl. Energy 21, 749 (1967).

dC. J. Orth, "The Average Number of Neutrons Emitted in the Spontaneous Fission of Some EvenEven Heavy Nuclides," Nucl. Sci. Eng. 43, 54 (1971) and listed references.

'M. Taherzadeh, "Neutron Radiation Characteristics of $\mathrm{PuO}_{2}$ Fuel," JPL Technical Report 32-1555 (1972).

'Private communication, R. C. Smith of Batelle Northwest to Eugene Weinstock of TSO, March 16, 1970.

"Quoted as "estimated" in reference $f$.

hA. Prince, "Nuclear and Physical Properties of ${ }^{252} \mathrm{Cf}$," Brookhaven National Laboratory report BNL 50168 (T-530) (1969). 
The concept of cross section refers to the effective target area a nucleus presents to the approaching neutron. Just as in archery, the larger the target the higher the probability of intercction. Crosesections are commonly given in units of barns, where $1 \mathrm{~b}$ equals $10^{-24} \mathrm{~cm}^{2}$. Equation (7.1) then simplities to

$$
\mu_{m}=\frac{0.6023}{A} \sigma\left(E_{n}\right)
$$

where $\sigma$ is given in barns. The quantity $\mu_{m} \rho$ is given the name macroscopic cross section with the symbol ¿. At thermal energy the absorption cross section for plutonium is $\sim 1000 \mathrm{~b}$. Therefore the transmission through $0.0254 \mathrm{~cm}(0.010$ in.) of piutonium metal ( $\rho$ $=18 \mathrm{~g} / \mathrm{cm}^{3}$ ) is

$$
\begin{gathered}
\mu_{\mathrm{m}} \rho l=\frac{(0.6)(1000)(18)(0.01)(2.54)}{239}=1.15 \\
\frac{\mathrm{N}}{\mathrm{N}_{\mathrm{o}}}=\mathrm{e}^{-1.15}=0.32=\begin{array}{l}
\text { number of neutrons that pass } \\
\text { through the foil without inter- } \\
\text { acting. }
\end{array}
\end{gathered}
$$

Only $30 \%$ of incident thermal neutrons would be transmitted through a 10-mil foil. Cross-section values in general become smaller at higher neutron energies. For exampie, the absorption cross section for plutonium at $1 \mathrm{MeV}$ is $\sim 500$ times less than ther. mal value. This smaller cross section means greater penetrability through material. The total cross section is made up of partial cross sections corresponding to different reactions the neutron san have with the nucleus. (Neutrons interact with the nucleus and gamma rays inieract with atomic electrons.) Values for crose sections are tabulated in a series of books, BNL 325, 7.4 known as the "barn books." Table 7.5 presents a comparison of attenuations for $400-\mathrm{keV}$ gammas and 1-MeV neutrons, representative of signatures used in plutonium assay. The values in Table 7.5 show that neutron penetration at this energy is much higher than that of gamma rays through high-Z materials but is comparable for lower-Z materials. The values in Table 7.5 relate to the probability of any interaction occurring. Most of the neutron interactions do, in fact, still result in a neutron being detected. It is a situation analogous to the small-angle Compton scattering discussed in Sec. 1.2.f, only to a greater degree. This point will be discussed further at the end of Sec. 7.5.c.

\section{TABLE 7.5}

\begin{tabular}{|c|c|c|c|}
\hline Material & $\rho\left(\mathrm{g} / \mathrm{cm}^{3}\right)$ & $\begin{array}{l}\frac{1}{\mu_{Q}}(\mathrm{~cm}) \\
400-k e V \\
\text { Gammas }\end{array}$ & $\begin{array}{c}\frac{1}{\sum_{\text {total }}}(\mathrm{cm}) \\
\text { l-MeV } \\
\text { Neutrons }\end{array}$ \\
\hline $\begin{array}{l}\mathrm{H}_{2} \mathrm{O} \\
\mathrm{C} \\
\mathrm{Al} \\
\mathrm{Fe} \\
\mathrm{Pb} \\
\mathrm{U}\end{array}$ & $\begin{array}{l}1 \\
1.5 \\
2.70 \\
7.86 \\
11.35 \\
18\end{array}$ & $\begin{array}{l}9.9 \\
6.9 \\
4.1 \\
1.4 \\
0.43 \\
0.20\end{array}$ & $\begin{array}{l}2.0 \\
5.1 \\
5.6 \\
4.8 \\
6.1 \\
3.0\end{array}$ \\
\hline
\end{tabular}

\section{MEAN FFIEE PATH FOR 1-MeV NEUTRONS AND 400-keV GAMMA RAYS THROUGH VARIOUS MATERIALS}

\subsubsection{Interaction Processes}

It is possible to classify the kinds of neutron-matter interactions as follows.

1. Interactions in which the neutron is absorbed: $(n, \alpha)$ and $(n, \gamma)$ reactions.

2. Scattering processes resulting in neutron energy loss: elastic and inelastic scattering.

3. Frocesses leading to additional neutrons being emitted: $(n, 2 n)$ and fisrion processes.

$A$ rough analogy exists between neutron interactions and photoelectric, Compton scottering, and pair production processes. However, the relative importance of each process and its $Z$ and energy dependence will be significantly different for neutrons as compared to gammas. These differences make neutron counting preferable to gamma counting in some situations, and vice versa. ${ }^{7.5}$

7.5.b. Interactions in Which the Neutron Is Absorbed

The incoming neutron cun be absorbed by the nucleus and subsequently emit another particle or gamma ray. One example would be the $(n, a)$ reaction in which an clpha particle is emitted after the nentren is absorbed. This is the inverse of the $(\alpha, n)$ process discussed earlier. For most nuclides, this reaction is important only for neutron energies greater than approximately $5 \mathrm{MeV}$.

A second type of reacton is called "neutron capture" in which the nucleus absorbs a neutron followed by gamma-ray emirsion. This reaction is large of thermal or near-thermal neutron energies for some 
nuclides. Neutron capture is the mechaniom which makes breeding of fissionable material possible. For example,

$$
{ }^{238} \mathrm{U}+\mathrm{n} \rightarrow{ }^{239} \mathrm{U} \rightarrow{ }^{\mathrm{B}^{-}} \mathrm{239} \mathrm{Np} \stackrel{\mathrm{B}^{-}}{\rightarrow}{ }^{239} \mathrm{Pu} .
$$

Some materials, well known for their large capture cross sections, are used in reactor control rods or in shielding to remove neutrons. These materials, commonly called neutron poisons, include boron, cadmium, and gadolinium. The presence of poisons in combination with moderating material will significantly affect noutron asery results.

There are two absorption reactions of particular importance to neutron detection. These are

$$
\text { and } \begin{gathered}
{ }^{3} \mathrm{He}(n, p){ }^{3} \mathrm{H} \\
{ }^{10}{ }_{\mathrm{B}(n, a)}{ }^{7} \mathrm{Li}
\end{gathered}
$$

Further discussion of these reactions is included in Sec. 8.2, Gas Proportional Counter Tubes.

\section{5.c. Scattering Inteructions}

Neutron scattering by nuclei is a major interaction process for the energy range spanned by passive neutrone. (200 keV to several MeV). There are two kinds of posvible scattering interactions that can occur: elastic or inelastic. The relative number of each type of scattering interaction occurring at a particular energy is given by the respective cross-section values at that energy.

Filastic scattering can be thought of as a collision beiween billiard balls of different weights. For a heavier nucleus, such as uranium, the neutron can scatter with a large change in direction but with very little energy loss. As the scattering nucleus becomes lighter, the maximum energy the neutron can lose becomes larger. In a collinion with hydrogen, the neutron can lose up to all its energy in a single event. The amount of eneryy loss deperids on the angle of scattering as it did for Compton scattering. The expression for the merimum energy low, anclogous to the Compton edge, is

$$
\Delta E_{\max }=E_{n} \frac{4 m M}{(M+m)^{2}}
$$

where

$$
\begin{aligned}
\Delta E_{\max }= & \text { maximum energy loss of the } \\
\text { neutron } &
\end{aligned}
$$

$E_{n}=$ initial neutron energy .

$m=$ neutron mass, and

$M=$ mass of scattering nucleus .

Table 7.6 shows the percent maximum energy loss for. seven target nuclei.

Inelastic scatiering involves internal excitation of the target nucleus by the incident neutron. This process is something like throwing a stone agcinst a heavy bell. The bell does not recoil significantly, but vibrates to produce a ringing sound. The vibration is an internal excitation. Inelastic scattering is a mechanism whereby a neutron may lose a larger amount of energy in a collision with a heavy nucleus than would be possible through an elastic collision. For example, a l-MeV neutron will lose a maximum of $17 \mathrm{keV}$ in an elastic collision with ${ }^{238} \mathrm{U}$. A 1 $\mathrm{MeV}$ neutron will lose $300 \mathrm{keV}$ in every inelastic scattering with ${ }^{238} \mathrm{U}$. In general, inelastic scattering cross sections are largest for hocvier elements and for $E_{\mathrm{n}}>1 \mathrm{MeV}$.

The process of slowing down neutrons by scattering interactions is called moderation. Tabie 7.6 shows that the lighter elements are the mont efficient moderators, with hydrogen boing the best. This is illutrated further in Table 7.7 where the average number of collinions required to thermalize $1 \mathrm{MeV}$

\begin{tabular}{|c|c|}
\hline $\begin{array}{l}\text { Scattering } \\
\text { Nucleus }\end{array}$ & $\Delta E_{\max } / E_{n} \times 100$ \\
\hline $\begin{array}{l}\mathrm{H} \\
{ }^{2} \mathrm{H} \\
{ }^{4} \mathrm{He} \\
16 \mathrm{O} \\
{ }^{56} \mathrm{Fe} \\
{ }^{200} \mathrm{~Pb}_{\mathrm{Pb}} \\
{ }^{238} \mathrm{U}\end{array}$ & $\begin{array}{c}100 \\
89 \\
64 \\
22 \\
7 \\
2 \\
1.7\end{array}$ \\
\hline
\end{tabular}
neutrons is given for come common materials. It takes an order of magnitude fower collinions to thermalive in $\mathrm{H}_{2} \mathrm{O}$ than in heavier materiale. Hydrogenous

TRBLE 7.6

\section{MAXIMUM BNERGY LOSS FOR TARGET NUCLR (\%)}


compounds, e.g., $\mathrm{H}_{2} \mathrm{O}$ and $\mathrm{CH}_{2}$ in a sample will usually significantly influence the assory measurement. However, elastic scattering processes from heavier elements will have relatively little effect on the neutron count rate. This is due to two factors. First, the neutron detectors used for passive assay have relatively constant detection efficiency for neutron energies over a wide range, with no attempt made to distinguish ene energy neutron from another. Thus, the energy loss due to a scattering ovent will usually not prevent the neutron from being counted. The second factor is the large-angle scattering that a neutron can undergo with orly a small energy change. The values in Table 7.6 are for neutron scattering through $180^{\circ}$. Coneequently, although some neutrons are scattered awor from the detector, this may be compensated for by other neutrons being scattered into the detector. In fact, neutrons can be scattered into the detector or back into the sample by materials close to the counting setup, e.g., a concrete floor or wall.

Therefore we conclude that the calculation of sam. ple cttenuation is not straightforward. Using the total cross section will overpredict the sample attenuation.

\section{5.d. Multiplication Processes}

There are two kinds of neutron interactions that result in an increased number of free neutrons (multiplication). The first of these, the $(n, 2 n)$ reaction, occurs only above approximately $E_{p}=7 \mathrm{MeV}$, with the exception of interactions with ${ }^{\mathrm{B} e}$ and ${ }^{2} \mathrm{D}$. For these two isotopes the thresholds are $\sim 2.6$ and $\sim 3.2 \mathrm{MeV}$, respectively, with comparatively small cross sections. In most passive assay situations the $(n, 2 n)$ will nut contribute significantly.

The second type of multiplicative interaction is very important, namely, reutron-induced fission. Fissionable isotopes fall into two categories with respect to neutron-induced fission, tissile and fertile isotopes. The tissile isotopes are those which undergo fission for neutrons with energies down to thermal, whereas the fertile isotopes require the incoming neutron to have some minimum energy before tission will take place. The threshold energy varies from $\sim 500 \mathrm{keV}$ for ${ }^{240 \mathrm{Pu}}$ to $\sim 2 \mathrm{MeV}$ for ${ }^{232} \mathrm{Th}$. Figure 7.2 shows the fission cross section as a function of neutron energy for some of the isotopes.

As mentioned in Sec. 7.3, for a given atomic number $Z$, the fertile isotopes will have higher spontaneous fission rates than the fissile upecies. However, these emitted neutrons have a certain probability of interacting with the fissile material in the sample, causing additional fission neutrons to be emitted. Several factors influence the magnitude of the multiplication in a given sample.

1. Quantity and denaity of fisaionable material-the more material localized in the sample, the better the chance for interaction.

2. Presence of moderator-because the firsion cross section for the fissile species becomes as large $a_{B} 800 \mathrm{~b}$ at very low neutron energies, the presence of moderating material, particularly hydrogen, will increase the magnitude of the fission multiplication significantly.

3. Reflecting material in the vicinity of the sample-reflecting neutrons into the sample will give

TABLE 7.7

\section{NEUTRON MODERATION PARAMETERS FOR COMMON NDA MATERIAL}

\begin{tabular}{|c|c|c|c|}
\hline Material & $\begin{array}{l}\text { Density } \\
\left(\mathrm{g} / \mathrm{cm}^{3}\right) \\
\end{array}$ & $\begin{array}{l}\text { No. of Collisions } \\
\text { for Thermalizetion" }\end{array}$ & $\begin{array}{c}\text { Mean Free } \\
\text { Path-Thermal (cm) }\end{array}$ \\
\hline $\begin{array}{l}\mathrm{H}_{2} \mathrm{O} \\
\mathrm{C} \\
\mathrm{Al} \\
\mathrm{Zr} \\
\mathrm{Pb} \\
\mathrm{UO}_{2}\end{array}$ & $\begin{array}{c}1.00 \\
1.60 \\
2.70 \\
6.40 \\
11.35 \\
10.0\end{array}$ & $\begin{array}{r}18 \\
111 \\
242 \\
803 \\
1823 \\
486\end{array}$ & $\begin{array}{c}0.29 \\
2.60 \\
10.2 \\
2.89 \\
2.71 \\
1.85\end{array}$ \\
\hline
\end{tabular}

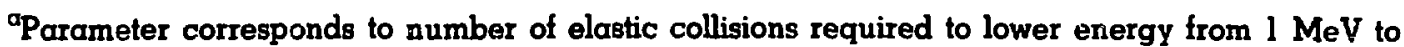
$0.025 \mathrm{eV}$ (thermal).

barameter correspond's to mean distance traveled between coilisions by a thermal-energy neutron.
} 


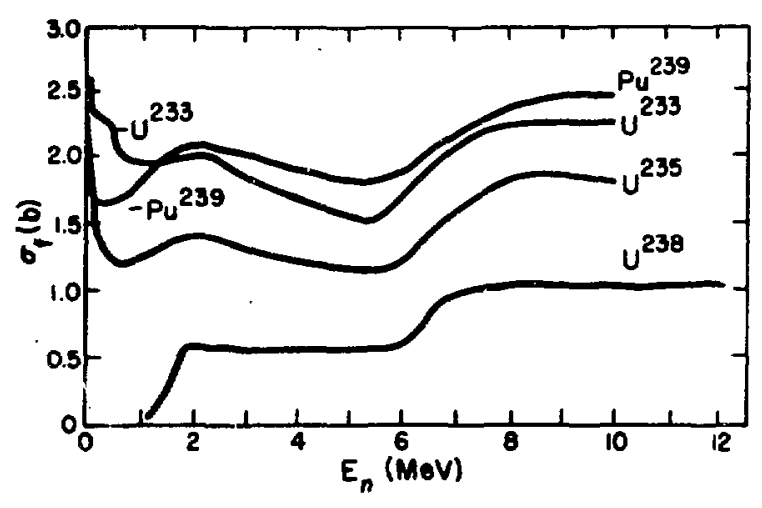

Fig. 7.2.

Neutron-induced fission cross section vs neutron energ.

the neutron another opportunity to cauve a fiecion, particularly if it comes back at a lower energy.

4. Shape of sample, in particular the ratio of surface area to volumo-in simpleat torms the greater the surface area the more likely a neutron will leak out before interacting.

Multiplicution and its effect on asery are diecuseed in more detail in Sec. 9.2.

\section{REFERENCES}

7.1. A. O. Hanson, in Fast Neutron Physics, J. B. Marion and J. L. Fowler, Eds. (Interscience Publithers, Inc., NY, 1960), Vol. 1, Chap. 1A.

7.2. H. Werle, "Spectrum Meanurements of Radioactive Neutron Sources in the $10 \mathrm{keV}-10 \mathrm{MeV}$ Energy Region with Proton-Recoil Counters," Rarlaruhe Nuclear Research Canter External Report INR-4170-25 (February 1970), Translation ORNL-tr2415.

7.3. E Amaldi, Encyclopedia of Physics (SpringerVerlag, Berlin 1959), Vol. 38, p. 119.

7.4. Brookhaven National Laboratory, "Neutron Cros Sections," Brookhaven National Laboratory Report BNL 325, Vol. I-III (MaY 1964 - Feb. 1965).

7.5. S. Glasstone and M. C. Edlund, The Elements of Nuclear Reactor Theory (Van Nostrand Company, NJ, 1952). This is an excellent reference book. Chapters 3 and 4 are of particular interest in that they deal with many of the subjects discused in this manual. 


\section{NEUTRON DETECTORS}

\subsection{Introduction}

There are two basic mechanisms by which a fast noutron can be detected. First, the neutron may collide with matter in a detecting medium and give up recoil energy to the medium, which is convertid either into light or electrical charge, the magnitude of which is a meanure of kinetic energy of the neutron. Secondly, if the medium contains isotopes with which the neutrons can enter into an exoergic nuclear reaction, the energy of this reaction can be converted into ionization, the presence of which indicates the interaction of a neutron with the detecting medium. For $\alpha$ comprehenoive discussion of all kinds of neutron detectors, the reader is referred to Chaps. II and III of Marion and Fowler ${ }^{8.1}$ and Chap. 10 of Price. ${ }^{8.2}$

When no information is desixed about the energy of detected neutrons, and sensitivity is needed over a broad neutron energy r'inge, the simplest, most stable type of fast-neutron detector system is one in which the neutrons are first thermalized by moderation in a hydrogen-containing medium such as polyethylene, paraffin, or water; and the thermal neutrons are detected using either the ${ }^{10} \mathrm{~B}(\mathrm{n}, \alpha)^{7} \mathrm{~L}$ i reaction or the ${ }^{\prime} \mathrm{He}(n, p)^{3} \mathrm{H}$ reaction. The large thermal-neutron cross sections for these two reactions ( $3837 \mathrm{~b}$ for the former, $5327 \mathrm{~b}$ for the latter) make possible the construction of very efficient detectors. Normally, the thermal-neutron detectors are gas proportional counter tubes in which the energetic charged particles, which result from the reactions, dissipate their energy by ionizing the gas in the counter tube. The liberated electric charge is collected and amplified to generate a signal.

\subsection{Gas Proportional Counter Tubes}

One of the oldeat devic.se employed for nuclear radiation detection is a gas-filled chamber with electrodes arranged to collect the electicic charge produced by the interaction of nuclear radiation with the chamber gas. The chamber is usually a metal cylinder with a thin, insulated wire mounted on its axis (Fig. 8.1). The center wire, or anode, is maintained at $\alpha$ positive potential of from several hundred to several thousand volts with respect to the outer cylinder, or cathode, which also serves as the container for the counter gas.

Nuclear radiation interacts with the counter gas in a number of ways. Gamma radiation producen energetic tree electrons by Compton scattering, photoelectric effect, or pair production. The free electrons so produced are slowed down by ionining collisions with counter gas molecules, and a tree charge $\mathbf{Q}=\mathrm{E} e / \varphi$ is produced where $\mathrm{E}$ is the initial photon energy, $\theta$ is the electronic charge, and $\varphi$ is the average energy necessary to produce an ion pair. A beta particle energetic onough to penetrate the chamber wall (or originating from a source placed within the counter) will interact directly with the counter gas to produce $\alpha$ track of ion pairs. Heavier charged particles, such as alphas, likewise lose energy by ionizing gas molecules in their path. However neutrons having no charge will not produce ionization along their path through matter. A neutron loses energy by colliding with an atomic nucleus, to which it gives up part of its energy by momentum transfer. This is the scattering reaction discuesed in Sec. 7.5.c. The recoiling atom then ionizes and gives up the energy it has gained by ionizing other molecules. For the inomentum-tronster process to be most effective, the atom against which the neutron recoils should be hydrogen, in which case the neutron can transfer up to all of its energy to the recoiling proton (see Table 7.6).

Anciher way of detecting a neutron is to have it cause an exoergic (energy-reloasing) nuclear reaction, the products of which are charged particles. Such a reaction can occur when a neutron react with ${ }^{10} \mathrm{~B}$.

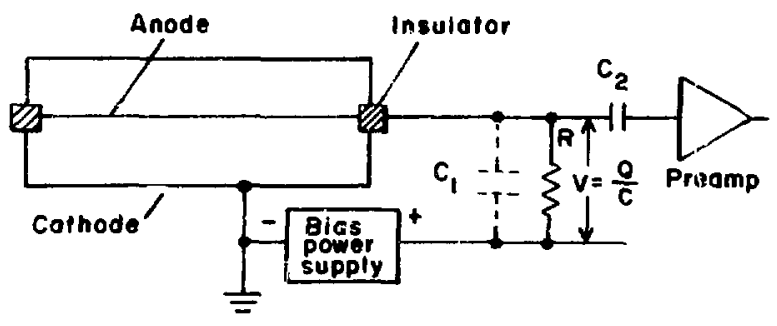

Fig. 8.I.

Tipical proporional counter setup. 


$$
{ }^{10} \mathrm{~B}+\mathrm{n} \rightarrow{ }^{7} \mathrm{Li}+{ }^{4} \mathrm{He}+2.78 \mathrm{MeV}
$$

in which case the $2.78 \mathrm{MeV}$ is shared by the product ${ }^{7} \mathrm{Li}$ and ${ }^{4} \mathrm{He}$, or

$$
\begin{array}{r}
{ }^{10} \mathrm{~B}+\mathrm{n} \rightarrow{ }^{7} \mathrm{Li}^{*}+{ }^{4} \mathrm{He}+2.30 \mathrm{MeV} \\
{ }^{7}{ }_{\mathrm{Li}}{ }^{*} \rightarrow{ }^{7} \mathrm{Li}+0.48 \mathrm{MeV}
\end{array}
$$

in which latter case the gamma-ray energy is usually lost from the detector.

An alternate nuclear reaction that can be used for detecting neutrons in a gas counter uses ${ }^{3} \mathrm{He}$.

$$
{ }^{3} \mathrm{He}+\mathrm{n} \rightarrow{ }^{3} \mathrm{H}+{ }^{1} \mathrm{H}+765 \mathrm{keV}
$$

which is then converted to energy of ionization as before.

What happens to the ion pairs generated within the detector volume depends upon the voltage applied between the cathode and anode. At low voltages the primary ions and electrons have a chance to recombine before they are collected at the

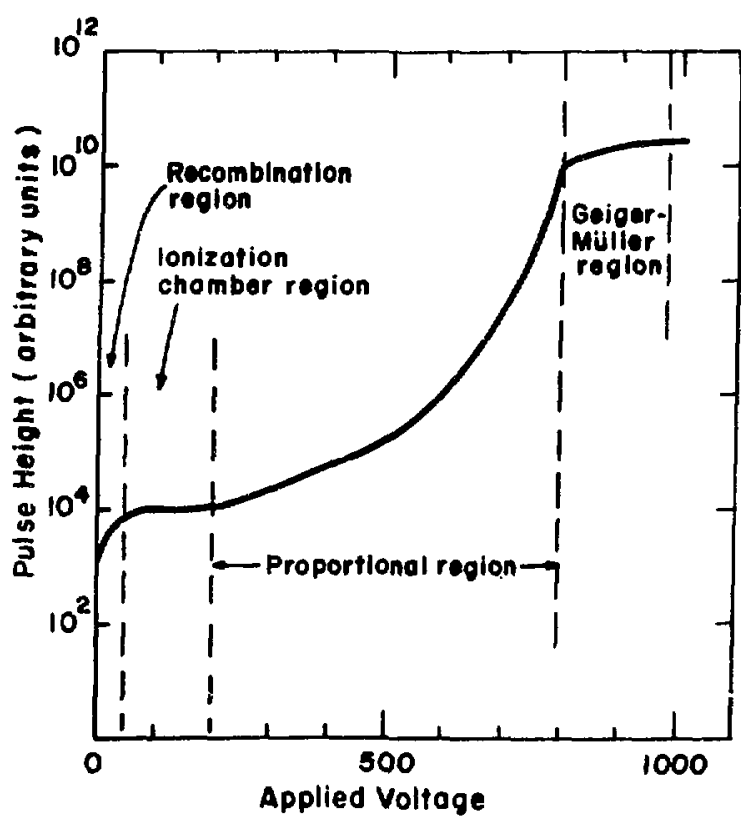

Fig. 8.2.

Fulse-height us applied-voltage curves to illustrate ionization, proportional, and Geiger-Muller regions of operation. walls or center wire of the detector. As voltage is increased (Fig. 8.2), a point is reached where all of the ion pairs are collected, and at this point $\alpha$ plateau is reached where further voltage increase produces no more ion pairs. In this state, the tube is said to be an ionization chamber. The charge collected at the anode is proportional to the energy deposited within the detector and is independent of the cnode voltcge.

As voltage is increased further, however, $a$ point is reached where primary ions in the chamber can be accelerated between intermolecular collisions enough so that they can ionize other molecules. The result is a multiplication process in which the total charge sollected on the anode is still dependent directly upon the initial energy deposited in the detector, but also increases rapidly with applied voltage. This is the so-called proportional-counter mode of detector operation. Charge multiplication of $10^{6}$ or more is possible before the counter loses its ability to distinguish between events of difforant energies.

As the voltage is increased even more, a point is reached where the charge collected is independent of the quantity of ionization initiating the event. The size of the pulse from the tube is characteristic only of the tube geometry and filling and of the external electronic circuitry. The detector is then a GeigerMuller tube. Geiger tubes are used in most low-level survey instruments and in applications where it is not necessary to determine the type or energy spectrum of the radiation being counted. The amplification available in the tube and the uniform pulse heights for detected radiation make the Geiger-Muller tube one of the easiest nuclear radiation detectors to use.

Neutrons are almost always accompanied by gamma radiation. Therefore, it is usually desirable that a neutron detector be capable of distinguishing between a neutron and a gamma ray. For this reason, gaseous neutron detectors are usually operated in the proportional region (in rare cases, in the ionization-chamber ragior).

If it is not necessary to know the energy of the neutron, it is expedient to slow down or moderate the neutron before detecting it to take advantage of the large thermal cross section of either the ${ }^{10} \mathrm{~b}(n, \alpha)^{7} \mathrm{Li}$ reaction or the ${ }^{3} \mathrm{He}(n, p)^{3} \mathrm{H}$ reaction. Normally, the boron is in the form of $\mathrm{BF}_{3}$ gas; it can also be introduced as a thin layer on the inside wall of the detector. If ${ }^{3} \mathrm{He}$ gas is used, it is usually mixed with a heavy gas such as argon or krypton to assure that the protons released in the ${ }^{3} \mathrm{He}(n, p)$ reaction are stopped before they reach the counter wall.

A puloe-height distribution from thermal neutrons incident upon typical ${ }^{3} \mathrm{He}$ proportional counters is shown in Fig. 8.3. The full-energy peak corresponds 
to events where the reaction products are stopped completely in the active region of the counter gas. The spread of the full-energy peak is due to the statistical nature of the ionization process and to the unevenness of the accelerating field over the volume of the tube. There is a low-energy tail of pulses due to neutrons that react near the walls or field-free "dead spaces" at the ends of the tubes. For these events, some of the reaction product energy has been dissipated in the tube wall or end spaces and has therefore not been converted into ion pairs. Finally, at low pulse heights the response to gamma radiation is seen. Because the electrons released by photon interactions have much greater track lengths than the ions created by neutron reactions, there is an upper limit (around 300 to $500 \mathrm{keV}$ ) to the energy which can be deposited in this type of counter by a Compton or photoelectron. However, if the gamma field is intense, it is possible for pulses due to gamma radiation to overlap and to give "pileup" pulses, which will overlap part of the neutron pulse distribution.

It is possible to discriminate between neutron and gamma pulses in these detectors. Because the track lengths of Compton electrons are longer than those of neutron reaction products, the collection of ion pairs in an electron track will usually spread over a greater time interval than will the collection of ion pairs produced in a proton, alpha, or triton track with the same total energy. Therefore, the risetimes of counter pulses arising from gamma radiation are generally longer than those from neutrons. Circuitry exists which uses this pinciple to reduce the gamma pileup effect in neutron proportional counters. Where gamma fields are high, this techniqus is sometimes helpful.

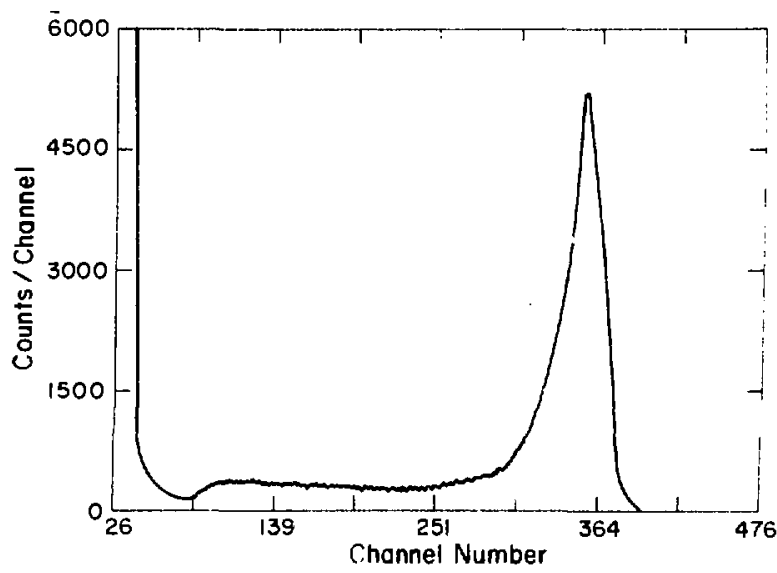

Fig. 8.3.

Differentist pulse-height distribution from thermal neutrons in a ${ }^{3} \mathrm{He}$-filled proportional counter tube.

\subsection{Moderator Assemblies}

Thermalizaiion of fast neutrons before detection serves two purposes: it increases detection efficiency and reduces energy dependence of the overall detection system efficiency. As discussed in Chap. 7, a detection efficiency independent of energy tends to make the assiny independent of matrix effects due to scattering in the sample. One of the oldest applications of this principle is the "Long Counter" of Hanson and McKibben. ${ }^{8.3}$ This counter, consisting of a $\mathrm{BF}_{3}$ tube imbedded axially in a paraffin cylinder, 20 -cm-diam by $30-\mathrm{cm}$-long, has a response to neutrons which is energy independent from $25 \mathrm{keV}$ to $5 \mathrm{MeV}$ to within $15 \%$. Improved versions of this counter have been made employing shielding to achieve directional sensitivity, with some holes drilled in the front detector wall to improve the flatness of response at low energies. Allen ${ }^{8.4}$ discusses the design of these counters in detcil.

Two more efficient and flatter response counters were developed by East and Walton ${ }^{8.5}$ to implement neutron-counting techniques being developed for nondestructive assay of fissionable materinls. One of these, similar in size and shape to the long counter, employs five ${ }^{3} \mathrm{He}$ proportional counter tubes and is $11.5 \%$ efficient (intrinsic) over the $23-\mathrm{cm}$-diam sensitive region and is flat to within $\pm 3 \%$ from $25 \mathrm{keV}$ to $5 \mathrm{MeV}$. The other is a 50- by $61-$ by $15-\mathrm{cm}$ polyethylene slab containing $13^{3} \mathrm{He}$ counters with an intrinsic efficiency of $18 \%$. This "slab detector" is shown in Fig. 3.4.

\subsection{Portable Neutron Probe, "SNAP"}

A shielded neutron assay probe (SNAP) ${ }^{8.6}$ has been designed to use with commercially available portable electronic units, e.g., Eberline SAM I and Ludlum Model 22. The SNAP detector consists of two (or one) 2-cm-diam by 14-cm-long (active), 4-atm ${ }^{3} \mathrm{He}$ counters contained in a 7.6-cm-diam, 23-cmlong polyethylene cylinder surrounded by a cadmium sheet. This probe may be used without shielding, or be placed inside a directional shield, as shown in Fig. 8.5. The weight of SNAP, including shield, is approximately $9 \mathrm{~kg}(20 \mathrm{lb})$, which is adequately portable. Figure 8.6 shows a SNAP system complete with a portable electronic unit.

The total detection efficiency of SNAP (with its shield) for a point neutron source $1 \mathrm{~m}$ from the detector is given in Table 8.1 and Fig. 8.7. Corresponding efficiency values for the slab detector shown in Fig. 8.4 are included for comparison. However, the weight of the slab detector with shield is greater then $90 \mathrm{~kg}(200 \mathrm{lb})$, hardly portable. Because the SNAP is 

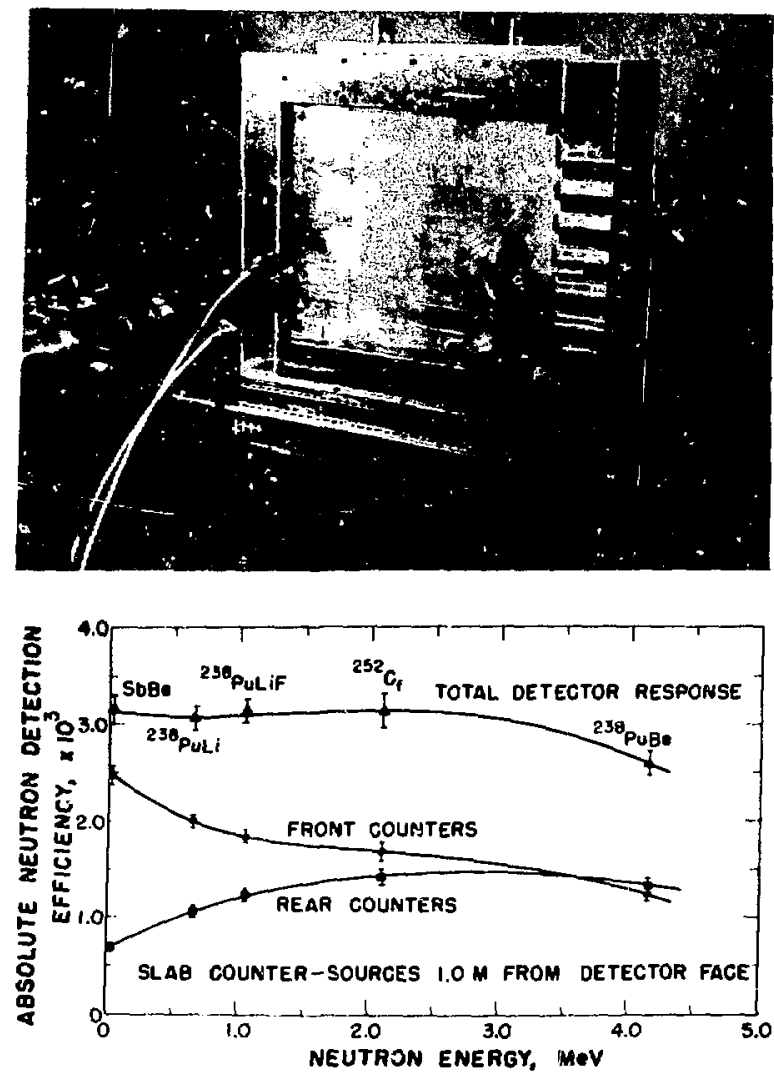

Fig. 8.4

High efficiency slab neutron delector.

not tlat in energy response, it will have a slightly enhanced sensitivity to sample moderation.

As a function of radial distance $\mathrm{I}$ from the axis of the detector, the efficiency has been found to vary as $(r+2.54)^{-2}$, where $x$ is measured in centimeters and $r$ $\geq 13 \mathrm{~cm}$. When performing measurements in close proximity to surfaces that are highly reflective to fast neutrons (such as concrete), the efficiency will decrease less strongly than $(x+2.54)^{-2}$. The directional sensitivity of SNAP, measured with a ${ }^{238} \mathrm{PuLi}$ source as a function of angle around the detector, is presented in Fig. 8.8. SNAP provides approximately $120^{\circ}$ of sensitivity in the forward direction and exhibits a shielding factor of greater than 4.2 for background nentrons impinging at angles between 80 and $280^{\circ}$. For source neutrons of higher mean energy than $650 \mathrm{keV}$, the shielding factor is somewhat less; for example, it is about 2.25 for ${ }^{252} \mathrm{Cf}$ source neutrons.

Current applications involving SNAP include verification of UF 6 cylinder contents, UF 4 "green" salt, and metallic plutonium buttons.
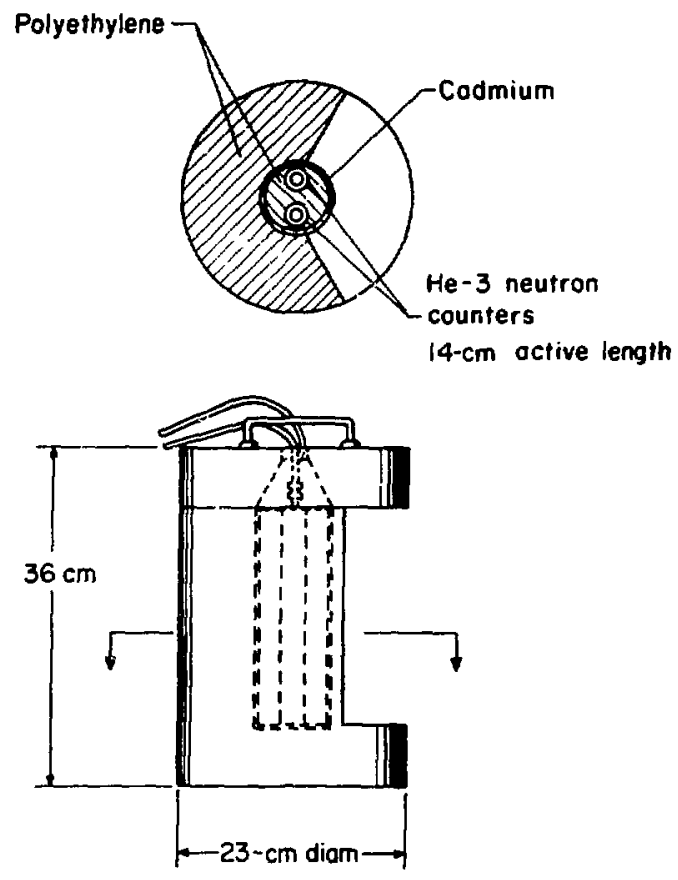

Fig. 8.5.

Assembly drawing of the portable shielded neutron assay' probe (SNAP).

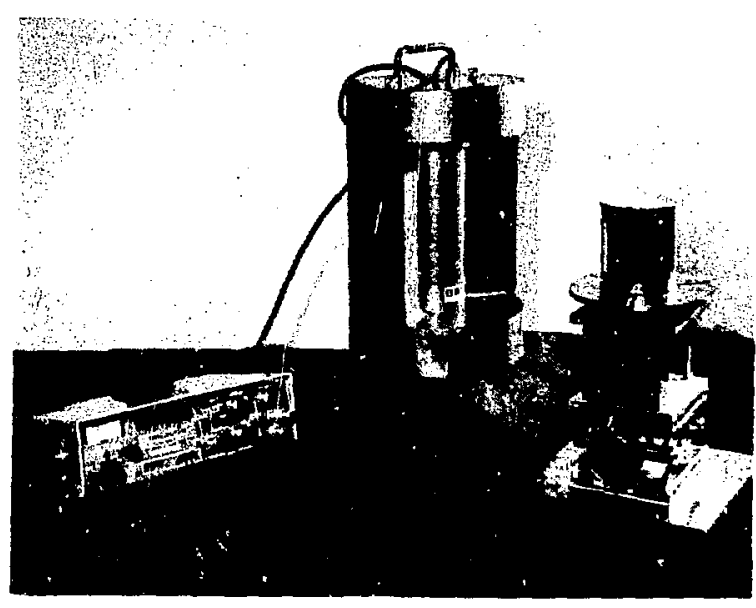

Fig. 8.6.

A porsable shielded neusron assay probe (SNAP), complete with electronics.

\subsection{Other Neutron Detectors}

1. Plastic and Liquid Scintillators. Plastic and liquid scintillators are sometimes used for the detection and energy measurement of fast neutrons. The 
TABLE 8.1

\section{TOTAL EFFICIENCY FOR POINT SOIJRCE AT ONE MERER}

\begin{tabular}{|c|c|c|c|}
\hline Source & $\begin{array}{c}\bar{E}_{\mathfrak{n}} \\
\left(\mathrm{MeV}_{\mathrm{e}}\right)\end{array}$ & SNAP & Slab Detector \\
\hline $\begin{array}{l}{ }^{238} \mathrm{PuLi} \\
{ }^{238} \mathrm{PuLiF} \\
{ }^{252} \mathrm{Cf}\end{array}$ & $\begin{array}{l}0.65 \\
1.1 \\
2.2\end{array}$ & $\begin{array}{l}9.7 \times 10^{-5} \\
8.3 \times 10^{-5} \\
6.6 \times 10^{-5}\end{array}$ & $\begin{array}{l}3.1 \times 10^{-3} \\
3.1 \times 10^{-3} \\
3.1 \times 10^{-3}\end{array}$ \\
\hline
\end{tabular}

aTotal Efficiency $\left(\frac{\text { Neutrons Detected }}{\text { Neutrons Emitted }}\right)$

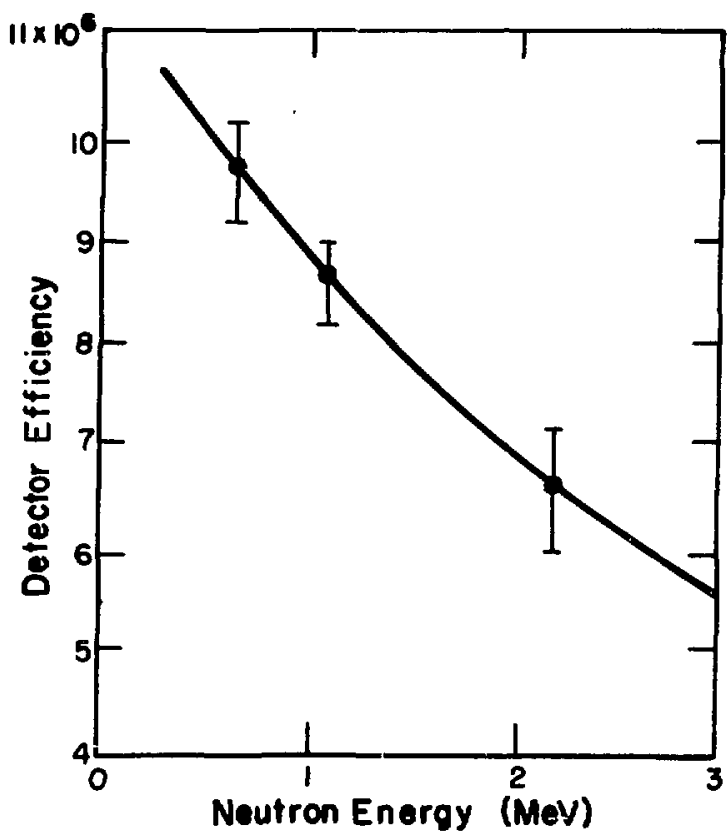

Fig. 8.7.

Efficiency vs neutron enterg.' for SNAP detector.

detection mechanism is the recoil of hydrogen atoms in the scintillator and subsequent conversion of the recoil energy into pulses of light, which are detected by a photomultiplier tube. Plastic and liquid scintillators have the advantage that, although the risetime of proportional counter signalo is of the order of a few microseconds, the scintillators have risetimes in the nanosecond range. Thus, scintillator eystems can be operated at much greater counting rates without pileup or deadtime problems.

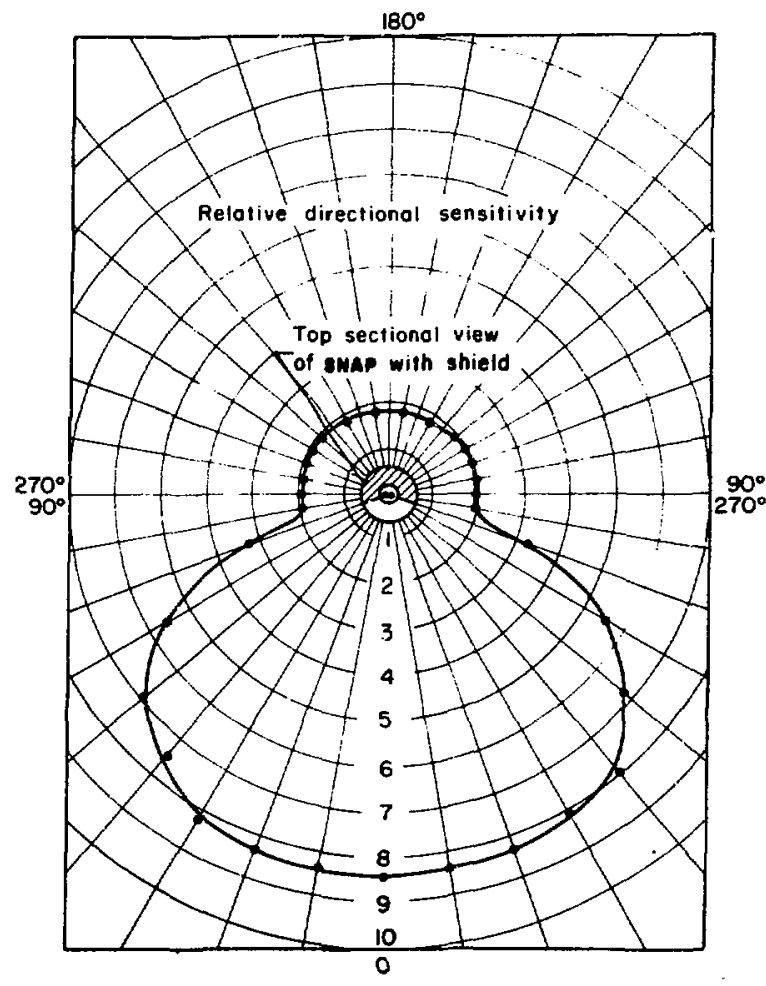

Fig. 8.8.

Directional sensitivity of SNAP as a function of angle, as measured in the midplane of the detector with a PuLi point source $\left(E_{n} \simeq 0.65 \mathrm{MeV}\right)$.

A further advantage accrues when contrasting plastic-scintillator neutron coincidence counting systems with the more frequently used (for safeguards) thermalixing coincidence systems with proportional counters. Because the average neutron lifetime in detectors of the latter type is between 15 and $100 \mu \mathrm{s}$, very long coincidence gates must be used if the systems are to be efficient.

The weakness of plastic or liquid recoil scintillators is their sensitivity to gamma radiation. Pulses from gamma rays and from neutrons are approximately the same size in these detectors. Differences do exist, however, between the shapes of neutron- and gamma-ray-induced pulses from some liquid scintillators. These differences have been used to make such detectors less gamma sensitive. B.7 $^{2}$

2. Hornyak Buttons. ${ }^{8.8}$ A Lucite block impregnated with silver-activated zinc oulphide will scintillate when irradiated with fort neutrons. The principal means for detection is recoil of the hydrogen in the Lucite; however, the ${ }^{32} S(n, p){ }^{32} p$ and ${ }^{32} S(n, \alpha)^{29} S_{i}$ reactions also contribute. These detectors have a 
maximum efficiency for $5 . \mathrm{MeV}$ neutrons. ${ }^{8.9} \mathrm{Gamma-}$ ray discrimination is available on the basis of pulseheight differences.

3. "He and Proton-Recoil Proportional Counters. For fast-neutron detection without previous thermalization, recoil of neutrons from light nuclei in proportional counters is used as a means of detection and energy measurement. Techniques for neutron spectroscopy with proton-recoil counting have been developed and used by Bennett et al. 8.10 The ${ }^{4} \mathrm{He}$ counter is used in numerous safeguards active assay systems. This latter type of counter is relatively insensitive to gamma radiation and lowenergy neutrons and is used for detection of fast neutrons from fission in the presence of thermal neutrons inducing fission.

\section{REFERENCES}

B.1. J. B. Marion and J. L. Fowler, "Fast Neutron Physics, Pout I," (Interscience Publishers, NY, 1960).

8.2. W. J. Price, "Nuclear Radiation Detection," (McGraw-Hill Inc., NY, 1958).

8.3. A. O. Hanson and J. L. McKibbon, "A Neutron Detector Having Uniform Sensitivity from $10 \mathrm{keV}$ to 3 MeV," Phys. Ruv. 72, 673 (1947).
8.4. W. D. Allen, Chap. III.A of Ref. 8.1.

8.5 L. V. East and R. B. Walton, "Polyethylene Moderated ${ }^{3} \mathrm{He}$ Neutron Detectors," Nucl. Instrum. Methods 72, 161 (1969).

8.6. R. B. Walton and T. L. Atwell, Los Alamos Scientific Laboratory A-1 Progress Report LA-5291PR (1973), p. 14. The title of this article is "Portable Neutron Probe, 'SNAP'."

8.7. R. A. Winyard, I. E. Lutkin, and G. W. McBeth, "Pulse Shape Discrimination in Inorganic and Crganic Scintillators.I," Nucl. Instrum. Methods 95, 141 (1971).

B.8. W. F. Hornyak, "A Fast Neutron Detector," Rev. Sci. Instrum. 23, 264 (1952).

8.9. C. O. Muilhause, pp. 4 55-407 of Ref. 8.1.

8.10. E. F. Bennett and T. J. Yale, "Techniques and Analyses of Fast Reactor Neutron Spectroscopy with Proton-Recoil Proportional Counters," Argonne National Laboratory report ANL-7763 (1973). 


\section{PASSIVE NEUTRON VERIFICATION MERSUREMENTS}

\subsection{Considerations in Applying Passive Neutzon Counting}

In approaching an asscy problem for which passive neutron counting will be used as the signature, the first step is to culculate the contribution of the various isotopes and neutron processes to the total count rate. To do this, information on the chemical form and isotopic composition is noeded. Consider the tollowing ihree chemical forms of recycle plutonium: motal, $\mathrm{PuO}_{2}$, and $\mathrm{PuF} 4$. Table 9.1 lists the individual nentron ominsion rates for each isotope and procem. The rates were computed from the values given in Tables 7.2, 7.3, and 7.4. The spontaneous tission rute, being indepandent of chemical torm, is the same in each case whereas the $\{\alpha, n)$ rate changes dramatically. General conclusions that can be reached for this particular plutonium isotopic compotition are

1. For metal having no $(\alpha, n)$ component, the quantity being measured is $210 \mathrm{Pu}$ (with contributions from ${ }^{233} \mathrm{Pu}$ and ${ }^{24} \mathrm{Pu}$ ). This will be true for almost any ${ }^{240} \mathrm{Pu}$ isotopic percentage found in the nucloar fuel cycle. It will not be true for material rich in ${ }^{238} \mathrm{Pu}$ such as is used in the heart pacer program.
2. The neutron output from $\mathrm{PuO}_{2}$ has comparable contributions from both processes, $107 \mathrm{n} / \mathrm{g}-\mathrm{s}$ from $(x, \mathrm{n})$ and $210 \mathrm{n} / \mathrm{g}-\mathrm{s}$ from spontaneous fission. The small amount of ${ }^{236} \mathrm{Pu}(0.3 \%)$ contributes $14 \%$ of the total output.

3. For $\mathrm{PuF}_{4}$, the neutron rate is dominated by the $(\alpha, n)$ prccesses and therefore is more sensitive to the amount of the minor ${ }^{238} \mathrm{Pu}$ isotope. It is interenting to note that, in this situation, neutron counting gives the same information as calorimetry, namely, the $\alpha$ emis. sion rate.

The above examples do not include the contribution from $211_{\mathrm{Am}}$ that will increase on $2{ }^{2} \mathrm{Pu}$ decars. Three to four years aftor chomical esparation, the $21_{\mathrm{Am}}(a, n)$ rate will bo comparable to that of ${ }^{20 P_{\mathrm{u}}}$ and ${ }^{210} \mathrm{Pu}$ for this recyclo material.

Light elomont impuritien, particularly beryllium, can affect the total neutenn roto. Consult Table 7.1 for the specific eloment of pomible concern.

Once the eminion rate have been calculated, an entimate of the counting rate in the defector can be made. The counting rote is the omimion rate times the total detection afficiency (Sec. 2.4.b). Convider counting the plutonium samples described above using the SNAP probe at a exmple-10-detector dintance of $30 \mathrm{~cm}$. The overall efficioncy is

TABLE 9.1

NEUTRON DMISSION RATES FOR Pu METAL, PuO 2 AND PuF4

\begin{tabular}{|c|c|c|c|c|}
\hline \multirow[b]{2}{*}{ Isotope } & \multirow[b]{2}{*}{ Wt\% } & \multicolumn{3}{|c|}{ 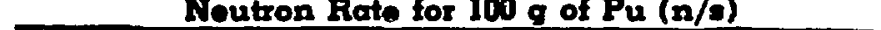 } \\
\hline & & $\begin{array}{c}\text { Motal } \\
\text { (Spontenoous rission) }\end{array}$ & $\begin{array}{l}\mathrm{PuO}_{2} \\
(a, n) \\
\end{array}$ & $\begin{array}{l}\text { PuF: } \\
(\alpha, n)\end{array}$ \\
\hline $\begin{array}{l}{ }^{238} \mathrm{Pu} \\
{ }^{239} \mathrm{Pu} \\
{ }^{240} \mathrm{Pu} \\
{ }^{241} \mathrm{Pu} \\
{ }^{242} \mathrm{Pu}\end{array}$ & $\begin{array}{r}0.3 \\
75.6 \\
18.0 \\
5.0 \\
1.1\end{array}$ & $\begin{array}{r}746 \\
2 \\
18400 \\
\sim 0 \\
1900\end{array}$ & $\begin{array}{r}4200 \\
3400 \\
3060 \\
50 \\
10\end{array}$ & $\begin{array}{r}630000 \\
325000 \\
288000 \\
5000 \\
1000\end{array}$ \\
\hline & Total & 21048 & 10720 & 1219000 \\
\hline
\end{tabular}




$$
\begin{aligned}
\varepsilon & \left.=6.6 \times 10^{-5}\right)\left(\frac{102.54}{32.54}\right)^{2} \\
& =6.6 \times 10^{-4} \text { counts/neutron emitted } .
\end{aligned}
$$

This efficiency was obtained by correcting the value given in Sec. 8.4 for a cloner sample-dotector separation $\left[(r+2.54)^{-2}\right.$ dependence $]$. Counting rates for the three 100-g plutonium scmples are plutonium metal, 13.9 counte/s; $\mathrm{PuO}_{2}, 21.0$ counts/s; and $\mathrm{PuF}_{4}$, 838 counts/o.

These rates must now bo compared to the background rates expected at the counting location. Knowledge of backgrounds can anly be obtained by experience and direct measurenent. The SNAP detector is designed to preferentially detect neutrons striking its front face. Backgrounds wili be reduced if the detector is oriented with the front face away from the major sources of background, such as storage vaults.

With the exception of hydrogen (e.g., $\mathrm{H}_{2} \mathrm{O}$ and $\mathrm{CH}_{2}$ ), matrix materials commonly found in aseay samples do not greatly affect fant-nautron flux. To illustrate the offect that matrix material has on neutron counting, the following experimeat was performed using the SNAP detector (Sec. 8.4). A 4-lifer can was placed $30 \mathrm{~cm}$ from the front face of the SNAP unit with provision for positioning a neutron source in the center of the can. The counting rate of the source was measured for the empty can. Matrix material was added to the can and the rate was remeasured. Table 9.2 presents the results of these measurement normalized to the empty can case. An examination of column 5 indicates that most of the outgoing source neutrons do, in these examples, interact with the matrix material before emerging from the can. The dominant interaction is by elastic scattering with varying amounts of energy loes depending on the particular scatiering material. Because the scattering material is located symmetrically around the neutron source, the number of neutrons scattered away from the detector is balanced by neutrons scattered into the SNAP, that is, inscattering equals outscattering. The counting rate is greater with the material present because the detector efficiency is higher at lower neutron energies (Fig. 8.7).

For the hydrogenous material, the count rate insreases it the loweat denrity, but as more hydrogen is added the rate decreases. With the higher density sample, a greater number of the neutrons are moderated to the point where they are absorbed before they reach the detector tubes. They can be absorbed by the cadmium cover of the SNAP unit or by the hydrogen, either in the sample or in the detecto:. The SNAP detector is said to be undermoderated.

The SNAP detector was denigned for a specitic situation in which the matrix material did not vary. It is possiblo to modify the SNAP detector dexign to make it efficiency lese energy dependent with a small increase in weight. But oven as presently

TRBLE 9.2

MATRIX EFFECTS ON NEUTRON COUNTING

\begin{tabular}{|c|c|c|c|c|c|}
\hline \multirow[b]{2}{*}{ Material } & \multicolumn{2}{|c|}{ Weight } & \multirow{2}{*}{$\begin{array}{l}\text { Density } \\
\left(\mathrm{g} / \mathrm{cm}^{3}\right)\end{array}$} & \multirow{2}{*}{$\begin{array}{c}\text { Interaction } \\
\text { Probability } \\
\text { (\%) }\end{array}$} & \multirow{2}{*}{$\begin{array}{c}\begin{array}{c}\text { Normalized } \\
\text { Counting } \\
\text { Rate }\end{array} \\
\end{array}$} \\
\hline & $\mathrm{kg}$ & $\underline{\mathbf{l b}}$ & & & \\
\hline $\begin{array}{c}\text { Emply } \\
\mathbf{P b} \\
\mathrm{Fe} \\
\mathrm{SiO}_{2}\end{array}$ & $\begin{array}{r}0 \\
26 \\
17 \\
6\end{array}$ & $\begin{array}{r}0 \\
58 \\
37 \\
13\end{array}$ & $\begin{array}{l}0 \\
6.9 \\
4.4 \\
i .6\end{array}$ & $\begin{array}{r}0 \\
57 \\
63 \\
80\end{array}$ & $\begin{array}{l}1.00^{b} \\
1.06 \\
1.10 \\
1.12\end{array}$ \\
\hline $\begin{array}{c}\mathrm{CH}_{2} \\
\mathrm{CH}_{2} \\
\mathrm{H}_{2} \mathrm{O}\end{array}$ & $\begin{array}{l}0.7 \\
2.5 \\
3.6\end{array}$ & $\begin{array}{l}1.6 \\
5.6 \\
8\end{array}$ & $\begin{array}{l}0.2 \\
0.7 \\
1.0\end{array}$ & $\begin{array}{l}60 \\
35 \\
98\end{array}$ & $\begin{array}{l}1.21 \\
1.06 \\
0.73\end{array}$ \\
\hline
\end{tabular}

( $\sim$-liter container $16.5-\mathrm{cm}$-diam by $19-\mathrm{cm}-\mathrm{high}$ ) 
TABLE 9.3

\section{MULTIPLICATION OF PU FOR VAPIOUS SHAPES}

(Pu Weight-2 kg; $10 \%{ }^{24\left(P_{u}\right)}$

\begin{tabular}{|c|c|c|}
\hline Shape & $\begin{array}{l}\text { Dimensions } \\
(\mathrm{cm})\end{array}$ & $\frac{\text { Total Neutyone Emitted from Sample }}{\text { No. of Spontanoous Fission Neutrons }}$ \\
\hline $\begin{array}{l}\text { Sphere } \\
\text { Cube } \\
\text { Cylinder } \\
\text { Cylinder } \\
\text { "Pancake" }\end{array}$ & $\begin{array}{l}\mathrm{r}=2.98 \\
\mathrm{Q}=4.81 \\
\mathrm{~d}=5.21, \mathrm{~h}=5.21 \\
\mathrm{~d}=7.07, \mathrm{~b}=2.83 \\
\mathrm{~d}=8.91, \mathrm{~b}=1.78\end{array}$ & $\begin{array}{l}1.80 \\
1.73 \\
1.74 \\
1.63 \\
1.47\end{array}$ \\
\hline
\end{tabular}

designed, the sensitivity to matrix matorial is relatively sinall. Twenty-six kilograms of lead changand the counting rate by only $6 \%$.

The discussion in Sec. $\mathbf{S . 2}$ of count rate sensitivity to the location of nuclear material within the sample container is also applicable to neutron counting. The recommendations for sample-to-detector distances combined with sample rotation should be followed.

The type of calculations presented above provides guidance for planning the asear measurement. However, as was recommended earlier, the use of standard samples matching the unknown samples will lead to more sccurate assay values.

\subsection{Multiplication}

Many situations for which passive neutron counting is applicable involve large quantities of dense fissionable material, such as plutonium buttons. In these cases, the observed neutron emission rate from a sample can be increased when the initiol $(\alpha, n)$ and spontaneous fission neutrons induce additional fission reactions before passing out of the sample. In addition, the neutrons that have excaped from the sample can reflect back into the sample, usually after thermalization, and incluce additional fission reactions. Beccusa the neutron fission reaction releases more neutrons than it uses, there is a net gain or multiplication in the neutron emission rate. The magnitude of this multiplicaiion is very dependent on the mass, density, and spatial distribution of fistionable material in the sample. For example, Table 9.3 lists the multiplication for $2 \mathrm{~kg}$ of plutonium metal in different shapes: sphere, cylinder, "pancake," and cube. It can be seen that the multiplication eifect is large and is sensitive to the shape of the sample. Where possible, standards spanning the mass range of the unknowns should be used to cor- rect for multiplication as, well as for other matrix effects.

An article by Atwell at al. 9.2 is recommended as a detailed example of neutron counting applications, including correctione for multiplication, to the verification of plutonium metal buttons.

\subsection{Neutron Counting of Uranium Samples}

Total neutron counting of uranium samples is also fecrsible although usually larger quantities ore re. quired. The primary neutron source for some uranium common materials are given here.

1. Low enrichment $\mathrm{UO}_{2}$-neutron yield dominated by spontaneous fission in ${ }^{238} \mathrm{U}_{\text {; therefore gross }}$ counts nominally proportional to ${ }^{238} \mathrm{~J}$ weight.

2. High enrichment $\mathrm{UO}_{2}$-neutron yield dominated by ${ }^{234} U$ alphe decay reacting with the ${ }^{18} \mathrm{O}$ ( $0.20 \%$ abundant in normal oxygen).

3. UF6 -all enrichments-neutron yield dominated by ${ }^{234} U$ alpha decay reacting with fluorine.

Passive neutron counting can be used for the ${ }^{235} U$ assar of $\mathrm{UF}_{\mathrm{S}}$ cylinders if the ${ }^{235} \mathrm{U} /{ }^{234} \mathrm{U}$ ratio is knuwn. Because ${ }^{234} \mathrm{U}$ is the dominant alpha emitter in enriched uranium, it is the principal source of neutrons from ${ }^{19} \mathrm{~F}(\alpha, n)$ neutrons in $\mathrm{UF}_{\bar{b}}$. The ${ }^{235} \mathrm{U} /{ }^{234} \mathrm{U}$ ratio may vary by as much as a factor of two over the complete range of enrichment; but for low enriched uranium ( $<5 \%$ ), it is more nearly constant and verification measurements of limited accuracy may be possible without specific ${ }^{234} U$ isolopic data. 9.3

Table 9.4 lists alpha activities and neutron yields of the uranium isotopes. These data were used to 


\section{ALPHA-DECAY AND UF 6 NEUTRON YIELDS FOR U ISOTOPES}

\begin{tabular}{|c|c|c|c|c|}
\hline Isotope & $r_{1 / 2}(y x)$ & $\begin{array}{c}\text { Alpha } \\
\text { Activity } \\
(\alpha / g) \\
\end{array}$ & $f_{\alpha}^{\alpha}$ & Neutrons/g-5 \\
\hline $\begin{array}{l}{ }^{234} \mathrm{U} \\
{ }^{235} \mathrm{U} \\
{ }^{236} \mathrm{U} \\
{ }^{238} \mathrm{U}\end{array}$ & $\begin{array}{l}2.48 \times 10^{5} \\
7.13 \times 10^{8} \\
2.39 \times 10^{7} \\
4.51 \times 10^{9}\end{array}$ & $\begin{array}{l}2.27 \times 10^{8} \\
7.90 \times 10^{4} \\
2.35 \times 10^{6} \\
1.23 \times 10^{4}\end{array}$ & $\begin{array}{l}1.00 \\
0.56 \\
0.75 \\
0.38\end{array}$ & $\begin{array}{c}5.8 \times 10^{2} \\
12.2 \times 10^{-2} \\
3.95 \times 10^{0} \\
12.9 \times 10^{-3}\end{array}$ \\
\hline
\end{tabular}

$\bar{\alpha}_{f_{u}}$ is the $F(\alpha, n)$ yield correction factor (relative to ${ }^{234} \mathrm{U}$ ) which accounts for effect of different alpha particle energies.

calculate the neutron yields of the isotopes of normal uranium $\left(0.71 \%{ }^{235} U /{ }^{234} U=125\right)$ shown in Table 9.5. An approximate expression fos the neutron yield of UF6 is

$S($ neutrons $/ s)=M_{U}\left(0.0285+5.76 I_{234}\right)$

(9.1)

where

$M_{U}=$ mass of uranium in grams, and

$I_{234}={ }^{234} \mathrm{U}$ isotopic abuindance in percent.

Estimates of the neutron source strengths of $2268 \mathrm{~kg}$

TABLE 9.5

UF' NEUTRON YIELDS
OF NORMAL URANIUM

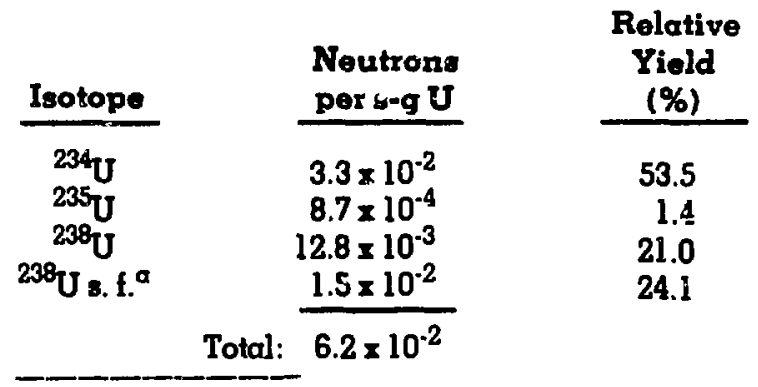

aspontaneous fission.
$(5000 \mathrm{lb})$ of normal $\mathrm{JF}_{6}$ and $3 \%$ enriched $\mathrm{UF}_{6}$ are 9.4 × $10^{4}$ and $2.6 \times 10^{5} \mathrm{n} / \mathrm{s}$, respectively.

Passive neutron counting of $\mathrm{UF}_{6}$ cylinders is one of the fastest, simplest, and least expensive methods for verification $( \pm 6 \%, 1 \sigma)$ of low enriched $\mathrm{UF}_{6}$. If either the spocific neutron yield of the UF 6 or the ${ }^{234} \mathrm{U}$ isotopic abundance were included in the cylinder specifications from the originating station. passive neutror counting would provide an accurate verification procedure.

\subsection{Sponianeous Fission Coincidence Coun- ting}

To avoid the ambiguities caused by variable $(\alpha, n)$ vields trom ussay samples, neutron coincidense counting $^{9.5,9.6}$ of spontaneous fission neutrons provides a useful assay technique if the isotopic composition is known. During a spontaneous tission reaction, two to three neutrong are emitted at essontially the same time. Neutron coincidence detectors are designed to separate the multiple neutron emission events from the $(\alpha, n)$ background neutrons.

Because the spontaneous lission rates are rather low (Table 7.4), high-efficiency detection systems are essential for rapid, quantitative assay. Thermalneutron detectors having ${ }^{3} \mathrm{He}$ or $\mathrm{BF}_{3}$ proportional counters and polyethylene moderators with efficiencies as large as $60 \%$ for total neutron detection have been developed for this purpose. These detectors completely surrourd the sample. The sensitivity of the units with high efficiency is $\sim 1 \mathrm{mg}{ }^{240} \mathrm{Pu}$ and 50 $g^{238} \mathrm{U}$, and the variable neutron die-away time (the average time for fission spectrum neutrons to thermalize) makes it possible to assay a wide range of 
plutonium loading ( $\sim 1$ to $1000 \mathrm{~g}$ plutonium). The long slowing-down times (10- to $150-\mu \mathrm{s})$ require correspondingly large coincidence gate times, and this zesults in sizable accidental neutron background rates-especially in samples with high-reutron emission rates.

In addition to the thermal-neutron detectors, organic scintillation detectors have been used for coincidence detection of spontaneous fiesion. $9.7,9.8$ Usually these scintillators detect both fast neutrons and gamma rays emitted during the fission process, therefore the effective multiplicity of detectable emissions is greatly increased because there are about three times more prompt gamma rays than prompt neutrons. There is no slowing down process required in the detector, therefore the coincidence gate times are $\sim 10^{3}$ times shorter than for thermal. neutron detectors. These short coincidence gate times $(\sim 50 \mathrm{ng})$ result in a small accidental background rate even for high count rate samples. However, the assay results are more dependent on sample geometry and matrix materials. Mony of the problem areas oncountered in using neutron coincidence counters are summarized elsewhere. ${ }^{9.9}$ An additional problem area, namely criticality, is discussed in Appendix C.

\section{REFERENCES}

9.1 J. E. Foley, Los Alamos Scientific Laboratory A1 Progress Report, LA-4805-MS (1970), p. 25. The tithe of this article is " $4 \pi$-Neutron Counter for 55 Gallon Barrels."
9.2. T. L. Atwell, D. B. Smith, and A. C. Wolker, Los Alamos Scientitic Laboratory A-1 Progress Report, LA-5431-PR (1973), p. 20. The title of this article is "Assay of Plutonium Metal Buttons with a Portable Neutron Counter."

9.3. R. B. Walton, T. D. Reilly, J. L. Parker, J. H. Menzel, E. D. Marshall, and L. W. Fields, "Measurements of $\mathrm{JF}_{5}$ Cylinders with Portable Instruments," Nucl. Tech. 21, No. 2, 133, (1974).

9.4. Thomas E. Sampson, "Neutron Yields from Uranium Isotopes in Uranium Hexafluoride," Nucl. Engr. Sci. 54, No. 4, 470 (1974).

9.5. G. R. Keepin, "LASL Safeguards Fiesearch and Development Program،" Proc AEC Symposium on Safeguards Research and Development, Los Alamos, NM (1959), WASH-1147, p. 114.

9.6. R. Shei, "Operating Charactaristics of Neutron Well Coincidence Counters," Brookhoven National Laboratory repost BNL 50332, TSO, BNL (1972).

9.7 T. Gozani and D. G. Costello, "Isotopic Source Assay System for Nuclear Materials," Trans. Amer. Nucl. Soc. 13, 2, 746 (1970).

3.8. R. B. Walton and A. C. Berick, Los Alamos Scientific Laboratori A-1 Progress Report, LA-1070MS (1969), p. 20. The title of this article is "Gamma Ray 'SLAB' Scinfillation Detector."

9.9. H. O. Menlove, "Do's and Dont's of Nondestructive Assay Measurements," LASL Internal Document. 


\section{APPENDIX $A$}

\section{GAMMA-RAY GIGNATURES}

This is a linting of the gamma raye from the fissionable nuclides. A.1 The brenching intensity is given in gammon/diaintegration and the orror is given in percent. These mearurements are being repeated and on updated listing should be available by 1975 .

\section{RHFHandes}

A.1. R. Gunnink and J. F. Tinnoy, "Analyeis of Fuel Rods by Gamma-Roy Spectroscopy," Lawrence Livermore Laboratory report UCRL.S1086 (1971). 


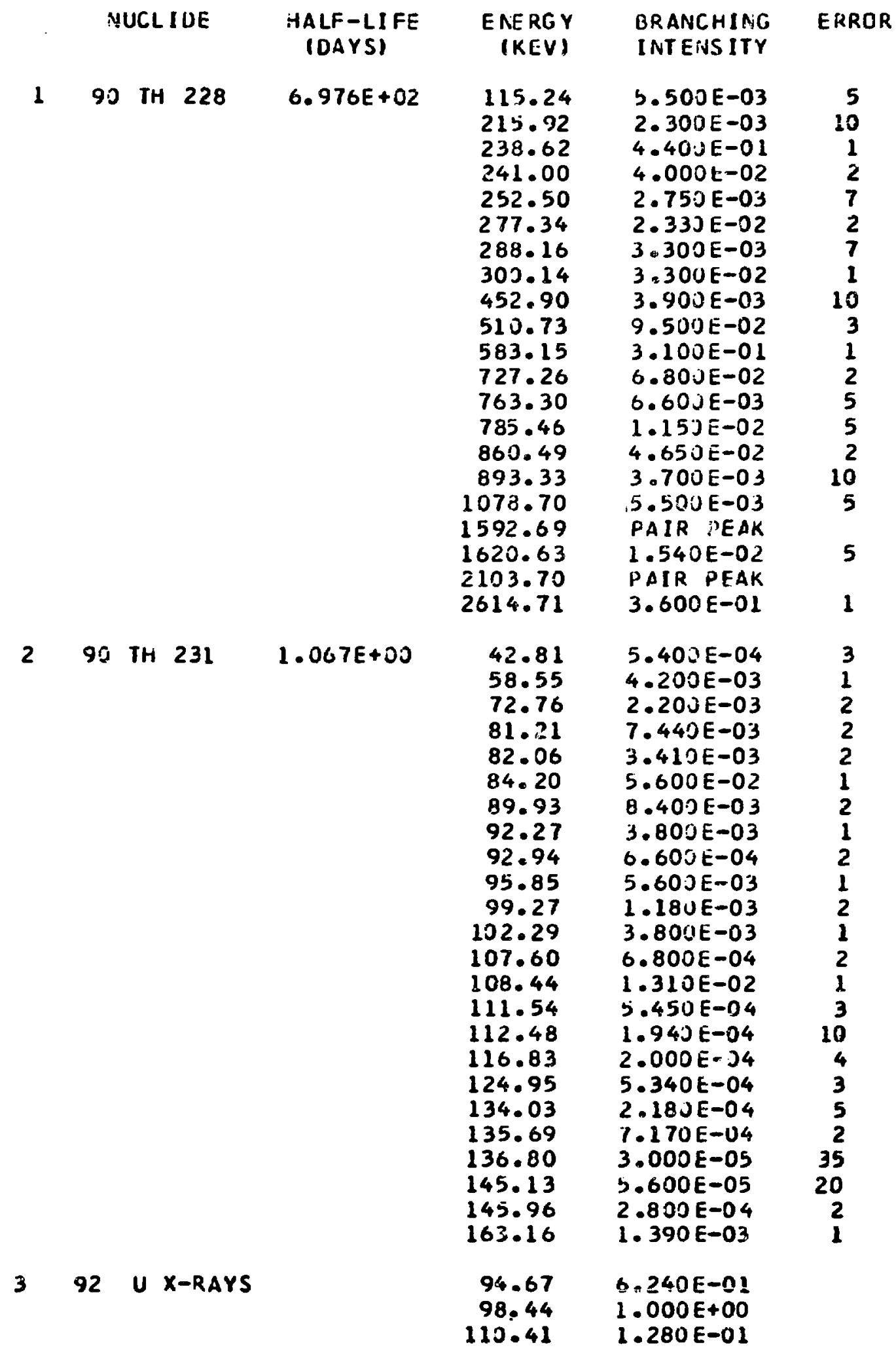


VUCLIDE

HALF-LIFE (DAYS)

$392 U$ X-RAYS

4
$92 \cup 235$

\begin{abstract}
$2.604 E+11$
\end{abstract}

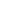

ENERGY

(KEV)

BRANCHING

ERROK

I NTENS ITY

$\begin{array}{ll}111.29 & 2.320 E-01 \\ 114.50 & 9.300 E-02 \\ 115.40 & 3.00 J E-02\end{array}$

$\begin{array}{rrr}89.96 & 2.450 E-02 & 3 \\ 93.35 & 4.000 E-02 & 2 \\ 104.82 & 4.560 E-03 & 2 \\ 105.61 & 9.100 E-03 & 2 \\ 140.75 & 2.08 J E-03 & 5 \\ 143.77 & 1.070 E-01 & 1 \\ 163.37 & 4.850 E-02 & 1 \\ 182.57 & 3.600 E-03 & 3 \\ 185.72 & 5.610 E-01 & 1 \\ 194.94 & 6.150 E-03 & 1 \\ 202.10 & 1.07 J E-02 & 1 \\ 205.33 & 4.870 E-02 & 1 \\ 215.35 & 2.750 E-04 & 3 \\ 233.54 & 2.750 E-04 & 2 \\ 240.93 & 6.40 J E-04 & 4 \\ 246.88 & 5.20 J E-04 & 4 \\ 275.34 & 2.690 E-04 & 3 \\ 291.63 & 2.000 E-04 & 10 \\ 345.89 & 3.00 J E-04 & 5 \\ 387.81 & 1.200 E-04 & 6\end{array}$
92
U 237
$6.75 O E+00$

$$
\begin{aligned}
& 25.75 \\
& 33.51 \\
& 51.09 \\
& 59.54 \\
& 64.02 \\
& 97.08
\end{aligned}
$$

101.07

113.2 .7

114.20

124.84

117.47

118.40

164.59

207.97

221.80

267.45

332.29

335.33

368.70

370.80

$2.390 \leq-02$

$1.240 E-03$

$5.150 E-03$

3. $62 J E-01$

1.370E-02

$1.475 E-01$

2. $355 E-01$

$2.88 .3 E-02$.

5.40JE-02

$3.700 E-03$

2. $260 E-02$

$7.800 E-03$

1. $830 \varepsilon-02$

2. OBOE-O1

2. 200 E-04

$7.200 E-03$

$1.140 E-02$

$1.100 E-03$

4. 300 E-04

1.05JE-03

$$
\begin{array}{r}
2 \\
6 \\
4 \\
1 \\
2 \\
1 \\
1 \\
1 \\
1 \\
40 \\
3 \\
10 \\
2 \\
2 \\
20 \\
2 \\
2 \\
5 \\
2 \\
10
\end{array}
$$

$6 \quad 92 \cup 238$

\section{$1.647 E+12$}
258.23
294.90
369.50
371.90

7.300E-04

3

4.800E-05

4.800E-05

$2.300 E-05$

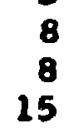




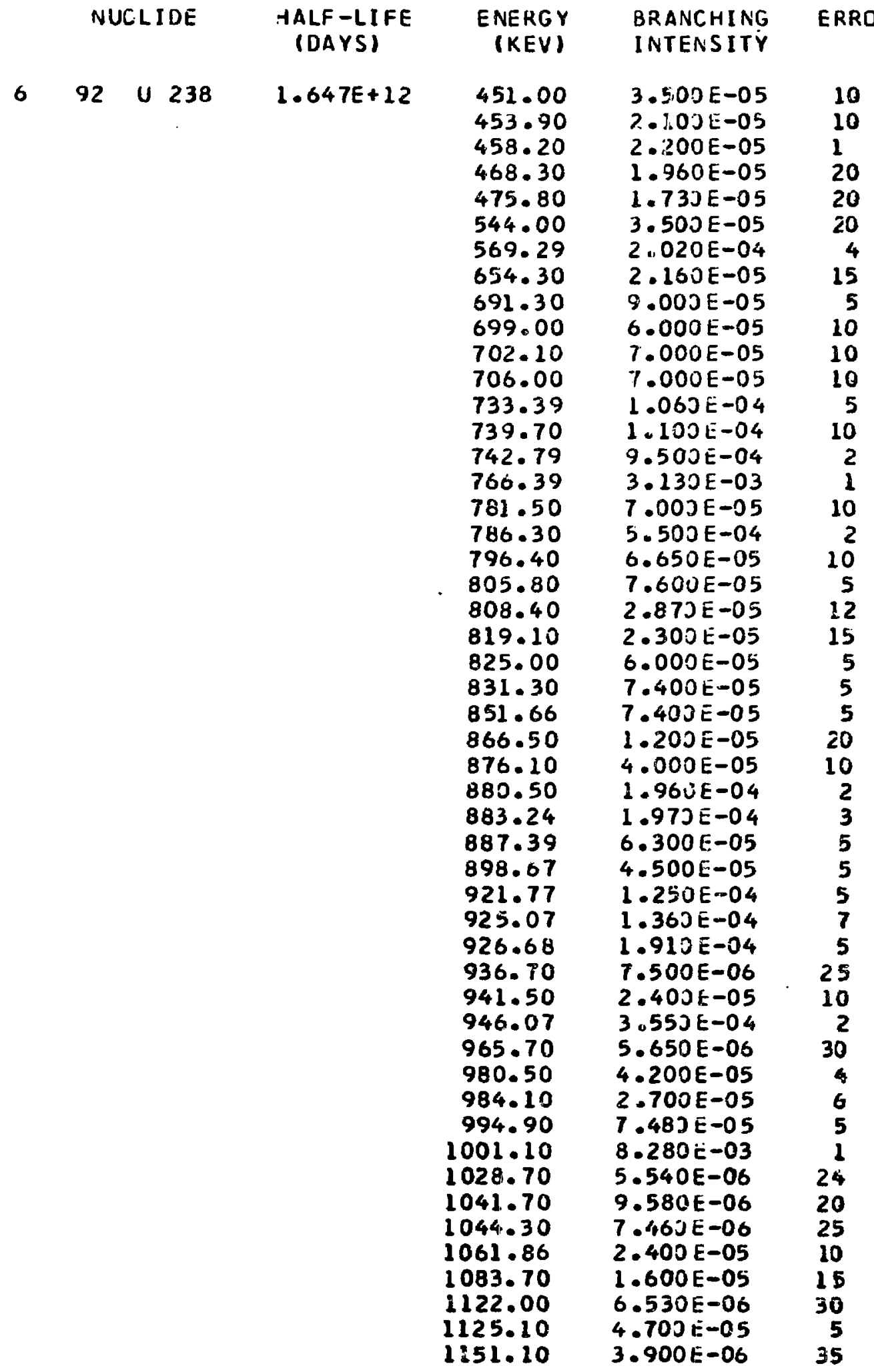


NUELIDE

6

$$
\begin{aligned}
& \text { PALF - LIFE } \\
& \text { (DAYS) }
\end{aligned}
$$

$92 \cup 238 \quad 1.647 E+12$

\section{ENERGY (KEV)}

BRANCHING

\begin{tabular}{|c|c|}
\hline $\begin{array}{l}1193.76 \\
1220.10 \\
1237.26 \\
1292.70 \\
1320.50 \\
1352.90 \\
1393.50 \\
1413.90 \\
1434.27 \\
1445.80 \\
1452.60 \\
1510.32 \\
1527.25 \\
1548.24 \\
1553.80 \\
1558.90 \\
1570.80 \\
1593.20 \\
1593.20 \\
1638.10 \\
1654.10 \\
1668.40 \\
1685.80 \\
1694.30 \\
1716.50 \\
1737.91 \\
1750.20 \\
1760.05 \\
1765.80 \\
1796.70 \\
1809.25 \\
1819.80 \\
1831.48 \\
1663.00 \\
1867.48 \\
1874.77 \\
1877.00 \\
1890.50 \\
1893.65 \\
1911.21 \\
1925.78 \\
1937.06 \\
1969.04 \\
2022.50 \\
2041.00 \\
2065.50 \\
2136.40\end{array}$ & $\begin{array}{l}1.340 E-05 \\
8.750 E-06 \\
4.900 E-05 \\
8.600 E-06 \\
2.90 J E-06 \\
2.24 J E-05 \\
3.700 E-05 \\
2.310 E-05 \\
9.000 E-05 \\
4.00 J E-06 \\
9.500 E-06 \\
1.300 E-04 \\
2.18 J E-05 \\
1.400 E-05 \\
8.200 E-05 \\
7.000 E-06 \\
1.05 J E-05 \\
2.650 E-05 \\
3.400 E-06 \\
3.800 E-06 \\
3.300 E-06 \\
1.200 E-05 \\
2.970 E-06 \\
1.280 E-05 \\
1.940 E-06 \\
2.120 E-04 \\
1.610 E-06 \\
1.440 E-05 \\
7.60 J E-25 \\
5.700 E-05 \\
3.700 E-05 \\
8.550 E-06 \\
1.750 E-04 \\
1.150 E-04 \\
9.150 E-05 \\
7.800 E-05 \\
1.930 E-05 \\
2.000 E-06 \\
2.130 E-05 \\
6.100 E-05 \\
5.703 E-06 \\
2.870 E-05 \\
4.000 E-06 \\
1.870 E-06 \\
8.970 E-07 \\
8.000 E-07 \\
8.250 E-07\end{array}$ \\
\hline $\begin{array}{r}99.54 \\
103.75\end{array}$ & $\begin{array}{l}6.310 E-01 \\
1.000 E+00\end{array}$ \\
\hline
\end{tabular}
INTENSITY
ERROR

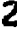

12

2

6

8

2

15

10

32

30

10

5

40

1

2

8

2

8

25

25 
NUCL I DE

7

94 PU X-RAYS

8

$94 \mathrm{PU} 238$
HALF-LIFE (DAYS)

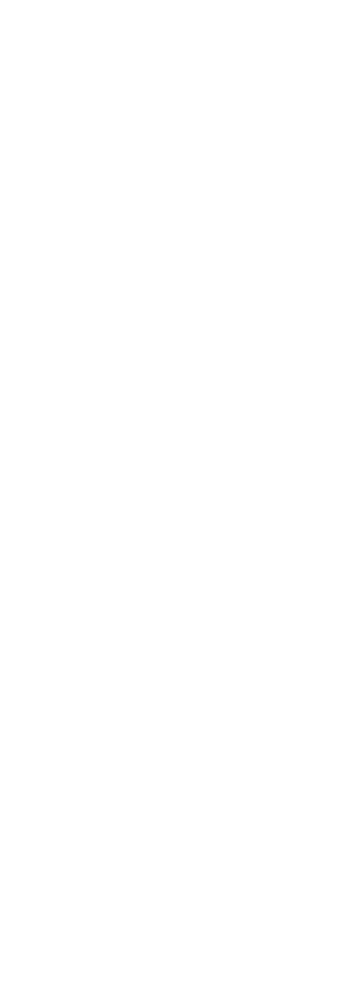

$3.200 E+04$

94 PU 239

$8.908 E+06$

ENE RG Y

(KEV)

BRANC+ING

INTENS:TY

$\begin{array}{ll}116.22 & 1.280 E-01 \\ 117.20 & 2.320 E-01 \\ 120.60 & 9.300 E-02 \\ 121.60 & 3.000 E-02\end{array}$

43.49

94.67

98.44

99.87

110.41

111.29

114.45

152.77

200.98

252.41

258.23

299.10

705.60

708.40

742.82

766.41

$786 \cdot 30$

805.42

808.23

851.73

883.21

904.34

926.73

942.02

946.12

1001.20

1041.90

1085.40

3.900 E-04

$9.000 E-07$

$1.480 E-06$

7.400 E- 05

1. $990 \mathrm{E}-07$

$3.070 E-07$

$1.160 E-07$

$1.010 \mathrm{E}-05$

4. 280 E-08

3. 53 J E -09

$6.770 \mathrm{E}-10$

4. $700 E-10$

$5.400 E-10$

5.380E-09

5. $550 E-08$

$2.400 E-07$

3.530E-08

1.390E-09

8. $580 \mathrm{E}-09$

1. $390 \mathrm{E}-08$

$6.530 E-09$

$6.210 \mathrm{E}-10$

$6.250 E-09$

$5.260 E-09$

$1.030 \mathrm{E}-09$

1. $300 E-08$

2. $290 E-09$

$8.850 E-10$

ERROR

30.09

32.24

36.39

37.28

38.69

40.57

46.23

47.56

51.63

54.05

56.83

65.69

67.66

68.73

74.96

77.60

$1.050 E-06$

1.37DE-05

4.230E-06

$1.030 E-06$

5.860 E-OS

$2.560 E-07$

5.110 E-06

2.870E-07

2.080 E-04

1.690 E-06

9.300 E-06

$3.350 E-07$

$1.420 E-06$

$5.990 E=00$

3.800 E-07

4.270 E-06 
NUCLIDE

9

94 PU 239
HALF-LIFE
(DAYS)

8. $908 E+06$
ENERGY (KEV)

$$
\begin{aligned}
& 78 \\
& 89 \\
& 94
\end{aligned}
$$

78.38

89.59

94.67

98.4 .4

$9 B .31$

103.03

110.41

111.29

114.32

115.35

116.24

119.72

123.67

124.52

125.17

129.28

141.64

144.19

146.05

158.30

$16 ! .45$

163.10

171.34

173.60

177.17

184.30

188.27

189.34

195.65

197.98

203.52

225.43

237.77

242.09

243.33

244.80

243.95

255.33

263.93

265.54

281.20

285.30

297.43

302.87

307.81

311.69

313.50

316.35

320.82

323.76
BRANCHING

INTENSITY

ERROR

$1.690 E-06 \quad 5$

1.340E-07

$3.670 E-05$

5.900E-05

1.300E-05

1.790 E-06

7.760 E-06

1. $470 E-05$

$5.590 E-06$

$6.760 E-06$

$5.960 E-06$

$3.030 E-07$

1. $680 E-07$

$6.230 E-07$

$5.790 E-07$

6. $200 E-05$

3.110E-07

2.840E-06

$1.130 E-06$

$8.000 E-08$

$1.300 \mathrm{E}-06$

$3.800 E-08$

$1.090 E-06$

4. $900 E-08$

$0.390 E-07$

$1.630 E-08$

$9.600 \mathrm{E}-08$

$7.700 E-07$

1. $070 E-06$

5.000E-08

$5.630 t-06$

$1.630 E-07$

$1.500 E=0$ ?

$8.320 E-08$

$2.320 E-07$

$5.230 E-08$

$7.50 J E-08$

$8.030 \mathrm{E}-07$

$2.550 E-07$

$2.853 E-08$

2.140E-08

1.500E-08

5. $000 E-07$

5. $700 E-08$

$6.200 E-08$

$2.740 E-07$

2.000 E-09

$1.410 E-07$

$5.690 E-07$

5.390E-O7 
NUCL IOE

9
HALF-LIFE
(DAYS)

94 HU $239 \quad 8.908 E+06$

\section{ENERGY}

(KEV)

332.80

336.06

341.48

344.96

354.10

361.90

367.02

368.53

375.02

380.16

382.70

392.37

393.04

399.44

410.77

413.69

422.57

426.67

430.00

445.78

451.45

457.57

461.29

463.80

474.40

481.55

487.00

493.10

538.90

550.60

557.70

586.40

598.10

607.30

612.90

617.40

618.90

624.80

633.19

637.97

640.15

646.02

649.50

652.19

654.36

658.99

664.67

374.20

686.16

690.85
BRANCHING ERROR

I NTENSITY

$5.050 E-06 \quad 1$

1. 130 E-06

$6.630 E-07$

$5.610 E-06$

8.000 E-09

1. $170 \mathrm{E}-07$

8.720E-07

8.96JE-07

1. $585 E-05$

3. 075 E-06

2.600 E-0

$1.160 E-00$

4. $440 E-06$

$6.13 J E-08$

8. 000 E-08

1. $506 E-05$

1. $190 \mathrm{E}-06$

2.290 E-07

4.910E-08

$9.100 E-08$

1. $920 E-06$

$1.540 E-08$

$2.020 E-08$

2. 570E-09

$9.420 E-10$

4. $770 E-08$

2.500E-09

$8.800 E-09$

3.000 E-09

$4.000 E-09$

$6.00 J E-10$

1. $420 E-09$

$1.900 E-08$

$1.450 \mathrm{E}-09$

8. $10 J E-09$

$2.04 J E-08$

$2.440 E=08$

4.000 E-D9

2. 320E-08

2. $500 E-08$

7.950 E-OB

1. $450 E-07$

8. 100 E-09

6. $400 E-08$

2. 150 E-08

9.500 E-OB

1.540E-08

5. 20DE-09

8. $900 E-09$

5.500 E-09
1

1

1

40

3

1

1

1

1

10

3

5

50

1

1

3

6

4

1

7

10

30

30

2

15

6

20

10

40

15

3

15

4

4

4

6

3

5

3

2

20

3

8

2

4

4

4 


\begin{tabular}{|c|c|c|c|c|c|c|c|}
\hline & \multicolumn{3}{|c|}{ NUCL IUE } & $\begin{array}{c}\text { HALF-LIFE } \\
\text { (DAYS) }\end{array}$ & $\begin{array}{c}\text { ENERGY } \\
\text { (KEV) }\end{array}$ & $\begin{array}{l}\text { BRANCHING } \\
\text { INTENSITY }\end{array}$ & ERI \\
\hline 9 & 94 & $4 \mathrm{PU}$ & 239 & $8.908 E+06$ & $\begin{array}{l}701.00 \\
703.79 \\
717.76 \\
727.81 \\
756.40 \\
769.38 \\
779.50 \\
787.21 \\
793.00 \\
796.50 \\
803.28 \\
808.14 \\
813.89 \\
821.02 \\
828.61 \\
832.06 \\
840.00 \\
843.75 \\
878.96 \\
891.00 \\
940.00 \\
956.40 \\
979.50 \\
986.70 \\
992.40 \\
1005.50 \\
1057.30\end{array}$ & $\begin{array}{l}5.400 E-09 \\
3.880 E-08 \\
2.670 E-08 \\
1.090 E-09 \\
3.370 E-08 \\
1.100 E-07 \\
1.330 E-09 \\
8.400 E-10 \\
2.500 E-10 \\
3.200 E-10 \\
4.450 E-10 \\
1.470 E-09 \\
6.200 E-10 \\
5.110 E-10 \\
1.380 E-09 \\
2.540 E-10 \\
2.800 E-10 \\
1.500 E-09 \\
3.210 E-10 \\
7.760 E-10 \\
4.200 E-10 \\
5.600 E-10 \\
2.260 E-10 \\
1.200 E-10 \\
2.260 E-10 \\
1.200 E-10 \\
4.500 E-10\end{array}$ & $\begin{array}{l}20 \\
30 \\
10 \\
15 \\
15 \\
15 \\
15 \\
20 \\
40 \\
20 \\
35 \\
15\end{array}$ \\
\hline 10 & 94 & $P U$ & 240 & $2.410 E+06$ & $\begin{array}{r}45.24 \\
94.57 \\
98.44 \\
104.23 \\
111.29 \\
114.50 \\
160.35 \\
642.30 \\
687.63\end{array}$ & $\begin{array}{l}4.500 E-04 \\
6.150 E-07 \\
1.00 J E-06 \\
7.000 E-05 \\
3.600 E-07 \\
1.230 E-07 \\
4.200 E-06 \\
1.450 E-07 \\
3.700 E-08\end{array}$ & $\begin{array}{r}1 \\
10 \\
5 \\
1 \\
10\end{array}$ \\
\hline 11. & 94 & $P U$ & 241 & $2.070 E+08^{* *}$ & $\begin{array}{r}44.19 \\
44.83 \\
56.30 \\
71.60 \\
76.96 \\
94.67 \\
98.47 \\
103.67 \\
110.42 \\
111.30 \\
111=96 \\
114.50\end{array}$ & $\begin{array}{l}1.820 E-03 \\
4.500 E-04 \\
1.500 E-03 \\
1.100 E-03 \\
9.800 E-03 \\
1.210 E-01 \\
1.910 E-01 \\
4.210 E-02 \\
2.390 E-02 \\
4.410 E-02 \\
1.720 E-03 \\
1.820 E-02\end{array}$ & $\begin{array}{r}3 \\
10 \\
5 \\
5 \\
1 \\
1 \\
1 \\
1 \\
1 \\
2\end{array}$ \\
\hline
\end{tabular}


NUCL IDE

$$
\begin{aligned}
& \text { TALF-LIFE } \\
& \text { (OAYS) }
\end{aligned}
$$

E AE RGY

BRANCHING IRTTENSITY

ERROR

1194 PU $241 \quad 2.070 E+03$

115.41
148.30
160.00

5. $400 E-03$

7. 74 IJ -02

2.620 E-03

2

1

1295 AM $241 \quad 1.582 E+05$

$\begin{array}{rrr}43.40 & 9.100 E-04 & 20 \\ 59.54 & 3.59 J E-01 & 1 \\ 69.90 & 2.010 E-05 & 6 \\ 97.10 & 1.130 E-05 & 7 \\ 99.00 & 2.020 E-04 & 2 \\ 101.09 & 1.880 E-05 & 5 \\ 103.00 & 1.950 E-04 & 2 \\ 113.27 & 2.400 E-06 & \\ 114.20 & 4.780 E-06 & \\ 118.00 & 2.210 E-06 & \\ 123.01 & 1.020 E-05 & 3 \\ 125.30 & 3.950 E-05 & 3 \\ 146.55 & 4.580 E-06 & 3 \\ 150.12 & 7.200 E-07 & 4 \\ 158.80 & 1.140 E-08 & 50 \\ 161.60 & 2.30 J E-08 & 20 \\ 164.60 & 6.200 E-07 & 4 \\ 165.95 & 2.430 E-07 & 5 \\ 169.57 & 1.680 E-06 & 3 \\ 175.16 & 1.840 E-07 & 6 \\ 191.90 & 2.200 E-07 & 4 \\ 194.70 & 1.70 J E-08 & 25 \\ 204.00 & 3.200 E-08 & 25 \\ 207.98 & 7.600 E-06 & 2 \\ 221.49 & 4.000 E-07 & 4 \\ 233.02 & 4.80 J E-08 & 10 \\ 234.40 & 6.000 E-09 & 40 \\ 246.72 & 2.000 E-08 & 25 \\ 260.98 & 8.600 E-09 & 30 \\ 264.92 & 8.270 E-03 & 5 \\ 267.50 & 2.450 E-07 & 3 \\ 275.72 & 6.220 E-08 & 6 \\ 291.15 & 3.150 E-08 & 15 \\ 292.72 & 1.310 E-07 & 5 \\ 300.09 & 3.740 E-08 & 8 \\ 304.16 & 8.000 E-09 & 35 \\ 311.94 & 3.900 E-07 & 3 \\ 322.48 & 1.440 E-06 & 2 \\ 332.33 & 1.450 E-06 & 2 \\ 335.38 & 4.700 E-06 & 2 \\ 337.69 & 3.050 E-08 & 15 \\ 343.40 & 2.500 E-08 & 25 \\ 350.40 & 1.400 E-08 & 30 \\ 358.20 & 1.510 E-08 & 20 \\ 366.00 & 2.080 E-06 & 2 \\ 370.91 & 4.910 E-07 & 2\end{array}$


NUCL IDE

$12954 M 241$

\author{
HALF-LIFE \\ (DAYS)
}

1. $582 E+05$

ENERjY

(KEV)

376.57

383.74

390.53

398.50

406.46

415.90

419.19

426.34

429.60

442.75

452.23

454.58

459.40

467.90

486.30

$514 \cdot 10$

521.90

574.00

586.52

590.30

597.39

618.96

626.90

632.80

641.37

652.88

662.37

675.70

679.99

688.70

693.41

696.34

709.30

721.92

729.46

737.22

755.87

759.37

766.87

770.93

780.42

811.80

851.90

862.90
BRANCHING I ATENSITY

ERRCR

1.330 E-06

$2.90 J E-07$

$6.100 t-08$

1. $500 E-08$

1. 200 E-08

1.61JE -08

2.760E-07

2. 400 E- 07

$5.000 E-09$

$3.40 J E-08$

1. $700 E-08$

9.300E-08

3. $200 E-08$

$2.50 J E-08$

1. 200 E -08

2. $700 \mathrm{E}-08$

7.000E-09

$1.450=-08$

$1.430 E-08$

$1.520 E-08$

6.500E-08

$5.55 U E-07$

3.60JE-09

1. $100 \mathrm{E}-08$

$6.450 E-08$

$3.54 J E-07$

$3.460 E-06$

$6.800 E-09$

$2.600 E-08$

$2.92 J E-0 T$

3.160E-05

4. $900 E-08$

$5.800 E-08$

$1.85 J E-06$

1. $300 E-08$

$7.500 E-08$

$7.100 \mathrm{E}-0 \mathrm{~B}$

l. 653 E-08

5.100 E-08

$6.470 E-08$

2.700E-09

5. $20 J$ E-09

2. BOOE-09

5. 100 E-09
1

1

5

30

35

25

3

4

50

20

25

6

15

25

40

30

50

20

20

20

5

2

50

25

4

2

1

30

10

2

5

4

4

1

15

3

3

8

4

4

50

2

45

30 


\begin{tabular}{|c|c|c|c|c|c|c|c|}
\hline \multirow[b]{2}{*}{1} & \multicolumn{3}{|c|}{ NUCLIDE } & \multirow{2}{*}{$\begin{array}{l}\text { ENERGY } \\
\text { (KEV) } \\
26.75\end{array}$} & \multirow{2}{*}{$\begin{array}{l}\text { BRANCHING } \\
\text { I RTENSITY } \\
2.390 E-02\end{array}$} & \multirow{2}{*}{$\begin{array}{c}\text { ASSCCIATIVE } \\
59.54\end{array}$} & \multirow{2}{*}{$\begin{array}{r}\text { GAMY I RA } \\
207.97\end{array}$} \\
\hline & 92 & $u$ & 237 & & & & \\
\hline 2 & 94 & PU & 239 & 30.09 & $1.050 E-0.6$ & 51.63 & 98.44 \\
\hline 3 & 94 & PU & 239 & 32.24 & $1.370 E-05$ & 51.63 & 98.44 \\
\hline 4 & 92 & $\mathbf{U}$ & 237 & 33.51 & $1.2 \div 0 E-03$ & 59.54 & 207.97 \\
\hline 5 & 94 & PU & 239 & 36.39 & $4.230 E-06$ & 51.63 & 98.44 \\
\hline 6 & 94 & $P U$ & 239 & 37.28 & $1.030 E-06$ & 51.63 & 98.44 \\
\hline 7 & 94 & PU & 239 & 38.59 & $5.860 E-05$ & 51.63 & 98.44 \\
\hline 8 & 94 & PU & 239 & 40.57 & $2.560 E-07$ & 51.63 & 98.44 \\
\hline 9 & 90 & IH & 231 & 42.81 & $5.400 E-04$ & 84.20 & 185.72 \\
\hline 10 & 95 & $A M$ & 241 & 43.40 & $9.100 E-04$ & 59.54 & 0. \\
\hline 11 & 94 & PU & 238 & 43.49 & $3.900 E-04$ & 99.87 & 152.77 \\
\hline 12 & 94 & PU & 241 & 44.19 & $1.820 E-03$ & 59.54 & 148.60 \\
\hline 13 & 94 & PU & 241 & 44.83 & $4.500 E-04$ & 59.54 & 248.60 \\
\hline 14 & 94 & PU & 240 & 45.24 & $4.500 E-04$ & 134.23 & $160 \cdot 35$ \\
\hline 15 & 94 & PU & 239 & 46.23 & $5.110 E-06$ & 51.63 & 98.44 \\
\hline 16 & 94 & $\mathbf{P U}$ & 239 & 47.56 & $2.870 E-07$ & 51.63 & 98.44 \\
\hline 17 & 92 & $\mathbf{U}$ & 237 & 51.09 & $5.1508-03$ & 59.54 & 207.97 \\
\hline 18 & 94 & PU & 239 & 51.63 & $2.080 E-04$ & 94.67 & 98.44 \\
\hline 19 & 94 & $P U$ & 239 & 54.05 & $1.690 \varepsilon-06$ & 51.63 & 98.44 \\
\hline 20 & 94 & $P U$ & 241 & 56.30 & $1.500 E-03$ & 59.54 & 148.60 \\
\hline $2 i$ & 94 & $P U$ & 239 & 56.83 & $9.300 E-06$ & 51.63 & 98.44 \\
\hline 22 & 90 & TH & 231 & 58.55 & $4 \cdot 200 E-03$ & 84.20 & 285.72 \\
\hline 23 & 95 & AM & 241 & 59.54 & $3.590 E-01$ & 99.00 & 303.00 \\
\hline 24 & 92 & $\mathbf{u}$ & 237 & 59.54 & $3.620 E-01$ & 104.59 & 207.97 \\
\hline 25 & 92 & $\mathbf{u}$ & 237 & 64.82 & $1.370 E-02$ & 164,59 & 207.97 \\
\hline 26 & 94 & PU & 239 & 65.69 & $\cdot 3.350 E=07$ & $51 \cdot 63$ & 98.44 \\
\hline 27 & 94 & PU: & 239 & 67.60 & $1.420 E-06$ & 51.63 & 98.44 \\
\hline
\end{tabular}




\begin{tabular}{|c|c|c|c|c|c|c|c|}
\hline & & UCLI & I DE & $\begin{array}{l}\text { ENERGY } \\
\text { (KEV) }\end{array}$ & $\begin{array}{l}\text { BRANCH ING } \\
\text { INTENSI TY }\end{array}$ & ASSCCIATIVE & GAMMA RAY \\
\hline 28 & 94 & PU & 239 & 68.73 & $5.990 E-06$ & 51.63 & 98.44 \\
\hline 29 & 95 & $A M$ & 241 & 69.90 & 2. $010 E-05$ & 59.54 & 99.00 \\
\hline 30 & 94 & PU & 241 & 71.60 & $1.100 E-03$ & 59.54 & 148.60 \\
\hline 31 & 90 & IH & 231 & 72.76 & $2.200 E-03$ & 64.20 & 185.72 \\
\hline 32 & 94 & PU & 239 & 74.96 & $3.800 E-07$ & 94.67 & 129.28 \\
\hline 33 & 94 & PU & 241 & 76.96 & $9.800 E-03$ & 59.54 & 248.60 \\
\hline 34 & 94 & pu & 239 & 77.60 & $4.270 E-06$ & 94.67 & 98.44 \\
\hline 35 & 94 & PU & 239 & 78.38 & $1.690 \mathrm{E}-06$ & 94.67 & 98.44 \\
\hline 36 & 90 & TH & 231 & 81.21 & $7.440 E-03^{\circ}$ & $84 \cdot 20$ & 285.72 \\
\hline 37 & 90 & TH & 231 & 82.06 & $3.410 E-03$ & 84.20 & 185.72 \\
\hline 38 & 90 & TH & 231 & 84.20 & $5.600 E-02$ & 89.93 & 285.72 \\
\hline 39 & 94 & pu & 239 & 89.59 & $1.340 E-07$ & 94.67 & 98.44 \\
\hline 40 & 90 & TH & 231 & 89.93 & $8.400 E-03$ & 84.20 & 185.72 \\
\hline 41 & 92 & $u$ & 235 & 89.96 & $2.450 E-02$ & 84.20 & 285.72 \\
\hline 42 & 90 & TH & 231 & 92.27 & $3.800 E-03$ & 84.20 & 285.72 \\
\hline 43 & 90 & $\boldsymbol{T}_{\mathbf{H}}$ & 231 & 92.94 & $6.600 E-04$ & $84 \cdot 20$ & 185.72 \\
\hline 44 & 92 & $\mathbf{u}$ & 235 & 93.35 & $4.000 E-02$ & 84.20 & 185.72 \\
\hline 45 & 94 & $\mathbf{P u}$ & 241 & 94.67 & $1.210 E-01$ & 59.54 & 248.60 \\
\hline 46 & 94 & PU & 240 & 94.67 & $6.150 E-07$ & 45.24 & 304.23 \\
\hline 47 & 94 & pu & 239 & 94.67 & $3.670 E-05$ & 98.44 & 129.28 \\
\hline 48 & 94 & PU & 238 & 94.67 & $9.000 E-07$ & 43.49 & 99.87 \\
\hline 49 & 92 & $\mathbf{u}$ & i-RAY & 94.67 & $6.240 E-01$ & 98.44 & $111 \cdot 29$ \\
\hline 50 & 90 & TH & 231 & 95.85 & $5.500 E-03$ & 84.20 & 285.12 \\
\hline 51 & 92 & $u$ & 237 & 97.08 & $1.475 E-01$ & 164.59 & 207.97 \\
\hline 52 & 95 & $A M$ & 241 & 97.10 & $1.130 E-05$ & 59.54 & 99.00 \\
\hline 53 & 94 & PU & 241 & 98.94 & $1.91 \mathrm{JE}-01$ & 59.54 & 148.60 \\
\hline 54 & 94 & Pu: & 240 & 98.44 & $1.000 E-06$ & 45.24 & 204.23 \\
\hline
\end{tabular}




\begin{tabular}{|c|c|c|c|c|c|c|c|}
\hline & & UCLI & $1 \mathrm{DE}$ & $\begin{array}{l}\text { ENERGY } \\
\text { (KEV) }\end{array}$ & $\begin{array}{l}\text { BKANCHING } \\
\text { INTENS ITY }\end{array}$ & ASSOCIATIVE G & GAMHA RA \\
\hline 55 & 94 & Py & 239 & 90.44 & $5.900 E-05$ & 34.67 & 129.28 \\
\hline 56 & 94 & PU & 238 & 98.44 & $1.480 E-06$ & 43.49 & 99.87 \\
\hline 57 & 92 & $u$ & $X$ XAY & 98.44 & $1.000 \varepsilon+00$ & 94.67 & 111.29 \\
\hline 58 & 94 & PU & 239 & 98.31 & $1.300 E-05$ & 94.67 & 129.28 \\
\hline 59 & 95 & $A M$ & 241 & 99.00 & $2.020 E-04$ & 59.54 & 103.00 \\
\hline 60 & 90 & TH & 231 & 99.27 & $1.180 E-03$ & $84 \cdot 20$ & 185.72 \\
\hline 61 & 94 & PU & $X-R A Y$ & 99.54 & $6.310 E-01$ & 103.75 & 217.20 \\
\hline 62 & 94 & PU & 238 & 99.87 & $7.400 E-05$ & 43.49 & 152.77 \\
\hline 63 & 92 & $u$ & 237 & 101.07 & $2.355 E-01$ & 164.59 & 207.97 \\
\hline 64 & 95 & $A M$ & 241 & 101.09 & $1.880 E-05$ & 59.54 & 103.00 \\
\hline 65 & 90 & TH & 231 & 102.29 & $3.800 E-03$ & 84.20 & 185.72 \\
\hline 66 & 95 & $A M$ & 241 & 103.00 & $1.950 E-04$ & 59.54 & 99.00 \\
\hline 67 & 94 & Pu & 239 & 103.03 & $1.790 E-06$ & 94.67 & 129.28 \\
\hline 68 & 94 & PU & 241 & 103.67 & $4.210 E-02$ & 59.54 & 148.60 \\
\hline 69 & 94 & PU & $X-R A Y$ & 103.75 & $1.000 E+00$ & 99.54 & 117.20 \\
\hline 70 & 94 & PU & 240 & $10 \therefore 23$ & $7.000 E-05$ & 45.24 & 160.35 \\
\hline 71 & 92 & $u$ & 235 & 104. 62 & $4.560 E-03$ & 84.20 & 185.72 \\
\hline 72 & 92 & $v$ & 235 & 105.61 & $9.100 E-03$ & 84.20 & 185.72 \\
\hline 73 & 90 & TH & 231 & 107.60 & 6. BOOE -O4 & 84.20 & 185.72 \\
\hline 74 & 90 & IH & 231 & 108.44 & $1.310 E-02$ & 84.20 & 185.72 \\
\hline 75 & 94 & PU & 239 & 110.41 & $7.760 E-06$ & 98.44 & $129 \cdot 28$ \\
\hline 76 & 94 & PU & 238 & 110.42 & $1.590 E-07$ & $99.8 ?$ & 152.77 \\
\hline 77 & 92 & u & $X$ XRAY & 110.41 & $1.280 E-01$ & 94.67 & 98.44 \\
\hline 78 & 941 & PU & 241 & 110.42 & $2.390 E-02$ & 59.54 & 148.60 \\
\hline 79 & 94 & PU & 240 & 111.29 & $3.600 E-07$ & 104.23 & $160 \cdot 35$ \\
\hline 80 & 94 & PU & 239 & 111.29 & $1.470 E-05$ & 98.44 & 829.28 \\
\hline 82 & 94 & PU & 238 & 111.29 & 3.07OE-OT & 99.87 & 152.77 \\
\hline
\end{tabular}




\begin{tabular}{|c|c|c|c|c|c|c|c|}
\hline 82 & 92 & $u$ & $X$ XAY & 111.29 & $2.320 E-01$ & 94.67 & 98.44 \\
\hline 83 & 94 & PU & 241 & 111.30 & $4.410 E-02$ & 59.54 & 148.60 \\
\hline 04 & 90 & TH & 231 & 111.54 & $5.450 E-04$ & 84.20 & 185.72 \\
\hline 85 & 94 & PU & 241 & 111.96 & $1.720 t-03$ & 59.54 & 148.60 \\
\hline 86 & 90 & TH & 231 & 112.48 & $1.940 E-04$ & 84.20 & 185.72 \\
\hline 87 & 95 & $A M$ & 241 & 113.27 & $2.400 E-06$ & 59.54 & 163.00 \\
\hline 88 & 92 & $u$ & 237 & 113.27 & $2.880 E-02$ & 164.59 & 207.97 \\
\hline 89 & 95 & $A M$ & 241 & 114.20 & $4.780 E-06$ & 59.54 & 103.00 \\
\hline 90 & 92 & $\mathbf{U}$ & 237 & 114.20 & $5.400 \mathrm{E}-02$ & 164.59 & 207.97 \\
\hline 91 & 94 & PU & 239 & 114.32 & $5.590 E-06$ & 98.44 & 129.28 \\
\hline 92 & 94 & PU & 238 & 114.45 & $1.160 E-07$ & 99.87 & 152.77 \\
\hline 93 & 94 & PU & 241 & 114.50 & $1.620 E-02$ & 59.54 & 148.60 \\
\hline 94 & 94 & $P U$ & 240 & 114.50 & $1.230 E-07$ & 104.23 & $260 \cdot 35$ \\
\hline 95 & 92. & $\mathbf{u}$ & $X-R A Y$ & 114.50 & $9.300 E-02$ & 94.67 & 98.44 \\
\hline 96 & 92 & $u$ & 237 & 114.84 & $3.700 E-03$ & 154.59 & 207.97 \\
\hline 97 & 90 & IH & 228 & 115.24 & $5.500 t-03$ & 238.62 & $583 \cdot 15$ \\
\hline 98 & 94 & PU & 239 & 115.35 & $6.760 E-06$ & 98.44 & 129.28 \\
\hline 99 & 92 & $\mathbf{U}$ & $X$-RAY & 115.40 & $3.000 E-02$ & 94.67 & 98.44 \\
\hline 100 & 94 & PU & 241 & 115.41 & $5.400 E-03$ & 59.54 & 148.60 \\
\hline 101 & 94 & PU & $X-R A Y$ & 116.22 & $1.280 E-01$ & 99.54 & 203.75 \\
\hline 102 & 94 & PU & 239 & 116.24 & $5.960 E-06$ & 98.44 & 229.28 \\
\hline 103 & 90 & TH & 231 & 116.83 & $2.000 E-04$ & $84 \cdot 20$ & 185.72 \\
\hline 104 & 94 & $P U$ & $X-R A Y$ & $117 \cdot 20$ & $2.320 E-01$ & 99.54 & 103.75 \\
\hline 105 & 92 & $\mathbf{u}$ & 237 & 1.17 .47 & $3.260 E-02$ & 164.59 & 207.97 \\
\hline 106 & 95 & $\mathbf{A M}$ & 241 & $118 \cdot 00$ & $2.210 E-06$ & 59.54 & 103.00 \\
\hline 107 & 92 & $\mathbf{U}$ & 237 & 118.40 & $7.800 E-03$ & $164 \cdot 59$ & 207.97 \\
\hline 108 & 94 & PU & 239 & 119.72 & $3.030 E-07$ & 98.44 & 129.28 \\
\hline
\end{tabular}




\section{NUCLI DE}

ENERGY

BRANCHING INTENSITY

109

94 PU X-RAY 120.60

11094 PU X-RAY 121.60

$9.300 E-02$

3. $000 E-02$

11195 AM 241

123.01

$1.020 E-05$

$112 \quad 94$ PU 239

123.67

$1.680 E-07$

11394 PU 239

$$
124.52
$$

$6.230 E-07$

11490 TH 231

124.95

5.340E-04

$115 \quad 94$ PU 239

125.17

5. $790 E-07$

11695 AM 241

125.30

3. $950 E-05$

11794 PU 239

129.28

$6.200 E-05$

$11890 \mathrm{TH} \quad 231$

134.03

2. $180 E-04$

$11990 \mathrm{IH} 231$

135.69

$7.170 E-04$

12090 TH 231

136.80

3. $000 E-05$

$12192 \cup 235$

140. 75

2. $080 E-03$

12294 PU $239 \quad 1.41 .64$

3. $110 E-07$

$\begin{array}{llll}123 & 92 & 0 & 235\end{array}$

143.77

1. 070E-01

12494 PU 239

144.19

2. $340 E-06$

$12590 \mathrm{TH} 231$

145.13

5.600E-05

$126.90 \mathrm{rH} 231$

145.96

2. $800 E-04$

12794 PU 239

146.05

1. $130 E-06$

12895 AM 241

146.55

4.580E-06

$229 \quad 94$ PU 241

148.60

7. 740 E - 02

13095 AM 241

150.12

7.2 OOE-OT

13194 PU 238

152.77

$1.010 E-05$

13294 PU 239

158.30

B. $000 E-08$

13395 AM 241

158.80

$1.140 E-08$

13494 PU 241

160.00

2.620E-03

13594 PU 240

4 200E-06
ASSOCIATIVE GAMMA RAYS

\begin{tabular}{rr}
99.54 & 103.75 \\
99.54 & 103.75 \\
59.54 & 103.00 \\
98.44 & 129.28 \\
98.44 & 1.29 .28 \\
84.20 & 185.72 \\
98.44 & 129.28 \\
59.54 & 103.00 \\
98.44 & 375.02 \\
84.20 & 185.72 \\
84.20 & 185.72 \\
84.20 & 185.72 \\
\hline 185.72 & 163.37 \\
129.28 & 375.122 \\
185.72 & 163.37 \\
129.28 & 375.02 \\
84.20 & 185.72 \\
84.20 & 185.72 \\
129.28 & 375.02 \\
59.54 & 103.00 \\
\hline 164.59 & 207.97 \\
59.54 & 103.00 \\
99.87 & 766.41 \\
129.28 & 375.02 \\
59.54 & 103.00 \\
164.59 & 207.97 \\
45.24 & 104.23
\end{tabular}




\begin{tabular}{|c|c|c|c|c|c|c|c|}
\hline & & UCLI & I DE & $\begin{array}{l}\text { ENERGY } \\
\text { (KEV) }\end{array}$ & $\begin{array}{l}\text { BRANCHING } \\
\text { INTENSITY }\end{array}$ & ASSOCIATIVE & GAMNA RAY \\
\hline 136 & 94 & PU & 239 & 161.45 & $1.300 E-06$ & 129.28 & 375.02 \\
\hline 137 & 95 & AMH & 241 & 161.60 & $2.300 E-0 B$ & 59.54 & 103.00 \\
\hline 138 & 90 & $r_{H}$ & 231 & 163.16 & $1.390 E-03$ & 84.20 & 185.72 \\
\hline 139 & 92 & $\mathrm{u}$ & 235 & 163.37 & $4.850 E-02$ & 185.72 & 143.77 \\
\hline 140 & 92 & $\mathrm{U}$ & 237 & 164.59 & $1.830 t-02$ & 148.60 & 207.97 \\
\hline 141 & 95 & $A M$ & 241 & 164.60 & $6.200 E-07$ & 59.54 & 103.00 \\
\hline 142 & 95 & $A M$ & 241 & 165.95 & $2.430 E-07$ & 59.54 & 103.00 \\
\hline 243 & 94 & $P U$ & 239 & 168.10 & $3.800 E-08$ & 129.28 & 375.02 \\
\hline 144 & 95 & $A M$ & 241 & 169.57 & $1.680 E-06$ & 59.54 & 103.00 \\
\hline 145 & 94 & $P U$ & 239 & 171.34 & $1.090 E-06$ & 129.28 & 375.02 \\
\hline 146 & 94 & PU & 239 & 173.60 & $4.900 E-08$ & 129.28 & 375.02 \\
\hline 147 & 95 & $A M$ & 241 & 175.16 & $1.840 E-07$ & 59.54 & 103.00 \\
\hline 148 & 94 & PU & 239 & 179.17 & $6.390 E-07$ & 129.28 & 375.02 \\
\hline 149 & 92 & $u$ & 235 & 182.57 & $3.600 E-03$ & 185.72 & 163.39 \\
\hline 150 & 94 & PU & 239 & 184.30 & $1.630 E-08$ & 129.28 & 375.02 \\
\hline 151 & 92 & $\mathbf{u}$ & 235 & 185.72 & $5.610 E-01$ & 143.77 & 163.37 \\
\hline 152 & 94 & PU & 239 & 188.27 & $9.600 E-0 B$ & 129.28 & 375.02 \\
\hline 153 & 94 & pu & 239 & 189.34 & $7.760 E-07$ & 129.28 & 375.02 \\
\hline 154 & 95 & AM & 241 & 191.90 & $2.200 E-07$ & 59.54 & 103.00 \\
\hline 155 & 95 & AM & 241 & 194.70 & $1.700 E-08$ & 59.54 & 103.00 \\
\hline 256 & 92 & $u$ & 235 & 194.94 & $6.150 E-03$ & 185.72 & 163.37 \\
\hline 157 & 94 & PU: & 239 & 195.65 & $1.070 E-06$ & 129.28 & 375.02 \\
\hline 158 & 94 & PU: & 239 & 197.98 & $5.000 E-08$ & 129.28 & 375.02 \\
\hline 159 & 94 & PU & 238 & 200.98 & $4.280 E-08$ & 152.77 & 766.41 \\
\hline 160 & 92 & $\mathbf{u}$ & 235 & 202.10 & $1.070 E-02$ & 185.72 & 163.37 \\
\hline 161 & 94 & PU & 239 & 203.52 & $5.630 E-06$ & 129.28 & 375.02 \\
\hline 162 & 95 & $A M$ & 241 & 204.00 & $3.200 E-08$ & 59.54 & 103.00 \\
\hline
\end{tabular}




\begin{tabular}{|c|c|c|c|c|c|c|c|}
\hline & & $J C L I D$ & & $\begin{array}{l}\text { EVERGY } \\
\text { (KEY) }\end{array}$ & $\begin{array}{l}\text { BRA ACHING } \\
\text { I ATENSITY }\end{array}$ & ASSOCIATIVE & GANMA RA \\
\hline 163 & 92 & $u$ & 235 & 205.33 & $4.870 E-02$ & 185.72 & 163.37 \\
\hline 164 & 92 & u & 237 & 207.97 & $2.080 E-01$ & 264.59 & 148.60 \\
\hline 165 & 95 & $A M$ & 241 & 207.98 & $7.600 \mathrm{E}-06$ & 59.54 & 103.00 \\
\hline 166 & 92 & $u$ & 235 & 215.35 & $2.750 E-04$ & 185.72 & 163.37 \\
\hline 167 & 90 & TH & 228 & 215.92 & $2.300 E-03$ & 238.62 & 583.15 \\
\hline 168 & 95 & $A M$ & 241 & 221.49 & $4.000 E-07$ & 59.54 & 203.00 \\
\hline 169 & 92 & $u$ & 237 & $221 \cdot 80$ & $2.200 E-04$ & 164.59 & 207.97 \\
\hline 170 & 94 & PU & 239 & 225.43 & $1.630 \mathrm{E}-07$ & 129.28 & 375.02 \\
\hline 171 & 95 & $A M$ & 241 & 233.02 & $4.800 E-08$ & 59.54 & 103.00 \\
\hline 172 & 92 & $\mathbf{u}$ & 235 & 233.54 & $2.750 E-04$ & 185.72 & 163.37 \\
\hline 173 & 95 & $A M$ & 241 & 234.40 & 6. $C 00 E-09$ & 59.54 & 103.00 \\
\hline 174 & 94 & PU & $<39$ & 237.77 & $1.500 E-07$ & 129.28 & 375.02 \\
\hline 175 & 90 & $T H:$ & 228 & 238.62 & 4. $400 E-01$ & 2614.71 & 523.15 \\
\hline 176 & 92 & $\mathbf{u}$ & 235 & 240.93 & $6.400 E-04$ & 185.72 & 163.37 \\
\hline 177 & 90 & $T+i$ & 228 & 241.00 & $4.000 E-02$ & 2614.71 & 583.15 \\
\hline 178 & 94 & PU: & 239 & 242.09 & $8.320 E-08$ & 129.28 & 375.02 \\
\hline 179 & 94 & PU & 239 & 243.33 & 2. $320 E-07$ & 129.28 & 375.02 \\
\hline 180 & 94 & PU & 239 & 244.80 & $5.230 E-08$ & 129.28 & 375.02 \\
\hline 181 & 95 & $A M=$ & 241 & 246.72 & $2.000 E-08$ & 59.54 & 203.00 \\
\hline 182 & 92 & U & 235 & 246.88 & $5.200 E-04$ & 185.72 & 163.37 \\
\hline 183 & 94 & pu & 239 & 248.95 & $T=500 E-08$ & 129.28 & 375.02 \\
\hline 184 & 94 & PU: & 238 & 252.41 & $3.530 E-09$ & 152.77 & 766.41 \\
\hline 185 & 90 & TH 2 & 228 & 252.50 & $2.750 E-03$ & 238.62 & 583.15 \\
\hline 186 & 94 & PU 2 & 239 & 255.33 & $8.030 t-07$ & 129.28 & 375.02 \\
\hline 187 & 94 & PU 2 & 238 & 258.23 & $6.770 E-10$ & 152.77 & 766.41 \\
\hline 188 & 92 & U2 & 238 & 258.23 & $7.300 E-0.4$ & 766.39 & $1001 \cdot 10$ \\
\hline 189 & 95 & $A M 2$ & 241 & 260.98 & $8.600 E-09$ & 59.54 & 103.00 \\
\hline
\end{tabular}




\begin{tabular}{|c|c|c|c|c|c|c|c|}
\hline & & IUCLI & I DE & $\begin{array}{l}\text { ENERGY } \\
\text { (KEV) }\end{array}$ & $\begin{array}{l}\text { BRANCHING } \\
\text { INTENSITY }\end{array}$ & ASSCC IAT IVE & GAMMA RAY \\
\hline 290 & 94 & $\boldsymbol{P U}$ & 239 & 263.93 & $2.550 E-07$ & $129 \cdot 28$ & 375.02 \\
\hline 191 & 95 & AM & 241 & 264.92 & $6.270 E-08$ & 59.54 & 103.00 \\
\hline 192 & 94 & $P U$ & 239 & 265.54 & $2.850 E-08$ & 129.28 & 375.02 \\
\hline 193 & 92 & $u$ & 237 & 267.45 & $7.200 E-03$ & 164.59 & 207.97 \\
\hline 194 & 95 & $A M$ & 241 & 267.50 & $2.450 E-07$ & 59.54 & 103.00 \\
\hline 195 & 92 & $\mathbf{U}$ & 235 & 275.34 & $2.690 E-04$ & 185.72 & 163.37 \\
\hline 196 & 95 & $A M$ & 241 & 275.72 & $6.220 E-08$ & 59.54 & 103.00 \\
\hline 197 & 90 & $T H$ & 228 & 277.34 & $2.330 E-02$ & 238.62 & 583.15 \\
\hline 198 & 94 & PU & 239 & $281 \cdot 20$ & $2.14 O E-0 B$ & 129.28 & 375.02 \\
\hline 199 & 94 & PU & 239 & 285.30 & $1.500 E-08$ & 129.28 & 375.02 \\
\hline 200 & 90 & TH & 228 & 288.16 & $3.300 E-03$ & 238.62 & 583.15 \\
\hline 201 & 95 & $A M$ & 241 & $291 \cdot 15$ & $3.150 E-O E$ & 59.54 & 103.00 \\
\hline 202 & 92 & $u$ & 235 & 291.63 & $2.000 E-04$ & 185.72 & 163.37 \\
\hline 203 & 95 & $A M$ & 241 & 292.72 & $1.310 E-07$ & 55.54 & 203.00 \\
\hline 204 & 92 & $u$ & 238 & 294.90 & $4.800 E-05$ & 766.39 & $1001 \cdot 10$ \\
\hline 205 & 94 & PU & 239 & 297.43 & 5. O00E-07 & 129.28 & 375.02 \\
\hline $20 \epsilon_{1}$ & 94 & PU & 238 & 299.10 & $4.700 E-10$ & 152.77 & 766.41 \\
\hline 207 & 95 & $4 M$ & 241 & 300.09 & $5.740 E-08$ & 59.54 & 103.00 \\
\hline 208 & 90 & $T H$ & 228 & 300.14 & $3.300 E-02$ & 238.62 & 583.15 \\
\hline 209 & 94 & PU & 239 & 302.87 & $5.700 E-08$ & 129.28 & 375.02 \\
\hline 210 & 95 & AM & 241 & $304 \cdot 16$ & $8.000 E-09$ & 55.54 & 103.00 \\
\hline 211 & 94 & PU & 239 & 307.81 & $6.200 E-08$ & 129.28 & 375.02 \\
\hline 212 & 94 & Pu & 239 & 311.69 & $2.740 E-07$ & 129.28 & 375.02 \\
\hline 213 & 95 & AM & 241 & 311.94 & $3.900 E-07$ & 59.54 & 103.00 \\
\hline 214 & 94 & Pu & 239 & 313.50 & $2.000 E-08$ & 129.28 & 375.02 \\
\hline 215 & 94 & $P U$ & 239 & 316.35 & $1.410 E-07$ & 375.02 & 413.69 \\
\hline 216 & 94 & PU & 239 & 320.81 & $5.690 E-07$ & 129.28 & 375.02 \\
\hline
\end{tabular}




\section{NUCLIDE}

\begin{tabular}{|c|c|c|c|c|c|}
\hline 217 & 95 & $A M$ & 241 & 322.48 & $1.440 E-06$ \\
\hline 218 & 94 & PU & 239 & 323.76 & $5.390 E-07$ \\
\hline 219 & 92 & u & 237 & 332.29 & $1.140 E-02$ \\
\hline 220 & 95 & $A M$ & 241 & 332.33 & $1.450 E-06$ \\
\hline 221 & 94 & pu & 239 & 332.80 & $5.050 E-26$ \\
\hline 222 & 92 & $u$ & 237 & 335.33 & $1.100 E-03$ \\
\hline 223 & 85 & $A M$ & 241 & 335.38 & $4.700 E-06$ \\
\hline 224 & 94 & PU & 239 & 336.06 & $1.130 E-06$ \\
\hline 225 & 95 & $A M$ & 241 & 337.69 & $3.050 E-08$ \\
\hline 226 & 95 & $A M$ & 241 & 340.40 & $2.500 E-08$ \\
\hline 227 & 94 & PU & 239 & 341.48 & $6.630 E-07$ \\
\hline 228 & 94 & $P U$ & 239 & 344.96 & $5.610 E-06$ \\
\hline 229 & 92 & $u$ & 235 & 345.89 & $3.000 E-04$ \\
\hline 230 & 95 & $A M$ & 241 & 350.40 & $1.400 E-08$ \\
\hline 231 & 94 & $P U$ & 239 & 354.10 & $8.000 E-09$ \\
\hline 232 & 95 & $A M$ & 241 & $35 e .20$ & $1.510 E-08$ \\
\hline 233 & 94 & Pu & 239 & .361 .90 & $1.170 E-07$ \\
\hline 234 & 94 & PU & 239 & 367.02 & $8.720 E-07$ \\
\hline 235 & 94 & PU & 239 & 368.53 & $8.960 E-07$ \\
\hline 236 & 95 & $A M$ & 241 & 368.60 & $2.080 E-06$ \\
\hline 237 & 92 & $\mathbf{u}$ & 237 & 368.70 & $4.300 E-04$ \\
\hline 238 & 92 & $u$ & 238 & 369.50 & $4.800 E-05$ \\
\hline 239 & 92 & $\mathbf{u}$ & 237 & 370.80 & $1.050 E-03$ \\
\hline 240 & 95 & $A M$ & 241 & 370.91 & $4.910 E-07$ \\
\hline 241 & 92 & $u$ & 238 & 371.90 & $2.300 E-05$ \\
\hline 242 & 94 & PU & 239 & 375.02 & $1.585 \varepsilon-05$ \\
\hline 243 & 95 & AM & 241 & 376.57 & $1.330 E-C 6$ \\
\hline
\end{tabular}

ASSCCIATIVE GAMMA RAYS

$\begin{array}{rr}59.54 & 103.00 \\ 129.28 & 375.02 \\ 164.59 & 207.97 \\ 59.54 & 103.00 \\ 375.02 & 413.69 \\ 164.59 & 207.97 \\ 59.54 & 103.00 \\ 375.02 & 413.69 \\ 59.54 & 103.00 \\ 59.54 & 103.00 \\ 375.02 & 413.69 \\ 375.02 & 413.69 \\ 185.72 & 163.37 \\ 59.54 & 103.00 \\ 375.02 & 413.69 \\ 59.54 & 103.61 \\ 129.28 & 375.02 \\ 375.02 & 413.69 \\ 375.02 & 413.69 \\ 59.54 & 103.00 \\ 164.59 & 207.57 \\ 766.39 & 1001.10 \\ 164.59 & 207.97 \\ 59.54 & 103.00 \\ 766.39 & 1001.10 \\ 344.96 & 413.69 \\ 59.54 & 103.00\end{array}$




\begin{tabular}{|c|c|c|c|c|c|c|c|}
\hline & & UCLII & I DE & $\begin{array}{l}\text { ENERGY } \\
\text { (KEV) }\end{array}$ & BR ANCH I NG & ASSCCIATIVE & GAMMA RAY \\
\hline 244 & 94 & $P U$ & 239 & 380.16 & $3.075 E-06$ & 375.02 & 413.69 \\
\hline 245 & 94 & PU & 239 & 382.70 & $2.600 E-06$ & 375.02 & 413.69 \\
\hline 246 & 95 & $A M$ & 241 & 383.74 & $2.900 E-07$ & 59.54 & $125 \cdot 30$ \\
\hline 247 & 92 & U & 235 & 387.81 & $1.200 E-04$ & 185.72 & 163.37 \\
\hline 248 & 95 & $A M$ & 241 & 390.53 & $6.100 E-08$ & 59.54 & $-125: 30$ \\
\hline 249 & 94 & PU & 239 & 392.37 & $1.160 E-06$ & 375.02 & 413.69 \\
\hline 250 & 94 & PU & 239 & 393.04 & $4.440 E-06$ & 375.02 & 413.69 \\
\hline 251 & 95 & $A M$ & 241 & 398.50 & $1.500 E-08$ & 59.54 & 125.30 \\
\hline 232 & 94 & PU & 239 & 399.44 & $6.130 E-08$ & 375.02 & 413.69 \\
\hline 253 & 95 & $A M$ & 241 & 406.46 & $1.200 E-08$ & 59.54 & $125 \cdot 30$ \\
\hline 254 & 94 & PU & 239 & 410.77 & $8.000 E-08$ & 375.02 & 413.69 \\
\hline 255 & 94 & PU & 239 & 413.69 & $1.506 \mathrm{E}-05$ & 375.02 & 393.04 \\
\hline 256 & 95 & $A M$ & 241 & 415.90 & $1.610 E-08$ & 59.54 & $125 \cdot 30$ \\
\hline 257 & 95 & Ain & 241 & 419.19 & $2.760 E-07$ & 59.54 & 125.30 \\
\hline 258 & 94 & PU & 239 & 422.57 & $1.190 E-06$ & 375.02 & $\$ 13.69$ \\
\hline 259 & 95 & $A M$ & 241 & 426.34 & $2.400 E-07$ & 59.54 & 225.30 \\
\hline 260 & 94 & PU & 239 & 426.67 & $2.290 E-07$ & 129.28 & 375.02 \\
\hline 261 & 95 & $A M$ & 241 & 429.60 & $5.000 E-09$ & 59.54 & 125.30 \\
\hline 262 & 94 & $\mathbf{P U}$ & 239 & 430.00 & $4.910 E-08$ & 375.02 & 413.69 \\
\hline 263 & 95 & $A M$ & 241 & 442.75 & $3.400 E-08$ & 59.54 & 125.30 \\
\hline 264 & 94 & $P U$ & 239 & 445.78 & $9.100 E-08$ & 375.02 & 413.69 \\
\hline 265 & 92 & $\mathbf{u}$ & 238 & 451.00 & $3.500 E-05$ & 766.39 & 1001.10 \\
\hline 266 & 94 & $P U$ & 239 & 451.45 & $1.920 E-06$ & 375.02 & 413.69 \\
\hline 267 & 95 & $A M$ & 241 & $452 \cdot 23$ & $1.700 E-08$ & 59.54 & $125 \cdot 30$ \\
\hline 268 & 90 & $T H$ & 228 & 452.90 & $3.900 E-03$ & 238.62 & 583.15 \\
\hline 269 & 92 & $\mathbf{u}$ & 238 & 453.90 & $2.100 E-05$ & 766.39 & 1001.10 \\
\hline 270 & 95 & AM & 241 & 454.58 & 9. $300 E-00$ & 59.54 & 125.30 \\
\hline
\end{tabular}




\begin{tabular}{|c|c|c|c|c|c|c|c|}
\hline \multirow[b]{2}{*}{271} & \multicolumn{3}{|c|}{ NUCLIDE } & \multirow{2}{*}{$\begin{array}{l}\text { ENERSY } \\
\text { (KEV) } \\
457.57\end{array}$} & \multirow{2}{*}{$\begin{array}{l}\text { BRANCHING } \\
\text { INTENSITY } \\
1.540 E-08\end{array}$} & \multirow{2}{*}{$\begin{array}{c}\text { ASSOCIAT IVE } \\
375.02\end{array}$} & \multirow{2}{*}{$\begin{array}{r}\text { GAMPA RAI } \\
413.69\end{array}$} \\
\hline & 94 & PU & 239 & & & & \\
\hline 272 & 92 & $\mathbf{u}$ & 238 & 458.20 & $2.200 E-05$ & 766.39 & $1001 \cdot 10$ \\
\hline 273 & 95 & AM & 241 & 459.40 & $3.200 E-08$ & 59.54 & 125.30 \\
\hline 274 & 94 & PU & 239 & 461.29 & $2.020 E-08$ & 375.02 & 413.69 \\
\hline 275 & 94 & PU & 239 & 463.80 & $1.570 E-09$ & 375.02 & 413.69 \\
\hline 276 & 95 & $A M$ & 241 & 467.90 & $2.500 \varepsilon-08$ & 59.54 & $125 \cdot 30$ \\
\hline 277 & 92 & $u$ & 238 & 468.30 & $1.960 E-05$ & 766.39 & $1001 \cdot 10$ \\
\hline 278 & 94 & PU & 239 & 474.40 & S. $420 E-10$. & 375.02 & 413.69 \\
\hline 279 & 92 & $u$ & 238 & 475.80 & $1.73 O E-05$ & 766.39 & 1001.10 \\
\hline 280 & 94 & PU & 239 & 481.55 & $4.770 E-08$ & 375.02 & 413.69 \\
\hline 281 & 95 & $A M$ & 241 & 486.30 & $1.200 \mathrm{E}-08$ & 59.54 & 125.30 \\
\hline 282 & 94 & PU & 239 & 487.00 & $2.500 E-09$ & 375.02 & 413.69 \\
\hline 283 & 94 & $P U$ & 239 & 493.10 & $8.800 E-09$ & 375.02 & 413.69 \\
\hline 284 & 90 & IH & 228 & 510.73 & $9.500 E-02$ & 238.62 & 583.15 \\
\hline 285 & 95 & $A M$ & 241 & 514.10 & $2.700 E-0 B$ & 59.54 & 125.30 \\
\hline 286 & 95 & $A M$ & 241 & 521.90 & $7.000 E-09$ & 59.54 & 125.30 \\
\hline 287 & 94 & $P U$ & 239 & 538.90 & $3.000 E-09$ & 375.02 & 413.69 \\
\hline 288 & 92 & $u$ & 238 & 544.00 & $3.500 E-05$ & 766.39 & $2001 \cdot 10$ \\
\hline 289 & 94 & PU & 239 & 550.60 & $4.000 E-09$ & 375.02 & 413.69 \\
\hline 290 & 94 & PU & 239 & 557.70 & $6.000 E-10$ & 375.02 & $\$ 13.69$ \\
\hline 291 & 92 & $\mathbf{u}$ & 238 & 569.29 & $2.020 E-04$ & 766.39 & 1001.10 \\
\hline 392 & 95 & $A M$ & 241 & 574.00 & $1.450 E-08$ & 59.54 & 125.30 \\
\hline 293 & 90 & $r_{H}$ & 228 & 583.15 & $3.100 E-01$ & 238.62 & 2614.71 \\
\hline 294 & 94 & PU & 239 & 586.40 & $1.420 E-09$ & 375.02 & 413.69 \\
\hline 295 & 95 & $A M$ & 241 & 586.52 & $1.430 E-08$ & 59.54 & 125.30 \\
\hline 296 & 95 & AM & 241 & 590.30 & $1.520 E-08$ & 59.54 & 125.30 \\
\hline 297 & 95 & AM & 241 & 597.39 & $6.500 E-08$ & 59.54 & 225.39 \\
\hline
\end{tabular}




\begin{tabular}{|c|c|c|c|c|c|c|c|}
\hline & & UCLI & IDE & $\begin{array}{l}\text { ENERGY } \\
\text { (KEV) }\end{array}$ & $\begin{array}{l}\text { BRANCHING } \\
\text { INTENSI TY }\end{array}$ & ASSOCIATIVE & GAMNA RAY \\
\hline 298 & 94 & $P U$ & 239 & 598.10 & $1.900 E-08$ & 375.02 & 413.69 \\
\hline 299 & 94 & $\mathrm{PU}$ & 239 & 607.30 & $1.450 E-09$ & 375.02 & 413.69 \\
\hline 300 & 94 & $\mathrm{PU}$ & 239 & 612.90 & 8. 1 DOE-09 & 375.02 & 413.69 \\
\hline 301 & $9 ;$ & PU & 239 & 617.40 & $2.040 E-08$ & 375.02 & $\$ 13.69$ \\
\hline 302 & 94 & PU & 239 & 618.90 & $2.440 E-08$ & 375.02 & 413.69 \\
\hline 303 & 95 & $A M$ & 241 & 618.96 & $5.550 \mathrm{E} \cdot 07$ & 662.37 & 721.92 \\
\hline 304 & 94 & PU & 239 & 624.80 & $4.000 E-09$ & 375.02 & 413.69 \\
\hline 305 & 95 & $A M$ & 241 & 626.90 & $3.600 E-09$ & 662.37 & 721.92 \\
\hline 306 & 95 & $A M$ & 241 & 532.80 & $1.100 E-08$ & 662.37 & 721.92 \\
\hline 307 & 94 & $P U$ & 239 & 633.19 & $2.320 E-08$ & 375.02 & 413.69 \\
\hline 308 & 24 & PU & 239 & 637.97 & $2.500 E-08$ & 375.02 & 413.69 \\
\hline 309 & 94 & PU & 239 & 640.15 & $7.950 E-08$ & 375.02 & 413.69 \\
\hline 310 & 95 & $A M$ & 241 & $641 \cdot 37$ & $6.450 E-0 B$ & 662.37 & 721.92 \\
\hline 311 & 94 & PU & 240 & $642 \cdot 30$ & $1.450 E-07$ & 160.35 & 687.63 \\
\hline 312 & 94 & PU & 239 & 646.02 & $1.450 E-07$ & 375.02 & 423.69 \\
\hline 313 & 94 & PU & 239 & 649.50 & $8.100 E-09$ & 375.02 & 413.69 \\
\hline 314 & 94 & PU & 239 & 652.19 & $6.400 E-08$ & 375.02 & 413.69 \\
\hline 315 & 95 & $A M$ & 241 & 652.88 & $3.540 E-07$ & 662.37 & 721.92 \\
\hline 316 & 92 & $\mathbf{u}$ & 238 & $654 \cdot 30$ & $2.160 E-05$ & 766.39 & $1001 \cdot 10$ \\
\hline 317 & 94 & PU & 239 & 654.86 & $2.150 E-08$ & 375.02 & $\$ 13.69$ \\
\hline 318 & 94 & PU & 239 & 658.99 & $9.500 E-08$ & 375.02 & 413.69 \\
\hline 319 & 95 & $A M$ & 241 & 662.37 & $3.46 C E-06$ & 207.98 & 721.92 \\
\hline 320 & 94 & PU & 239 & 664.67 & $1.54 O E-08$ & 375.02 & 413.69 \\
\hline 321 & 94 & PU & 239 & 674.20 & $5.200 E-09$ & 375.02 & 413.69 \\
\hline 322 & 95 & AN & 241 & 675.70 & $6.800 E-09$ & 662.37 & 721.92 \\
\hline 323 & 95 & AM & 241 & 679.99 & 2. $\triangle 00 E-08$ & 662.37 & 721.92 \\
\hline 324 & 94 & PU & 239 & 686.16 & $8.900 E-09$ & 375.02 & $\$ 13.69$ \\
\hline
\end{tabular}




\begin{tabular}{|c|c|c|c|c|c|c|c|}
\hline & & UCLI & I DE & $\begin{array}{l}\text { ENERGY } \\
\text { (KEV) }\end{array}$ & $\begin{array}{l}\text { BRANCH I NG } \\
\text { INTENSITY }\end{array}$ & ASSOCIATIVE & GAMMA RAY \\
\hline 325 & 94 & PU & 240 & 687.63 & $3.700 E-08$ & 160.35 & 642.30 \\
\hline 326 & 95 & AM & 241 & 688.70 & $2.920 E-07$ & 662.37 & 721.92 \\
\hline 327 & 94 & $P U$ & 239 & 590.85 & $5.500 E-09$ & 375.02 & 413.69 \\
\hline 328 & 92 & u & 238 & 691.30 & $9.000 E=05$ & 766.39 & $1001 \cdot 10$ \\
\hline 329 & 95 & $A M$ & 241 & 693.41 & $3.160 E-08$ & 662.37 & 721.92 \\
\hline 330 & 95 & $A M$ & 241 & 696.34 & $4.900 E-08$ & 662.37 & 721.92 \\
\hline 331 & 92 & u & 238 & 699.00 & $6.000 E-05$ & 766.39 & $1001 \cdot 10$ \\
\hline 332 & 94 & FU & 239 & 701.00 & $5.400 E-09$ & 375.02 & 413.69 \\
\hline 333 & 92 & U & 238 & $702 \cdot 10$ & $7.000 E-05$ & 766.39 & 1001.10 \\
\hline 334 & 94 & PU & 239 & 703.79 & $3.880 E-08$ & 375.02 & 413.69 \\
\hline 335 & 94 & PU & 238 & 705.60 & $5.400 E-10$ & 152.77 & 766.41 \\
\hline 336 & 92 & $\mathbf{u}$ & 238 & 706.00 & $7.000 E-05$ & 766.39 & $1001 \cdot 10$ \\
\hline 337 & 94 & PU & 238 & 708.40 & $5.380 E-09$ & 152.77 & 766.41 \\
\hline 338 & 95 & $A M$ & 241 & 709.30 & $5.800 E-08$ & 662.37 & $7.21 .9 ?$ \\
\hline 339 & 94 & PU & 239 & 717.76 & $2.670 E-08$ & 375.02 & 413.69 \\
\hline 340 & 95 & $A M$ & 241 & $721 \cdot 92$ & $1.850 E-06$ & 662.37 & 207.98 \\
\hline 341 & 90 & TH & 228 & $727 \cdot 26$ & $6.800 E-02$ & 238.62 & 583.15 \\
\hline 342 & 94 & PU & 239 & 727.81 & $1.090 E-09$ & 375.02 & 413.69 \\
\hline 343 & 95 & $A M$ & 241 & 729.46 & $1.300 E-08$ & 662.37 & 721.92 \\
\hline 344 & 92 & $\mathbf{u}$ & 238 & 733.39 & $1.060 E-04$ & 766.39 & $1001 \cdot 10$ \\
\hline 345 & 95 & $A M$ & 241 & 737.22 & $7.500 E-08$ & 662.37 & 721.92 \\
\hline 346 & 92 & $\mathbf{u}$ & 238 & 739.70 & $1.100 E-04$ & 766.39 & 1001.10 \\
\hline 347 & 92 & $\mathbf{u}$ & 238 & 742.79 & $9.500 E-04$ & 766.39 & 1001.10 \\
\hline 348 & 94 & PU & 238 & 742.82 & $5.550 E-08$ & 152.77 & 766.41 \\
\hline 349 & 95 & $A M$ & 241 & 755.87 & $7.100 E-08$ & 662.37 & 721.92 \\
\hline 350 & 94 & PU & 239 & 756.40 & $3 \cdot 370 E-08$ & 375.02 & 413.69 \\
\hline 351 & 95 & $A M$ & 241 & 759.37 & $1.650 E-08$ & 662.37 & 721.92 \\
\hline
\end{tabular}




\begin{tabular}{|c|c|c|c|c|c|c|c|}
\hline & & SCLIO & IOE & $\begin{array}{l}\text { ¿NERGY } \\
\text { (KEV) }\end{array}$ & $\begin{array}{l}\text { ARANCHING } \\
\text { INTENSITY }\end{array}$ & ASSCC IAT IVE & GAMNA RA \\
\hline 352 & 90 & TH & 228 & 763.30 & $6.600 E-03$ & 238.62 & 583.15 \\
\hline 353 & 92 & $u$ & 238 & 766.39 & $3.130 E-03$ & 742.79 & $1001 \cdot 10$ \\
\hline 354 & 94 & PU & 238 & 766.41 & $2.400 E-07$ & 152.77 & 742.82 \\
\hline 355 & 95 & $A M$ & 241 & 766.87 & $5.100 E-08^{\circ}$ & 662.37 & 721.92 \\
\hline 356 & 94 & PU & 239 & 769.38 & $1.100 E-07$ & 375.02 & 413.69 \\
\hline 357 & 95 & $A M$ & 241 & 770.93 & $6.47 O E-O B$ & 662.37 & 721.92 \\
\hline 358 & 94 & $P:$ & 239 & 779.50 & $1.330 E-09$ & 375.02 & 413.69 \\
\hline 359 & 95 & $A M$ & 241 & 780.42 & $2.700 E-09$ & 662.37 & 721.92 \\
\hline 360 & 92 & $u$ & 238 & 781.50 & $7.000 E-05$ & 766.39 & 1001.10 \\
\hline 361 & 90 & TH & 228 & 785.46 & $1.150 E-02$ & 238.62 & 583.15 \\
\hline 362 & 94 & PU & 238 & 786.30 & $3.530 E-08$ & 152.77 & 766.41 \\
\hline 363 & 92 & $u$ & 238 & 786.30 & $5.500 E-04$ & 766.39 & $1001 \cdot 10$ \\
\hline 364 & 94 & PU & 239 & 787.21 & $8.400 E-10$ & 375.02 & 413.69 \\
\hline 365 & 94 & PU & 239 & 193.00 & $2.500 E-10$ & 375.02 & 413.69 \\
\hline 366 & 92 & U & 238 & 796.40 & $6.650 E-05$ & 766.39 & $1001 \cdot 10$ \\
\hline 367 & 94 & Pu & 239 & 796.50 & $3.200 E-10$ & 375.02 & 413.69 \\
\hline 368 & 94 & PU & 239 & 803.28 & $4.450 \subseteq-10$ & 375.02 & 413.69 \\
\hline 369 & 94 & PU: & 238 & 805.42 & $1.390 E-09$ & 252.77 & 266.41 \\
\hline 370 & 92 & $\|$ & 238 & 805.80 & $7.600 E-05$ & 766.39 & $1001 \cdot: 30$ \\
\hline 371 & 94 & PU 2 & 239 & 808.14 & $1.470 E-09$ & 375.02 & 413.69 \\
\hline 372 & 94 & PU: & 238 & 808.23 & B. 580E-09 & 152.77 & $2.66 \cdot 41$ \\
\hline 373 & 92 & $u 2$ & 238 & 808.40 & $2.870 E-05$ & 766.39 & 1001.10 \\
\hline 374 & 95 & AM 2 & 241 & 811.80 & $5.200 E-09$ & 662.37 & 7.21 .92 \\
\hline 375 & 94 & $P u 2$ & 239 & 813.89 & $6.20 C E-10$ & 375.02 & 413.69 \\
\hline 376 & 92 & $\mathbf{U} 2$ & 238 & 819.10 & $2.300 E-05$ & 766.39 & $1001 \cdot 10$ \\
\hline 377 & 94 & PU 2 & 239 & 821.02 & $5.110 E-10$ & 375.02 & 413.69 \\
\hline 378 & 92 & U & 238 & 825.00 & $6.000 E-05$ & 766.39 & 1001.10 \\
\hline
\end{tabular}




\begin{tabular}{|c|c|c|c|c|c|c|c|}
\hline & & UCLI & IDE & $\begin{array}{l}\text { ENERGY } \\
\text { (KEV) }\end{array}$ & $\begin{array}{l}\text { ERA ACH ING } \\
\text { I NTEINS I TY }\end{array}$ & ASSCCIATIVE & GAMHA RAY \\
\hline 379 & 94 & PU & 239 & 828.61 & $1.380 E-09$ & 375.02 & 413.69 \\
\hline 380 & 92 & $\mathbf{U}$ & 238 & 831.30 & $7.400 E-05$ & 766.39 & $1001 \cdot 10$ \\
\hline 381 & 94 & PU & 239 & 832.06 & $2.540 E-10$ & 375.02 & 413.69 \\
\hline 382 & 94 & $\mathbf{P U}$ & 239 & $840 . \mathrm{CO}$ & $2.800 E-10$ & 375.02 & 413.69 \\
\hline 383 & 94 & $P U$ & 239 & 843.75 & $1.500 E-09$ & 375.02 & 413.69 \\
\hline 384 & 92 & u & 238 & 851.66 & $7.400 E-05$ & 766.39 & $1001 \cdot 10$ \\
\hline 385 & 94 & PU & 238 & 851.73 & $1.390 E-08$ & 152.77 & 766.41 \\
\hline 386 & 95 & $A M$ & 241 & 851.90 & $2.800 E-09$ & 662.37 & 721.92 \\
\hline 387 & 90 & $T H$ & 228 & 860.49 & $4.650 E-02$ & $238 . \epsilon 2$ & 583.15 \\
\hline 388 & 95 & AM & 241 & 862.90 & $5.100 E-09$ & 662.37 & 721.92 \\
\hline 389 & 92 & $u$ & 238 & 866.50 & $1.200 E-05$ & 766.39 & $1001 \cdot 10$ \\
\hline 390 & 92 & $u$ & 238 & 876.10 & $4.000 E-05$ & 766.39 & $1001 \cdot 10$ \\
\hline 391 & 94 & $P U$ & 239 & 878.96 & $5.110 E-10$ & 375.02 & 413.69 \\
\hline 392 & 92 & $\mathbf{u}$ & 238 & 880.50 & $1.960 E-04$ & 766.39 & $1001 \cdot 10$ \\
\hline 393 & 94 & PU & 238 & 883.21 & $6.530 E-09$ & 152.77 & 766.41 \\
\hline 394 & 92 & $\mathbf{u}$ & 238 & 383.24 & $1.970 E-04$ & 766.39 & 1001.10 \\
\hline 395 & 92 & $\mathbf{u}$ & 238 & 887.39 & $6.300 E-05$ & 766.39 & $1601 \cdot 10$ \\
\hline 396 & 94 & PU & 239 & 891.00 & $7.760 E-10$ & 375.02 & 413.69 \\
\hline 397 & 90 & TH & 228 & 893.33 & $3.7005-03$ & 238.62 & 583.15 \\
\hline 398 & 92 & $u$ & 238 & 898.67 & $4.500 E-05$ & 766 × 39 & $1001 \cdot 10$ \\
\hline 399 & $9 \%$ & PU & 238 & 904.34 & $6.210 E-10$ & 152.77 & $7 \in 6.41$ \\
\hline 400 & 92 & $u$ & 238 & 921.77 & $1.250 E-04$ & 766.39 & 1001.10 \\
\hline 401 & 92 & $u$ & 238 & 925.07 & $1.360 E-04$ & 766.39 & $1001 \cdot 10$ \\
\hline 402 & 92 & $\mathbf{u}$ & 238 & 926.68 & $1.910 E-04$ & 766.39 & $1001 \cdot 10$ \\
\hline 403 & 94 & PU & 238 & 926.73 & $6.150 E-09$ & 152.77 & 766.41 \\
\hline 404 & 92 & $u$ & 238 & 936.70 & $7.500 E-06$ & 766.39 & $1001 \cdot 10$ \\
\hline 405 & 94 & PU & 239 & 940.00 & $4.200 E-10$ & 375.02 & 413.69 \\
\hline
\end{tabular}




\begin{tabular}{|c|c|c|c|c|c|c|c|}
\hline \multirow[b]{2}{*}{406} & \multicolumn{3}{|c|}{ NUCLIDE } & \multirow{2}{*}{$\begin{array}{l}\text { ENERGY } \\
\text { (KEV) } \\
941.50\end{array}$} & \multirow{2}{*}{$\begin{array}{l}\text { BRANCHI NG } \\
\text { INTENSI TY } \\
2.400 E-05\end{array}$} & \multirow{2}{*}{$\begin{array}{c}\text { ASSOCIAT IVE } \\
766.39\end{array}$} & \multirow{2}{*}{$\begin{array}{l}\text { GAMMA RA } \\
1001.10\end{array}$} \\
\hline & 92 & $u$ & 238 & & & & \\
\hline 407 & 94 & PU & 238 & 942.02 & $5.260 E-09$ & 152.77 & 766.41 \\
\hline 408 & 92 & $u$ & 238 & 946.07 & $3.550 E-04$ & 766.39 & 1001.10 \\
\hline 409 & 94 & PU & 238 & 946.12 & $1.030 E-09$ & 152.77 & 766.41 \\
\hline 410 & 94 & PU & 239 & 956.40 & $5.600 E-10$ & 375.02 & 413.69 \\
\hline 411 & 92 & $u$ & 238 & 965.71 & $5.650 E-06$ & 766.39 & 1001.10 \\
\hline 412 & 94 & PU & 239 & 979.50 & $2.260 E-10$ & 375.02 & 413.69 \\
\hline 413 & 92 & $u$ & 238 & 580.50 & $4.200 E-05$ & 766.39 & $1001 \cdot 10$ \\
\hline 414 & 92 & $u$ & 238 & 984.10 & $2.700 E-05$ & 766.39 & $1001 \cdot 10$ \\
\hline 415 & 94 & PU & 239 & 986.70 & $1.200 E-10$ & 375.02 & 413.69 \\
\hline 416 & 94 & Pu & 239 & 992.40 & $2.260 E-10$ & 375.02 & 413.69 \\
\hline 417 & 92 & $u$ & 238 & 994.90 & $7.480 E-05$ & 766.39 & $1001 \cdot 10$ \\
\hline 418 & 92 & $u$ & 238. & $1002 \cdot 10$ & $8.280 E-03$ & 766.39 & 742.79 \\
\hline 419 & 94 & PU & 238 & 1001.20 & $1.300 E-08$ & 152.77 & 766.41 \\
\hline 420 & 94 & PU & 239 & 1005.50 & $1.200 E-10$ & 375.02 & $\$ 13.69$ \\
\hline 421 & 92 & $\mathbf{u}$ & 238 & 1028.70 & $5.540 E-06$ & 766.39 & $1001 \cdot 10$ \\
\hline 422 & 92 & $u$ & 238 & 1041.70 & $5.580 E-06$ & 766.39 & $1001 \cdot 10$ \\
\hline 423 & 94 & PU & 238 & 1041.90 & $2.290 E-09$ & 152.77 & 766.41 \\
\hline 424 & 92 & $\mathbf{u}$ & 238 & 1044.30 & $7.460 E-06$ & 766.39 & $1001 \cdot 10$ \\
\hline 425 & 94 & pu & 239 & 1057.30 & $4.500 E-10$ & 375.02 & 413.69 \\
\hline 426 & 92 & $u$ & 238 & 2061.86 & $2.400 E-05$ & 766.39 & 1001.10 \\
\hline 427 & 90 & TH & 228 & 2078.70 & $5.500 E-03$ & 230.62 & 583.15 \\
\hline 428 & 92 & $u$ & 238 & 1083.70 & $1.600 E-05$ & 766.39 & $1001 \cdot 10$ \\
\hline 429 & 94 & Pu & 238 & 1085.40 & $8.850 E-10$ & 152.77 & 766.41 \\
\hline 430 & 92 & $u$ & 238 & 1122.00 & $6.530 E-06$ & 766.39 & $1001 \cdot 10$ \\
\hline 431 & 92 & $u$ & 238 & 1125.10 & $4.700 E-05$ & 766.39 & 1001.10 \\
\hline 432 & 92 & $u$ & 238 & 1151.10 & $3.900 E-06$ & 766.39 & $1001 \cdot 10$ \\
\hline
\end{tabular}




\begin{tabular}{|c|c|c|c|c|c|c|c|}
\hline \multirow[b]{2}{*}{433} & \multicolumn{3}{|c|}{ NUCLIDE } & \multirow{2}{*}{$\begin{array}{l}\text { ENERGY } \\
\text { (KEV) } \\
1193.76\end{array}$} & \multirow{2}{*}{$\begin{array}{l}\text { BRANCHING } \\
\text { INTENSITY } \\
1.340 E-05\end{array}$} & \multirow{2}{*}{$\begin{array}{c}\text { ASSOCIATIVE } \\
766.39\end{array}$} & \multirow{2}{*}{$\begin{array}{l}\text { GAMMA RAY } \\
1001.10\end{array}$} \\
\hline & 92 & $\mathbf{u}$ & 238 & & & & \\
\hline 434 & 92 & $\mathbf{U}$ & 238 & 1220.10 & $3.750 E-06$ & 766.39 & 1001.10 \\
\hline 435 & 92 & $\mathbf{u}$ & 238 & 1237.26 & $4.900 E-05$ & 766.39 & $1001 \cdot 10$ \\
\hline 436 & 92 & $u$ & 238 & 1292.70 & $8.600 E-06$ & 766.39 & $1001 \cdot 10$ \\
\hline 437 & 92 & u & 238 & 1320.50 & $2.900 E-06$ & 766.39 & $1001 \cdot 10$ \\
\hline 438 & 92 & $u$ & 238 & 1352.90 & $2.240 E-05$ & 766.39 & $1001 \cdot 10$ \\
\hline 439 & 92 & $u$ & 238 & 1393.50 & $3.700 E-05$ & 766.39 & 2001.10 \\
\hline 440 & 92 & $u$ & 238 & 1413.90 & $2.310 E-05$ & 766.39 & $1001 \cdot 10$ \\
\hline 441 & 92 & $u$ & 238 & 1434.27 & $9.000 E-05$ & 766.39 & $1001 \cdot 10$ \\
\hline 442 & 92 & $u$ & 238 & 1445.80 & $4.000 E-06$ & 766.39 & $1001 \cdot 10$ \\
\hline 443 & 92 & $u$ & 238 & 1452.60 & $9.500 E-06$ & 766.39 & $1001 \cdot 10$ \\
\hline 444 & 92 & $u$ & 238 & 1510.32 & 1. $300 E-04$ & 766.39 & 1001.10 \\
\hline 445 & 92 & $\mathbf{u}$ & 238 & 1527.25 & $2.180 E-05$ & 766.39 & $1001 \cdot 10$ \\
\hline 446 & 92 & $\mathbf{u}$ & 238 & 1548.24 & $1.400 E-05$ & 766.39 & 1001.10 \\
\hline 447 & 92 & $u$ & 238 & 1553.80 & $8.200 E-05$ & 766.39 & $1001 \cdot 10$ \\
\hline 448 & 92 & $u$ & 238 & 1558.90 & $7.000 E-0 E$ & 766.39 & $1001 \cdot 10$ \\
\hline 449 & 92 & u & 238 & 1570.80 & $1.050 E-05$ & 766.39 & 1001.10 \\
\hline 450 & 90 & TH & 228 & 1592.69 & PAIR PEAK & 238.62 & 583.15 \\
\hline 451 & 92 & $u$ & 238 & 1593.20 & $3.400 E-06$ & 766.39 & $1001 \cdot 10$ \\
\hline 452 & 92 & $u$ & 238 & 1593.20 & $2.650 E-05$ & 766.39 & $1001 \cdot 20$ \\
\hline 453 & 90 & TH & 228 & 1620.63 & $1.540 E-02$ & 238.62 & 583.15 \\
\hline 454 & 92 & $u$ & 238 & 1638.10 & 3. $800 E-06$ & 766.39 & $1001 \cdot 10$ \\
\hline 455 & 92 & $\mathbf{u}$ & 238 & $1654 \cdot 10$ & $3.300 E-06$ & 766.39 & $1001 \cdot 10$ \\
\hline 456 & 92 & $u$ & 238 & 1668.40 & $1.200 E-05$ & 766.39 & $1001 \cdot 10$ \\
\hline 457 & 92 & $\mathbf{u}$ & 238 & 1685.80 & $2.970 E-06$ & 766.39 & 1001.10 \\
\hline 458 & 92 & $\mathbf{u}$ & 238 & 1694.30 & $1.280 E-05$ & 766.39 & $1001 \cdot 10$ \\
\hline 459 & 92 & $u$ & 238 & 1716.50 & $1.940 E-06$ & 766.39 & $1001 \cdot 10$ \\
\hline
\end{tabular}




\section{APPENDIX B}

\section{STATISTICS}

The following is a brief, nonrigorous discussion of the statistical treatment of nondestructive assay data. It is primarily a compilation of useful statistical formulas employed in reporting nuclear assay results. The discussion considers random errors only. There is no consideration of the often serious problem of sysiematic errors (biases). The notation, which often does not conform with modern statistical practice, has been chosen deliberately to convey a few basic ideas without providing the statistical fundamentals. The material in this appendix is not intended as a substitute for sound professional statistical consultation.

\section{General}

1. Consider a group of $\mathbf{N}$ measurements of some physical quantity $X$; e.g., $X=$ grams plutonium as measured on a neutron counter. $\overline{\mathbf{X}}$.

2. The best estimate of $\mathrm{X}$ is the cuerage, or mean,

$$
\bar{x}=\sum_{i=1}^{N} x_{i} / N
$$

3. In general, each measurement, $X_{i}$, deviates from this average. $A$ common indicator of the magnitude of this deviation is the standard deviation $\sigma_{\mathbf{x}_{\mathbf{i}}}$. which is estimated by

$$
u_{x_{i}} \simeq \sqrt{\frac{\sum_{i}^{N}\left(N_{i}-N\right)^{2}}{N-1}}, N>1 .
$$

This may be quoted as the estimated relative siandard deviation.

$$
\sigma_{r}-\sigma_{x_{i}} / \bar{X} \cdot 100 \text { percent }
$$

4. It is usually assumed that the measurements are distributed about the mean according to a Gaussian (or normal) distribution. Assuming this, one can estimate the percentage of the measurements which should lie outside a specified interval abou: the mean.

Width of Region,

\begin{tabular}{c}
$\overline{\mathbf{X}} \pm\left\{\sigma_{\mathbf{x}_{\mathbf{i}}}\right.$ \\
\hline$=0.67$ \\
1.0 \\
2.0 \\
3.0
\end{tabular}

Estimated Percentage of Measurements Outside Region 50 32 5 0.3

5. The estimated standard deviation of the mean $\overline{\mathrm{X}}$ is given by

$$
{ }^{3} \bar{x}=\sigma_{x_{i}} / \sqrt{N}
$$

This indicates an increase in precision from repeated measurements.

There is $\sim 68 \%$ probability that the correct answer lies within the range

$$
\overline{\mathrm{X}}-\sigma_{\mathrm{x}_{i}} / \sqrt{\mathrm{N}}<\mathrm{x}<\overline{\mathrm{X}}+J_{\mathrm{x}_{i}} / \sqrt{\mathrm{N}} \text { (one sigma). }
$$

There is $\sim 95 \%$ probability that the correct answer lies within the range

$$
\bar{x}-20 x_{i} / \sqrt{N}<x<\bar{X}+20 x_{i} / \sqrt{N} \text { (two sigma). }
$$

6. The above expression for $\overline{\mathrm{X}}, o_{\mathrm{x}_{\mathrm{i}}}$, and $\sigma \overline{\mathrm{X}}$ should be used to analyze the results from a series of repeated measurements on a single sumple. 


\section{Propagation of Errors}

1. Often the final answer (grams plutonium) involves several measurements with different uncertainties,

$$
\text { for example, } g \mathrm{Pu}=\mathrm{C}(\mathrm{P}-\mathrm{k} \cdot \mathrm{B}) \text {, }
$$

where

$$
\begin{aligned}
& C=\text { calibration (grams/count), } \\
& P=\text { counts in peak window, } \\
& B=\text { courts in background window, and } \\
& k \text { - constant. }
\end{aligned}
$$

2. There are general formulas to handle most cases, several of the most commonly used expressions are presented here.

\section{Let $x$ have standac deviation $\sigma_{x}$, \\ let $y$ have standard deviation $\sigma_{y}$, and \\ let $\mathbf{k}$ be a constant,}

where $\mathrm{x}$ and $\mathrm{y}$ are independent.

a. If $z \cdot x+y$ or $z x-y$,

$$
\therefore z \sqrt{z^{2}+y^{2}} .
$$

b. If $z=x / y$ or $z=x y$,

$$
\frac{\sigma}{z} \simeq \sqrt{\left(\frac{\sigma}{x}\right)^{2}+\left(\frac{\sigma}{y}\right)^{2}} .
$$

c. If $\mathrm{z}=\mathrm{kx}$,

$$
\sigma_{z}=k \sigma_{x}
$$

3. For the excmple given in 1 cbove,

$$
\begin{aligned}
& z=g P u-C(P-k \cdot 13) . \\
& \frac{\sigma}{z} \simeq \sqrt{\left(\frac{\sigma}{C}\right)^{2}+\frac{\sigma_{P}^{2}+k^{2}{ }_{B}^{2}}{(P-k \cdot B)^{2}}} .
\end{aligned}
$$

\section{Nuclear Counting Statistic}

1. All of the description of parts I and II applies to measurements involving nuclear particle counting.

2. Let $X$ be the actual number of counts from a nwelear counving measurement. It is possible to estimate the standard deviation

$$
\sigma_{x} \approx \sqrt{x}
$$

Therefore, based on a eingle measurement of $X$, there is $\sim 68 \%$ probability that the actual rate is included in the nithrval

$$
(x \pm \sqrt{x}) / t
$$

where $t$ is the count time.

3. Consider Example II.3. Assume that $k=1$ and $\sigma_{\mathrm{C}}=\mathbf{0}$.

$$
\begin{aligned}
& z_{P}(:(P-I 3), \\
& \sigma_{P}=\sqrt{P}, \\
& \sigma_{B} \simeq \sqrt{13}, \text { ind } \\
& \sigma_{Z}=(\sqrt{P+B} .
\end{aligned}
$$

The fractional standard deviation is given as

$$
\frac{\sigma}{z} \simeq \frac{\sqrt{P+B}}{P-B} .
$$

4. If $\mathbf{N}$ mecsurements are made on the same samplas, the relative standard deviation of the mean $z$ (grams plutonium) can be computed from

$$
\frac{\mathrm{J}}{\mathrm{z}} \simeq \frac{\sqrt{\overline{\mathrm{P}}+\overline{\mathrm{B}}}}{\overline{\mathrm{P}}-\overline{\bar{B}}} / \sqrt{\mathrm{N}}
$$

Fo: a large number of repeated measurements or when Eq. (B-8) is not a good approximation (see the following sectiol.! it is better to compute the standard deviation from Eqs. (B-2) and (B-4) with $X_{i}$ replaced by $z_{i}$. 
5. If the counting equipment is functioning properly, the estimated standard deviation of the distribution of repeated measurements [Eq. (B-2)] should agree reasonably well with that predicted from the square root of the number of counts [Eq. (B8)]. If the former is very much larger, one should suspect a possible malfunction in the equipment or change in the sample-instrument configuration.

6. In general, unknowns will be counted only once so that error estimates are based on Eq. (B-8). A standard should be counted several times, therefore Eqs. (B-2) and (B-4) are used here.

7. Note that all of the preceding discussion pertains to the precision or repeatability of measurements. This analysis gives no information regarding the accuracy of a measurement (Is it related to the correct amount of material?).

\section{Example}

1. Consider the following count data obtained from a plutonium sample.

$\begin{array}{rrrr} & \text { P } & \text { B } & \text { P.B } \\ & 104256 & 102374 & 1882 \\ 103643 & 102044 & 1599 \\ & 104211 & 101487 & 2724 \\ & 104638 & 102225 & 2413 \\ & 104534 & 102025 & 2509 \\ & 104044 & 102039 & 2005 \\ \operatorname{mean}[\mathrm{Eq}(\mathrm{B}-1)]= & 104221 & 102032 & 2189 \\ \operatorname{sigma}[\mathrm{Eq} \cdot(\mathrm{B}-2)]= & 357 & 300 & 428\end{array}$

2. Suppose $P$ is directly proportional to grams plutonium (i.e., $B=0$ ). The values of $P$ indicate $x$ very precise measurement. Using Eq. (B-8) the standard deviation of $P$ is estimated as

$$
{ }_{\mathrm{P}}=\sqrt{104221}: 323 \text {, }
$$

which agrees with the value computed from E. (B2). It should be emphasized that the sigma discussed here is the standard deviation of the distribution of individual measurements of $P$, not the standard deviation of the mean $\overline{\mathrm{P}}$ (this is $\sigma_{\mathrm{P}} / \sqrt{6}=146$ ).

3. Suppose (P - B) is proportional to grams plutonium. The values indicate a rather imprecise measurement of (P - B). Equations (B-5) and (B-8) allow us to estimate the standard deviation of the distribution of $\mathbf{P}$ - B.

$$
\sigma_{(P-B)} \simeq \sqrt{\bar{P}+\bar{B}}=454
$$

This is in good agreement with the value computed from Eq. (B-2). Note that both P and B are measured individually 10 better ihan $1 / 2 \%$ in a single measurement. However, the difference is measured to only $20 \%$. This illustrates a very poor counting situction. 


\section{APPENDIX C}

\section{CRITICALITY SAFETY}

Criticality is a result of the neutron fission process, and is characterized by the attainment of $a$ selfsustaining fission chain reaction. A system is said to be just critical if a neutron population, once established, will maintain itself constant. This requires a delicate balance between fission and neutron capture plus leakage. If the introduction rate of neutrons in to the system by fission exceeds the loss of neutrons by capture or by leakage irom the system, the system is supercritical. The results can be devastating.

All parameters that influence neutros behavior in a system will also affect criticality. Therefore criticality depends not only on the quantity of fissile material present (e.g., ${ }^{235} \mathrm{U}$ or ${ }^{239} \mathrm{Pu}$ ), but also on the size and shape of the container, on the nature of any additional material (moderator) present in the container, and on the presence of any adjacent material (including human bodies) which might reflect neutrons back into the container. To illustrate the effect of such parameters on criticality, consider a critical sphere of uranium metal at normal density and enriched to $93 \%$ of ${ }^{235} \mathrm{U}$. Such a sphere has a diameter of $\sim 17.5 \mathrm{~cm}$ and a mass of $\sim 52 \mathrm{~kg}$. If this sphere is immersed in water (or placed near any other material), some of the neutrons that would have leaked from the sphere and escaped the system are reflected back into the sphere, and it becomes supercritical. The critical diameter of the water-reflected uranium sphere drops to $\sim 13.5 \mathrm{~cm}$, and the corresponding uranium mass is $\sim 24.5 \mathrm{~kg}$. If sufficient water is also mixed homogeneously with the uranium, the critical diameter (of the water-reflected sphere) increases to $\sim 31 \mathrm{~cm}$, but the critical mass of ${ }^{235} \mathrm{U}$ is only $\sim 0.8 \mathrm{~kg}$. These last conditions are of special interest in that ther represent the minimum critical mass of ${ }^{235} \mathrm{U}$ encountered in usual processes.

The nondestructive assay of fissile maierial by either active or passive techniques often involves placing the container inio a highly reflecting coun. ting geometry. In particular, neutron counters are often embedded in a relatively thick polyethylene moderator. Although this combination is usually not sufficiently well coupled to the sample to constitute a full reflector," neither is it a minimum reflector.

* A full reflector is usually considered to be an unlimited quantity of water surrounding the sysiem.
Although an individual container is expected to contain only a relatively small amount of fissile material, the assayist must be ceriain that it carnot inadvertently contain sufficient material to present a criticality safety hazard when placed into the counter. This is particularly a problem for the assay of unsegregated scrap or waste for which there is little or no reliable information on the annount or enrichment of the fissionable material or on the nature of the matrix material present. Past experience has shown that the tag information on a container of scrap is not always reliable. We have found enrichments nearly double those staled, "dry" scrap that sloshes when handied, and omounts of fissile material an order of magnitude greater than expected. Often, the only information available is the volume and gross weight of the container. This may be sufficient. The conservative Issumption is that the assay system constitutes a fully reflected configuration, and a weight limit is established* sufficiently low so that the given volume could not contain a critical combination of fissile material and optimum moderator. If this is not possible-that is, if the containers to be assayed are too heavy-there are several possible ways to arrive at critically safe operating limits. Multiplication measurements may be made in place ${ }^{C-1}$ in the assay system or detailed neutronics calculations may be made using properly validated cornputational methods. C.2 If necessary, one or more parameters may be controlled either by additional measurements or screeniny, or perhaps by administrative procedures** to increase the critically safe operational limits. If all else fails, it may

"Critical parameter data for metal-water mixtures, both reflected and uureflected, appear in the literature. Specific references are listed in the bibliography for this appendix. The fictitious homogeneous metal-water mixture is usually given as $\alpha$ reference composition beccuse its critical mass is minimum at a given $\mathrm{H} /{ }^{235} \mathrm{U}$ or $\mathrm{H} /{ }^{239} \mathrm{Pu}$ ratio.

* Administrative control (e.g., on moisture content) is perhaps the most questionable or at least uncomfortable technique because people do make mistakes. 
be necessary to repackage material into smaller contciners for which critically safe limits can bo established.

Regardless of how the critically safe limits and operating procedures are established for a given assay system, they must be determined with the close cooperation of the Criticality Safety Officer at the plant where the equipment is being used. This person is an expert because of his experience and training, and ultimately, the criticality safety of operations involving handling, storage, and measurement of fissionable material is his responsibility. Do not bypass him.

There is considerable literature available concerning criticality safety and critical limits. Some of this literature is described here. An excellent and very readable report entitled "Criticality Control in Operations with Fissile Material "C.3 discusses in detail the factors influencing critical parameters, critical limil catc, computátional techniques, and general criticality-control practices. Several guides specify safety limits for a variety of conditions: two of these are "The Nuclear Safety Guide"C.A and "Nuclear Criticality Safety in Operations with Fissionable Materials Outside Reactors."C.5 Two of the several available compilations of experimental and/or calculated critical data are "Critical Dimensions of Systems Containing ${ }^{235} \mathrm{U},{ }^{239} \mathrm{Pu}$, and ${ }^{233} \mathrm{~J}^{\prime C} \mathrm{C} .6$ and "Criticality Data and Factors Affecting Criticality of Single Homogeneous Units."C.7

\section{REFERENCES}

C.1. American National Standard, "Safety in Conducting Subcritical Neutron-Multiplication Mearurement In Situ." Report No. ANSI N16.31969.
C.2. Hugh K. Clark, "Establishing Subcritical Limits," Savannah Hiver Laboratory report DP-MS73.27 (1973). This paper was presented at the Nuclear Criticality Safety Short Course at Taos, NM, May 6-11 (1973). It presents and discusses the draft of a proposed standard prepared by Work Group ANS 8.11 of the ANS Standards Committee for validating calculational methods of establishing subcritical limits for operations with tissionable materials.

C.3. H. C. Paxton, "Criticality Control in Operations with Fissile Material," Los Alamos Scientific Laboratory report LA-3377 (Rev.) (1972).

C.4. Nuclear Safety Guide, Subcommittee 8 of the ASA Sectional Committee N6, and Project 8 of the ANS Standards Committee, USAEC report TID. 7016, Rev. 1 (1961).

C.5. American National Standard, "Nuclear Criticality Safety in Operations with Fissionable Materials Ouiside Reactors." Report No. ANSI N16.1-1969 (Revision of N6.1-1964).

C.6. H. C. Paxton, J. T. Thomas, D. Callihan, and E. B. Johnson, "Critical Dimensions of Systems Containing ${ }^{230} \mathrm{U},{ }^{239} \mathrm{Pu}$, and ${ }^{23} 3^{3} \mathrm{U}, "$ USAEC report TID. 7028 (1964).

C.7. W. R. Stratton, "Criticality Data and Factors Affecting Criticality of Single Homogeneous Units," Los Alamos Scientific Laboratory report LA-3612 (1967). 